\title{
RESULTS OF OBSERVATIONS OF THE SUN, MOON, AND PLANETS.
}


RESULTS OF OBSERVATIONS OF THE SUN, MOON, AND PLANETS.

[For explanation, see page A Cuxvr.]

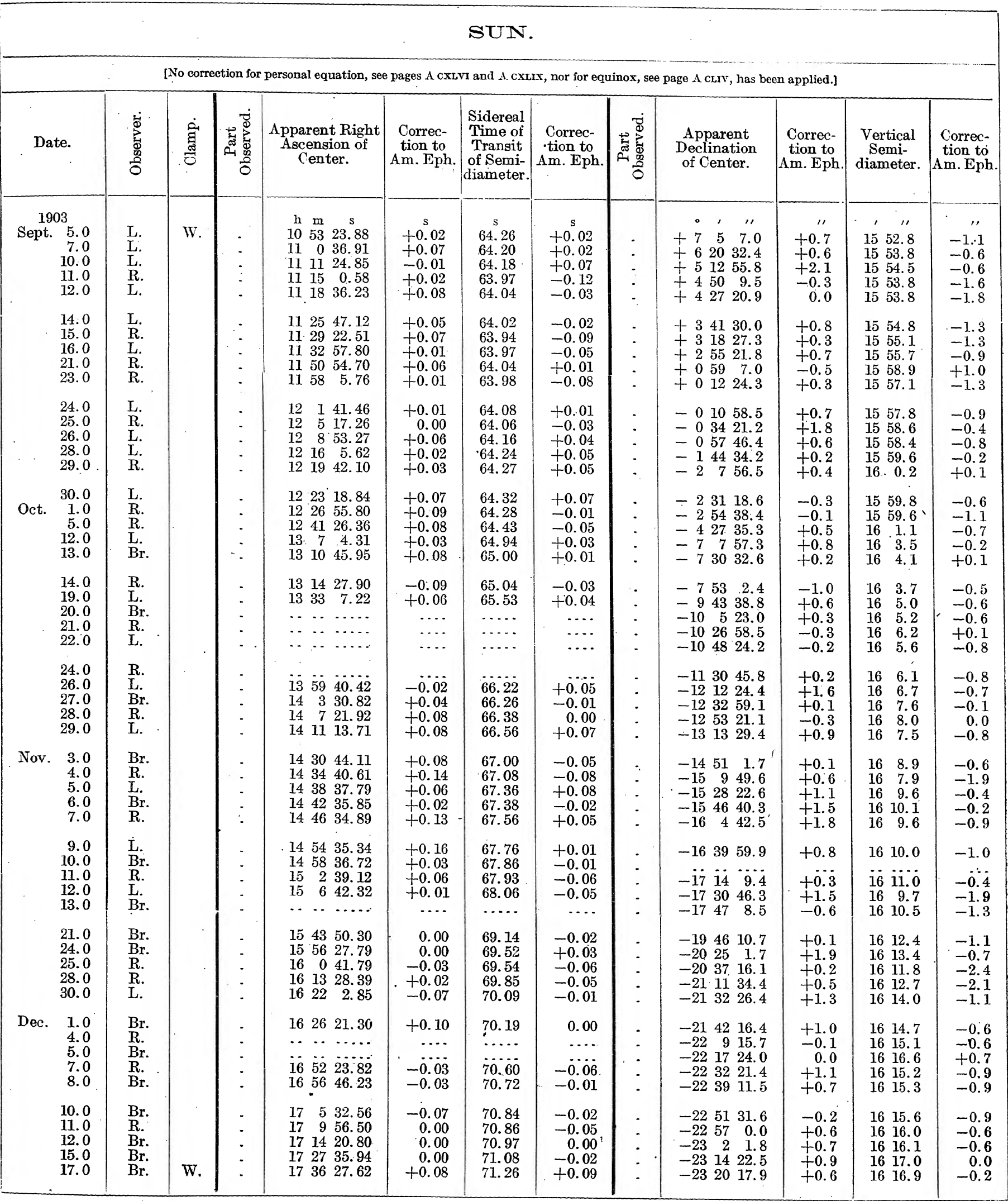


SUN.

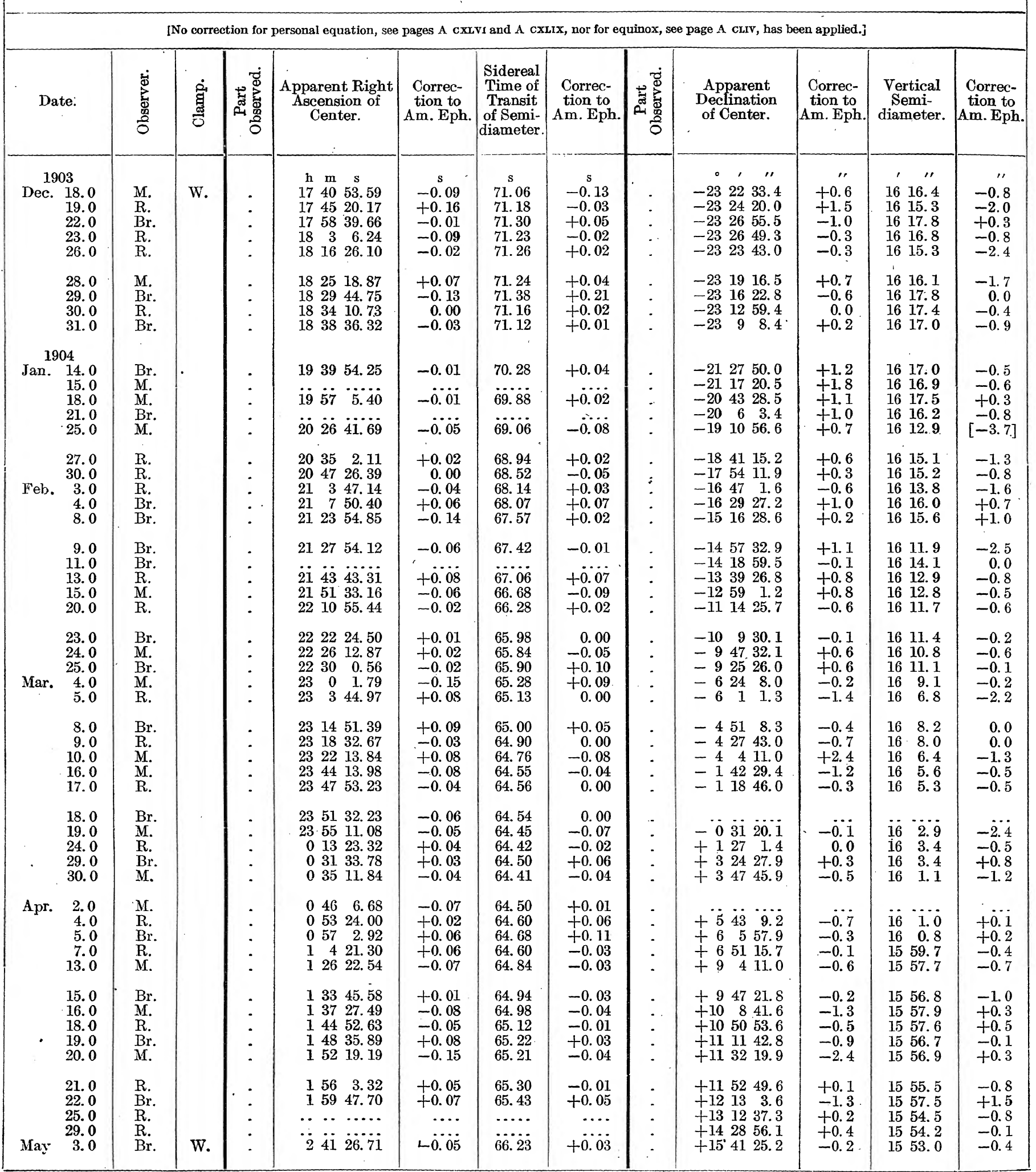


RESULTS OF OBSERVATIONS OF THE SUN, MOON, AND PLANETS.

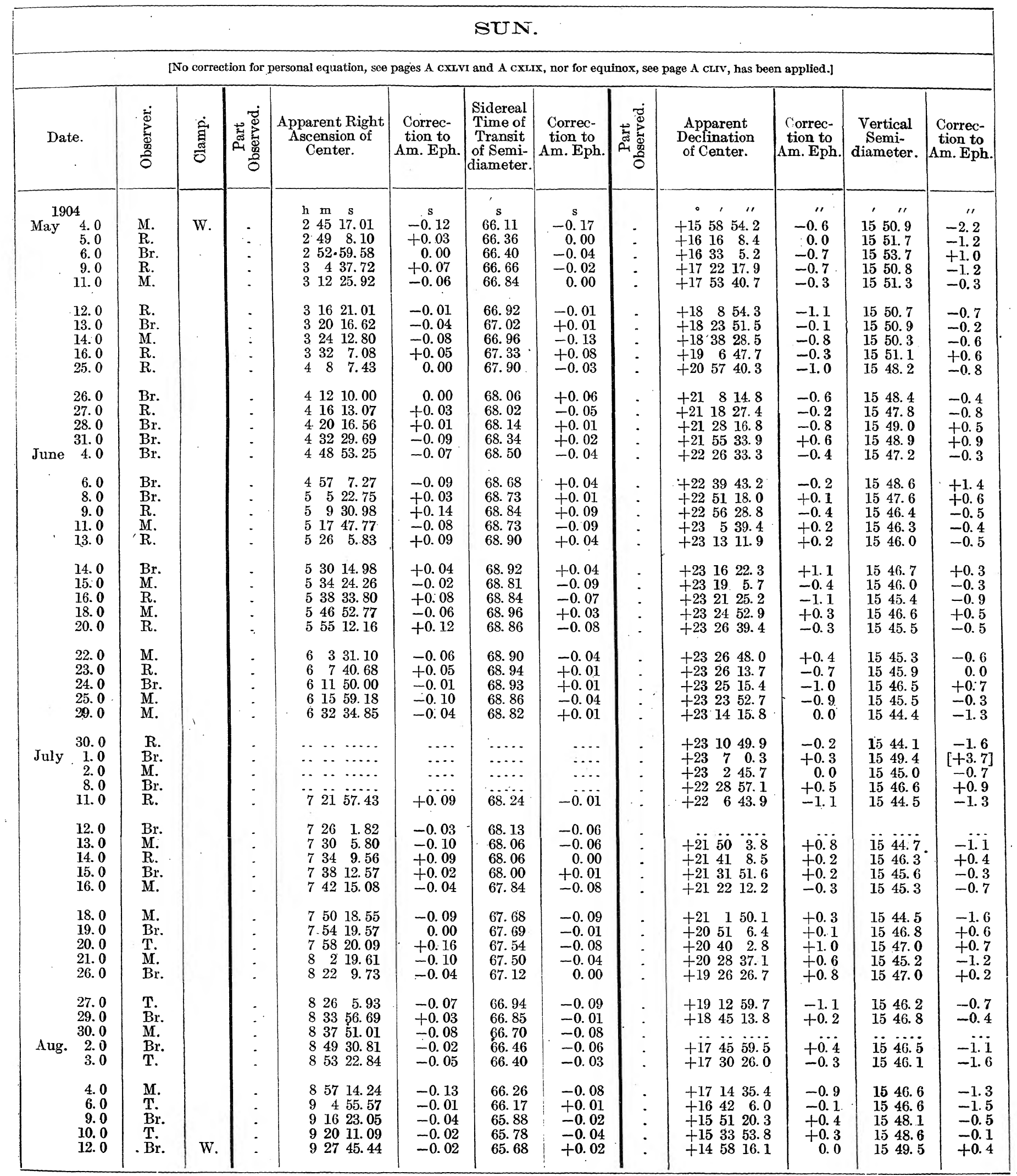




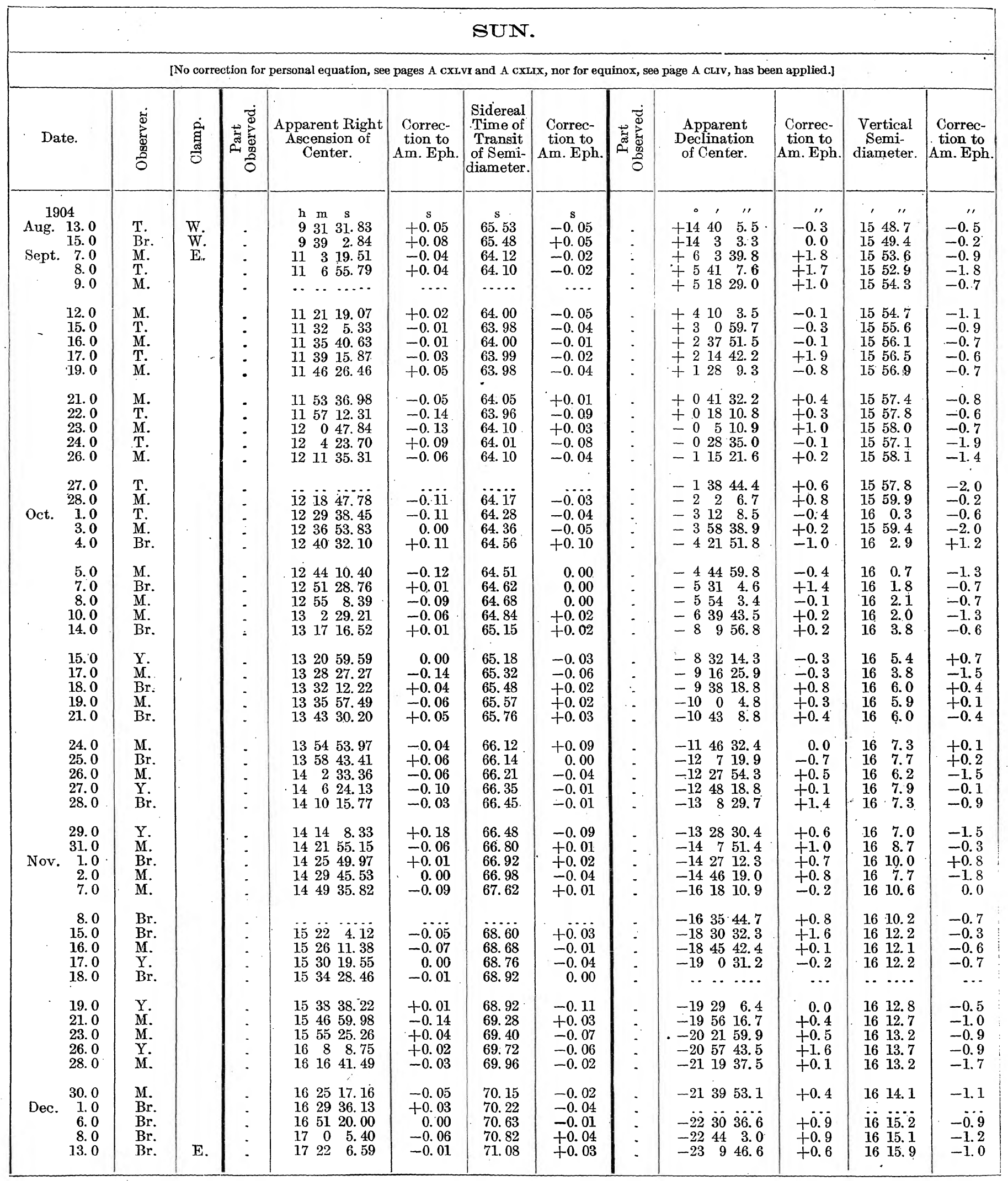




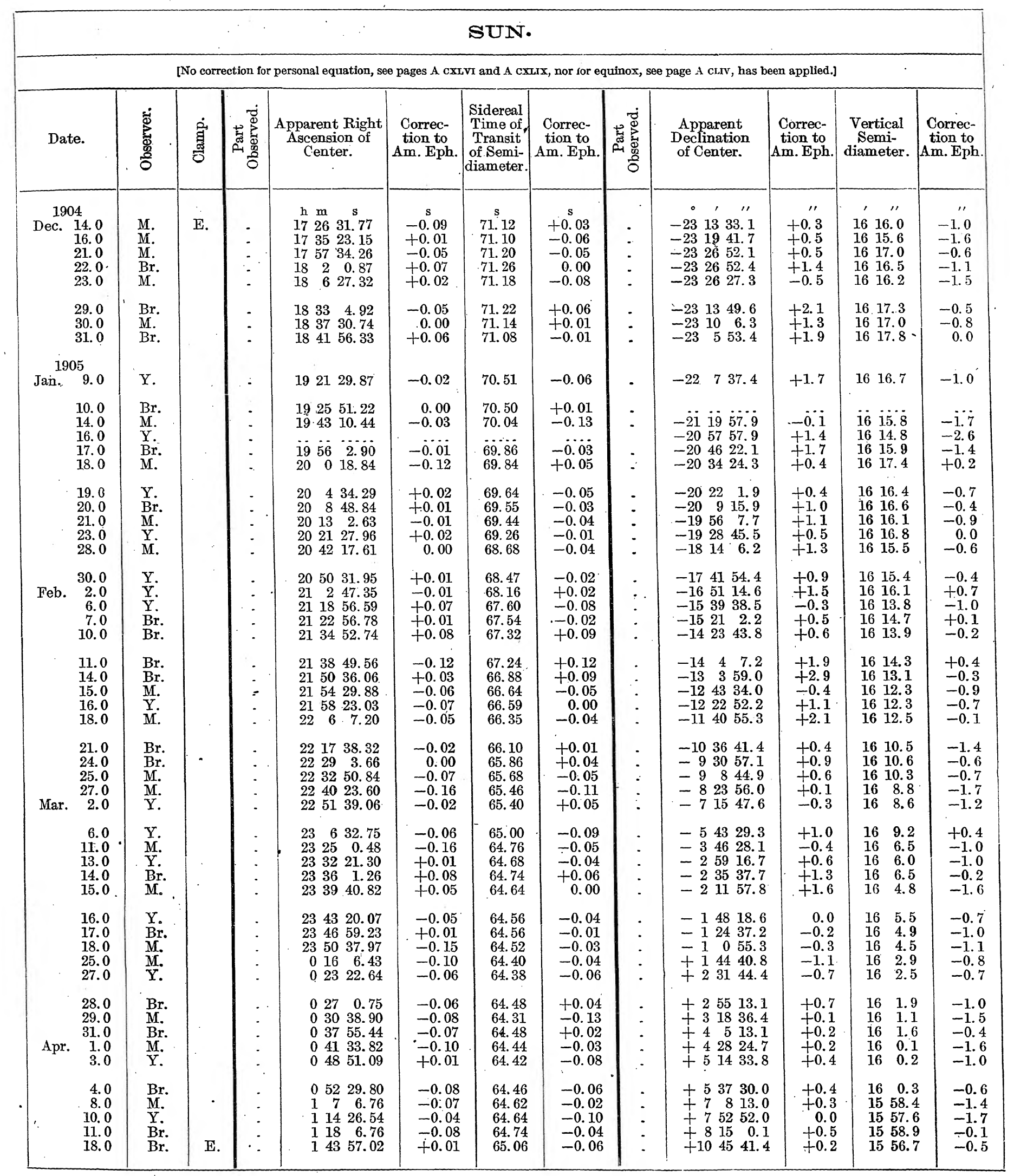




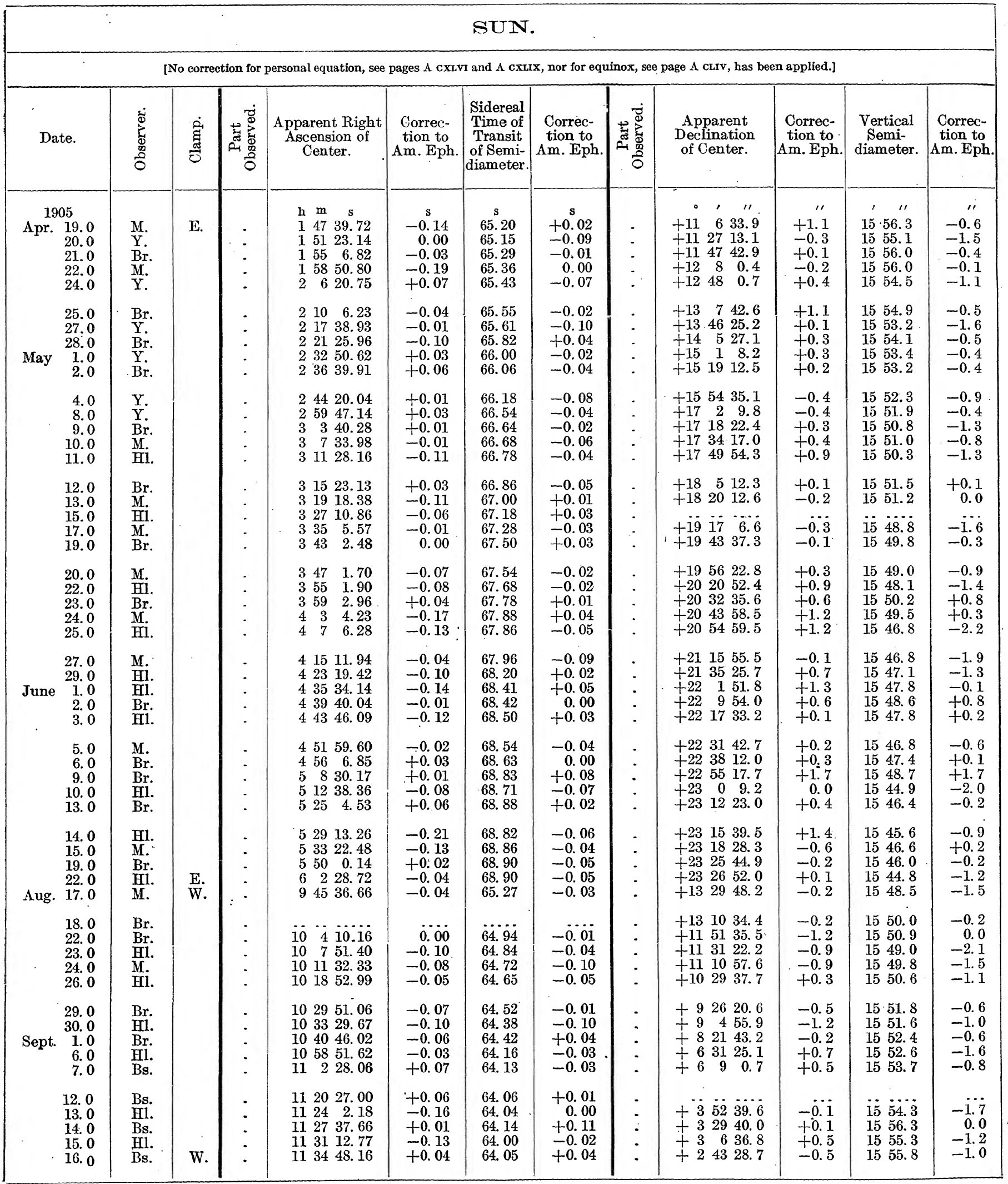




\begin{tabular}{|c|c|c|c|c|c|c|c|c|c|c|c|c|}
\hline \multicolumn{13}{|c|}{ SUN. } \\
\hline \multicolumn{13}{|c|}{ [No correction for personal equation, see pages A cxuvi and A cxurx, nor for equinox, see page A curv, has been applied.] } \\
\hline Date. & 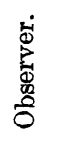 & 宊 & 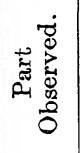 & $\begin{array}{l}\text { Apparent Right } \\
\text { Ascension of } \\
\text { Center. }\end{array}$ & $\begin{array}{c}\text { Correc- } \\
\text { tion to } \\
\text { Am. Eph. } \\
.\end{array}$ & $\begin{array}{c}\text { Sidereal } \\
\text { Time of } \\
\text { Transit } \\
\text { of Semi- } \\
\text { diameter. }\end{array}$ & $\begin{array}{c}\text { Correc- } \\
\text { tion to } \\
\text { Am. Eph. }\end{array}$ & 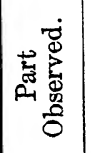 & $\begin{array}{l}\text { Apparent } \\
\text { Declination } \\
\text { of Center. }\end{array}$ & $\begin{array}{c}\text { Correc- } \\
\text { tion to } \\
\text { Am. Eph. }\end{array}$ & $\begin{array}{c}\text { Vertical } \\
\text { Semi- } \\
\text { diameter. }\end{array}$ & $\begin{array}{l}\text { Correc- } \\
\text { tion to } \\
\text { Am. Eph. }\end{array}$ \\
\hline $\begin{array}{c}1905 \\
\text { Sept. } 19.0 \\
20.0 \\
-21.0 \\
22.0 \\
23.0\end{array}$ & $\begin{array}{l}\text { Bs. } \\
\text { Hl. } \\
\text { Bs. } \\
\text { Hl. } \\
\text { Bs. }\end{array}$ & W. & : & \begin{tabular}{ccc}
$\mathrm{h}$ & $\mathrm{m}$ & \multicolumn{1}{c}{$\mathrm{s}$} \\
$\cdots$ & $\cdots$ & $\cdots$ \\
$\cdots$ & $\cdots$ & $\cdots$ \\
$\cdots$ & $\cdots$ & $\cdots$ \\
$\cdots$ & $\cdots$ & $\cdots$ \\
$\cdots$ & $\cdots$ & $\cdots$ \\
\end{tabular} & $\begin{array}{l}\mathrm{s} \\
\cdots \\
\cdots \\
\cdots \\
\cdots \\
\cdots\end{array}$ & $\begin{array}{l}\text { s } \\
\cdots \cdots \\
\cdots \cdots \\
\cdots \cdots \\
\cdots \cdots\end{array}$ & $\begin{array}{l}\mathrm{s} \\
\cdots \\
\cdots \\
\cdots \\
\cdots \\
\cdots\end{array}$ & : & 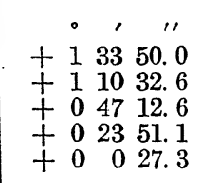 & $\begin{array}{r} \\
0.0 \\
+0.5 \\
+0.3 \\
+0.2 \\
-1.1\end{array}$ & $\begin{array}{cc}\prime & \prime \prime \\
15 & 55.9 \\
15 & 56.0 \\
15 & 58.1 \\
15 & 56.6 \\
15 & 58.4\end{array}$ & $\begin{array}{r}1 . \\
-1.7 \\
-1.8 \\
0.0 \\
-1.7 \\
-0.2\end{array}$ \\
\hline $\begin{array}{l}25.0 \\
26.0 \\
27.0 \\
28.0 \\
29.0\end{array}$ & $\begin{array}{l}\text { Bs. } \\
\text { Hl. } \\
\text { Bs. } \\
\text { Hl. } \\
\text { Bs. }\end{array}$ & & $\begin{array}{l}. \\
: \\
-\end{array}$ & $\begin{array}{rrr}12 & 7 & 7.25 \\
\dddot{12} & 10 & 139.59 \\
12 & 17 & 55.99 \\
12 & 21 & 32.83\end{array}$ & $\begin{array}{l}+0.01 \\
+00.01 \\
+0.06 \\
+0.08\end{array}$ & \begin{tabular}{l}
64.12 \\
\hdashline 3.18 \\
64.26 \\
64.20
\end{tabular} & $\begin{array}{l}+0.01 \\
+0.01 \\
+0.06 \\
-0.03\end{array}$ & : & $\begin{array}{rrrr}- & 0 & 46 & 19.4 \\
= & 1 & 9 & 44.4 \\
= & 1 & 33 & 7.7 \\
= & 1 & 56 & 31.6 \\
-2 & 19 & 53.2\end{array}$ & $\begin{array}{l}-0.2 \\
-0.9 \\
-0.2 \\
-0.6 \\
+0.5\end{array}$ & $\begin{array}{ll}15 & 58.2 \\
15 & 59.0 \\
15 & 59.9 \\
15 & 58.5 \\
15 & 59.5\end{array}$ & $\begin{array}{l}-0.9 \\
-0.4 \\
+0.2 \\
-1.5 \\
-0.7\end{array}$ \\
\hline 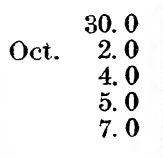 & $\begin{array}{l}\text { Hl. } \\
\text { Hl. } \\
\text { Bs. } \\
\text { Hl. } \\
\text { Bs. }\end{array}$ & & $:$ & $\begin{array}{ccc}12 & 25 & 9.58 \\
\dddot{12} & 39 & 30.03 \\
12 & 43 & 18.53 \\
12 & 50 & 36.37\end{array}$ & $\begin{array}{l}-0.12 \\
+001 \\
-0.07 \\
-0.08\end{array}$ & $\begin{array}{l}64.22 \\
64.50 \\
64.50 \\
64.60\end{array}$ & $\begin{array}{r}-0.05 \\
+0.05 \\
0.00 \\
-0.01\end{array}$ & $:$ & $\begin{array}{l}-24314.9 \\
=32952.0 \\
=41620.3 \\
=43929.6 \\
-52536.8\end{array}$ & $\begin{array}{r}+0.1 \\
+0.5 \\
-0.1 \\
0.0 \\
+0.6\end{array}$ & $\begin{array}{lr}15 & 59.0 \\
15 & 59.2 \\
16 & 1.3 \\
16 & 0.4 \\
16 & .1 .8\end{array}$ & $\begin{array}{l}-1.5 \\
-1.9 \\
-0.3 \\
-1.5 \\
-0.7\end{array}$ \\
\hline $\begin{array}{r}9.0 \\
10.0 \\
12.0 \\
13.0 \\
14.0\end{array}$ & $\begin{array}{l}\text { Hl. } \\
\text { Bs. } \\
\text { Bs. } \\
\text { Br. } \\
\text { Bs. }\end{array}$ & & : & $\begin{array}{rrr}12 & 57 & 55.80 \\
13 & 1 & 36.23 \\
13 & 8 & 58.39 \\
13 & 16 & 22.30\end{array}$ & $\begin{array}{r}-0.06 \\
+0.02 \\
+0.09 \\
-0.08\end{array}$ & $\begin{array}{l}64.76 \\
64.73 \\
65.00 \\
\not \dddot{65.12}\end{array}$ & $\begin{array}{l}+0.02 \\
-0.08 \\
+0.04 \\
+0.01\end{array}$ & : & 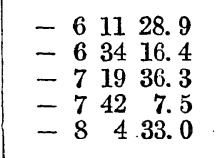 & $\begin{array}{r}-0.7 \\
0.0 \\
+0.3 \\
+0.3 \\
-0.3\end{array}$ & $\begin{array}{ll}16 & 1.8 \\
16 & 1.4 \\
16 & 3.1 \\
16 & 2.9 \\
16 & 3.9\end{array}$ & $\begin{array}{l}-1.2 \\
-1.9 \\
-0.8 \\
-1.3 \\
-0.5\end{array}$ \\
\hline $\begin{array}{l}16.0 \\
17.0 \\
27.0 \\
28.0 \\
31.0\end{array}$ & $\begin{array}{l}\mathrm{Hl} . \\
\mathrm{Br} . \\
\mathrm{Hl} . \\
\text { Bs. } \\
\mathrm{Br} .\end{array}$ & & $:$ & $\begin{array}{rrr}13 & 27 & 32.64 \\
14 & 5 & 28.71 \\
14 & 9 & 20.30 \\
14 & 20 & 59.60\end{array}$ & $\begin{array}{l}-0.01 \\
-0.09 \\
-0.04 \\
+0.07\end{array}$ & $\begin{array}{l}65.30 \\
66.31 \\
66.40 \\
66.80\end{array}$ & $\begin{array}{l}-0.05 \\
-0.02 \\
-0.03 \\
+0.04\end{array}$ & $\dot{5}$ & $\begin{array}{rrrr}-8 & 49 & 1.7 \\
-\dddot{12} & \ddot{43} & 20.2 \\
-13 & 3 & 41.9 \\
-14 & 3 & 13.5\end{array}$ & $\begin{array}{l}+0.2 \\
+0.8 \\
+0.1 \\
+0.8\end{array}$ & $\begin{array}{rr}16 & 2.8 \\
\dddot{16} & \dddot{6} .0 \\
16 & 7.3 \\
16 & 7.8\end{array}$ & $\begin{array}{r}-2.2 \\
-1.9 \\
-0.8 \\
-1.1\end{array}$ \\
\hline Nov. $\begin{array}{l}1.0 \\
2.0 \\
4.0 \\
7.0 \\
9.0\end{array}$ & $\begin{array}{l}\text { Bs. } \\
\text { Hl. } \\
\text { Bs. } \\
\text { Hl. } \\
\text { Hl. }\end{array}$ & & : & $\begin{array}{lll}14 & 24 & 54.15 \\
14 & 28 & 49.43 \\
14 & 36 & 42.75 \\
14 & 48 & 38.37 \\
14 & 56 & 39.67\end{array}$ & $\begin{array}{l}+0.02 \\
-0.09 \\
+0.09 \\
-0.06 \\
-0.06\end{array}$ & $\begin{array}{l}66.83 \\
67.11 \\
67.24 \\
67.61 \\
67.92\end{array}$ & $\begin{array}{l}-0.05 \\
+0.12 \\
+0.01 \\
+0.02 \\
+0.10\end{array}$ & $\begin{array}{l}- \\
\vdots \\
\vdots\end{array}$ & $\begin{array}{lll}-14 & 22 & 38.1 \\
-14 & 41 & 49.2 \\
-15 & 19 & 25.3 \\
-16 & 13 & 56.7 \\
-16 & 48 & 55.7\end{array}$ & $\begin{array}{l}+0.5 \\
-0.2 \\
+1.1 \\
+0.7 \\
+1.2\end{array}$ & $\begin{array}{ll}16 & 8.8 \\
16 & 9.0 \\
16 & 9.6 \\
16 & 8.1 \\
16 & 9.8\end{array}$ & $\begin{array}{l}-0.4 \\
-0.4 \\
-0.3 \\
-2.5 \\
-1.3\end{array}$ \\
\hline $\begin{array}{l}10.0 \\
11.0 \\
14.0 \\
15.0 \\
17.0\end{array}$ & $\begin{array}{l}\text { Bs. } \\
\text { Hl. } \\
\text { Br. } \\
\text { Bs. } \\
\text { Br. }\end{array}$ & & : & $\begin{array}{rrr}15 & 0 & 41.59 \\
15 & 4 & 44.34 \\
15 & 16 & 57.73 \\
15 & 21 & 4.04 \\
15 & 29 & 18.85\end{array}$ & $\begin{array}{l}-0.06 \\
-0.07 \\
-0.04 \\
+0.09 \\
-0.05\end{array}$ & $\begin{array}{l}67.90 \\
68.17 \\
68.49 \\
68.48 \\
68.78\end{array}$ & $\begin{array}{l}-0.04 \\
+0.11 \\
+0.07 \\
-0.06 \\
+0.01\end{array}$ & $:$ & $\begin{array}{rrr}-17 & 6 & 0.3 \\
-17 & 22 & 46.7 \\
-18 & 11 & 19.0 \\
-18 & 26 & 49.4 \\
-18 & 56 & 57.3\end{array}$ & $\begin{array}{l}+0.7 \\
+1.0 \\
-0.6 \\
+1.4 \\
-0.3\end{array}$ & $\begin{array}{ll}16 & 11.5 \\
16 & 10.7 \\
16 & 13.0 \\
16 & 10.1 \\
16 & 12.8\end{array}$ & $\begin{array}{r}+0.1 \\
-0.9 \\
+0.8 \\
-2.3 \\
0.0\end{array}$ \\
\hline $\begin{array}{r}21.0 \\
23.0 \\
24.0 \\
27.0 \\
\text { Dec. } \quad 1.0\end{array}$ & $\begin{array}{l}\text { Br. } \\
\text { Hl. } \\
\text { Br. } \\
\text { Hl. } \\
\text { Br. }\end{array}$ & & : & $\begin{array}{lll}15 & 45 & 58.83 \\
15 & 54 & 23.92 \\
15 & 58 & 37.68 \\
16 & 11 & 23.36 \\
16 & 28 & 34.52\end{array}$ & $\begin{array}{r}-0.12 \\
-0.05 \\
0.00 \\
-0.10 \\
+0.02\end{array}$ & $\begin{array}{l}69.24 \\
69.52 \\
69.50 \\
69.94 \\
70.33\end{array}$ & $\begin{array}{l}+0.02 \\
+0.08 \\
-0.04 \\
+0.08 \\
+0.09\end{array}$ & $:$ & $\begin{array}{rrr}-19 & 53 & 1.7 \\
-20 & 18 & 58.8 \\
-20 & 31 & 21.3 \\
-21 & 6 & 15.6 \\
-21 & 47 & 10.4\end{array}$ & $\begin{array}{l}+2.0 \\
-0.5 \\
+0.9 \\
+0.1 \\
+1.5\end{array}$ & $\begin{array}{ll}16 & 14.3 \\
16 & 13.8 \\
16 & 13.1 \\
16 & 13.5 \\
16 & 14.7\end{array}$ & $\begin{array}{l}+0.7 \\
-0.2 \\
-1.1 \\
-1.2 \\
-0.6\end{array}$ \\
\hline $\begin{array}{r}4.0 \\
5.0 \\
7.0 \\
8.0 \\
11.0\end{array}$ & $\begin{array}{l}\mathrm{Hl} . \\
\mathrm{Br} . \\
\mathrm{Hl} . \\
\mathrm{Br} . \\
\mathrm{Hl} .\end{array}$ & & $\therefore$ & $\begin{array}{rrr}16 & 41 & 34.47 \\
16 & 45 & 55.52 \\
16 & 54 & 39.39 \\
16 & 59 & 2.12 \\
17 & 12 & 12.85\end{array}$ & $\begin{array}{l}+0.01 \\
-0.08 \\
-0.09 \\
-0.03 \\
-0.04\end{array}$ & $\begin{array}{l}70.53 \\
70.58 \\
70.80 \\
70.75 \\
71.06\end{array}$ & $\begin{array}{l}+0.05 \\
+0.02 \\
+0.10 \\
-0.02 \\
+0.11\end{array}$ & $:$ & $\begin{array}{lll}-22 & 13 & 31.9 \\
-22 & 21 & 27.2 \\
-22 & 35 & 56.2 \\
-22 & 42 & 33.6 \\
-22 & 59 & 40.4\end{array}$ & $\begin{array}{l}-0.3 \\
-0.5 \\
+2.4 \\
+1.0 \\
+0.9\end{array}$ & $\begin{array}{ll}16 & 15.2 \\
16 & 15.7 \\
16 & 16.1 \\
16 & 15.1 \\
16 & 15.4\end{array}$ & $\begin{array}{l}-0.6 \\
-0.3 \\
-0.1 \\
-1.3 \\
-1.3\end{array}$ \\
\hline $\begin{array}{l}12.0 \\
13.0 \\
18.0 \\
22.0 \\
26.0\end{array}$ & $\begin{array}{l}\text { Br. } \\
\text { Bs. } \\
\text { Bs. } \\
\text { Bs. } \\
\text { Hl. }\end{array}$ & & : & $\begin{array}{rrr}17 & 16 & 37.28 \\
17 & 21 & 2.09 \\
17 & 43 & 10.14 \\
18 & 0 & 56.08 \\
18 & 18 & 42.51\end{array}$ & $\begin{array}{r}0.00 \\
+0.06 \\
-0.02 \\
-0.03 \\
-0.18\end{array}$ & $\begin{array}{l}71.08 \\
71.04 \\
71.08 \\
71.16 \\
71.22\end{array}$ & $\begin{array}{l}+0.08 \\
-0.01 \\
-0.12 \\
-0.10 \\
-0.02\end{array}$ & $:$ & $\begin{array}{rrr}-23 & 4 & 28.4 \\
-23 & 8 & 48.5 \\
-23 & 23 & 33.2 \\
-23 & 26 & 54.0 \\
-23 & 22 & 44.1\end{array}$ & $\begin{array}{l}+0.7 \\
+0.9 \\
+0.5 \\
+1.9 \\
+1.8\end{array}$ & $\begin{array}{ll}16 & 16.6 \\
16 & 15.8 \\
16 & 16.6 \\
16 & 16.2 \\
16 & 16.0\end{array}$ & $\begin{array}{l}-0.2 \\
-1.1 \\
-0.7 \\
-1.3 \\
-1.7\end{array}$ \\
\hline $\begin{array}{l}28.0 \\
29.0 \\
30.0\end{array}$ & $\begin{array}{l}\mathrm{Hl} . \\
\mathrm{Br} . \\
\mathrm{Hl} .\end{array}$ & & . & $\begin{array}{rrr}18 & 27 & 35.47 \\
18 & 32 & 1.56 \\
18 & 36 & 27.33\end{array}$ & $\begin{array}{l}+0.03 \\
+0.02 \\
-0.08\end{array}$ & $\begin{array}{l}71.14 \\
71.20 \\
71.24\end{array}$ & $\begin{array}{l}-0.05 \\
+0.04 \\
+0.11\end{array}$ & $:$ & $\begin{array}{llr}-23 & 17 & 51.4 \\
-23 & 14 & 41.1 \\
-23 & 11 & 4.2\end{array}$ & $\begin{array}{r}0.0 \\
+1.0 \\
+0.5\end{array}$ & $\begin{array}{ll}16 & 15.7 \\
16 & 16.8 \\
16 & 17.7\end{array}$ & $\begin{array}{l}-2.1 \\
-1.0 \\
-0.1\end{array}$ \\
\hline Jan. $\quad 2.0$ & Br. & W. & • & 184943.35 & +0.06 & 70.96 & -0.06 & $\cdot$ & $-22 \quad 5724.6$ & +1.3 & 1616.8 & -1.1 \\
\hline
\end{tabular}




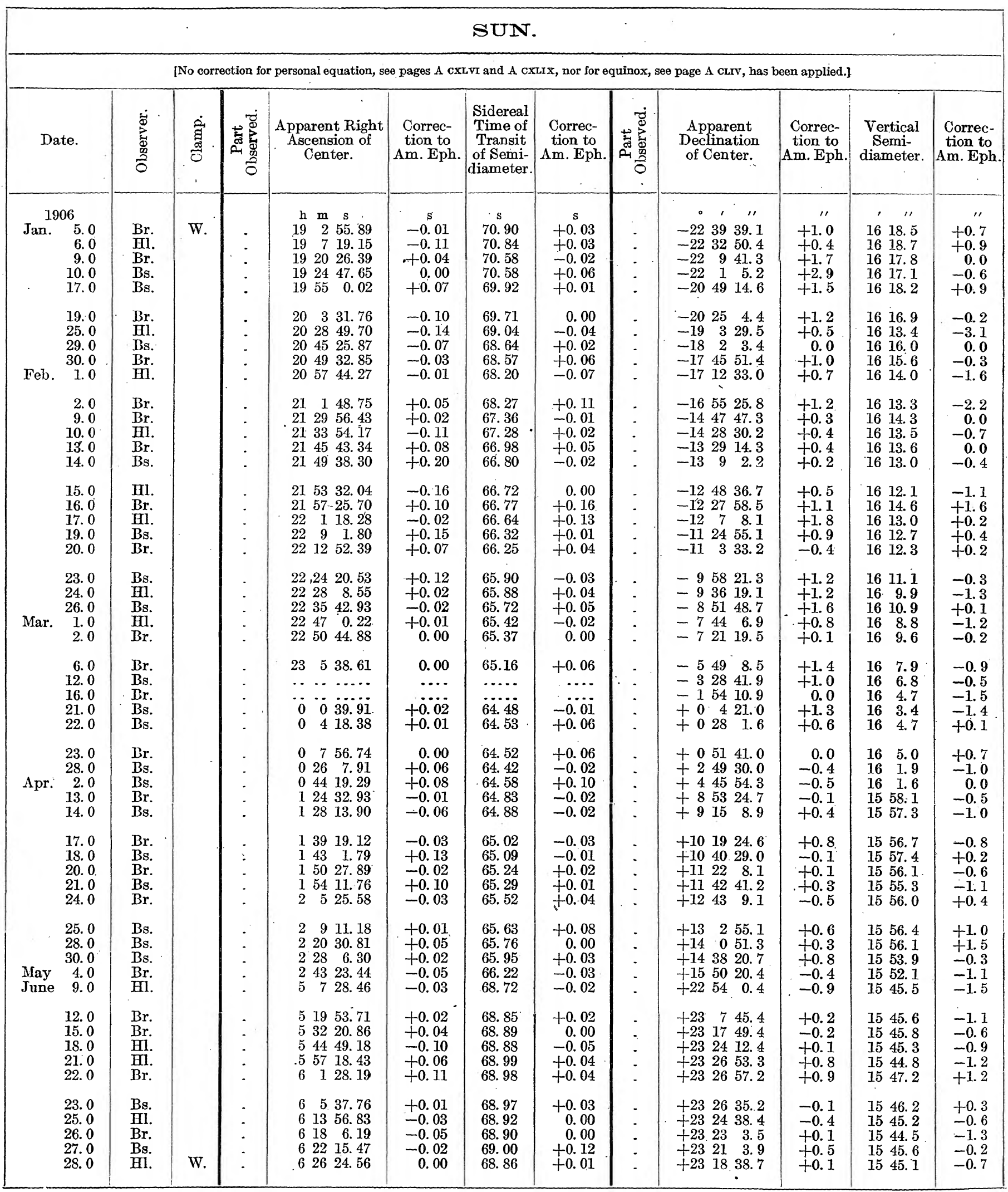


RESULTS OF OBS ERVATIONS OF THE SUN, MOON, AND PLANETS.

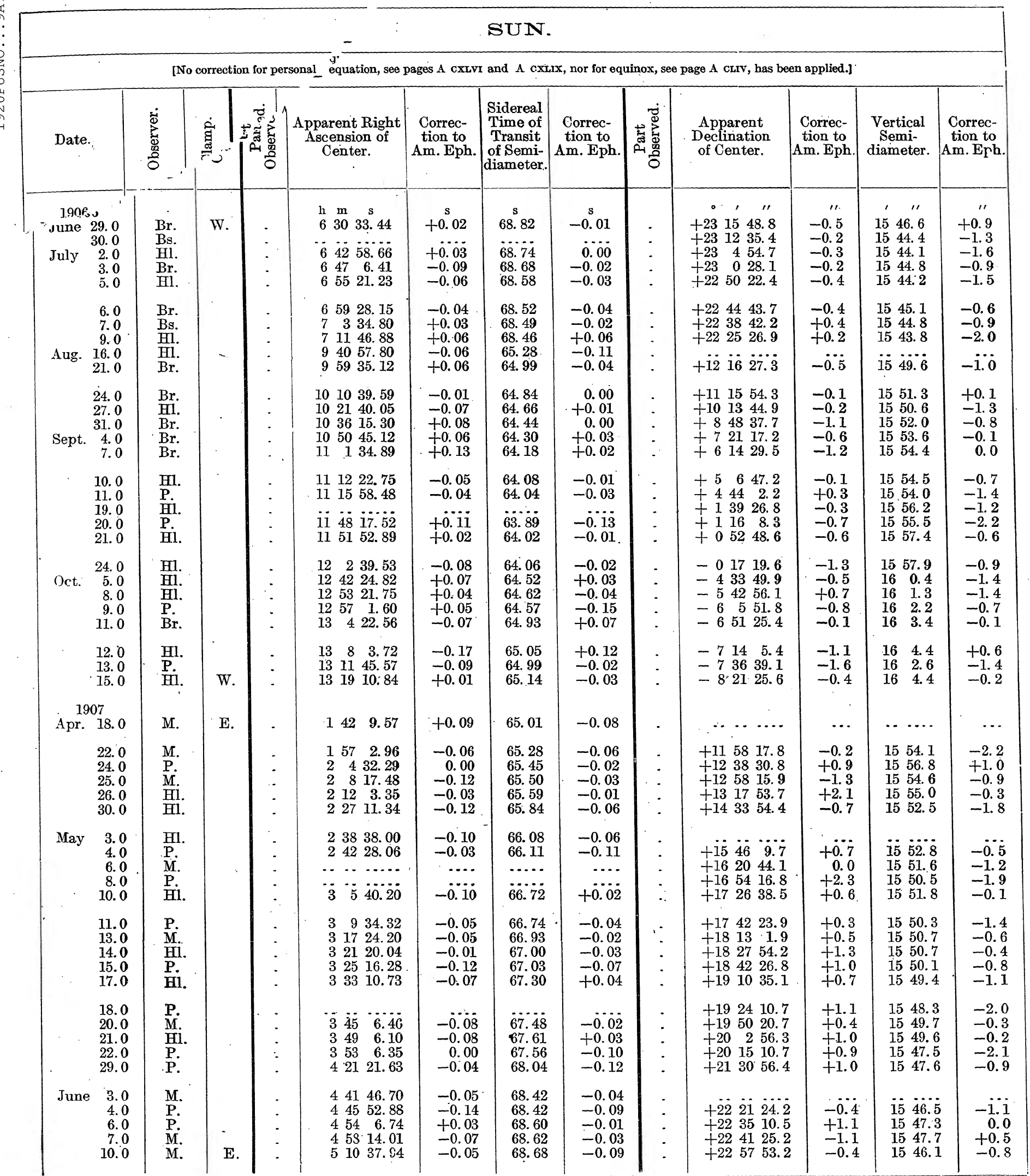




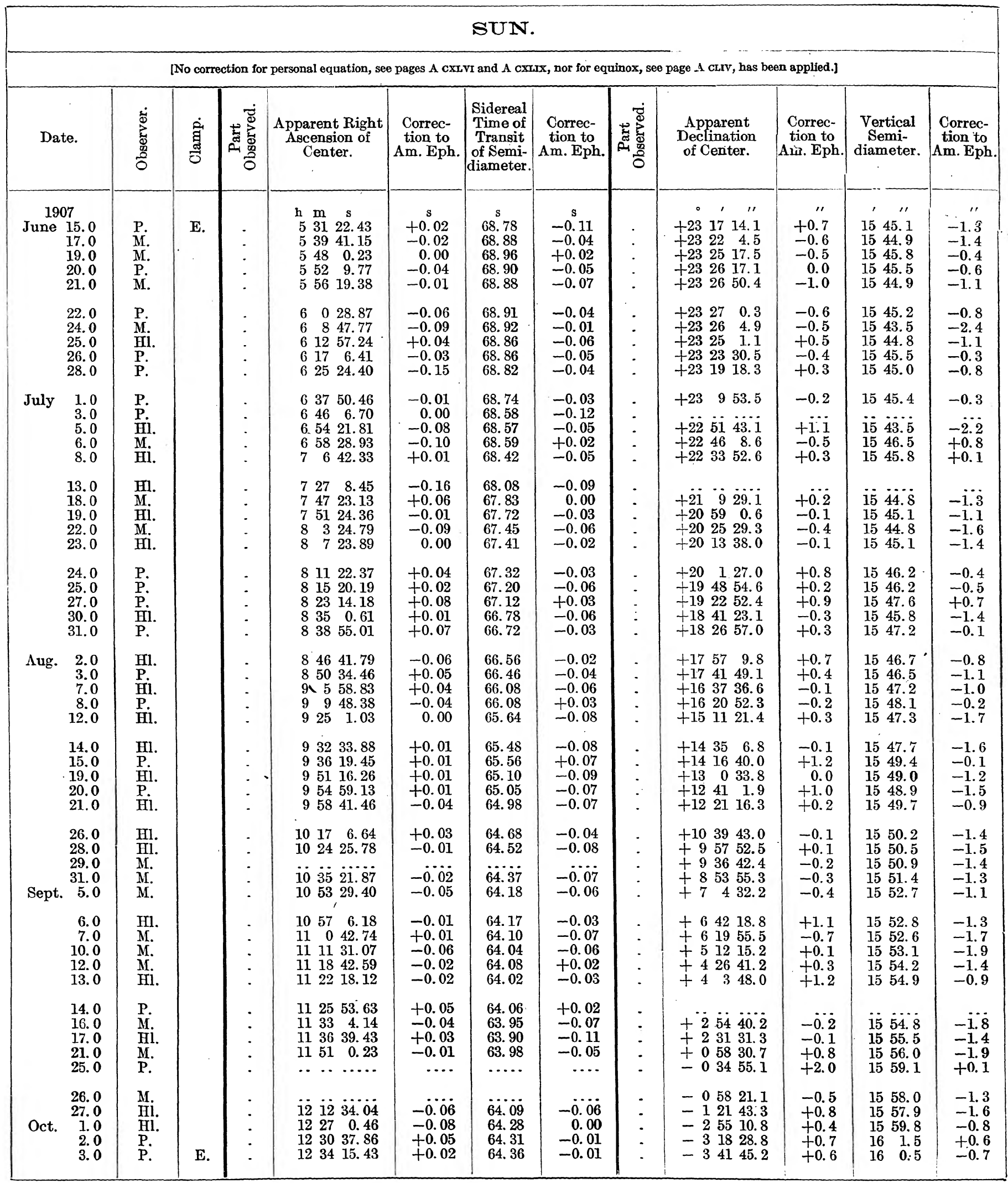




\begin{tabular}{|c|c|c|c|c|c|c|c|c|c|c|c|c|}
\hline \multicolumn{6}{|c|}{ · } & \multicolumn{7}{|l|}{ SUN. } \\
\hline \multicolumn{13}{|c|}{ [No correction for personal equation, see pages $\mathrm{A}$ Cxuvr and A cxux, nor for equinox, see page $\mathrm{A}$ curv, has been applied.] } \\
\hline Date. & $\begin{array}{l}\dot{\bar{\Phi}} \\
\stackrel{0}{0} \\
\text { on } \\
0\end{array}$ & 言 & 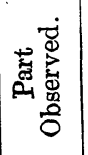 & $\begin{array}{l}\text { Apparent Right } \\
\text { Ascension of } \\
\text { Center. }\end{array}$ & $\begin{array}{c}\text { Correc- } \\
\text { tion to } \\
\text { Am. Eph. }\end{array}$ & $\begin{array}{c}\text { Sidereal } \\
\text { Time of } \\
\text { Transit } \\
\text { of Semi- } \\
\text { diameter. }\end{array}$ & $\begin{array}{c}\text { Correc- } \\
\text { tion to } \\
\text { Am. Eph. }\end{array}$ & 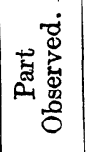 & $\begin{array}{l}\text { Apparent } \\
\text { Declination } \\
\text { of Center. }\end{array}$ & $\mid \begin{array}{c}\text { Correc- } \\
\text { tion to } \\
\text { Am. Eph. }\end{array}$ & $\begin{array}{c}\text { Vertical } \\
\text { Semi- } \\
\text { diameter. }\end{array}$ & $\begin{array}{l}\text { Correc- } \\
\text { tion to } \\
\text { Am. Eph. }\end{array}$ \\
\hline $\begin{array}{r}1907 \\
\text { Oct. } \quad 5.0 \\
7.0 \\
8.0 \\
9.0 \\
10.0\end{array}$ & $\begin{array}{l}\mathrm{P} . \\
\mathrm{M} . \\
\mathrm{P} . \\
\mathrm{M} .\end{array}$ & E. & $\dot{5}$ & \begin{tabular}{ccc}
$\mathrm{h}$ & $\mathrm{m}$ & \multicolumn{1}{c}{$\mathrm{s}$} \\
12 & 41 & 31.77 \\
12 & 48 & 49.18 \\
12 & 52 & 28.68 \\
12 & 56 & 8.46 \\
12 & 59 & 48.84
\end{tabular} & $\begin{array}{c}\mathrm{s} \\
+0.17 \\
-0.05 \\
+0.04 \\
0.00 \\
+0.14\end{array}$ & $\begin{array}{l}\mathrm{s} \\
64.46 \\
64.54 \\
64.68 \\
64.66 \\
64.74\end{array}$ & $\begin{array}{c}\mathrm{s} \\
-0.01 \\
-0.04 \\
+0.04 \\
-0.04 \\
-0.03\end{array}$ & $\dot{5}$ & 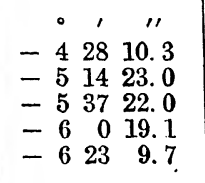 & $\begin{array}{r}\quad 1 \\
+0.6 \\
+0.3 \\
+1.7 \\
+0.6 \\
+1.4\end{array}$ & $\begin{array}{cc} & \prime \prime \\
16 & 1.6 \\
16 & 0.8 \\
16 & 1.7 \\
16 & 1.3 \\
16 & 2.5\end{array}$ & $\begin{array}{l}\quad \prime \prime \\
-0.1 \\
-1.5 \\
=0.8 \\
=-1.5 \\
-0.6\end{array}$ \\
\hline $\begin{array}{l}11.0 \\
12.0 \\
15.0 \\
16.0 \\
17.0\end{array}$ & $\begin{array}{l}\mathrm{Mr} . \\
\mathrm{M} . \\
\mathrm{Hi} . \\
\mathrm{P} . \\
\mathrm{M} .\end{array}$ & & $\therefore$ & $\begin{array}{rrr}13 & 3 & 29.40 \\
13 & 7 & 10.56 \\
13 & 18 & 16.84 \\
13 & 22 & 0.08 \\
13 & 25 & 43.72\end{array}$ & $\begin{array}{l}+0.01 \\
+0.04 \\
-0.03 \\
+0.06 \\
0.00\end{array}$ & $\begin{array}{l}64.78 \\
64.86 \\
65.20 \\
65.26 \\
65.23\end{array}$ & $\begin{array}{l}-0.06 \\
-0.06 \\
+0.05 \\
+0.03 \\
-0.09\end{array}$ & $:$ & $\begin{array}{rrrr}-6 & 45 & 56.4 \\
-\because 8 & 16 & \dddot{1} .1 \\
-8 & 38 & 17.6 \\
-9 & 0 & 25.4\end{array}$ & $\begin{array}{l}+0.9 \\
+\dddot{1} .1 \\
+0.4 \\
-0.1\end{array}$ & $\begin{array}{rr}16 & 2.3 \\
\dddot{16} & \because 7.8 \\
16 & 3.9 \\
16 & 3.5\end{array}$ & $\begin{array}{r}-1.1 \\
-1.7 \\
-0.9 \\
-1.6\end{array}$ \\
\hline $\begin{array}{l}18.0 \\
19.0 \\
21.0 \\
22.0 \\
23.0\end{array}$ & $\begin{array}{l}\mathrm{Hl} . \\
\mathrm{P} . \\
\mathrm{M} . \\
\mathrm{H} . \\
\mathrm{P} .\end{array}$ & & $:$ & $\begin{array}{lll}13 & 29 & 28.02 \\
13 & 33 & 12.89 \\
13 & 40 & 44.43 \\
13 & 44 & 31.15 \\
13 & 48 & 18.70\end{array}$ & $\begin{array}{l}+0.03 \\
+0.05 \\
0.00 \\
-0.04 \\
+0.09\end{array}$ & $\begin{array}{l}65.34 \\
65.54 \\
65.60 \\
65.73 \\
65.88\end{array}$ & $\begin{array}{l}-0.06 \\
+0.05 \\
-0.07 \\
-0.03 \\
+0.02\end{array}$ & $:$ & 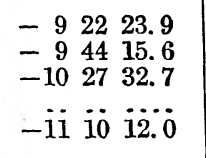 & $\begin{array}{l}+0.9 \\
+0.5 \\
+0.1 \\
+0.4\end{array}$ & $\begin{array}{rr}16 & 4.0 \\
16 & 7.0 \\
16 & 5.4 \\
16 & -0.8\end{array}$ & $\begin{array}{r}-1.3 \\
+1.4 \\
-0.8 \\
-0.9\end{array}$ \\
\hline $\begin{array}{l}24.0 \\
25.0 \\
26.0 \\
29.0 \\
30.0\end{array}$ & $\begin{array}{l}\text { M. } \\
\text { P. } \\
\text { Hil. } \\
\text { Hl. } \\
\text { P. }\end{array}$ & & : & $\begin{array}{rrr}13 & 52 & 6.64 \\
13 & 55 & 55.66 \\
13 & 59 & 45.17 \\
14 & 11 & 18.20 \\
14 & 15 & 10.92\end{array}$ & $\begin{array}{l}-0.09 \\
+0.09 \\
+0.05 \\
-0.05 \\
+0.08\end{array}$ & $\begin{array}{l}65.93 \\
66.10 \\
66.27 \\
66.50 \\
66.62\end{array}$ & $\begin{array}{l}-0.03 \\
+0.04 \\
+0.11 \\
+0.02 \\
+0.03\end{array}$ & $\therefore$ & $\begin{array}{lll}-11 & 31 & 17.0 \\
-11 & 52 & 11.7 \\
-12 & 12 & 55.1 \\
-13 & 13 & 57.6 \\
-13 & 33 & 54.5\end{array}$ & $\begin{array}{l}+0.3 \\
+0.2 \\
+0.6 \\
+0.9 \\
+0.8\end{array}$ & $\begin{array}{ll}16 & 6.7 \\
16 & 7.0 \\
16 & 7.4 \\
16 & 8.6 \\
16 & 6.1\end{array}$ & $\begin{array}{l}-0.3 \\
-0.2 \\
-0.1 \\
+0.4 \\
-2.4\end{array}$ \\
\hline 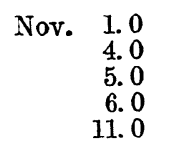 & $\begin{array}{l}\mathrm{Hl} . \\
\mathrm{M} . \\
\mathrm{Hi} \\
\mathrm{P} . \\
\mathrm{M} .\end{array}$ & & $:$ & $\begin{array}{rrr}14 & 22 & 58.37 \\
14 & 34 & 45.84 \\
14 & 38 & 43.38 \\
14 & 42 & 41.72 \\
15 & 2 & 45.51\end{array}$ & $\begin{array}{l}-0.05 \\
-0.01 \\
+0.07 \\
+0.14 \\
+0.04\end{array}$ & $\begin{array}{l}66.79 \\
67.10 \\
67.28 \\
67.41 \\
67.97\end{array}$ & $\begin{array}{r}-0.03 \\
-0.07 \\
-0.01 \\
0.00 \\
-0.03\end{array}$ & $:$ & $\begin{array}{rrr}-14 & 13 & 8.7 \\
-15 & 10 & 18.4 \\
-15 & 28 & 52.3 \\
-15 & 47 & 10.7 \\
-17 & 14 & 40.0\end{array}$ & $\begin{array}{l}+1.2 \\
+0.7 \\
+0.7 \\
+0.9 \\
+0.7\end{array}$ & $\begin{array}{lr}16 & 6.7 \\
16 & 8.5 \\
16 & 8.3 \\
16 & 10.1 \\
16 & 10.4\end{array}$ & $\begin{array}{l}-2.3 \\
-1.2 \\
-1.7 \\
-0.1 \\
-1.0\end{array}$ \\
\hline $\begin{array}{l}13.0 \\
14.0 \\
15.0 \\
16.0 \\
19.0\end{array}$ & $\begin{array}{l}\mathrm{P} . \\
\mathrm{M} . \\
\mathrm{Hi} . \\
\mathrm{P} . \\
\mathrm{Hil}\end{array}$ & & $:$ & $\begin{array}{rrr}15 & 10 & 52.92 \\
15 & 14 & 57.60 \\
15 & 19 & 3.62 \\
15 & 23 & 10.29 \\
15 & 35 & 34.82\end{array}$ & $\begin{array}{l}+0.09 \\
-0.16 \\
+0.08 \\
+0.14 \\
-0.11\end{array}$ & $\begin{array}{l}68.34 \\
68.34 \\
68.40 \\
68.59 \\
68.93\end{array}$ & $\begin{array}{l}+0.10 \\
-0.02 \\
-0.08 \\
-0.01 \\
-0.02\end{array}$ & $:$ & $\begin{array}{rrr}-17 & 47 & 37.3 \\
-18 & 3 & 38.6 \\
-18 & 19 & 20.3 \\
-18 & 34 & 42.9 \\
-19 & 18 & 51.5\end{array}$ & $\begin{array}{l}+1.2 \\
+1.1 \\
+1.4 \\
+1.5 \\
+0.9\end{array}$ & $\begin{array}{ll}16 & 10.9 \\
16 & 10.3 \\
16 & 10.7 \\
16 & 11.1 \\
16 & 12.3\end{array}$ & $\begin{array}{l}-1.0 \\
-1.8 \\
-1.6 \\
-1.4 \\
-0.9\end{array}$ \\
\hline $\begin{array}{r}25.0 \\
26.0 \\
27.0 \\
30.0 \\
\text { Dec. } 2.0\end{array}$ & $\begin{array}{l}\text { M. } \\
\text { P. } \\
\text { Hil. } \\
\text { P. }\end{array}$ & & $:$ & $\begin{array}{rrr}16 & 0 & 46.65 \\
16 & 5 & 1.48 \\
16 & 9 & 16.79 \\
16 & 22 & 8.00 \\
16 & 30 & 45.36\end{array}$ & $\begin{array}{l}-0.02 \\
+0.07 \\
-0.14 \\
+0.05 \\
-0.13\end{array}$ & $\begin{array}{l}69.60 \\
69.77 \\
69.86 \\
70.13 \\
70.26\end{array}$ & $\begin{array}{r}0.00 \\
+0.07 \\
+0.05 \\
+0.03 \\
-0.02\end{array}$ & $:$ & $\begin{array}{rrr}-20 & 37 & 36.6 \\
-20 & 49 & 23.8 \\
-21 & 0 & 48.3 \\
-21 & 32 & 42.4 \\
-21 & 51 & 57.6\end{array}$ & $\begin{array}{l}-1.0 \\
+0.8 \\
+1.9 \\
+1.9 \\
+0.4\end{array}$ & $\begin{array}{ll}16 & 13.4 \\
16 & 14.0 \\
16 & 13.7 \\
16 & 14.2 \\
16 & 15.5\end{array}$ & $\begin{array}{l}-0.9 \\
-0.4 \\
-0.9 \\
-0.9 \\
+0.1\end{array}$ \\
\hline $\begin{array}{r}5.0 \\
6.0 \\
7.0 \\
20.0 \\
21.0\end{array}$ & $\begin{array}{l}\text { M. } \\
\text { Hi. } \\
\text { P. } \\
\text { Hil. } \\
\text { P. }\end{array}$ & & $:$ & $\begin{array}{rrr}16 & 43 & 46.58 \\
16 & 48 & 8.18 \\
16 & 52 & 30.35 \\
17 & 49 & 52.13 \\
17 & 54 & 18.67\end{array}$ & $\begin{array}{l}-0.02 \\
+0.04 \\
+0.12 \\
-0.05 \\
+0.10\end{array}$ & $\begin{array}{l}70.48 \\
70.60 \\
70.74 \\
71.20 \\
71.26\end{array}$ & $\begin{array}{l}-0.04 \\
+0.01 \\
+0.08 \\
-0.04 \\
+0.01\end{array}$ & $:$ & $\begin{array}{lll}-22 & 17 & 38.9 \\
-22 & 25 & 20.3 \\
-22 & 32 & 37.3 \\
-23 & 25 & 47.1 \\
-23 & 26 & 37.9\end{array}$ & $\begin{array}{l}+0.6 \\
+1.4 \\
+0.7 \\
+1.3 \\
+0.8\end{array}$ & $\begin{array}{ll}16 & 15.5 \\
16 & 15.2 \\
16 & 14.8 \\
16 & 15.7 \\
16 & 17.5\end{array}$ & $\begin{array}{r}-0.3 \\
-0.8 \\
-1.3 \\
-1.7 \\
0.0\end{array}$ \\
\hline $\begin{array}{l}24.0 \\
26.0 \\
31.0\end{array}$ & $\begin{array}{l}\mathrm{Hl} . \\
\mathrm{M} . \\
\mathrm{M} .\end{array}$ & & $:$ & $\begin{array}{rrr}18 & 7 & 38.06 \\
18 & 16 & 31.09 \\
18 & 38 & 41.73\end{array}$ & $\begin{array}{l}-0.05 \\
-0.04 \\
-0.02\end{array}$ & $\begin{array}{l}71.34 \\
71.18 \\
71.08\end{array}$ & $\begin{array}{l}+0.08 \\
-0.07 \\
-0.04\end{array}$ & $:$ & $\begin{array}{lrr}-23 & 26 & 18.9 \\
-23 & 23 & 45.9 \\
-23 & 9 & 8.6\end{array}$ & $\begin{array}{l}+0.9 \\
-0.1 \\
-0.4\end{array}$ & $\begin{array}{ll}16 & 18.5 \\
16 & 15.4 \\
16 & 17.0\end{array}$ & $\begin{array}{l}+0.8 \\
-2.4 \\
-0.8\end{array}$ \\
\hline $\begin{array}{l}1908 \\
\text { Jan. }\end{array}$ & M. & & $\cdot$ & $18 \quad 47 \quad 32.49$ & -0.02 & 70.98 & -0.06 & $\cdot$ & $-23 \quad 0 \quad 1.6$ & +0.3 & 1616.7 & -1.1 \\
\hline $\begin{array}{r}3.0 \\
6.0 \\
9.0 \\
10.0 \\
11.0\end{array}$ & $\begin{array}{l}\mathrm{P} . \\
\mathrm{M} . \\
\mathrm{M} . \\
\mathrm{M} .\end{array}$ & & $:$ & $\begin{array}{rrr}18 & 51 & 57.40 \\
19 & 5 & 9.88 \\
19 & 18 & 18.05 \\
19 & 22 & 39.96 \\
19 & 27 & 0.94\end{array}$ & $\begin{array}{l}-0.02 \\
+0.02 \\
-0.14 \\
+0.06 \\
-0.09\end{array}$ & $\begin{array}{l}71.02 \\
70.79 \\
70.65 \\
70.56 \\
70.47\end{array}$ & $\begin{array}{r}+0.03 \\
-0.03 \\
+0.01 \\
0.00 \\
-0.01\end{array}$ & $:$ & $\begin{array}{rrr}\ddot{2} 2 & \ddot{36} & 17.5 \\
-22 & 13 & 49.2 \\
-22 & 5 & 25.8 \\
-21 & 56 & 38.9\end{array}$ & $\begin{array}{l}+\ddot{2} .2 \\
+1.4 \\
+2.3 \\
+0.9\end{array}$ & $\begin{array}{ll}16 & 16.1 \\
16 & 16.9 \\
16 & 16.5 \\
16 & 16.4\end{array}$ & $\begin{array}{l}-1.7 \\
-0.9 \\
-1.2 \\
-1.3\end{array}$ \\
\hline $\begin{array}{l}14.0 \\
15.0 \\
17.0 \\
20.0 \\
21.0\end{array}$ & $\begin{array}{l}\mathrm{P} . \\
\mathrm{M} . \\
\mathrm{P} \\
\mathrm{Hl} \\
\mathrm{M} .\end{array}$ & E. & $\dot{:}$ & $\begin{array}{rrr}19 & 40 & 0.90 \\
19 & 44 & 19.42 \\
19 & 52 & 54.75 \\
20 & 5 & 42.26 \\
20 & 9 & 56.51\end{array}$ & $\begin{array}{l}+0.12 \\
+0.02 \\
+0.13 \\
+0.09 \\
-0.07\end{array}$ & $\begin{array}{l}70.34 \\
70.08 \\
70.04 \\
69.58 \\
69.56\end{array}$ & $\begin{array}{r}+0.10 \\
-0.07 \\
+0.07 \\
-0.08 \\
0.00\end{array}$ & : & $\begin{array}{rrr}-21 & 27 & 40.6 \\
-21 & 17 & 11.6 \\
-20 & 54 & 59.0 \\
-20 & 18 & 43.2 \\
-20 & 5 & 51.8\end{array}$ & $\begin{array}{l}+1.3 \\
+1.0 \\
+1.8 \\
+1.3 \\
+1.0\end{array}$ & $\begin{array}{ll}16 & 16.9 \\
16 & 15.4 \\
16 & 16.5 \\
16 & 14.7 \\
16 & 15.5\end{array}$ & $\begin{array}{l}-0.7 \\
-2.1 \\
-0.9 \\
-2.4 \\
-1.5\end{array}$ \\
\hline
\end{tabular}




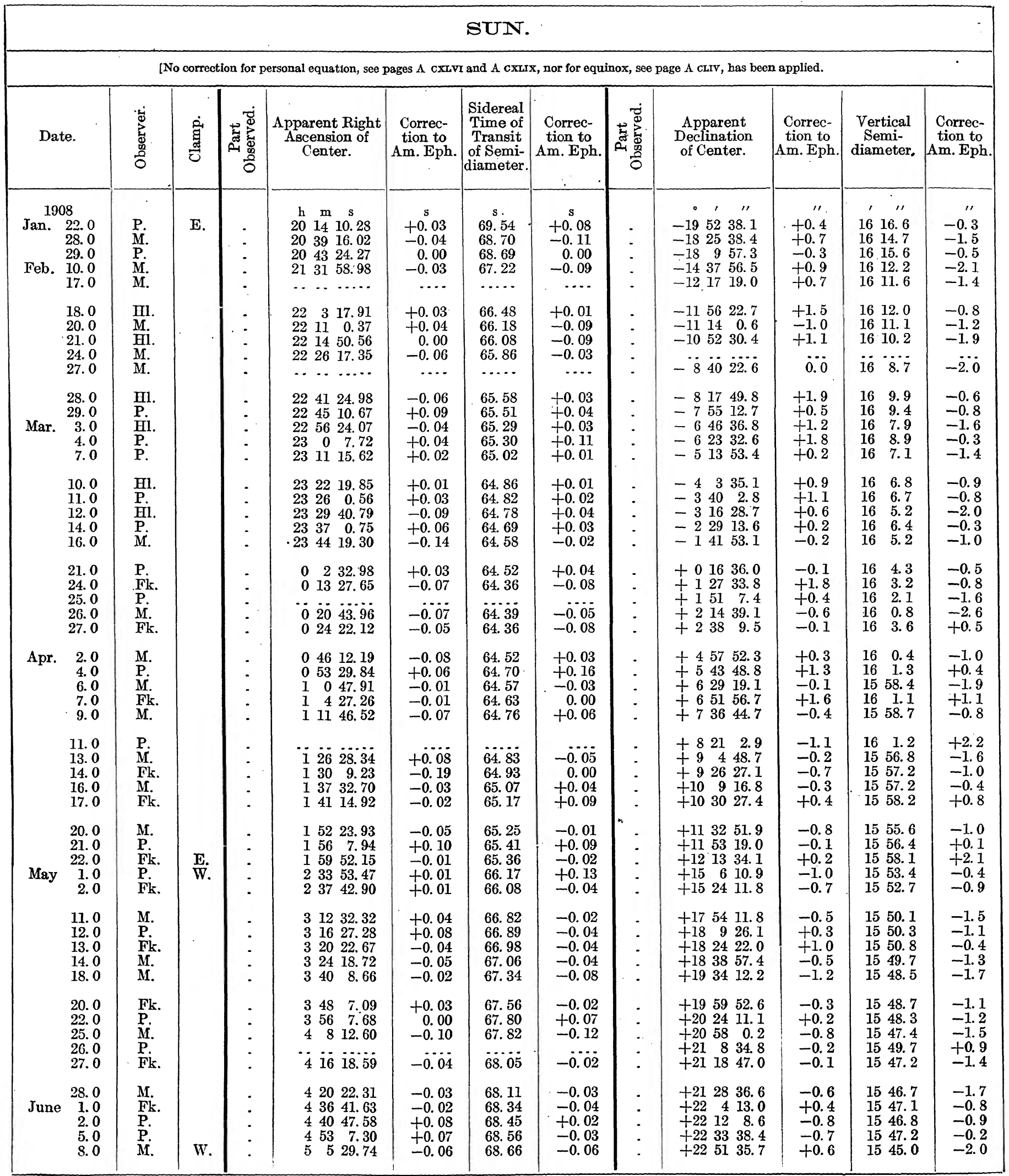


SUN.

[No correction for personal equation, see pages A cxLVI and A cxtrx, nor for equinox, see page A cLIv, has been applied.]

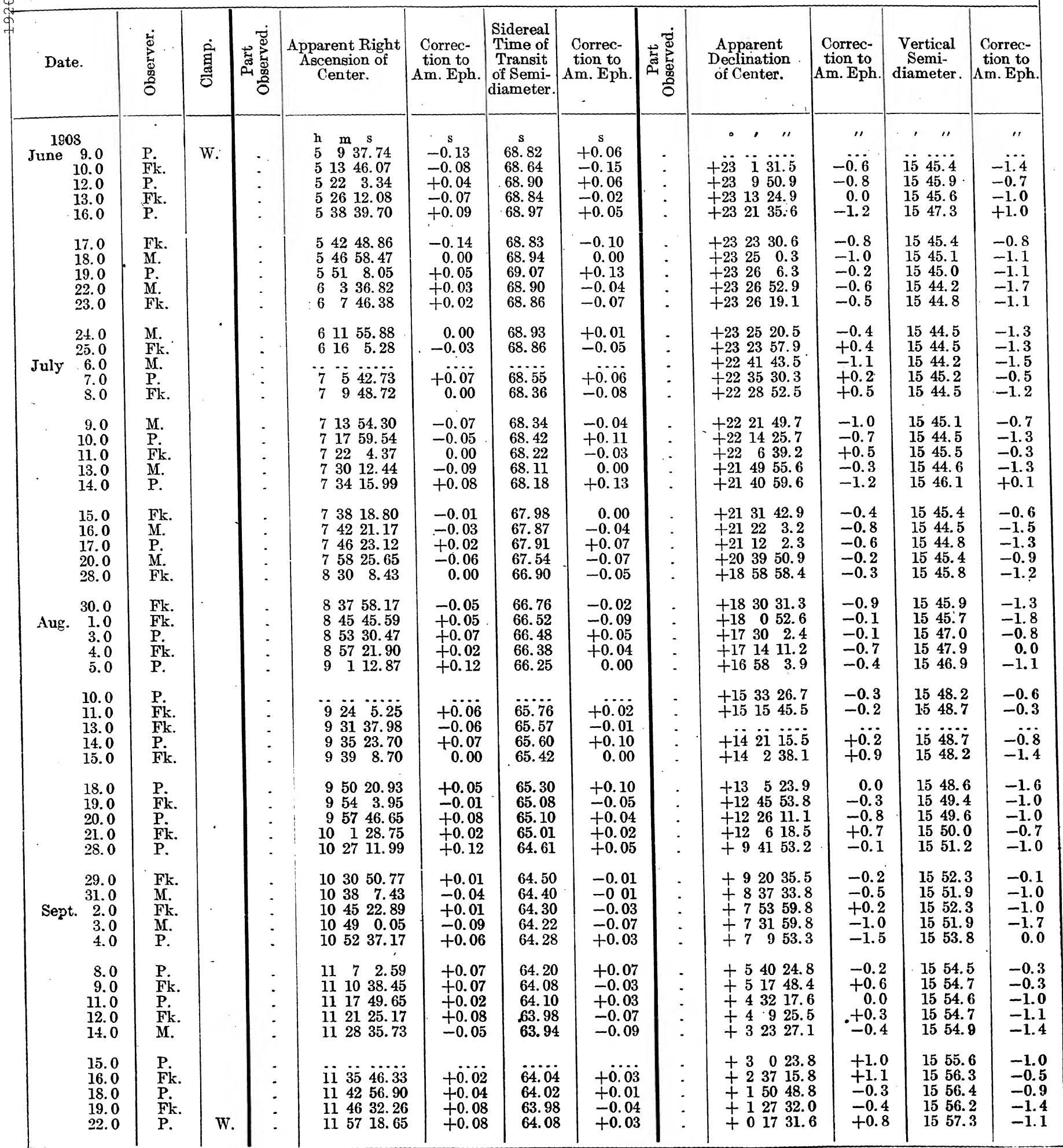

$29297^{\circ}-20-31$ 


\begin{tabular}{|c|c|c|c|c|c|c|c|c|c|c|c|c|}
\hline \multicolumn{13}{|c|}{ SUN. } \\
\hline \multicolumn{13}{|c|}{ [No correction for personal equation, see pages A cxuvs and A cxurx, nor for equinox, see page A curv, has been applied.] } \\
\hline Date. & $\begin{array}{l}\text { 离 } \\
\text { on } \\
\text { o }\end{array}$ & 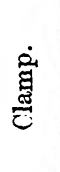 & 㺼 & $\begin{array}{c}\text { Apparent Right } \\
\text { Ascension of } \\
\text { Center. }\end{array}$ & $\begin{array}{c}\text { Correc- } \\
\text { tion to } \\
\text { Am. Eph. }\end{array}$ & $\begin{array}{c}\text { Sidereal } \\
\text { Time of } \\
\text { Transit } \\
\text { of Semi- } \\
\text { diameter. }\end{array}$ & $\begin{array}{c}\text { Correc- } \\
\text { tion to } \\
\text { Am. Eph. }\end{array}$ & 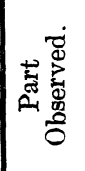 & $\begin{array}{l}\text { Apparent } \\
\text { Declination } \\
\text { of Center. } \\
i\end{array}$ & $\begin{array}{c}\text { Correc- } \\
\text { tion to } \\
\text { Am. Eph. }\end{array}$ & $\begin{array}{c}\text { Vertical } \\
\text { Semi- } \\
\text { diameter. }\end{array}$ & $\mid$\begin{tabular}{c|} 
Correc- \\
tion to \\
Am. Eph.
\end{tabular} \\
\hline 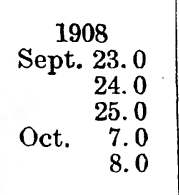 & $\begin{array}{l}\text { Fk. } \\
\text { M. } \\
\text { P. } \\
\text { L. } \\
\text { M. }\end{array}$ & w. & $:$ & $\begin{array}{ccc}\mathrm{h} & \mathrm{m} & \mathrm{s} \\
12 & 0 & 54.24 \\
12 & 4 & 30.06 \\
12 & 8 & 6.10 \\
12 & 51 & 35.72 \\
12 & 55 & 15.17\end{array}$ & $\begin{array}{c}s \\
0.00 \\
+0.01 \\
+0.08 \\
+0.09 \\
-0.03\end{array}$ & $\begin{array}{c}\text { s } \\
64.04 \\
64.06 \\
64.16 \\
64.66 \\
64.66\end{array}$ & $\begin{array}{c}\text { s. } \\
-0.03 \\
-0.03 \\
+0.04 \\
+0.03 \\
-0.03\end{array}$ & : & 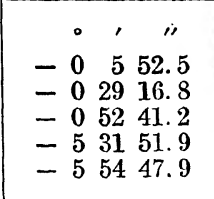 & $\begin{array}{c}11 \\
-0.1 \\
-0.2 \\
-0.2 \\
+0.5 \\
+0.7\end{array}$ & $\begin{array}{cc}\prime & \prime \prime \\
15 & 57.6 \\
15 & 56.9 \\
15 & 58.1 \\
16 & 2.1 \\
16 & 1.6\end{array}$ & $\begin{aligned} & " 1 \\
&-1.0 \\
&-2.0 \\
&-1.1 \\
&-0.5 \\
&-1.2\end{aligned}$ \\
\hline $\begin{array}{l}12.0 \\
13.0 \\
14.0 \\
15.0 \\
16.0\end{array}$ & $\begin{array}{l}\text { M. } \\
\text { P. } \\
\text { M. } \\
\text { M. }\end{array}$ & & : & $\begin{array}{rrr}13 & 9 & 57.90 \\
13 & 13 & 40.01 \\
13 & 17 & 22.34 \\
13 & 21 & 5.54 \\
13 & 24 & 49.10\end{array}$ & $\begin{array}{l}-0.10 \\
+0.08 \\
-0.06 \\
+0.11 \\
+0.07\end{array}$ & $\begin{array}{l}64.94 \\
65.18 \\
65.08 \\
65.27 \\
65.24\end{array}$ & $\begin{array}{l}-0.03 \\
+0.13 \\
-0.05 \\
+0.06 \\
-0.05\end{array}$ & $:$ & $\begin{array}{l}-72543.6 \\
=74814.1 \\
=81035.3 \\
=83252.8 \\
-8552.3\end{array}$ & $\begin{array}{l}+0.4 \\
-0.5 \\
+1.4 \\
+0.3 \\
+0.1\end{array}$ & $\begin{array}{ll}16 & 2.4 \\
16 & 3.6 \\
16 & 4.9 \\
16 & 3.2 \\
16 & 3.9\end{array}$ & $\begin{array}{l}-1.5 \\
-0.6 \\
+0.4 \\
-1.6 \\
-1.1\end{array}$ \\
\hline $\begin{array}{l}17.0 \\
19.0 \\
20.0 \\
23.0 \\
26.0\end{array}$ & $\begin{array}{l}\text { P. } \\
\text { M. } \\
\text { P. } \\
\text { P. }\end{array}$ & & : & $\begin{array}{rrr}13 & 28 & 33.34 \\
13 & 36 & 3.50 \\
13 & 39 & 49.72 \\
13 & 51 & 12.02 \\
14 & 2 & 40.54\end{array}$ & $\begin{array}{l}+0.11 \\
-0.03 \\
+0.08 \\
+0.08 \\
-0.01\end{array}$ & $\begin{array}{l}65.42 \\
65.52 \\
65.70 \\
65.94 \\
66.18\end{array}$ & $\begin{array}{l}+0.04 \\
-0.04 \\
+0.05 \\
+0.01 \\
-0.07\end{array}$ & : & $\begin{array}{lrr}-9 & 17 & 3.9 \\
-10 & 0 & 44.0 \\
-10 & 22 & 20.4 \\
-11 & 26 & 14.9 \\
-12 & 28 & 36.9\end{array}$ & $\begin{array}{l}+0.2 \\
-0.6 \\
-0.1 \\
-0.1 \\
-0.3\end{array}$ & $\begin{array}{ll}16 & 4.0 \\
16 & 4.8 \\
16 & 4.6 \\
16 & 6.0 \\
16 & 6.0\end{array}$ & $\begin{array}{l}-1.3 \\
-1.0 \\
-1.5 \\
-0.9 \\
-1.7\end{array}$ \\
\hline $\begin{array}{r}27.0 \\
30.0 \\
31.0 \\
\text { Nov. } 2.0 \\
3.0\end{array}$ & $\begin{array}{l}\text { P. } \\
\text { P. } \\
\text { M. } \\
\text { P. }\end{array}$ & & : & $\begin{array}{rrr}14 & 6 & 31.57 \\
14 & 18 & 9.01 \\
14 & 22 & 2.98 \\
14 & 29 & 53.25 \\
14 & 33 & 49.61\end{array}$ & $\begin{array}{l}+0.05 \\
+0.08 \\
+0.08 \\
+0.06 \\
+0.08\end{array}$ & $\begin{array}{l}66.36 \\
66.68 \\
66.86 \\
67.00 \\
67.18\end{array}$ & $\begin{array}{r}0.00 \\
0.00 \\
+0.07 \\
-0.02 \\
+0.04\end{array}$ & . & $\begin{array}{rrr} & & 49 \\
-12 & 49 & 1.9 \\
-13 & 49 & 2.4 \\
-14 & 8 & 36.2 \\
-14 & 27 \\
-15 & 5 & 53.9\end{array}$ & $\begin{array}{l}-0.7 \\
-0.7 \\
-0.3 \\
+0.7 \\
+0.7\end{array}$ & $\begin{array}{ll}16 & 7.9 \\
16 & 9.4 \\
16 & 8.4 \\
16 & 7.5 \\
16 & 8.6\end{array}$ & $\begin{array}{r}0.0 \\
+0.7 \\
-0.6 \\
-2.0 \\
-1.1\end{array}$ \\
\hline $\begin{array}{r}5.0 \\
6.0 \\
9.0 \\
12.0 \\
12.0\end{array}$ & $\begin{array}{l}\text { Mr. } \\
\text { P. } \\
\text { M. } \\
\text { M. }\end{array}$ & & : & $\begin{array}{lll}14 & 41 & 44.55 \\
14 & 45 & 43.46 \\
14 & 57 & 44.60 \\
15 & 1 & 46.86 \\
15 & 9 & 53.50\end{array}$ & $\begin{array}{l}-0.03 \\
+0.12 \\
-0.04 \\
+0.09 \\
-0.12\end{array}$ & $\begin{array}{l}67.37 \\
67.53 \\
67.80 \\
68.00 \\
68.24\end{array}$ & $\begin{array}{l}-0.01 \\
+0.03 \\
-0.06 \\
+0.02 \\
+0.02\end{array}$ & : & $\begin{array}{llll}-15 & 42 & 52.2 \\
-16 & 0 & 58.6 \\
-16 & 53 & 38.7 \\
-17 & 10 & 37.4 \\
-17 & 43 & 44.1\end{array}$ & $\begin{array}{l}+0.9 \\
+0.4 \\
+0.2 \\
+0.8 \\
-0.3\end{array}$ & $\begin{array}{rr}16 & 9.5 \\
16 & 9.5 \\
16 & 9.2 \\
16 & 10.0 \\
16 & 11.5\end{array}$ & $\begin{array}{l}-0.7 \\
-1.0 \\
-2.0 \\
-1.4 \\
-0.3\end{array}$ \\
\hline $\begin{array}{l}13.0 \\
11.0 \\
17.0 \\
18.0 \\
19.0\end{array}$ & $\begin{array}{l}\mathrm{P} . \\
\text { M. } \\
\mathrm{P} . \\
\mathrm{L} .\end{array}$ & & : & $\begin{array}{lll}15 & 13 & 58.48 \\
15 & 26 & 17.69 \\
15 & 30 & 25.89 \\
15 & 34 & 35.05 \\
15 & 38 & 44.70\end{array}$ & $\begin{array}{l}+0.16 \\
-0.03 \\
-0.01 \\
+0.12 \\
-0.10\end{array}$ & & $\begin{array}{r}+0.16 \\
0.00 \\
+0.09 \\
+0.01 \\
-0.04\end{array}$ & 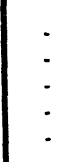 & $\begin{array}{lll}-17 & 59 & 50.2 \\
-18 & 46 & 1.5 \\
-19 & 1 & 0.9 \\
-19 & 15 & 27.3 \\
-19 & 29 & 33.8\end{array}$ & $\begin{array}{l}-1.0 \\
+0.1 \\
-1.1 \\
+0.2 \\
+0.6\end{array}$ & $\begin{array}{ll}16 & 11.7 \\
16 & 10.7 \\
16 & 13.9 \\
16 & 12.3 \\
16 & 11.9\end{array}$ & $\begin{array}{l}-0.3 \\
-2.0 \\
+1.0 \\
-0.8 \\
-1.4\end{array}$ \\
\hline $\begin{array}{r}20.0 \\
21.0 \\
27.0 \\
30.0 \\
\text { Dec. } \quad 1.0\end{array}$ & $\begin{array}{l}\text { P. } \\
\text { P. } \\
\text { M. } \\
\text { P. }\end{array}$ & & : & $\begin{array}{rrr}15 & 42 & 55.65 \\
15 & 47 & 7.08 \\
16 & 12 & 32.81 \\
16 & 25 & 25.45 \\
16 & 29 & 44.51\end{array}$ & $\begin{array}{l}+0.14 \\
+0.04 \\
-0.01 \\
-0.06 \\
+0.12\end{array}$ & $\begin{array}{l}69.20 \\
69.32 \\
70.03 \\
70.10 \\
70.19\end{array}$ & $\begin{array}{l}+0.06 \\
+0.07 \\
+0.14 \\
-0.07 \\
-0.07\end{array}$ & $:$ & $\begin{array}{lll}-19 & 43 & 20.7 \\
-19 & 56 & 44.5 \\
-21 & 922.3 \\
-21 & 40 & 20.0 \\
-21 & 49 & 51.2\end{array}$ & $\begin{array}{l}-0.3 \\
+0.5 \\
-1.0 \\
+0.4 \\
-0.5\end{array}$ & $\begin{array}{ll}16 & 12.5 \\
16 & 13.1 \\
16 & 16.2 \\
16 & 12.5 \\
16 & 13.8\end{array}$ & $\begin{array}{l}-1.0 \\
-0.5 \\
+1.5 \\
-2.7 \\
-1.6\end{array}$ \\
\hline $\begin{array}{r}2.0 \\
3.0 \\
8.0 \\
9.0 \\
16.0\end{array}$ & $\begin{array}{l}\text { M. } \\
\text { P. } \\
\text { L. } \\
\text { L. }\end{array}$ & & $:$ & $\begin{array}{rrr}16 & 34 & 3.84 \\
16 & 38 & 23.91 \\
17 & 0 & 12.74 \\
17 & 4 & 36.19 \\
\ldots & . . & \cdots\end{array}$ & $\begin{array}{l}-0.05 \\
-0.07 \\
-0.04 \\
+0.14\end{array}$ & & $\begin{array}{l}+0.05 \\
+0.13 \\
+0.10 \\
+0.07\end{array}$ & : & $\begin{array}{lll}-21 & 58 & 54.9 \\
-22 & 7 & 36.8 \\
-22 & 44 & 22.8 \\
-22 & 50 & 23.5 \\
-23 & 19 & 52.3\end{array}$ & $\begin{array}{r}+0.7 \\
-1.6 \\
0.0 \\
+1.3 \\
+1.3\end{array}$ & $\begin{array}{ll}16 & 15.1 \\
16 & 15.4 \\
16 & 15.9 \\
16 & 16.0 \\
16 & 16.0\end{array}$ & $\begin{array}{l}-0.5 \\
-0.3 \\
-0.5 \\
-0.5 \\
-1.2\end{array}$ \\
\hline $\begin{array}{l}19.0 \\
23.0 \\
26.0 \\
28.0 \\
29.0\end{array}$ & $\begin{array}{l}\text { L. } \\
\text { L. } \\
\text { L. } \\
\text { M. } \\
\text { P. }\end{array}$ & & $:$ & $\begin{array}{lrr}17 & 48 & 48.58 \\
18 & 6 & 35.28 \\
18 & 19 & 54.96 \\
18 & 28 & 47.62 \\
18 & 33 & 13.69\end{array}$ & $\begin{array}{r}+0.07 \\
+0.19 \\
0.00 \\
+0.04 \\
+0.11\end{array}$ & $\begin{array}{l}71.29 \\
71.27 \\
71.30 \\
71.12 \\
71.21\end{array}$ & $\begin{array}{l}+0.06 \\
+0.01 \\
+0.07 \\
+0.07 \\
+0.05\end{array}$ & $\cdot$ & $\begin{array}{lll}-23 & 25 & 34.3 \\
-23 & 26 & 33.1 \\
-23 & 22 & 19.9 \\
-23 & 17 & 11.1 \\
-23 & 13 & 52.9\end{array}$ & $\begin{array}{r}0.0 \\
+0.5 \\
+0.9 \\
-0.2 \\
+1.0\end{array}$ & 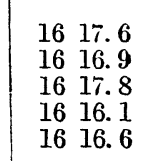 & $\begin{array}{r}+0.2 \\
-0.7 \\
0.0 \\
-1.7 \\
-1.2\end{array}$ \\
\hline 31.0 & M. & & - & 1842.4 .71 & -0.08 & 71.08 & -0.01 & - & $-23 \quad 555.9$ & +0.2 & 1617.2 & -0.7 \\
\hline \begin{tabular}{r}
\multicolumn{2}{c}{1909} \\
Jan. $\quad 2.0$ \\
4.0 \\
6.0 \\
18.0 \\
19.0
\end{tabular} & $\begin{array}{l}\mathrm{L} . \\
\mathrm{M} . \\
\mathrm{L} . \\
\mathrm{M} . \\
\mathrm{P} .\end{array}$ & & : & $\begin{array}{cccc}\text { 18 } & 50 & 54.83 \\
\text { ig } & \because 8 & 30.16 \\
20 & 0 & 30.90 \\
20 & 4 & 41.46\end{array}$ & $\begin{array}{l}+0.04 \\
-0.01 \\
-0.01 \\
+0.07\end{array}$ & $\begin{array}{l}71.14 \\
70.70 \\
69.74 \\
69.78\end{array}$ & $\begin{array}{r}+0.15 \\
-0.03 \\
-0.05 \\
+0.09\end{array}$ & 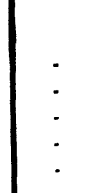 & $\begin{array}{rrr}-22 & 56 & 6.3 \\
-22 & 44 & 27.8 \\
-22 & 31 & 1.4 \\
-20 & 34 & 11.5 \\
-20 & 21 & 46.9\end{array}$ & $\begin{array}{r}+0.9 \\
+1.0 \\
+0.8 \\
0.0 \\
+1.1\end{array}$ & $\begin{array}{ll}16 & 17.4 \\
16 & 16.7 \\
16 & 16.0 \\
16 & 15.8 \\
16 & 16.8\end{array}$ & $\begin{array}{l}-0.5 \\
-1.2 \\
-1.9 \\
-1.4 \\
-0.3\end{array}$ \\
\hline $\begin{array}{l}20.0 \\
22.0 \\
25.0 \\
26.0 \\
27.0\end{array}$ & $\begin{array}{l}\mathrm{L} . \\
\mathrm{P} . \\
\mathrm{M} . \\
\mathrm{L} . \\
\mathrm{P} .\end{array}$ & W. & $:$ & $\begin{array}{rrr}20 & 8 & 56.25 \\
20 & 17 & 23.54 \\
20 & 29 & 58.24 \\
20 & 34 & 8.46 \\
20 & 38 & 17.57\end{array}$ & $\begin{array}{l}+0.11 \\
+0.17 \\
-0.07 \\
+0.13 \\
+0.05\end{array}$ & $\begin{array}{l}69.63 \\
69.35 \\
68.95 \\
69.01 \\
68.86\end{array}$ & $\begin{array}{l}+0.04 \\
-0.03 \\
-0.10 \\
+0.07 \\
+0.03\end{array}$ & : & $\begin{array}{lrr}-20 & 9 & 1.3 \\
-19 & 42 & 19.5 \\
-18 & 59 & 36.7 \\
-18 & 44 & 38.4 \\
-18 & 29 & 20.9\end{array}$ & $\begin{array}{l}+0.2 \\
+1.3 \\
-0.9 \\
+0.3 \\
+0.3\end{array}$ & $\begin{array}{ll}16 & 15.8 \\
16 & 14.8 \\
16 & 14.9 \\
16 & 16.2 \\
16 & 15.9\end{array}$ & $\begin{array}{l}-1.2 \\
-2.0 \\
-1.6 \\
-0.2 \\
-0.4\end{array}$ \\
\hline
\end{tabular}


SUN.

[No correction for personal equation, see pages A CXLVI and A cxLIx, nor for equinox, see page $A$ cLiv, has been applied.]

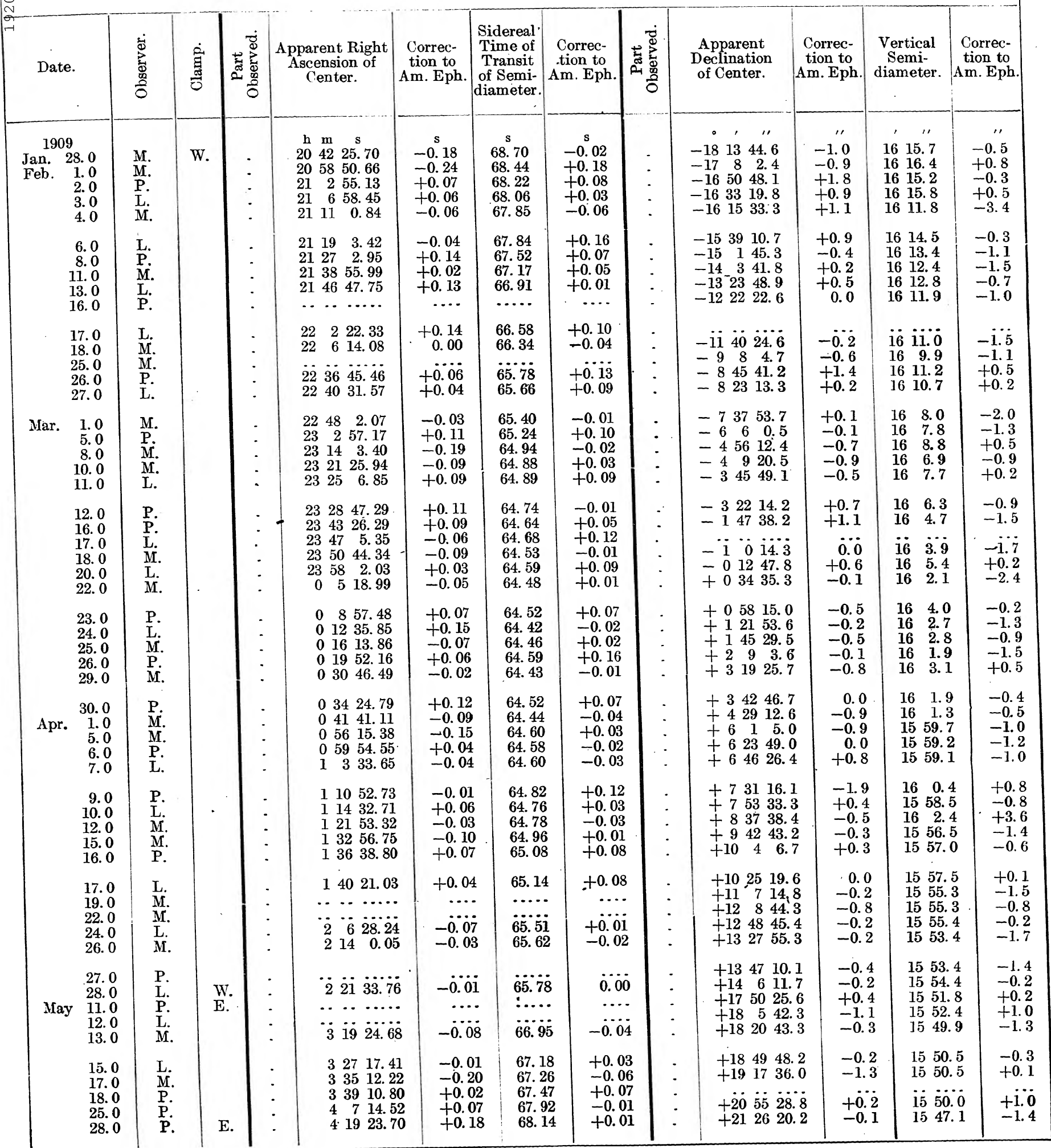

$29297^{\circ}-19-2$ 


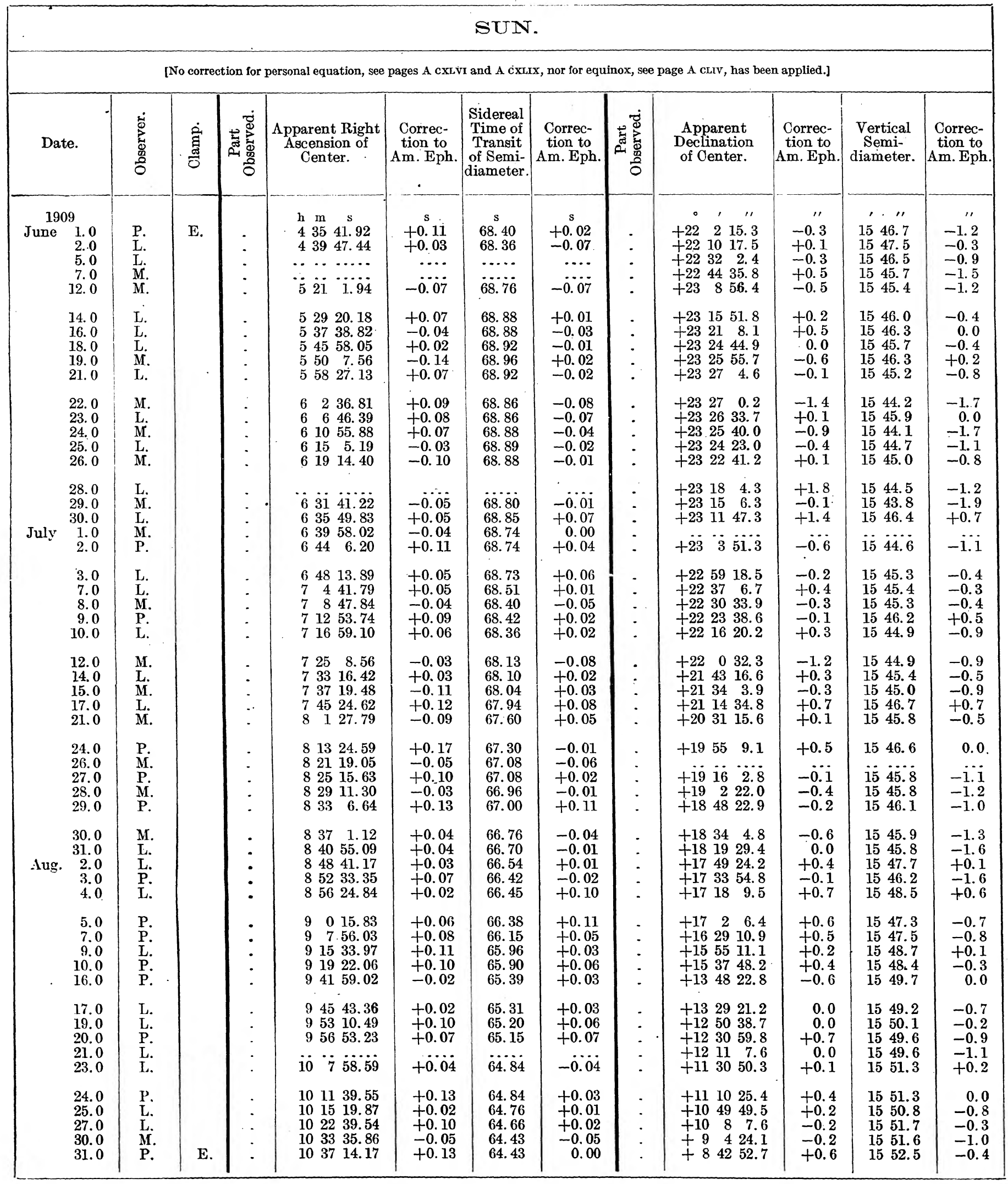


RESULTS OF OBSERVATIONS OF THE SUN, MOON, AND PLANETS.

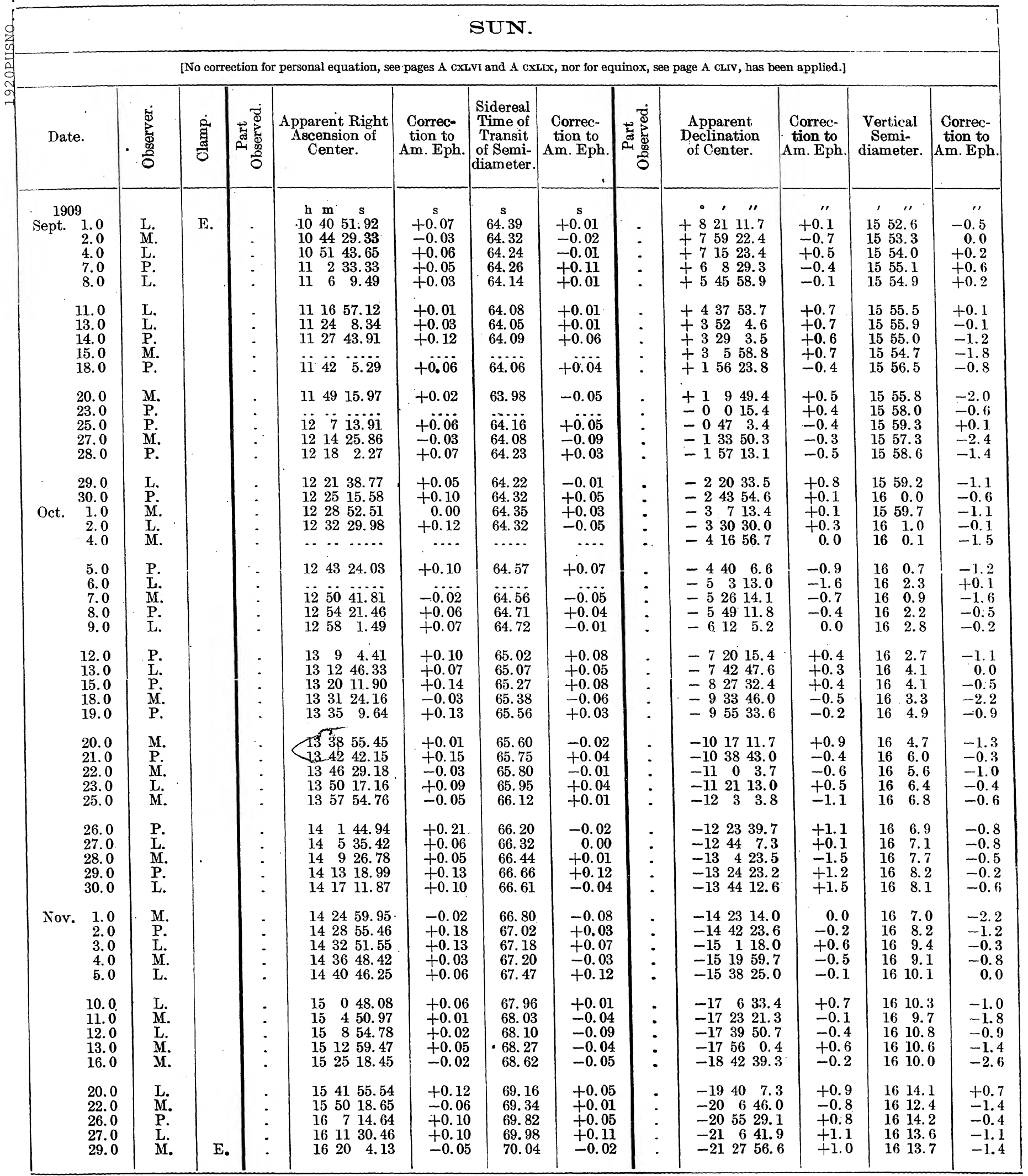




\begin{tabular}{|c|c|c|c|c|c|c|c|c|c|c|c|c|}
\hline \multicolumn{13}{|c|}{ SUN. } \\
\hline \multicolumn{13}{|c|}{ [No correction for personal equation, see pages A cxavi and A cxurx, nor for equinox, see page A crrv, has been applied.] } \\
\hline Date. & 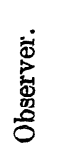 & 家 & 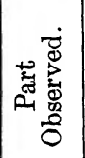 & $\begin{array}{l}\text { Apparent Right } \\
\text { Ascension of } \\
\text { Center. }\end{array}$ & $\begin{array}{c}\text { Correc- } \\
\text { tion to } \\
\text { Am. Eph. }\end{array}$ & $\begin{array}{l}\text { Sidereal } \\
\text { Time of } \\
\text { Transit } \\
\text { of Semi- } \\
\text { diameter. }\end{array}$ & $\begin{array}{c}\text { Correc- } \\
\text { tion to } \\
\text { Am. Eph. }\end{array}$ & 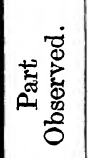 & $\begin{array}{l}\text { Apparent } \\
\text { Declination } \\
\text { of Center. }\end{array}$ & $\begin{array}{c}\text { Correc- } \\
\text { tion to } \\
\text { Am. Eph. }\end{array}$ & $\begin{array}{c}\text { Vertical } \\
\text { Semi- } \\
\text { diameter. }\end{array}$ & $\begin{array}{l}\text { Correc- } \\
\text { tion to } \\
\text { Am. Eph. }\end{array}$ \\
\hline $\begin{array}{c}1909 \\
\text { Nov. } 30.0 \\
\text { Dec. } 1.0 \\
2.0 \\
3.0 \\
4.0\end{array}$ & $\begin{array}{l}\text { P. } \\
\text { L. } \\
\text { M. } \\
\text { P. }\end{array}$ & E. & . & \begin{tabular}{rrr}
$\mathrm{h}$ & $\mathrm{m}$ & \multicolumn{1}{c}{$\mathrm{s}$} \\
16 & 24 & 22.24 \\
16 & 28 & 40.80 \\
16 & 33 & 0.02 \\
16 & 37 & 20.26 \\
16 & 41 & 40.76
\end{tabular} & $\begin{array}{l}\mathrm{s} \\
+0.10 \\
+0.01 \\
+0.09 \\
+0.19 \\
+0.10\end{array}$ & $\begin{array}{c}\mathrm{s} \\
70.18 \\
70.34 \\
70.30 \\
70.43 \\
70.46\end{array}$ & $\begin{array}{c}\mathrm{s} \\
+0.03 \\
+0.10 \\
-0.03 \\
+0.02 \\
-0.03\end{array}$ & $:$ & $\begin{array}{rrr}\circ & , & \prime \prime \\
-\ddot{21} & \ddot{4} & 33.4 \\
-21 & 56 & 45.7 \\
-22 & 5 & 30.8 \\
-22 & 13 & 51.3\end{array}$ & $\begin{array}{r} \\
\quad \cdots \\
+1.2 \\
+0.1 \\
+0.8 \\
+0.7\end{array}$ & $\begin{array}{cc}, & \prime \prime \\
16 & 13.9 \\
16 & 15.0 \\
16 & 14.8 \\
16 & 15.0\end{array}$ & $\begin{array}{r}. \prime \\
-0.5 \\
-0.5 \\
-0.9 \\
-0.8\end{array}$ \\
\hline $\begin{array}{r}9.0 \\
10.0 \\
11.0 \\
14.0 \\
15.0\end{array}$ & $\begin{array}{l}\text { L. } \\
\text { M. } \\
\text { L. } \\
\text { M. } \\
\text { L. }\end{array}$ & & : & $\begin{array}{ccc}17 & 3 & 32.03 \\
17 & 7 & 55.76 \\
\ldots & \ldots & \ldots . . \\
17 & 0 & \ldots \\
17 & 30 & 0.38\end{array}$ & $\begin{array}{r}-0.03 \\
-0.07 \\
\ldots \ldots \\
-0.01\end{array}$ & $\begin{array}{l}70.95 \\
70.94 \\
\cdots \ldots . \\
71.14\end{array}$ & $\begin{array}{r}+0.12 \\
+0.05 \\
\ldots \ldots \\
+0.02\end{array}$ & : & $\begin{array}{lll}-22 & 48 & 59.9 \\
-22 & 54 & 43.9 \\
-22 & 59 & 56.8 \\
-23 & 12 & 57.7 \\
-23 & 16 & 21.6\end{array}$ & $\begin{array}{l}+1.9 \\
-0.2 \\
+1.8 \\
+0.6 \\
+1.2\end{array}$ & $\begin{array}{ll}16 & 16.2 \\
16 & 15.9 \\
16 & 16.5 \\
16 & 16.5 \\
16 & 17.2\end{array}$ & $\begin{array}{l}-0.2 \\
-0.6 \\
-0.1 \\
-0.4 \\
+0.2\end{array}$ \\
\hline $\begin{array}{l}16.0 \\
17.0 \\
18.0 \\
21.0 \\
22.0\end{array}$ & $\begin{array}{l}\text { M. } \\
\text { L. } \\
\text { M. } \\
\text { L. }\end{array}$ & & : & $\begin{array}{lll}17 & 34 & 26.18 \\
17 & 38 & 52.24 \\
17 & 43 & 18.34 \\
17 & 56 & 37.76 \\
\ldots & \ldots & \ldots . .\end{array}$ & $\begin{array}{c}-0.02 \\
+0.03 \\
-0.06 \\
+0.10 \\
\ldots \ldots\end{array}$ & $\begin{array}{l}71.08 \\
71.21 \\
71.21 \\
71.32 \\
\cdots \cdots\end{array}$ & $\begin{array}{r}-0.07 \\
+0.03 \\
+0.01 \\
+0.07 \\
\quad \ldots .\end{array}$ & : & $\begin{array}{rrr}-23 & 19 & 19.1 \\
-23 & 21 & 46.6 \\
-23 & 23 & 47.4 \\
-23 & 26 & 57.7 \\
-23 & 27 & 6.0\end{array}$ & $\begin{array}{l}+0.1 \\
+1.0 \\
+0.5 \\
+1.7 \\
+0.7\end{array}$ & $\begin{array}{ll}16 & 15.9 \\
16 & 16.5 \\
16 & 15.4 \\
16 & 16.5 \\
16 & 16.9\end{array}$ & $\begin{array}{l}-1.2 \\
-0.7 \\
-1.9 \\
-1.0 \\
-0.7\end{array}$ \\
\hline $\begin{array}{l}23.0 \\
24.0 \\
28.0 \\
29.0 \\
30.0\end{array}$ & $\begin{array}{l}\text { M. } \\
\text { P. } \\
\text { L. } \\
\text { M. }\end{array}$ & & : & $\begin{array}{rrr}18 & 5 & 30.72 \\
18 & 9 & 57.26 \\
18 & 27 & 42.52 \\
18 & 32 & 8.31 \\
18 & 36 & 33.91\end{array}$ & $\begin{array}{r}-0.04 \\
0.00 \\
+0.15 \\
0.00 \\
-0.13\end{array}$ & $\begin{array}{l}71.22 \\
71.34 \\
71.24 \\
71.14 \\
71.18\end{array}$ & $\begin{array}{l}-0.04 \\
+0.08 \\
+0.03 \\
-0.04 \\
+0.04\end{array}$ & $\dot{.}$ & $\begin{array}{rrr}-23 & \ddot{2} & \ddot{56.8} \\
-23 & 17 & 55.4 \\
-23 & 14 & 45.1 \\
-23 & 11 & 7.2\end{array}$ & $\begin{array}{l}-\ddot{0.6} \\
+1.1 \\
+1.3 \\
+1.1\end{array}$ & $\begin{array}{ll}16 & 18.4 \\
16 & 17.6 \\
16 & 17.1 \\
16 & 16.0\end{array}$ & $\begin{array}{l}+0.7 \\
-0.2 \\
-0.7 \\
-1.9\end{array}$ \\
\hline 31.0 & P. & & . & 184059.51 & -0.02 & 71.22 & +0.12 & . & $-23 \quad 659.6$ & +2.7 & 1619.1 & +1.2 \\
\hline \begin{tabular}{r}
\multicolumn{2}{c}{1910} \\
Jan. \\
4.0 \\
7.0 \\
8.0
\end{tabular} & $\begin{array}{l}\mathrm{P} . \\
\mathrm{P} . \\
\mathrm{L} .\end{array}$ & & $\dot{.}$ & $\begin{array}{lll}18 & 58 & 38.84 \\
19 & 11 & 49.06 \\
19 & 16 & 11.36\end{array}$ & $\begin{array}{l} \\
+0.29 \\
+0.20 \\
-0.03\end{array}$ & $\begin{array}{l}70.94 \\
70.76 \\
70.66\end{array}$ & $\begin{array}{l}+0.02 \\
+0.02 \\
-0.01\end{array}$ & $\dot{.}$ & $\begin{array}{lrr}-22 & 46 & 1.4 \\
-22 & 25 & 31.0 \\
-22 & 17 & 47.1\end{array}$ & $\begin{array}{l}+1.1 \\
+1.2 \\
+1.6\end{array}$ & $\begin{array}{ll}16 & 17.5 \\
16 & 16.2 \\
16 & 18.2\end{array}$ & $\begin{array}{l}-0.3 \\
-1.6 \\
+0.5\end{array}$ \\
\hline $\begin{array}{l}10.0 \\
11.0 \\
12.0 \\
15.0 \\
25.0\end{array}$ & $\begin{array}{l}\text { M. } \\
\text { P. } \\
\text { L. } \\
\text { L. }\end{array}$ & & : & $\begin{array}{llll}19 & 24 & 54.90 \\
19 & 29 & 15.92 \\
19 & 33 & 36.22 \\
19 & 46 & 33.35 \\
20 & 28 & 57.03\end{array}$ & $\begin{array}{l}-0.01 \\
+0.09 \\
+0.05 \\
-0.03 \\
+0.13\end{array}$ & $\begin{array}{l}70.52 \\
70.46 \\
70.36 \\
70.17 \\
69.09\end{array}$ & $\begin{array}{l}-0.01 \\
+0.01 \\
-0.01 \\
+0.07 \\
+0.01\end{array}$ & $:$ & $\begin{array}{rrr}-22 & 1 & 3.4 \\
-21 & 72 & 32.0 \\
-21 & 11 & 40.9 \\
-19 & 3 & 13.0\end{array}$ & $\begin{array}{l}-0.2 \\
+2.0 \\
+0.6 \\
+0.7\end{array}$ & $\begin{array}{ll}16 & 16.5 \\
16 & 16.2 \\
16 & 16.7 \\
16 & 15.6\end{array}$ & $\begin{array}{r}-1.2 \\
-1.4 \\
-0.7 \\
-1.0\end{array}$ \\
\hline Feb. $\begin{array}{r}1.0 \\
2.0 \\
4.0 \\
5.0 \\
8.0\end{array}$ & $\begin{array}{l}\text { M. } \\
\text { P. } \\
\text { P. } \\
\text { P. }\end{array}$ & & $\dot{5}$ & $\begin{array}{rrr}20 & 57 & 50.40 \\
21 & 1 & 55.03 \\
21 & 10 & 1.42 \\
21 & 14 & 3.37 \\
21 & 26 & 4.65\end{array}$ & $\begin{array}{l}-0.04 \\
+0.16 \\
+0.12 \\
+0.05 \\
+0.05\end{array}$ & $\begin{array}{l}68.26 \\
68.22 \\
67.98 \\
67.92 \\
67.54\end{array}$ & $\begin{array}{l}-0.02 \\
+0.06 \\
+0.05 \\
+0.11 \\
+0.07\end{array}$ & $:$ & $\begin{array}{rrr}-17 & 12 & 15.7 \\
-16 & 55 & 7.6 \\
-16 & 19 & 59.7 \\
-16 & 2 & 0.9 \\
-15 & 6 & 23.6\end{array}$ & $\begin{array}{l}-0.4 \\
+0.6 \\
+1.0 \\
+0.4 \\
+1.2\end{array}$ & $\begin{array}{ll}16 & 14.0 \\
16 & 14.9 \\
16 & 15.8 \\
16 & 14.6 \\
16 & 13.7\end{array}$ & $\begin{array}{l}-1.6 \\
-0.6 \\
+0.6 \\
-0.4 \\
-0.8\end{array}$ \\
\hline $\begin{array}{r}18.0 \\
19.0 \\
25.0 \\
26.0 \\
\text { Mar. } \quad 4.0\end{array}$ & $\begin{array}{l}\text { P. } \\
\text { L. } \\
\text { P. } \\
\text { P. }\end{array}$ & & $\dot{5}$ & $\begin{array}{rrr}22 & 5 & 18.07 \\
22 & 9 & 9.30 \\
22 & 32 & 2.56 \\
22 & 35 & 49.34 \\
22 & 58 & 18.82\end{array}$ & $\begin{array}{r}+0.11 \\
+0.13 \\
0.00 \\
+0.02 \\
+0.09\end{array}$ & $\begin{array}{l}66.44 \\
66.35 \\
65.83 \\
65.62 \\
65.24\end{array}$ & $\begin{array}{l}+0.02 \\
+0.03 \\
+0.08 \\
+0.05 \\
+0.03\end{array}$ & $:$ & $\begin{array}{l}-114532.5 \\
-112419.7 \\
-91333.6 \\
-85114.5 \\
-63449.6\end{array}$ & $\begin{array}{l}+0.5 \\
+0.5 \\
+1.1 \\
+1.5 \\
+1.7\end{array}$ & $\begin{array}{lr}16 & 12.9 \\
16 & 11.8 \\
16 & 11.7 \\
16 & 8.3 \\
16 & 9.5\end{array}$ & $\begin{array}{l}+0.3 \\
-0.6 \\
+0.6 \\
-2.5 \\
+0.1\end{array}$ \\
\hline $\begin{array}{r}5.0 \\
7.0 \\
8.0 \\
9.0 \\
11.0\end{array}$ & $\begin{array}{l}\text { L. } \\
\text { M. } \\
\text { P. } \\
\text { L. } \\
\text { P. }\end{array}$ & & : & $\begin{array}{rrr}23 & 2 & 2.00 \\
23 & 9 & 27.18 \\
23 & 13 & 9.33 \\
\cdots & . & \cdots \\
\therefore . & \cdots & \cdots \\
\end{array}$ & $\begin{array}{r}+0.03 \\
-0.01 \\
+0.13 \\
\ldots \ldots \\
\cdots\end{array}$ & $\begin{array}{l}65.20 \\
65.10 \\
65.00 \\
\cdots \cdots \\
\cdots \cdots\end{array}$ & $\begin{array}{r}+0.06 \\
+0.07 \\
+0.03 \\
\ldots \ldots \\
\ldots\end{array}$ & $\dot{.}$ & $\begin{array}{l}-61144.7 \\
-52517.9 \\
-5156.9 \\
-43832.8 \\
-35133.5\end{array}$ & $\begin{array}{l}+0.7 \\
+0.5 \\
+1.0 \\
+0.6 \\
+0.4\end{array}$ & $\begin{array}{ll}16 & 8.6 \\
16 & 8.0 \\
16 & 8.3 \\
16 & 6.9 \\
16 & 5.2\end{array}$ & $\begin{array}{r}-0.5 \\
-0.8 \\
0.0 \\
-1.1 \\
-2.3\end{array}$ \\
\hline $\begin{array}{r}14.0 \\
15.0 \\
16.0 \\
18.0 \\
19.0\end{array}$ & $\begin{array}{l}\text { M. } \\
\text { L. } \\
\text { M. } \\
\text { P. } \\
\text { M. }\end{array}$ & & . & $\begin{array}{lll}23 & 35 & 13.85 \\
23 & 38 & 53.76 \\
23 & 42 & 33.19 \\
23 & 49 & 51.51 \\
23 & 53 & 30.28\end{array}$ & $\begin{array}{r}-0.17 \\
0.00 \\
-0.04 \\
+0.04 \\
-0.02\end{array}$ & $\begin{array}{l}64.80 \\
64.70 \\
64.52 \\
64.58 \\
64.56\end{array}$ & $\begin{array}{l}+0.11 \\
+0.05 \\
+0.09 \\
+0.03 \\
+0.03\end{array}$ & $:$ & $\begin{array}{rrr}-2 & 40 & 44.2 \\
-2 & 17 & 3.2 \\
-\because & \because 5 & 36.4 \\
-0 & 42 & 15.1\end{array}$ & $\begin{array}{l}+0.4 \\
+1.2 \\
+1.8 \\
+0.5\end{array}$ & $\begin{array}{cc}16 & 6.4 \\
16 & 6.6 \\
10 & \cdots . .8 \\
16 & 5.1 \\
16 & 4.1\end{array}$ & $\begin{array}{l}-0.4 \\
+0.1 \\
+0.1 \\
-1.3\end{array}$ \\
\hline $\begin{array}{l}21.0 \\
22.0 \\
23.0 \\
24.0 \\
25.0\end{array}$ & $\begin{array}{l}\text { M. } \\
\text { P. } \\
\text { L. } \\
\text { M. }\end{array}$ & E. & : & $\begin{array}{rrr}0 & 0 & 47.46 \\
0 & 4 & 25.85 \\
0 & 8 & 4.08 \\
0 & 11 & 42.14 \\
0 & 15 & 20.36\end{array}$ & $\begin{array}{l}+0.04 \\
+0.07 \\
+0.04 \\
-0.06 \\
+0.05\end{array}$ & $\begin{array}{l}64.50 \\
64.50 \\
64.48 \\
64.43 \\
64.40\end{array}$ & $\begin{array}{l}+0.01 \\
+0.02 \\
+0.01 \\
+0.03 \\
-0.05\end{array}$ & : & $\begin{array}{rrr}+ & 5 & 5.3 \\
+ & 028 & 50.1 \\
+ & 05229.1 \\
+1168.0 & 16 \\
+1 & 3943.3\end{array}$ & $\begin{array}{l}+0.5 \\
+0.6 \\
+0.3 \\
+1.4 \\
+0.8\end{array}$ & $\begin{array}{ll}16 & 3.5 \\
16 & 3.8 \\
16 & 4.7 \\
16 & 3.1 \\
16 & 2.6\end{array}$ & $\begin{array}{l}-1.4 \\
-0.8 \\
+0.3 \\
-1.0 \\
-1.2\end{array}$ \\
\hline
\end{tabular}


SUN.

[No correction for personal equation, see pages A cxuvi and A cxurx, nor for equinox, see page A curv, has been applied.]

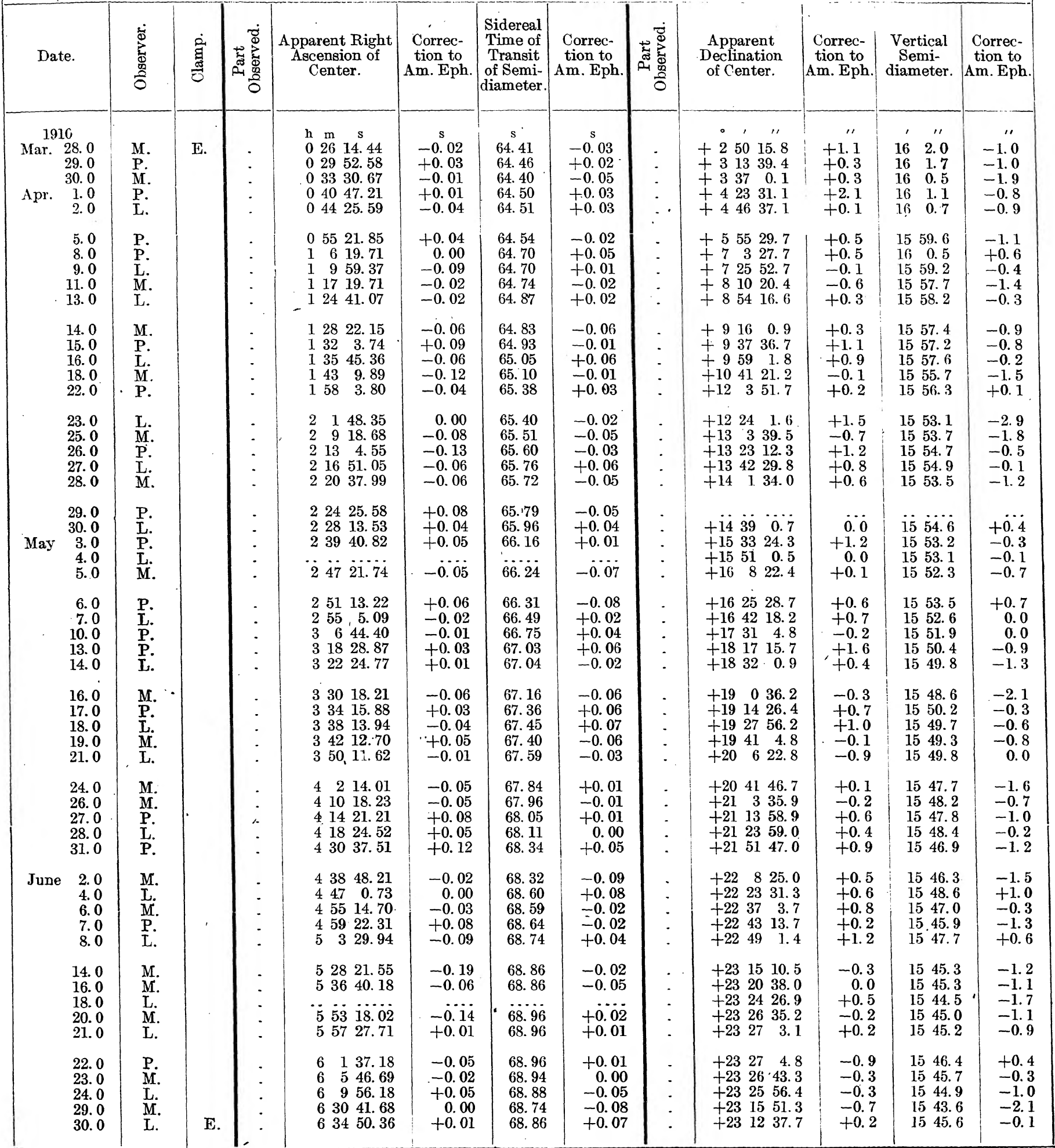




\begin{tabular}{|c|c|c|c|c|c|c|c|c|c|c|c|c|c|}
\hline \multicolumn{14}{|c|}{ SUNT. } \\
\hline \multicolumn{14}{|c|}{ [No correction for personal equation, see pages A CXLVr and A cxulx, nor for equinox, see page A CLIV, has been applied.] } \\
\hline Dat & & 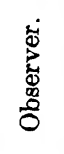 & $\underset{\dot{g}}{\dot{\sigma}}$ & 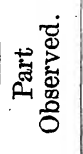 & $\begin{array}{l}\text { Apparent Right } \\
\text { Ascension of } \\
\text { Center. }\end{array}$ & $\begin{array}{c}\text { Correc- } \\
\text { tion to } \\
\text { Am. Eph. }\end{array}$ & $\begin{array}{c}\text { Sidereal } \\
\text { Time of } \\
\text { Transit } \\
\text { of Semi- } \\
\text { diameter. }\end{array}$ & $\begin{array}{c}\text { Correc- } \\
\text { tion to } \\
\text { Am. Eph. }\end{array}$ & . & $\begin{array}{l}\text { Apparent } \\
\text { Declination } \\
\text { of Center. }\end{array}$ & $\begin{array}{c}\text { Correc- } \\
\text { tion to } \\
\text { Am. Eph. }\end{array}$ & $\begin{array}{c}\text { Vertical } \\
\text { Semi- } \\
\text { diameter. }\end{array}$ & $\begin{array}{l}\text { Correc- } \\
\text { tion to } \\
\text { Am. Eph. }\end{array}$ \\
\hline July & $\begin{array}{l}10 \\
1.0 \\
2.0 \\
6.0 \\
8.0 \\
9.0\end{array}$ & $\begin{array}{l}\text { M. } \\
\text { L. } \\
\text { L. } \\
\text { L. } \\
\text { M. }\end{array}$ & E. & $\dot{.}$ & 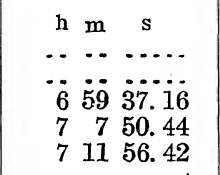 & $\begin{array}{c}s \\
, \ldots . \\
-0.13 \\
+0.01 \\
0.00\end{array}$ & $\begin{array}{c}\text { s } \\
\cdots \ldots \\
68.74 \\
68.44 \\
68.38\end{array}$ & $\begin{array}{c}s \\
\cdots \cdots \\
+0.18 \\
-0.02 \\
-0.02\end{array}$ & $:$ & $\begin{array}{rrr}\circ & \prime & \prime \prime \\
+23 & 8 & 58.2 \\
+23 & 4 & 55.1 \\
+22 & 44 & 40.4 \\
+22 & 32 & 11.2 \\
+22 & 25 & 20.8\end{array}$ & $\begin{array}{r} \\
-0.4 \\
-0.3 \\
-0.5 \\
+0.4 \\
+0.3\end{array}$ & $\begin{array}{rr} & \prime \prime \\
15 & 44.5 \\
15 & 45.3 \\
15 & 46.3 \\
15 & 45.0 \\
15 & 44.8\end{array}$ & $\begin{array}{r}11 \\
-1.2 \\
-0.4 \\
+0.6 \\
-0.7 \\
-0.9\end{array}$ \\
\hline & $\begin{array}{l}11.0 \\
12.0 \\
14.0 \\
15.0 \\
20.0\end{array}$ & $\begin{array}{l}\mathrm{L} . \\
\mathrm{M} . \\
\mathrm{M} \\
\mathrm{L} \\
\mathrm{M}\end{array}$ & ' & $\begin{array}{l}\dot{.} \\
\dot{.} \\
\dot{.}\end{array}$ & $\begin{array}{rrr}7 & 20 & 7.14 \\
7 & 24 & 1.1 .82 \\
7 & 32 & 19.70 \\
7 & 36 & 23.02 \\
7 & 56 & 31.12\end{array}$ & $\begin{array}{r}+0.01 \\
0.00 \\
-0.05 \\
+0.05 \\
-0.03\end{array}$ & $\begin{array}{l}68.28 \\
68.26 \\
68.01 \\
67.98 \\
67.66\end{array}$ & $\begin{array}{r}0.00 \\
+0.04 \\
-0.08 \\
-0.04 \\
+0.01\end{array}$ & \begin{tabular}{l|}
$:$ \\
$:$ \\
$:$ \\
$:$
\end{tabular} & $\begin{array}{rrr}+22 & 10 & 30.3 \\
+22 & 230.6 \\
+21 & 4523.7 \\
+21 & 36 & 17.2 \\
+20 & 4516.1\end{array}$ & $\begin{array}{r}0.0 \\
-0.2 \\
-0.2 \\
+0.3 \\
+0.9\end{array}$ & $\begin{array}{ll}15 & 45.9 \\
15 & 44.8 \\
15 & 44.0 \\
15 & 43.9 \\
15 & 45.0\end{array}$ & $\begin{array}{l}+0.1 \\
-1.0 \\
-1.9 \\
-2.1 \\
-1.3\end{array}$ \\
\hline & $\begin{array}{l}21.0 \\
22.0 \\
25.0 \\
26.0 \\
28.0\end{array}$ & $\begin{array}{l}\mathrm{P} . \\
\mathrm{M} \\
\mathrm{P} \\
\mathrm{M}\end{array}$ & & $\dot{.}$ & $\begin{array}{rrr}8 & 0 & 31.16 \\
8 & 4 & 30.49 \\
8 & 16 & 25.46 \\
8 & 20 & 22.51 \\
8 & 28 & 15.06\end{array}$ & $\begin{array}{l}+0.02 \\
-0.08 \\
+0.04 \\
-0.05 \\
-0.05\end{array}$ & $\begin{array}{l}67.60 \\
67.49 \\
67.24 \\
67.11 \\
66.95\end{array}$ & $\begin{array}{r}+0.03 \\
0.00 \\
0.00 \\
-0.05 \\
-0.04\end{array}$ & $\dot{:}$ & $\begin{array}{r}+203359.8 \\
+202222.0 \\
+194531.5 \\
+193234.6 \\
+19541.7\end{array}$ & $\begin{array}{l}+0.5 \\
-0.6 \\
+0.9 \\
+1.1 \\
+0.5\end{array}$ & $\begin{array}{ll}15 & 45.5 \\
15 & 44.2 \\
15 & 45.0 \\
15 & 45.3 \\
15.45 .3\end{array}$ & $\begin{array}{l}-0.9 \\
-2.3 \\
-1.7 \\
-1.5 \\
-1.7\end{array}$ \\
\hline Aug. & $\begin{array}{r}29.0 \\
30.0 \\
1.0 \\
2.0 \\
3.0\end{array}$ & $\begin{array}{l}\mathrm{P} . \\
\mathrm{M} . \\
\mathrm{P} . \\
\mathrm{P} . \\
\mathrm{M} .\end{array}$ & & : & $\begin{array}{lrr}8 & 32 & 10.52 \\
8 & 36 & 5.19 \\
8 & 43 & 53.33 \\
8 & 47 & 46.35 \\
8 & 51 & 38.69\end{array}$ & $\begin{array}{r}0.00 \\
-0.16 \\
+0.09 \\
+0.05 \\
-0.07\end{array}$ & $\begin{array}{l}66.95 \\
66.76 \\
66.68 \\
66.50 \\
66.46\end{array}$ & $\begin{array}{r}+0.04 \\
-0.06 \\
+0.04 \\
-0.05 \\
0.00\end{array}$ & \begin{tabular}{l|}
- \\
$:$ \\
$:$ \\
.
\end{tabular} & $\begin{array}{r}+185148.2 \\
+183733.5 \\
+18812.8 \\
+1753 \\
+173738.2\end{array}$ & $\begin{array}{l}+1.8 \\
+0.7 \\
+1.9 \\
+0.4 \\
+0.7\end{array}$ & $\begin{array}{ll}15 & 46.1 \\
15 & 46.2 \\
15 & 46.4 \\
15 & 46.6 \\
15 & 46.1\end{array}$ & $\begin{array}{l}-1.0 \\
-1.0 \\
-1.0 \\
-1.0 \\
-1.6\end{array}$ \\
\hline & $\begin{array}{r}4.0 \\
5.0 \\
6.0 \\
9.0 \\
10.0\end{array}$ & $\begin{array}{l}\text { P. } \\
\text { M. } \\
\text { L. } \\
\text { L. } \\
\text { P. }\end{array}$ & & $\begin{array}{l}. \\
\dot{.} \\
\dot{.}\end{array}$ & $\begin{array}{rrr}8 & 55 & 30.68 \\
8 & 59 & 21.89 \\
9 & 3 & 12.53 \\
9 & 14 & 40.80 \\
\ldots & \ldots & \cdots\end{array}$ & $\begin{array}{r}+0.06 \\
+0.02 \\
+0.01 \\
-0.07 \\
\ldots \ldots\end{array}$ & $\begin{array}{l}66.42 \\
66.27 \\
66.28 \\
66.06 \\
\cdots \cdots\end{array}$ & $\begin{array}{r}+0.05 \\
-0.02 \\
+0.08 \\
+0.11 \\
\ldots\end{array}$ & $\dot{-}$ & $\begin{array}{r}+172155.1 \\
+17555.0 \\
+164939.6 \\
+155914.2 \\
+154154.0\end{array}$ & $\begin{array}{l}+0.4 \\
+0.1 \\
+1.0 \\
+1.4 \\
+1.0\end{array}$ & $\begin{array}{ll}15 & 47.1 \\
15 & 46.6 \\
15 & 48.4 \\
15 & 49.0 \\
15 & 47.4\end{array}$ & $\begin{array}{l}-0.7 \\
-1.4 \\
+0.3 \\
+0.5 \\
-1.3\end{array}$ \\
\hline & $\begin{array}{l}11.0 \\
12.0 \\
13.0 \\
18.0 \\
19.0\end{array}$ & $\begin{array}{l}\mathrm{L} . \\
\mathrm{P} . \\
\mathrm{L} . \\
\mathrm{P} . \\
\mathrm{L} .\end{array}$ & & $\begin{array}{l}. \\
. \\
.\end{array}$ & $\begin{array}{llr}9 & 22 & 16.75 \\
9 & 26 & 3.87 \\
9 & 29 & 50.31 \\
9 & 48 & 34.51 \\
9 & 52 & 17.65\end{array}$ & $\begin{array}{l}-0.02 \\
+0.03 \\
-0.01 \\
+0.07 \\
-0.02\end{array}$ & $\begin{array}{l}65.80 \\
65.73 \\
65.55 \\
65.26 \\
65.20\end{array}$ & $\begin{array}{l}+0.02 \\
+0.03 \\
-0.07 \\
+0.02 \\
+0.03\end{array}$ & $\begin{array}{l}: \\
: \\
: \\
\cdot\end{array}$ & $\begin{array}{rr}+15 & 2418.8 \\
+15 & 629.0 \\
+14 & 4824.7 \\
+13 & 1440.6 \\
+125516.9\end{array}$ & $\begin{array}{r}+0.6 \\
+0.3 \\
0.0 \\
+0.8 \\
+0.9\end{array}$ & $\begin{array}{ll}15 & 48.0 \\
15 & 49.7 \\
15 & 47.7 \\
15 & 48.3 \\
15 & 49.8\end{array}$ & $\begin{array}{l}-0.9 \\
+0.7 \\
-1.5 \\
-1.8 \\
-0.5\end{array}$ \\
\hline & $\begin{array}{l}20.0 \\
22.0 \\
23.0 \\
25.0 \\
27.0\end{array}$ & $\begin{array}{l}\text { P. } \\
\text { P. } \\
\text { L. } \\
\text { L. } \\
\text { L. }\end{array}$ & & $\begin{array}{l}. \\
\dot{.} \\
\dot{.} \\
.\end{array}$ & $\begin{array}{rrr}9 & 56 & 0.44 \\
10 & 3 & 24.49 \\
10 & 7 & 5.81 \\
10 & 14 & 27.18 \\
10 & 21 & 47.02\end{array}$ & $\begin{array}{l}+0.04 \\
+0.05 \\
+0.02 \\
-0.03 \\
-0.02\end{array}$ & $\begin{array}{l}65.14 \\
65.00 \\
64.91 \\
64.77 \\
64.77\end{array}$ & $\begin{array}{r}+0.04 \\
+0.04 \\
+0.01 \\
0.00 \\
+0.12\end{array}$ & $\dot{.}$ & $\begin{array}{r}+123540.2 \\
+115553.7 \\
+113542.5 \\
+105447.7 \\
+101310.2\end{array}$ & $\begin{array}{l}+0.2 \\
+1.3 \\
+1.0 \\
+1.1 \\
+0.1\end{array}$ & $\begin{array}{ll}15 & 51.1 \\
15 & 50.5 \\
15 & 51.1 \\
15 & 52.0 \\
15 & 51.9\end{array}$ & $\begin{array}{r}+0.6 \\
-0.4 \\
0.0 \\
+0.5 \\
0.0\end{array}$ \\
\hline Sept. & $\begin{array}{r}30.0 \\
6.0 \\
7.0 \\
8.0 \\
9.0\end{array}$ & $\begin{array}{l}\text { L. } \\
\text { P. } \\
\text { M. } \\
\text { P. } \\
\text { M. }\end{array}$ & & $\begin{array}{l}- \\
.\end{array}$ & 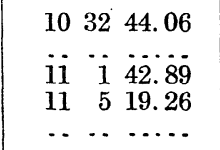 & $\begin{array}{r}-0.03 \\
+0.01 \\
+0.13 \\
\ldots \ldots\end{array}$ & $\begin{array}{l}64.49 \\
64.14 \\
64.19 \\
\ldots \ldots\end{array}$ & $\begin{array}{r}0.00 \\
-0.02 \\
+0.06 \\
\ldots\end{array}$ & $\dot{\dot{.}}$ & $\begin{array}{r}9931.7 \\
+6368.2 \\
+61345.2 \\
+55115.1 \\
+52839.8\end{array}$ & $\begin{array}{l}-0.3 \\
+0.2 \\
+0.8 \\
+0.4 \\
+0.5\end{array}$ & $\begin{array}{ll}15 & 52.3 \\
15 & 53.8 \\
15 & 53.6 \\
15 & 54.5 \\
15 & 53.7\end{array}$ & $\begin{array}{l}-0.2 \\
-0.3 \\
-0.8 \\
-0.1 \\
-1.2\end{array}$ \\
\hline . & $\begin{array}{l}10.0 \\
12.0 \\
13.0 \\
14.0 \\
15.0\end{array}$ & $\begin{array}{l}\mathrm{P} . \\
\mathbf{P} \\
\mathrm{M} \\
\mathrm{P} . \\
\mathrm{M}\end{array}$ & & $\begin{array}{l}. \\
\dot{.} \\
\dot{.}\end{array}$ & $\begin{array}{lll}11 & 12 & 31.13 \\
11 & 19 & 42.44 \\
11 & 23 & 17.81 \\
11 & 26 & 53.28 \\
11 & 30 & 28.53\end{array}$ & $\begin{array}{l}+0.07 \\
+0.10 \\
+0.02 \\
+0.13 \\
+0.10\end{array}$ & $\begin{array}{l}64.13 \\
64.12 \\
63.98 \\
64.00 \\
63.97\end{array}$ & $\begin{array}{l}+0.05 \\
+0.07 \\
-0.06 \\
-0.03 \\
-0.05\end{array}$ & $\begin{array}{l}\dot{.} \\
\dot{.} \\
\dot{.}\end{array}$ & $\begin{array}{r}5559.3 \\
+42022.5 \\
+35727.2 \\
+33427.9 \\
+31124.4\end{array}$ & $\begin{array}{l}+0.9 \\
+0.8 \\
+0.7 \\
+0.7 \\
+0.2\end{array}$ & $\begin{array}{ll}15 & 54.5 \\
15 & 55.0 \\
15 & 55.0 \\
15 & 54.5 \\
15 & 55.2\end{array}$ & $\begin{array}{l}-0.6 \\
-0.6 \\
-0.9 \\
-1.7 \\
-1.2\end{array}$ \\
\hline . & $\begin{array}{l}16.0 \\
17.0 \\
20.0 \\
21.0 \\
22.0\end{array}$ & $\begin{array}{l}\text { P. } \\
\text { L. } \\
\text { L. } \\
\text { M. } \\
\text { L. }\end{array}$ & & $\begin{array}{l}\dot{.} \\
\dot{.} \\
\dot{.}\end{array}$ & 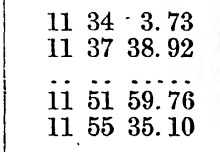 & $\begin{array}{r}+0.08 \\
+0.09 \\
+0.04 \\
0.00\end{array}$ & $\begin{array}{l}64.03 \\
64.00 \\
64.01 \\
64.12\end{array}$ & $\begin{array}{l}+0.02 \\
-0.01 \\
-0.02 \\
+0.08\end{array}$ & $\begin{array}{l}\dot{.} \\
\dot{\cdot} \\
\dot{\cdot}\end{array}$ & 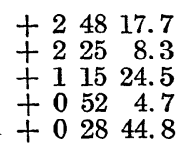 & $\begin{array}{r}-0.1 \\
0.0 \\
+0.9 \\
+0.1 \\
+0.8\end{array}$ & $\begin{array}{ll}15 & 56.6 \\
15 & 56.0 \\
15 & 57.7 \\
15 & 56.8 \\
15 & 58.7\end{array}$ & $\begin{array}{l}-0.1 \\
-1.0 \\
-0.1 \\
-1.2 \\
+0.4\end{array}$ \\
\hline$\cdot$ & $\begin{array}{l}23.0 \\
26.0 \\
27.0 \\
28.0 \\
29.0\end{array}$ & $\begin{array}{l}\text { M. } \\
\mathbf{M} . \\
\mathbf{P} . \\
\text { L. } \\
\text { M. }\end{array}$ & E. & $\begin{array}{l}\dot{.} \\
\dot{.} \\
\dot{.}\end{array}$ & $\begin{array}{rrr}11 & 59 & 10.62 \\
12 & 9 & 57.87 \\
12 & 13 & 34.24 \\
12 & 17 & 10.52 \\
12 & 20 & 47.05\end{array}$ & $\begin{array}{l}+0.03 \\
-0.06 \\
+0.15 \\
+0.06 \\
-0.01\end{array}$ & $\begin{array}{l}63.98 \\
64.18 \\
64.15 \\
64.25 \\
64.28\end{array}$ & $\begin{array}{l}-0.07 \\
+0.05 \\
-0.01 \\
+0.06 \\
+0.06\end{array}$ & $\dot{:} \cdot$ & $\begin{array}{rr}0 & 522.2 \\
-1 & 448.3 \\
-1 & 2812.3 \\
-1 & 5135.2 \\
- & 21456.9\end{array}$ & $\begin{array}{l}+0.2 \\
+0.4 \\
+0.3 \\
+1.0 \\
+2.1\end{array}$ & $\begin{array}{lr}15 & 56.6 \\
15 & 57.9 \\
15 & 57.3 \\
16 & 0.6 \\
16 & 0.7\end{array}$ & $\begin{array}{l}-2.0 \\
-1.4 \\
-2.3 \\
+0.7 \\
+0.6\end{array}$ \\
\hline
\end{tabular}


SUN

[No correction for personal equation, see pages A cxLVI and A CXLIx, nor for equinox, see page A cLrv, has been applied.]

\begin{tabular}{|c|c|c|c|c|c|c|c|c|c|c|c|c|}
\hline Date. & 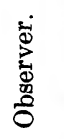 & 苞 & 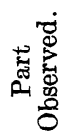 & $\begin{array}{c}\text { Apparent Right } \\
\text { Ascension of } \\
\text { Center. }\end{array}$ & $\begin{array}{c}\text { Correc- } \\
\text { tion to } \\
\text { Am. Eph. }\end{array}$ & $\begin{array}{c}\text { Sidereal } \\
\text { Time of } \\
\text { Transit } \\
\text { of Semi- } \\
\text { diameter. }\end{array}$ & $\begin{array}{c}\text { Correc- } \\
\text { tion to } \\
\text { Am. Eph. }\end{array}$ & 焉总 & $\begin{array}{l}\text { Apparent } \\
\text { Declination } \\
\text { of Center. }\end{array}$ & $\begin{array}{c}\text { Correc- } \\
\text { tion to } \\
\text { Am. Eph. }\end{array}$ & $\begin{array}{c}\text { Vertical } \\
\text { Semi- } \\
\text { diameter. }\end{array}$ & $\begin{array}{c}\text { Correc- } \\
\text { tion to } \\
\text { Am. Eph. }\end{array}$ \\
\hline 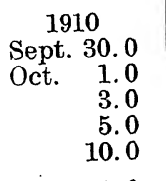 & $\begin{array}{l}\text { P. } \\
\text { L. } \\
\text { M. } \\
\text { M. }\end{array}$ & E. & $\begin{array}{l}\dot{.} \\
\dot{.} \\
\dot{.}\end{array}$ & $\begin{array}{rrr}\mathrm{h} & \mathrm{m} & \mathrm{s} \\
12 & 24 & 24.02 \\
12 & 28 & 1.08 \\
12 & 35 & 16.13 \\
12 & 42 & 32.58 \\
13 & 0 & 50.08\end{array}$ & $\begin{array}{c}\text { s } \\
+0.11 \\
+0.05 \\
-0.02 \\
+0.03 \\
+0.03\end{array}$ & $\begin{array}{l}\mathrm{s} \\
64.30 \\
64.34 \\
64.34 \\
64.47 \\
64.75\end{array}$ & $\begin{array}{c}\text { s } \\
+0.04 \\
+0.04 \\
-0.04 \\
-0.01 \\
-0.04\end{array}$ & $\dot{.}$ & 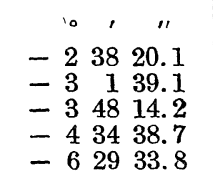 & $\begin{array}{l} \\
+1 \\
+0.6 \\
+1.8 \\
+1.4 \\
+1.0 \\
+0.4\end{array}$ & $\begin{array}{cc}\prime & \prime \prime \\
16 & 0.1 \\
16 & 1.2 \\
16 & 0.0 \\
16 & 1.0 \\
16 & 2.3\end{array}$ & $\begin{array}{l}1 " \\
-0.3 \\
+0.5 \\
-1.2 \\
-0.8 \\
-0.9\end{array}$ \\
\hline $\begin{array}{l}11.0 \\
12.0 \\
13.0 \\
14.0 \\
15.0\end{array}$ & $\begin{array}{l}\mathrm{P} . \\
\mathrm{L} . \\
\mathrm{M} . \\
\mathrm{P} . \\
\mathrm{M} .\end{array}$ & 1 & $\begin{array}{l}- \\
- \\
.\end{array}$ & $\begin{array}{rrr}13 & 4 & 30.92 \\
13 & 8 & 12.07 \\
13 & 11 & 53.78 \\
13 & 15 & 36.01 \\
13 & 19 & 18.69\end{array}$ & $\begin{array}{l}+0.10 \\
+0.02 \\
+0.02 \\
+0.04 \\
-0.02\end{array}$ & $\begin{array}{l}64.90 \\
65.03 \\
65.08 \\
65.09 \\
65.18\end{array}$ & $\begin{array}{r}+0.04 \\
+0.10 \\
+0.07 \\
0.00 \\
+0.01\end{array}$ & $\dot{.}$ & $\begin{array}{r}65219.2 \\
-71456.0 \\
-73730.8 \\
-75956.3 \\
-82216.6\end{array}$ & $\begin{array}{l}-0.5 \\
+1.6 \\
-0.4 \\
+0.6 \\
+0.1\end{array}$ & $\begin{array}{ll}16 & 4.2 \\
16 & 3.6 \\
16 & 4.8 \\
16 & 3.4 \\
16 & 3.0\end{array}$ & $\begin{array}{l}+0.7 \\
-0.2 \\
+0.7 \\
-0.9 \\
-1.6\end{array}$ \\
\hline $\begin{array}{l}17.0 \\
18.0 \\
21.0 \\
22.0 \\
24.0\end{array}$ & $\begin{array}{l}\text { M. } \\
\text { P. } \\
\text { P. } \\
\text { L. } \\
\text { M. }\end{array}$ & & $\begin{array}{l}. \\
\dot{.} \\
.\end{array}$ & $\begin{array}{lll}13 & 26 & 45.78 \\
13 & 30 & 30.35 \\
13 & 41 & 47.31 \\
13 & 45 & 34.29 \\
13 & 53 & 10.18\end{array}$ & $\begin{array}{l}-0.04 \\
+0.11 \\
+0.09 \\
+0.09 \\
-0.04\end{array}$ & $\begin{array}{l}65.31 \\
65.52 \\
65.69 \\
65.82 \\
65.94\end{array}$ & $\begin{array}{r}-0.02 \\
+0.10 \\
0.00 \\
+0.03 \\
-0.05\end{array}$ & $\begin{array}{l}. \\
\dot{.} \\
\dot{.}\end{array}$ & $\begin{array}{rrr}-9 & 633.0 \\
-928 & 30.8 \\
-10 & 33 & 31.8 \\
-10 & 54 & 53.5 \\
-11 & 37 & 8.3\end{array}$ & $\begin{array}{r}+1.2 \\
+0.5 \\
0.0 \\
+0.5 \\
+0.9\end{array}$ & $\begin{array}{ll}16 & 4.0 \\
16 & 5.1 \\
16 & 4.7 \\
16 & 6.0 \\
16 & 5.7\end{array}$ & $\begin{array}{l}-1.2 \\
-0.3 \\
-1.5 \\
-0.5 \\
-1.3\end{array}$ \\
\hline $\begin{array}{r}26.0 \\
28.0 \\
31.0 \\
\text { Nov. } \begin{array}{r}1.0 \\
5.0\end{array}\end{array}$ & $\begin{array}{l}\mathrm{L} . \\
\mathrm{P} \\
\mathrm{M} . \\
\mathrm{P} . \\
\mathrm{L} .\end{array}$ & & $\dot{.}$ & $\begin{array}{rrr}14 & 0 & 49.21 \\
14 & 8 & 31.09 \\
14 & 20 & 9.61 \\
14 & 24 & 4.18 \\
14 & 39 & 49.87\end{array}$ & $\begin{array}{l}+0.08 \\
+0.08 \\
-0.01 \\
+0.13 \\
+0.01\end{array}$ & $\begin{array}{l}66.29 \\
66.52 \\
66.71 \\
66.88 \\
67.32\end{array}$ & $\begin{array}{r}+0.09 \\
+0.11 \\
-0.03 \\
+0.03 \\
0.00\end{array}$ & $\begin{array}{l}- \\
- \\
- \\
-\end{array}$ & $\begin{array}{rrr}-12 & 18 & 42.0 \\
-12 & 59 & 29.7 \\
-13 & 59 & 12.0 \\
-14 & 18 & 38.7 \\
-15 & 34 & 6.5\end{array}$ & $\begin{array}{l}+0.3 \\
+1.1 \\
-0.5 \\
+0.4 \\
+0.6\end{array}$ & $\begin{array}{ll}16 & 7.8 \\
16 & 8.3 \\
16 & 7.3 \\
16 & 7.6 \\
16 & 9.9\end{array}$ & $\begin{array}{l}+0.3 \\
+0.3 \\
-1.5 \\
-1.4 \\
-0.1\end{array}$ \\
\hline $\begin{array}{r}7.0 \\
8.0 \\
9.0 \\
11.0 \\
12.0\end{array}$ & $\begin{array}{l}\text { M. } \\
\text { P. } \\
\text { L. } \\
\text { P. }\end{array}$ & & $\begin{array}{l}. \\
. \\
.\end{array}$ & $\begin{array}{rrr}14 & 51 & 47.90 \\
14 & 55 & 48.77 \\
15 & 3 & 53.17 \\
15 & 7 & 56.71\end{array}$ & $\begin{array}{l}+0.11 \\
+0.02 \\
+0.01 \\
+0.09\end{array}$ & $\begin{array}{l}67.71 \\
67.88 \\
68.06 \\
68.22\end{array}$ & $\begin{array}{r}+0.03 \\
+0.08 \\
+0.02 \\
+0.06\end{array}$ & $\begin{array}{l}\cdot \\
\cdot \\
\cdot \\
\cdot\end{array}$ & $\begin{array}{rrr}-16 & 10 & 19.5 \\
-16 & 28 & 1.0 \\
-16 & 45 & 25.5 \\
-17 & 19 & 25.8 \\
-17 & 35 & 58.4\end{array}$ & $\begin{array}{r}+0.3 \\
+0.8 \\
+1.6 \\
0.0 \\
0.0\end{array}$ & $\begin{array}{ll}16 & 10.0 \\
16 & 10.1 \\
16 & 11.1 \\
16 & 11.5 \\
16 & 12.9\end{array}$ & $\begin{array}{r}-0.5 \\
-0.7 \\
+0.1 \\
0.0 \\
+1.2\end{array}$ \\
\hline $\begin{array}{l}14.0 \\
15.0 \\
17.0 \\
18.0 \\
19.0\end{array}$ & $\begin{array}{l}\mathrm{M} . \\
\mathrm{P} . \\
\mathrm{M} . \\
\mathrm{P}\end{array}$ & & $\begin{array}{l}\dot{.} \\
\dot{.} \\
\dot{.}\end{array}$ & $\begin{array}{ccc}15 & 16 & 5.96 \\
15 & 28 & 26.47 \\
15 & 32 & 35.01 \\
15 & 36 & 44.29\end{array}$ & $\begin{array}{r}-0.07 \\
+0.03 \\
+0.08 \\
+0.03\end{array}$ & $\begin{array}{l}68.39 \\
68.72 \\
68.90 \\
68.94\end{array}$ & $\begin{array}{r}-0.01 \\
-0.03 \\
+0.03 \\
-0.04\end{array}$ & $\begin{array}{l}\dot{-} \\
\dot{\cdot} \\
\dot{\cdot}\end{array}$ & $\begin{array}{lrr}-18 & 8 & 7.7 \\
-18 & 23 & 43.7 \\
-18 & 53 & 59.1 \\
-19 & 8 & 36.1 \\
-19 & 22 & 51.8\end{array}$ & $\begin{array}{l}+0.6 \\
+1.1 \\
+0.1 \\
+0.2 \\
+1.1\end{array}$ & $\begin{array}{ll}16 & 11.1 \\
16 & 10.9 \\
16 & 11.1 \\
16 & 12.9 \\
16 & 12.1\end{array}$ & $\begin{array}{l}-1.1 \\
-1.5 \\
-1.7 \\
-0.1 \\
-1.1\end{array}$ \\
\hline $\begin{array}{r}21.0 \\
23.0 \\
26.0 \\
\text { Dec. } \begin{array}{r}2.0 \\
3.0\end{array}\end{array}$ & $\begin{array}{l}\text { M. } \\
\mathrm{L} . \\
\mathrm{L} . \\
\mathrm{P} . \\
\mathrm{L} .\end{array}$ & . & $\begin{array}{l}- \\
- \\
- \\
-\end{array}$ & \begin{tabular}{|rrr}
15 & 45 & 5.41 \\
15 & 53 & 30.00 \\
16 & 6 & 12.59 \\
16 & 31 & 58.14 \\
16 & 36 & 18.08
\end{tabular} & $\begin{array}{r}-0.03 \\
+0.08 \\
-0.06 \\
0.00 \\
+0.01\end{array}$ & $\begin{array}{l}69.19 \\
69.54 \\
69.76 \\
70.30 \\
70.44\end{array}$ & $\begin{array}{r}-0.01 \\
+0.12 \\
+0.03 \\
0.00 \\
+0.05\end{array}$ & $\begin{array}{l}. \\
- \\
- \\
\cdot\end{array}$ & $\begin{array}{rrr}-19 & 50 & 21.8 \\
-20 & 16 & 25.2 \\
-20 & 52 & 43.7 \\
-21 & 54 & 37.9 \\
-22 & .3 & 30.0\end{array}$ & $\begin{array}{l}+1.1 \\
+1.6 \\
+0.4 \\
+1.0 \\
+1.2\end{array}$ & $\begin{array}{ll}16 & 12.6 \\
16 & 13.8 \\
16 & 14.8 \\
16 & 14.9 \\
16 & 15.4\end{array}$ & $\begin{array}{l}-1.0 \\
-0.1 \\
+0.3 \\
-0.5 \\
-0.2\end{array}$ \\
\hline $\begin{array}{r}7.0 \\
8.0 \\
9.0 \\
10.0 \\
12.0\end{array}$ & $\begin{array}{l}\mathrm{L} . \\
\mathrm{M} . \\
\mathrm{P} . \\
\mathrm{L} . \\
\mathrm{P} .\end{array}$ & & $\begin{array}{l}- \\
- \\
- \\
-\end{array}$ & $\begin{array}{rrr}16 & 53 & 43.65 \\
16 & 58 & 6.24 \\
17 & 2 & 29.40 \\
17 & 6 & 53.01 \\
17 & 15 & 41.23\end{array}$ & $\begin{array}{l}+0.11 \\
+0.03 \\
+0.06 \\
+0.11 \\
+0.03\end{array}$ & $\begin{array}{l}70.68 \\
70.73 \\
70.79 \\
70.84 \\
71.09\end{array}$ & $\begin{array}{r}0.00 \\
-0.02 \\
-0.03 \\
-0.04 \\
+0.10\end{array}$ & $\begin{array}{l}. \\
. \\
. \\
.\end{array}$ & $\begin{array}{lrr}-22 & 34 & 41.8 \\
-22 & 41 & 23.2 \\
-22 & 47 & 38.8 \\
-22 & 53 & 25.9 \\
-23 & .3 & 40.5\end{array}$ & $\begin{array}{l}+0.2 \\
+0.7 \\
+0.1 \\
+1.0 \\
+0.9\end{array}$ & $\begin{array}{ll}16 & 15.8 \\
16 & 14.6 \\
16 & 14.7 \\
16 & 15.8 \\
16 & 15.1\end{array}$ & $\begin{array}{l}-0.3 \\
-1.7 \\
-1.7 \\
-0.7 \\
-1.7\end{array}$ \\
\hline $\begin{array}{l}13.0 \\
15.0 \\
16.0 \\
17.0 \\
21.0\end{array}$ & $\begin{array}{l}\text { M. } \\
\text { M. } \\
\text { P. } \\
\text { L. } \\
\text { L. }\end{array}$ & . & i. & $\begin{array}{lll}\ddot{17} & \ddot{28} & \mathbf{5 6} .13 \\
17 & 33 & 21.74 \\
17 & 37 & 47.48 \\
17 & 55 & 32.53\end{array}$ & $\begin{array}{r}-0.05 \\
+0.01 \\
-0.05 \\
+0.05\end{array}$ & $\begin{array}{l}71.13 \\
71.15 \\
71.19 \\
71.25\end{array}$ & $\begin{array}{r}+0.01 \\
0.00 \\
+0.01 \\
0.00\end{array}$ & $\dot{:}$ & $\begin{array}{lrr}-23 & 8 & 6.2 \\
-23 & 15 & 35.6 \\
-23 & 18 & 39.1 \\
-23 & 21 & 13.5 \\
-23 & 26 & 52.5\end{array}$ & $\begin{array}{l}+1.3 \\
+1.3 \\
+0.7 \\
+1.3 \\
+1.5\end{array}$ & $\begin{array}{ll}16 & 15.4 \\
16 & 16.1 \\
16 & 16.8 \\
16 & 18.7 \\
16 & 16.8\end{array}$ & $\begin{array}{l}-1.5 \\
-1.0 \\
-0.3 \\
+1.5 \\
-0.7\end{array}$ \\
\hline $\begin{array}{r}22.0 \\
27.0 \\
29.0 \\
31.0 \\
1911\end{array}$ & $\begin{array}{l}\mathrm{M} . \\
\mathrm{P} . \\
\mathrm{M} \\
\mathrm{P} .\end{array}$ & & $\dot{.}$ & $\begin{array}{rrr}17 & 59 & 59.01 \\
18 & 22 & 11.83 \\
18 & 31 & 4.10 \\
18 & 39 & 55.93\end{array}$ & $\begin{array}{l}-0.01 \\
+0.07 \\
-0.10 \\
+0.07\end{array}$ & $\begin{array}{l}71.31 \\
71.24 \\
71.12 \\
71.08\end{array}$ & $\begin{array}{r}+0.05 \\
+0.01 \\
-0.06 \\
-0.02\end{array}$ & $\dot{.}$ & $\begin{array}{rrr}-23 & 27 & 7.4 \\
-23 & 21 & 13.3 \\
-23 & 15 & 36.2 \\
-23 & 8 & 2.8\end{array}$ & $\begin{array}{l}+0.9 \\
+2.5 \\
+0.8 \\
+3.5\end{array}$ & $\begin{array}{ll}16 & 18.0 \\
16 & 17.8 \\
16 & 15.8 \\
16 & 17.0\end{array}$ & $\begin{array}{l}+0.5 \\
+0.1 \\
-2.0 \\
-0.8\end{array}$ \\
\hline $\begin{array}{r}\text { Jan. } \quad 4.0 \\
5.0 \\
6.0 \\
7.0 \\
10.0\end{array}$ & $\begin{array}{l}\mathrm{L} . \\
\mathrm{M} . \\
\mathrm{P} . \\
\mathrm{L} . \\
\mathrm{P} .\end{array}$ & & : & $\begin{array}{rrr}18 & 57 & 35.58 \\
19 & 1 & 59.49 \\
19 & 6 & 23.25 \\
19 & 10 & 46.20 \\
19 & 23 & 52.43\end{array}$ & $\begin{array}{l}-0.02 \\
-0.10 \\
+0.12 \\
+0.01 \\
+0.15\end{array}$ & $\begin{array}{l}70.98 \\
70.92 \\
70.85 \\
70.78 \\
70.54\end{array}$ & $\begin{array}{r}+0.05 \\
+0.05 \\
+0.04 \\
+0.03 \\
0.00\end{array}$ & $\begin{array}{l}\dot{.} \\
\dot{.} \\
\dot{.}\end{array}$ & $\begin{array}{rrr}-22 & 47 & 30.9 \\
-22 & 41 & 14.6 \\
-22 & 34 & 30.5 \\
-22 & 27 & 19.6 \\
-22 & 3 & 9.7\end{array}$ & $\begin{array}{l}+1.5 \\
+0.9 \\
+1.1 \\
+1.3 \\
+0.6\end{array}$ & $\begin{array}{ll}16 & 18.0 \\
16 & 16.6 \\
16 & 17.0 \\
16 & 16.7 \\
16 & 18.5\end{array}$ & $\begin{array}{l}+0.2 \\
-1.2 \\
-0.8 \\
-1.1 \\
+0.8\end{array}$ \\
\hline $\begin{array}{l}16.0 \\
18.0 \\
19.0 \\
20.0 \\
23.0\end{array}$ & $\begin{array}{l}\text { M. } \\
\text { L. } \\
\text { M. } \\
\text { P. } \\
\text { M. }\end{array}$ & E. & : & $\begin{array}{rrr}19 & 49 & 48.25 \\
19 & 58 & 21.85 \\
20 & 2 & 37.45 \\
20 & 6 & 52.46 \\
20 & 19 & 32.90\end{array}$ & $\begin{array}{l}-0.09 \\
+0.06 \\
-0.02 \\
+0.03 \\
+0.02\end{array}$ & $\begin{array}{l}70.07 \\
69.85 \\
69.86 \\
69.64 \\
69.39\end{array}$ & $\begin{array}{r}+0.04 \\
+0.01 \\
+0.12 \\
0.00 \\
+0.07\end{array}$ & $\begin{array}{l}. \\
. \\
. \\
.\end{array}$ & $\begin{array}{rrr}-21 & 3 & 19.5 \\
-20 & 40 & 5.2 \\
-20 & 27 & 54.7 \\
-20 & 15 & 19.4 \\
-19 & 35 & 20.4\end{array}$ & $\begin{array}{l}+0.6 \\
+2.0 \\
+0.6 \\
+0.9 \\
-0.9\end{array}$ & $\begin{array}{ll}16 & 16.1 \\
16 & 17.0 \\
16 & 16.3 \\
16 & 15.9 \\
16 & 15.7\end{array}$ & $\begin{array}{l}-1.3 \\
-0.2 \\
-0.9 \\
-1.2 \\
-1.1\end{array}$ \\
\hline
\end{tabular}




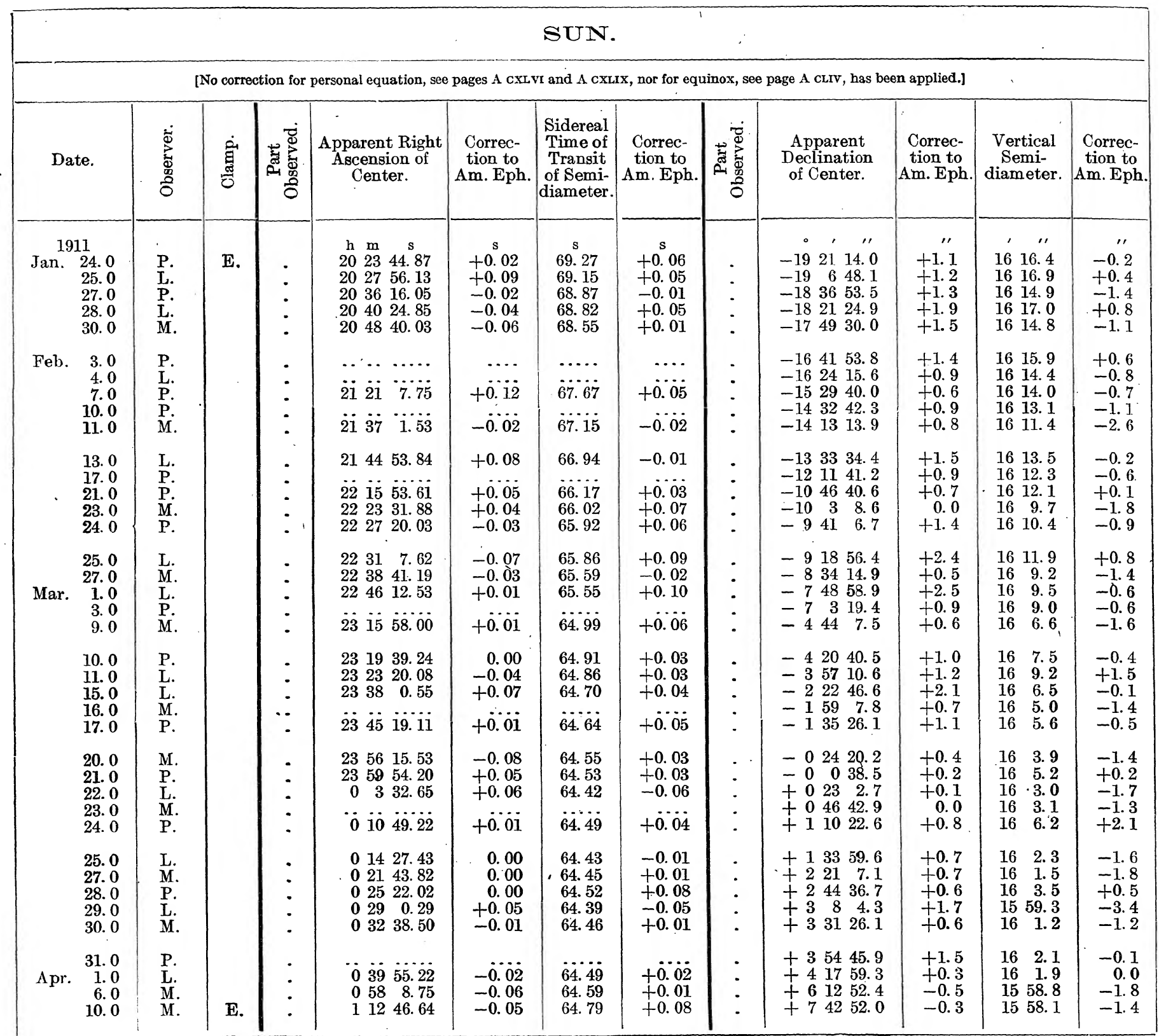




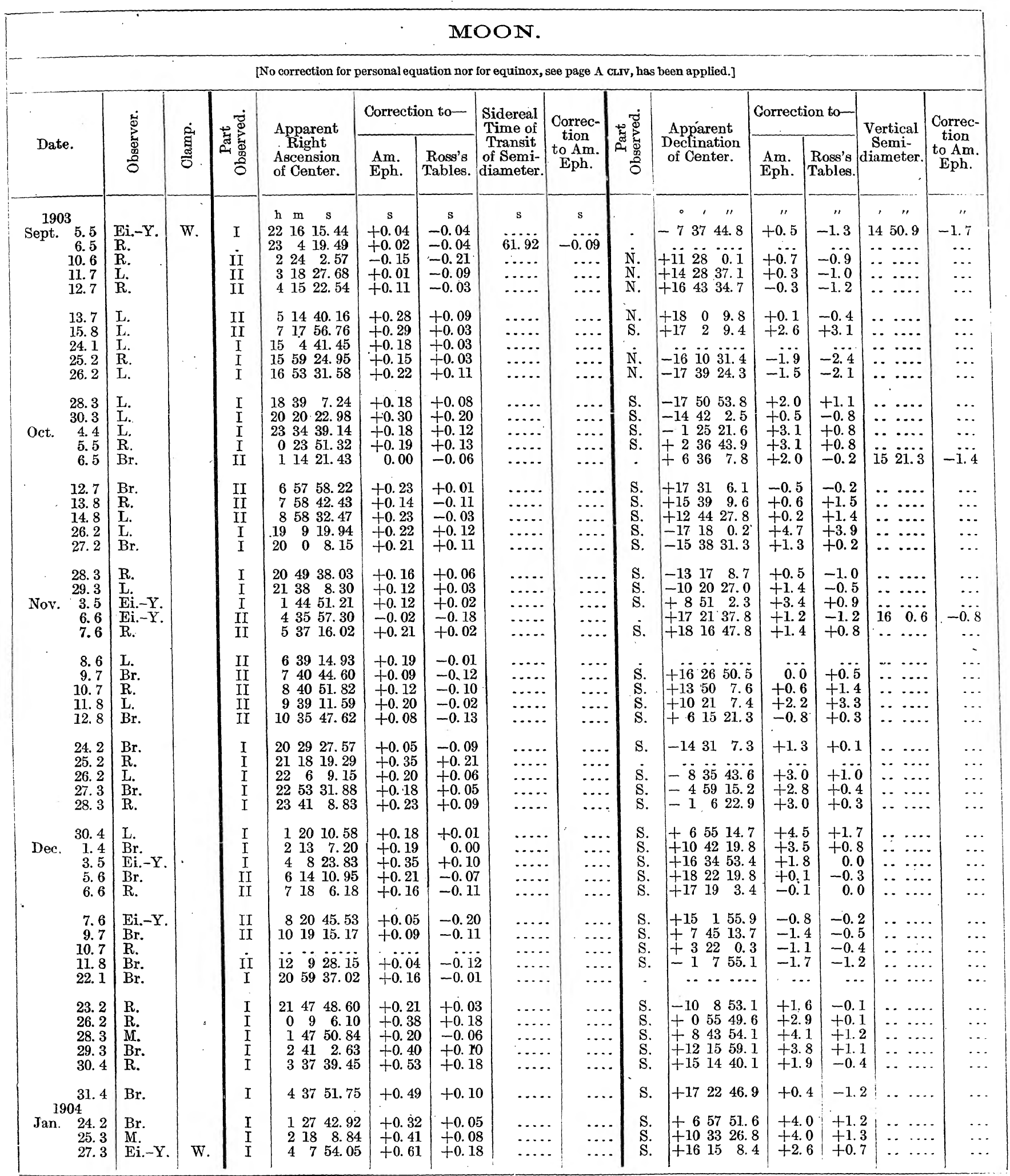




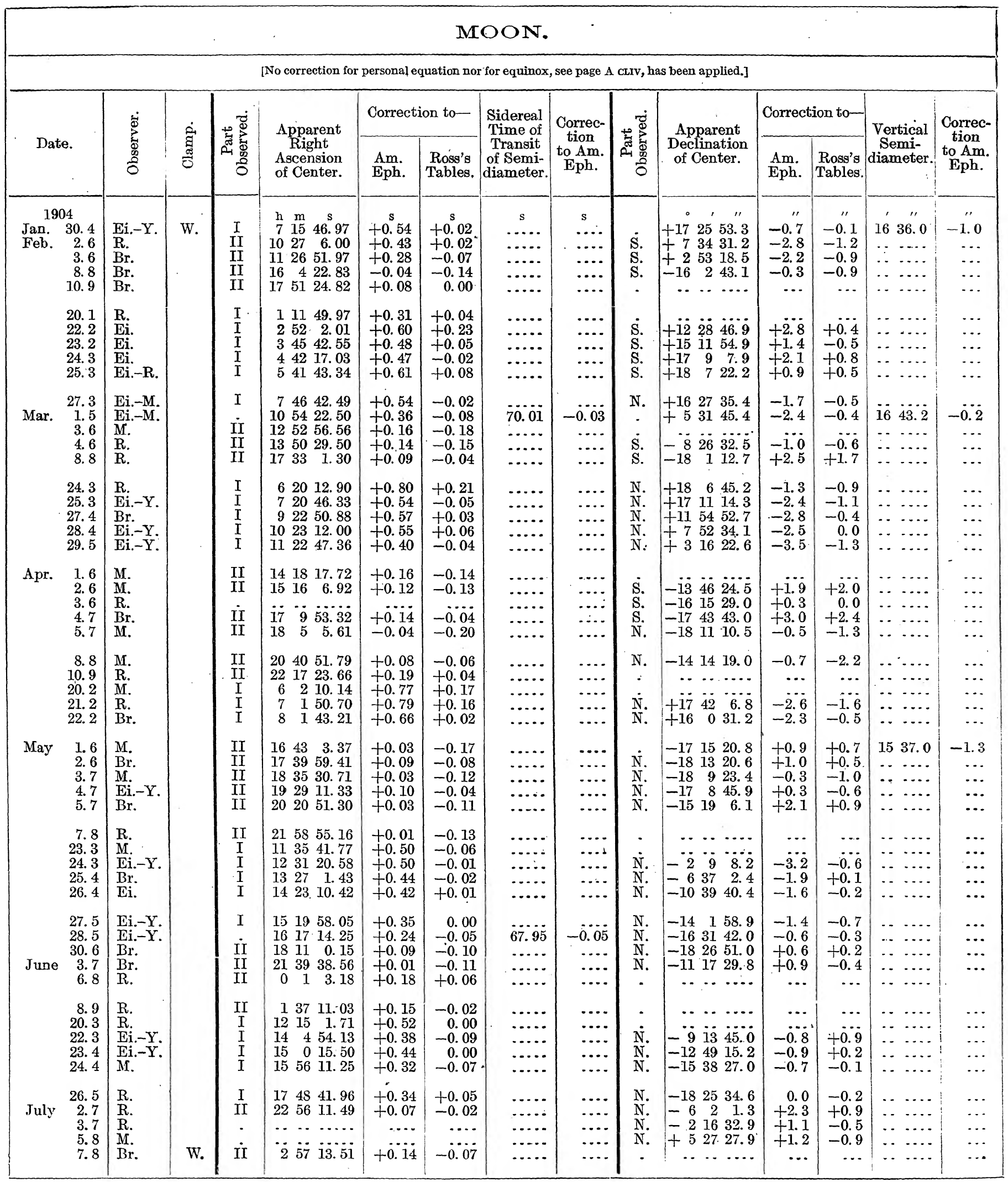


बু

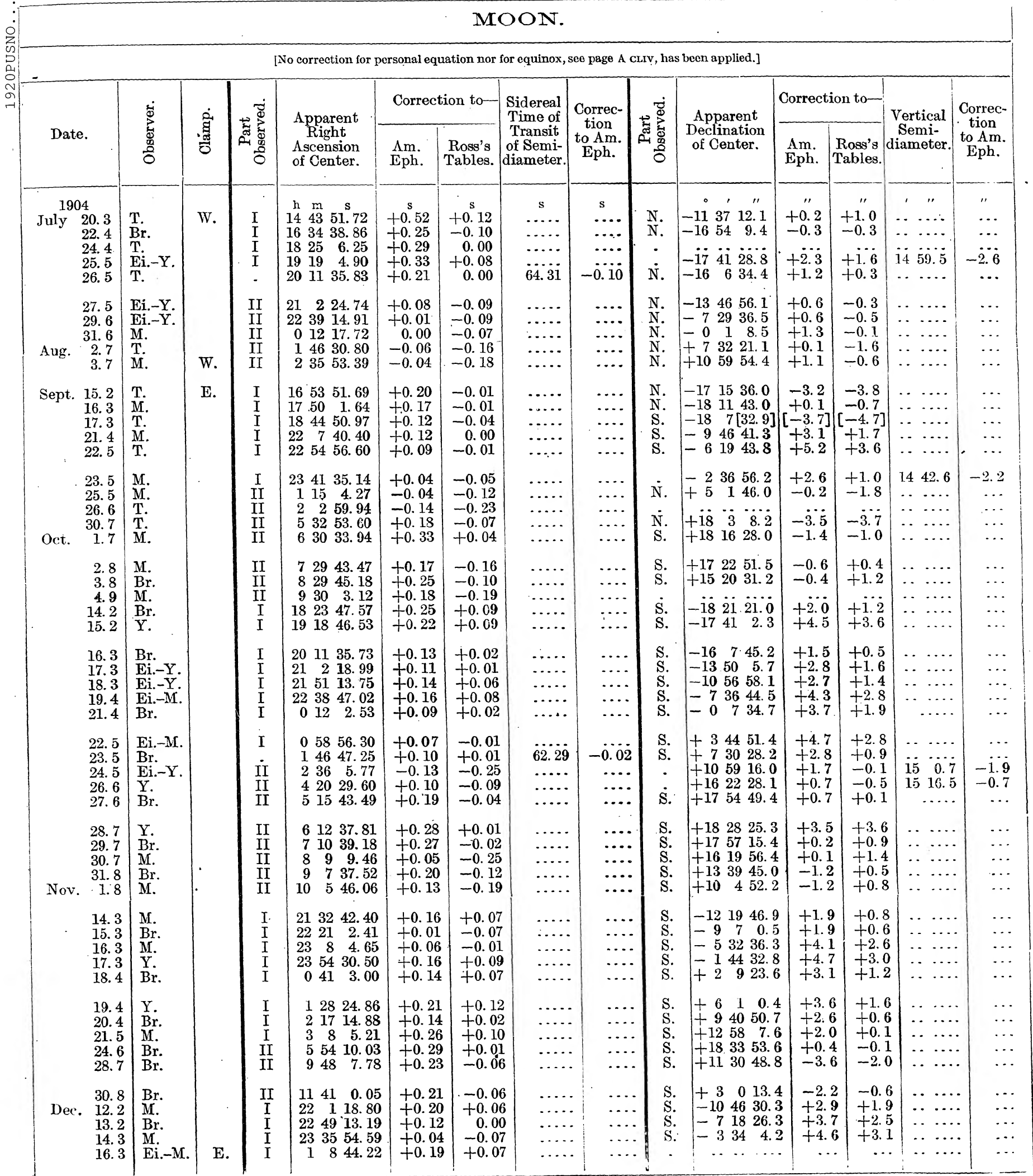




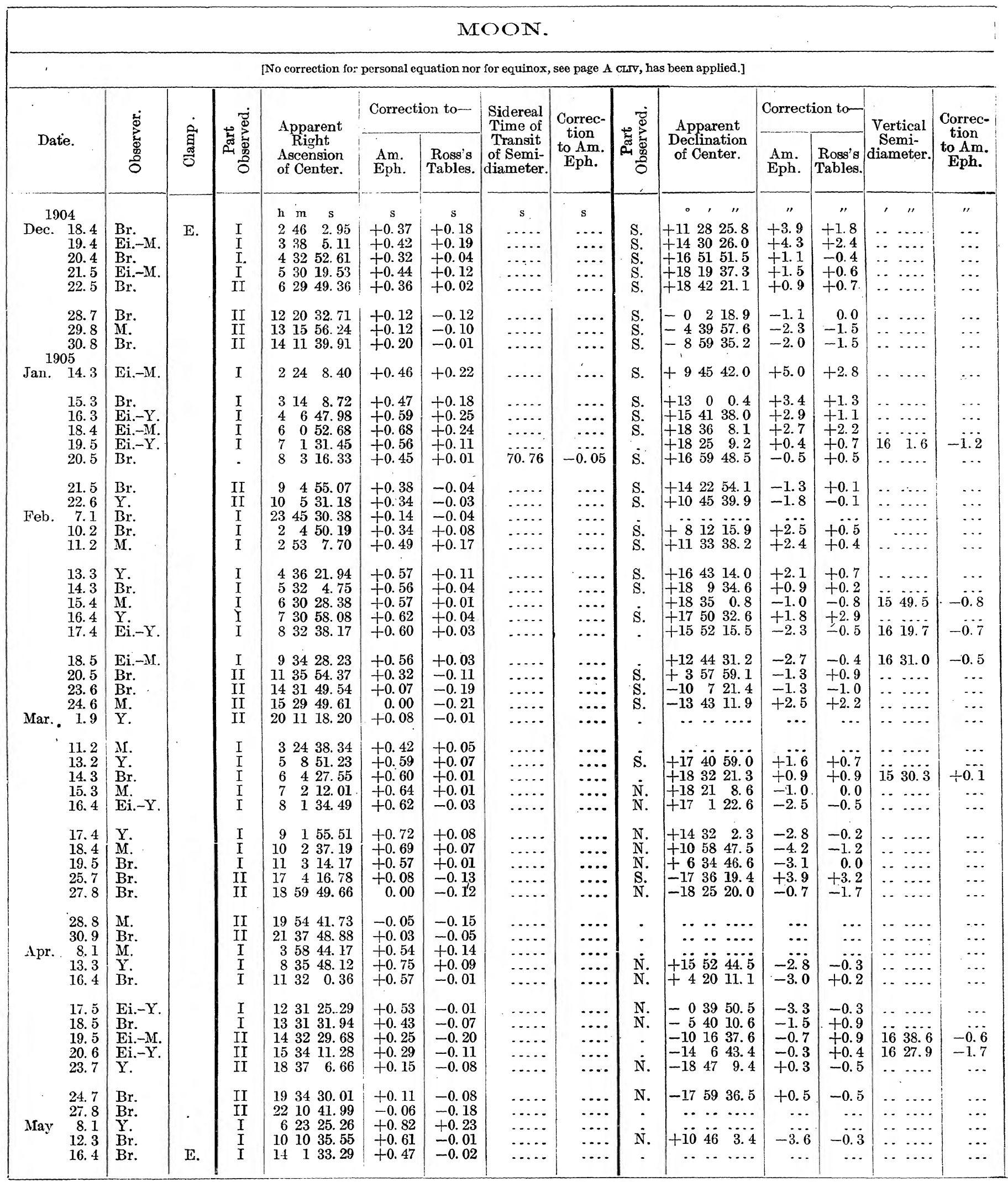




\begin{tabular}{|c|c|c|c|c|c|c|c|c|c|c|c|c|c|c|c|}
\hline \multicolumn{16}{|c|}{ MOON. } \\
\hline \multicolumn{16}{|c|}{ [No correction for personal equation nor for equinox, see page A cLrv, has heen applied.] } \\
\hline \multirow{2}{*}{\multicolumn{2}{|c|}{ Date. }} & \multirow{2}{*}{ 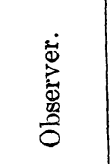 } & \multirow{2}{*}{ 突 } & \multirow{2}{*}{ 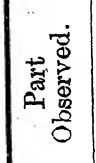 } & \multirow{2}{*}{\begin{tabular}{c|} 
Apparent \\
Right \\
Ascension of \\
of Center.
\end{tabular}} & \multicolumn{2}{|c|}{ Correction to- } & \multirow{2}{*}{$\begin{array}{c}\text { Sidereal } \\
\text { Time of } \\
\text { Transit } \\
\text { Semi- } \\
\text { diameter. }\end{array}$} & \multirow{2}{*}{$\begin{array}{c}\text { Correc- } \\
\text { tion } \\
\text { to Am. } \\
\text { Eph. }\end{array}$} & \multirow{2}{*}{ 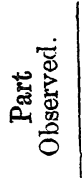 } & \multirow{2}{*}{$\begin{array}{c}\text { Apparent } \\
\text { Declination } \\
\text { of Center. }\end{array}$} & \multicolumn{2}{|c|}{ Correction to- } & \multirow{2}{*}{$\mid \begin{array}{c}\text { Vertical } \\
\text { Semi- } \\
\text { diameter. }\end{array}$} & \multirow{2}{*}{$\begin{array}{l}\text { Correc- } \\
\text { tion } \\
\text { to Am. } \\
\text { Eph. }\end{array}$} \\
\hline & & & & & & $\begin{array}{l}\text { Am. } \\
\text { Eph. }\end{array}$ & $\begin{array}{c}\text { Ross's } \\
\text { Tables. }\end{array}$ & & & & & Am. & $\begin{array}{l}\text { Ross's } \\
\text { Tables. }\end{array}$ & & \\
\hline \multirow{5}{*}{\multicolumn{2}{|c|}{\begin{tabular}{cc}
\multicolumn{2}{c|}{1905} \\
May 18.5 \\
19.6 \\
20.6 \\
21.6 \\
23.7
\end{tabular}}} & & & & $\mathrm{~h} \cdot \mathrm{m} \quad \mathrm{s}$ & s. & $\mathrm{s}$ & $\mathrm{s}$ & s & & $\circ, \quad$, & " & $\therefore$ & , , ' & " \\
\hline & & Br. & E. & II & 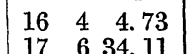 & +0.33 & $\begin{array}{l}-0.08 \\
-0.14\end{array}$ & $\cdots$ & $\cdots$ & $\mathrm{N}$. & $-\begin{array}{rrr}-17 & 07 & \ldots .8\end{array}$ & $+0 . i$ & $+0 . \dot{2}$ & $\cdots \cdots$ & $\cdots$ \\
\hline & & $\begin{array}{l}\text { El. }-\mathrm{X} \text {. } \\
\mathrm{MI} .\end{array}$ & & II & $\begin{array}{lll}17 & 6 & 34.11 \\
18 & 8 & 30.82\end{array}$ & $\begin{array}{l}+0.22 \\
+0.21\end{array}$ & $\begin{array}{l}-0.14 \\
-0.10\end{array}$ & $\cdots$ & $\cdots$ & N. & $\begin{array}{lll}-17 & 07 & 0.8 \\
-18 & 57 & 10.2\end{array}$ & $\begin{array}{l}+0.1 \\
-0.2\end{array}$ & $\begin{array}{l}+0.2 \\
-0.6\end{array}$ & $\begin{array}{ll}\cdots \\
\cdots\end{array}$ & $\begin{array}{l}\cdots \\
\cdots\end{array}$ \\
\hline & & $\mathrm{Br}$. & & II & $\begin{array}{llll}19 & 8 & 42.24\end{array}$ & +0.24 & -0.02 & $\cdots$ & $\cdots$ & $\mathrm{N}$. & $\begin{array}{llll}-18 & 41 & 55.6\end{array}$ & +0.9 & +0.2 & $\therefore \ldots$ & $\cdots$ \\
\hline & & M. & & II & $\begin{array}{lll}21 & 0 & 41.86\end{array}$ & 0.00 & -0.19 & & $\cdots$ & N. & $-15 \cdot 3 \quad 35.7$ & +2.3 & +1.4 & .. . . & $\cdots$ \\
\hline & 24.7 & $\mathrm{HI}$. & & II & & -0.04 & -0.20 & $\ldots$ & $\ldots$ & & $\because \ddot{0} \because \cdots$ & & & $\ldots \ldots$ & $\cdots$ \\
\hline June & $\begin{array}{r}9.2 \\
13.4\end{array}$ & $\begin{array}{l}\mathrm{Br} \\
\mathrm{Br}\end{array}$ & & I & $\begin{array}{lll}10 & 49 & 33.98 \\
14 & 35 & 46.48\end{array}$ & $\begin{array}{l}+0.54 \\
+0.44 \\
+0\end{array}$ & $\begin{array}{l}-0.08 \\
-0.05\end{array}$ & $\cdots$ & $\cdots$ & $\stackrel{N}{N}$. & 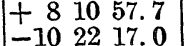 & $\begin{array}{l}-4.1 \\
-2.2\end{array}$ & $\begin{array}{l}-0.8 \\
-0.3\end{array}$ & $\cdots \cdots$ & $\cdots$ \\
\hline & $\begin{array}{l}13.4 \\
14.4\end{array}$ & Ei.-Y. & & I & $\begin{array}{lll}14 & 50 & 40.40 \\
15 & 35 & 31.71\end{array}$ & $\begin{array}{l}+0.44 \\
+0.47\end{array}$ & $\begin{array}{l}-0.03 \\
+0.02\end{array}$ & $\cdots$ & $\cdots$ & N. & $\begin{array}{llll}-10 & 22 & 1.0 \\
-14 & 10 & 23.0\end{array}$ & $\begin{array}{l}-2.2 \\
-1.5\end{array}$ & $\begin{array}{l}-0.3 \\
-0.3\end{array}$ & \begin{tabular}{ll|}
$\cdots$ & $\cdots$ \\
$\cdots$ & $\cdots$
\end{tabular} & $\begin{array}{l}\cdots \\
\cdots\end{array}$ \\
\hline & 15.5 & M. & & I & $16 \quad 36 \quad 44.26$ & +0.32 & -0.09 & $\cdots$ & $\cdots$ & N. & $\begin{array}{lll}-17 & 1 & 9.6\end{array} \mid$ & -2.6 & -2.0 & $\cdots \cdots$ & $\cdots$ \\
\hline & 16.5 & $\mathrm{Br}$. & & & $1738 \quad 37.49$ & +0.40 & +0.04 & 70.91 & +0.04 & N. & $\begin{array}{lll}-18 & 41 & 17.4\end{array}$ & -1.2 & -1.2 & $\ldots \ldots$ & $\ldots$ \\
\hline & 18.6 & Ei.-Y. & & II & $\begin{array}{llll}19 & 39 & 33.58\end{array}$ & $+0,18$ & -0.09 & $\ldots$ & $\ldots$ & N. & $\begin{array}{llll}-18 & 15 & 50.1\end{array}$ & +0.5 & -0.1 & $\therefore \ldots$. & $\cdots$ \\
\hline Aug & $\begin{array}{l}19.6 \\
15.5\end{array}$ & Hl. & $\frac{\mathrm{E}}{\mathrm{W}}$ & II & $\begin{array}{llll}20 & 36 & 27.59 \\
2 & 9 & 27 & 9\end{array}$ & +0.04 & -0.19 & $\cdots$ & $\cdots$ & N. & $\left|\begin{array}{rrr}-16 & 23 & 16.3\end{array}\right|$ & +1.0 & +0.3 & $\cdots \cdots$ & $\cdots$ \\
\hline & $\begin{array}{l}15.5 \\
17.6\end{array}$ & Br. & & iI & \begin{tabular}{rrr|}
22 & 29 & 37.28 \\
0 & 6 & 5.37
\end{tabular} & $\begin{array}{l}+0.03 \\
+0.15\end{array}$ & $\begin{array}{l}-0.21 \\
-0.04\end{array}$ & $\cdots$ & $\cdots$ & $\begin{array}{l}\text { N. } \\
\text { N. }\end{array}$ & $\begin{array}{lll}-9 & 58 & 45.8 \\
-2 & 15 & 7.8\end{array}$ & $\begin{array}{l}-1.4 \\
+2.1\end{array} \mid$ & $\begin{array}{l}-2.5 \\
+1.0\end{array}$ & $\begin{array}{lll}\cdots & \cdots \\
\cdots & \cdots\end{array}$ & $\cdots$ \\
\hline & 18.6 & Hl. & & II & $\begin{array}{llll}0 & 52 & 39.97\end{array}$ & +0.03 & -0.16 & $\ldots$ & $\ldots$ & N. & +14518.7 & +0.5 & -0.7 & $\ldots \ldots$ & ... \\
\hline & 21.7 & $\mathrm{Br}$. & & II & $\begin{array}{llll}3 & 13 & 56.44\end{array}$ & $\begin{array}{r}0.09 \\
+0.09 \\
\end{array}$ & -0.17 & $\cdots$ & $\cdots$ & N. & +123617.8 & +1.8 & +0.4 & $\therefore \cdots$ & $\cdots$ \\
\hline & 22.7 & Hl. & & II & $\begin{array}{lll}4 & 3 & 43.57\end{array}$ & +0.10 & -0.21 & $\cdots$ & $\cdots$ & $N$ & 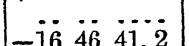 & $\left|\begin{array}{lll}-1 & 4\end{array}\right|$ & $-\ddot{i}$ & $\cdots \cdots$ & $\cdots$ \\
\hline Sept. & $\begin{array}{l}5.2 \\
6.3\end{array}$ & $\begin{array}{l}\text { Hl. } \\
\text { Hil. }\end{array}$ & & $\dot{\mathrm{I}}$ & $\ddot{i 7} \ddot{35} \quad \ddot{4.90}$ & $+\dddot{0.0 i}$ & $-\ddot{0} . \overline{2}$ & $\cdots$ & $\cdots$ & N. & $\mid \begin{array}{lll}-16 & 46 & 41.2 \\
-18 & 29 & 37.0\end{array}$ & $\begin{array}{l}-1.4 \\
+0.3\end{array}$ & $\begin{array}{l}-1.2 \\
-0.1\end{array}$ & $\begin{array}{ll}\cdots & \cdots \\
\cdots & \cdots\end{array}$ & $\cdots$ \\
\hline & 7.3 & Bs. & & $I$ & $\begin{array}{lll}18 & 33 & 52.02\end{array}$ & +0.33 & -0.09 & & & & & & & $\ldots \ldots$ & $\cdots$ \\
\hline & 8.3 & $\mathrm{Hl}$. & & I & $\begin{array}{llll}19 & 31 & 21.73\end{array}$ & +0.28 & -0.11 & $\cdots$ & $\cdots$ & $\dot{\mathrm{S}}$. & $\mid \begin{array}{lll}-18 & 27 & 032\end{array}$ & +42 & +3.0 & .. $\ldots$ & $\ldots$ \\
\hline & $\begin{array}{r}9.4 \\
12.5\end{array}$ & $\begin{array}{l}\text { Bs. } \\
\text { Bs. }\end{array}$ & & $\begin{array}{l}\mathrm{I} \\
\mathrm{I}\end{array}$ & $\begin{array}{rrr}20 & 26 & 59.26 \\
23 & 0 & 55.89\end{array}$ & $\begin{array}{l}+0.38 \\
+0.28\end{array}$ & $\begin{array}{l}+0.02 \\
+0.02\end{array}$ & $\cdots$ & $\cdots$ & S. & $\left|\begin{array}{rrrr}-16 & 50 & 34.8 \\
. & . & \ldots . .\end{array}\right|$ & +1.2 & -0.2 & $\cdots \cdots$ & $\cdots$ \\
\hline & 13.5 & Bs. & & . & $\cdots \cdots$ & $\ldots$ & $\ldots$ & $\cdots$ & $\because$ & : & $-\begin{array}{llll}-3 & \ddot{4} 2 & 39.7\end{array}$ & $+\ddot{2} .9$ & $+\ddot{1.4}$ & i4 $\ddot{50.5}$ & $-\ddot{1 . j 5}$ \\
\hline & 15.6 & Bs. & & II & $\begin{array}{lll}121 & 51.98\end{array}$ & +0.27 & +0.05 & $\ldots$ & $\ldots$ & N. & +41619.9 & +0.2 & -1.4 & $\ldots \ldots$ & $\cdots$ \\
\hline & 18.7 & Bs. & & II & $\begin{array}{l}34433.59 \\
\end{array}$ & $\begin{array}{l}+0.28 \\
+\end{array}$ & $\begin{array}{l}-0.02 \\
-0.14\end{array}$ & $\cdots$ & $\cdots$ & N. & +142713.1 & $+0 . \overline{7}$ & -0.7 & . . . & $\cdots$ \\
\hline & $\begin{array}{l}21.8 \\
22.8\end{array}$ & $\begin{array}{l}\text { Hl. } \\
\text { Bs. }\end{array}$ & & II & $\begin{array}{ccc}6 & 21 & 47.01 \\
\ldots & \ldots & \ldots . .\end{array}$ & $\begin{array}{r}+0.30 \\
\ldots . .\end{array}$ & $\begin{array}{r}-0.14 \\
\ldots\end{array}$ & $\cdots$ & $\cdots$ & $\dot{\mathrm{s}}$ & 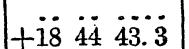 & $+\ddot{2.0}$ & +3.0 & $\begin{array}{ll}\cdots & \cdots \\
\cdots & \cdots\end{array}$ & $\cdots$ \\
\hline & 23.8 & $\mathrm{Hl}$. & & 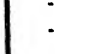 & $\cdots \cdots$ & $\cdots$ & $\cdots$ & & $\cdots$ & S. & $+17 \quad 18 \quad 19.9$ & +0.3 & +2.0 & $\cdots \cdots$ & $\cdots$ \\
\hline & 24.9 & Bs. & & II & $\begin{array}{llll}9 & 14 & 2.75\end{array}$ & +0.42 & -0.05 & $\ldots$ & $\ldots$ & - & $\ldots \ldots \ldots$ & $\ldots$ & $\cdots$ & $\ldots \ldots$ & $\cdots$ \\
\hline Oct. & $\begin{array}{r}25.9 \\
4.2\end{array}$ & $\begin{array}{l}\text { Hl. } \\
\text { Bs. }\end{array}$ & & II & $\begin{array}{llll}10 & 12 & 56.10 \\
18 & 14 & 53 & 86\end{array}$ & $\begin{array}{l}+0.36 \\
+0.31\end{array}$ & $\begin{array}{l}-0.09 \\
-0.05\end{array}$ & $\cdots$ & $\cdots$ & $\dot{s}$ & 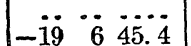 & $+\ddot{3} 5$ & $+\ddot{2} \ddot{7}$ & $\cdots$ & $\cdots$ \\
\hline & $\begin{array}{l}4.2 \\
5.3\end{array}$ & Hi. & & 1 & $\begin{array}{lll}18 & 14 & 03.80 \\
19 & 13 & 43.10\end{array}$ & $\begin{array}{l}+0.31 \\
+0.28\end{array}$ & $\begin{array}{l}-0.00 \\
-0.06\end{array}$ & $\cdots$ & $\cdots$ & S. & $\left|\begin{array}{llll}-19 & 0 & 40.4 \\
-18 & 52 & 13.4\end{array}\right|$ & $\begin{array}{l}+0.0 \\
+4.8\end{array}$ & +3.7 & $\begin{array}{lll}\cdots & \cdots \\
\cdots & \cdots & \cdots \\
\end{array}$ & $\cdots$ \\
\hline & 6.3 & $\mathrm{Br}$. & & $I$ & $\begin{array}{llll}20 & 10 & 16.81\end{array}$ & +0.25 & -0.07 & & & s. & $-17 \begin{array}{lll}-17 & 36.0\end{array} \mid$ & +2.1 & +0.7 & $\because \cdots$ & $\cdots$ \\
\hline & 7.3 & Bs. & & I & $\begin{array}{lll}21 & 4 & 18.27\end{array}$ & +0.27 & -0.03 & $\ldots$ & $\ldots$ & S. & $-15 \quad 18 \quad 16.5$ & +2.8 & +1.3 & $\ldots \ldots$ & $\cdots$ \\
\hline & 8.4 & Bs. & & I & 215551.48 & +0.26 & -0.02 & & $\cdots$ & S. & -122057.6 & +2.9 & +1.3 & $\ldots \ldots$ & $\cdots$ \\
\hline & $\begin{array}{r}9.4 \\
725\end{array}$ & Hl. & & I & $\begin{array}{rrr}22 & 45 & 16.68 \\
1 & 6 & 5.58\end{array}$ & $\begin{array}{l}+0.28 \\
+0.28\end{array}$ & $\begin{array}{l}+0.02 \\
+0.05\end{array}$ & 60.90 & -0.07 & S. & 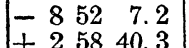 & $\begin{array}{l}+4.4 \\
+4.3\end{array}$ & +2.8 & $\cdots \cdots$ & $\cdots$ \\
\hline & 13.5 & Bs. & & II & $\begin{array}{lrr}1 & 0 & 0.08 \\
1 & 52 & 31.16\end{array}$ & $\begin{array}{l}+0.28 \\
+0.24\end{array}$ & $\begin{array}{r}+0.00 \\
0.00\end{array}$ & & -0.06 & : & 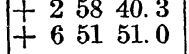 & $\begin{array}{l}+4.0 \\
+3.5\end{array}$ & $\begin{array}{l}+2.5 \\
+1.6\end{array}$ & $\ddot{14} \ddot{4} \ddot{1} .9$ & -1.7 \\
\hline & 14.5 & Bs. & & II & $2 \quad 3937.14$ & +0.22 & -0.04 & & ... & - & $+1028 \quad 3.1$ & +2.6 & +0.8 & 1443.2 & -0.7 \\
\hline & 15.6 & $\mathrm{Hl}$. & & II & $\begin{array}{lll}3 & 27 & 50.78\end{array}$ & +0.09 & -0.21 & $\therefore$ & ... & . & +133823.2 & +1.5 & -0.2 & 1443.8 & -2.1 \\
\hline & 16.6 & $\mathrm{Br}$. & & II & $\begin{array}{lll}4 & 17 & 32.88\end{array}$ & +0.27 & -0.07 & $\cdots$ & $\cdots$ & $\dot{s}$ & $\begin{array}{lll}+16 & 14 & 3.4\end{array}$ & +1.3 & -0.1 & 1450.6 & +0.7 \\
\hline & $\begin{array}{l}21.8 \\
22.8\end{array}$ & $\begin{array}{l}\text { Hl. } \\
\text { Hl. }\end{array}$ & & $\begin{array}{l}11 \\
\text { II }\end{array}$ & $\begin{array}{llr}8 & 48 & 15.71 \\
9 & 45 & 5.43\end{array}$ & $\begin{array}{l}+0.34 \\
+0.32\end{array}$ & $\begin{array}{l}-0.14 \\
-0.14\end{array}$ & $\cdots$ & $\cdots$ & S. & $\mid \begin{array}{rrrr}+16 & 11 & 35.9 \\
+13 & 8 & 44.2\end{array}$ & $\begin{array}{l}-0.2 \\
-1.8\end{array}$ & $\begin{array}{l}+2.0 \\
+0.9\end{array}$ & $\begin{array}{ll}\cdots & \cdots \\
\cdots & \cdots \\
\end{array}$ & $\cdots$ \\
\hline Nov. & & Bs. & & I & $1851 \quad 2.15$ & +0.32 & +0.03 & $\ldots$ & $\ldots$. & $\mathrm{S}$. & $-19 \begin{array}{lll}-19 & 25.3\end{array}$ & +6.2 & +5.4 & & \\
\hline & $\begin{array}{l}1.2 \\
2.2\end{array}$ & Hil. & & $\mathrm{I}$ & $\begin{array}{llll}10 & 50 & 18.12\end{array}$ & $\begin{array}{l}+0.32 \\
+0.26 \\
\end{array}$ & -0.01 & $\cdots \cdots$ & & S. & $\left.\begin{array}{lll}-19 & 18 & 20.5 \\
-18 & 21 & 53.5\end{array}\right]$ & $\begin{array}{l}+0.2 \\
+1.2\end{array}$ & $\begin{array}{l}+3.4 \\
+0.2\end{array}$ & $\begin{array}{lll}\cdots & \cdots \\
\cdots & \cdots\end{array}$ & $\cdots$ \\
\hline & 6.3 & Bs. & & $\mathrm{I}$ & $\begin{array}{lll}23 & 18 & 9.00\end{array}$ & +0.30 & +0.09 & $\cdots$ & .. & S. & -62636.1 & +3.3 & +1.8 & $\ldots \ldots$ & $\cdots$ \\
\hline & 7.4 & Hil. & & I & $\begin{array}{lll}0 & 4 & 57.97\end{array}$ & +0.31 & +0.11 & $\cdots$ & .. & S. & -22725.6 & $\begin{array}{r}+6.0 \\
\end{array}$ & +4.4 & $\cdots \cdots$ & $\cdots$ \\
\hline & 8.4 & Bs. & & I & $\begin{array}{lll}0 & 51 & 5.92\end{array}$ & +0.28 & +0.08 & & & S. & +13551.2 & +3.9 & +2.1 & $\cdots \cdots$ & $\cdots$ \\
\hline & 10.5 & Bs. & & I & $\begin{array}{lll}2 & 24 & 0.47\end{array}$ & +0.36 & +0.12 & & & S. & +92013.3 & +3.8 & +1.8 & .. . . . & $\cdots$ \\
\hline & $\begin{array}{l}11.5 \\
12.5\end{array}$ & Hl. & & $\dot{\text { II }}$ & \begin{tabular}{rrr|}
3 & 11 & 54.70 \\
4 & 1 & 19.26
\end{tabular} & & $\begin{array}{l}-0.09 \\
-0.17\end{array}$ & 62.50 & -0.08 & $\cdot$ & $+\ddot{15} \ddot{34} \quad \ddot{5} \ddot{i}$ & $+\ddot{0} .8$ & $-\ddot{1} .0$ & i4 $\ddot{4} \overrightarrow{7.6}$ & $-\ddot{1.3}$ \\
\hline & 14.6 & Bs. & & II & \begin{tabular}{rrr|}
4 & 1 & 19.26 \\
5 & 45 & 4.49
\end{tabular} & $\begin{array}{l}+0.13 \\
+0.44\end{array}$ & $\begin{array}{l}-0.17 \\
+0.03\end{array}$ & $\cdots$ & $\cdots$ & $\dot{\mathrm{S}}$. & $\begin{array}{rrr}+10 & 44 & 0.1 \\
+19 & 6 & 13.5\end{array}$ & $\begin{array}{l}+0.0 \\
+0.2\end{array}$ & $\begin{array}{l}-1.0 \\
-0.5\end{array}$ & $\begin{array}{lll}14 & \pm 1 & 0 \\
\ldots & \ldots\end{array}$ & $\ldots$ \\
\hline & 16.7 & $\mathrm{Br}$. & W. & II & $\begin{array}{lll}7 & 33 & 51.96\end{array}$ & +0.50 & +0.03 & $\cdots \cdot$ & $\cdots$ & $\tilde{S}$. & +185413.7 & +1.1 & +2.1 & $\ldots$. & \\
\hline
\end{tabular}




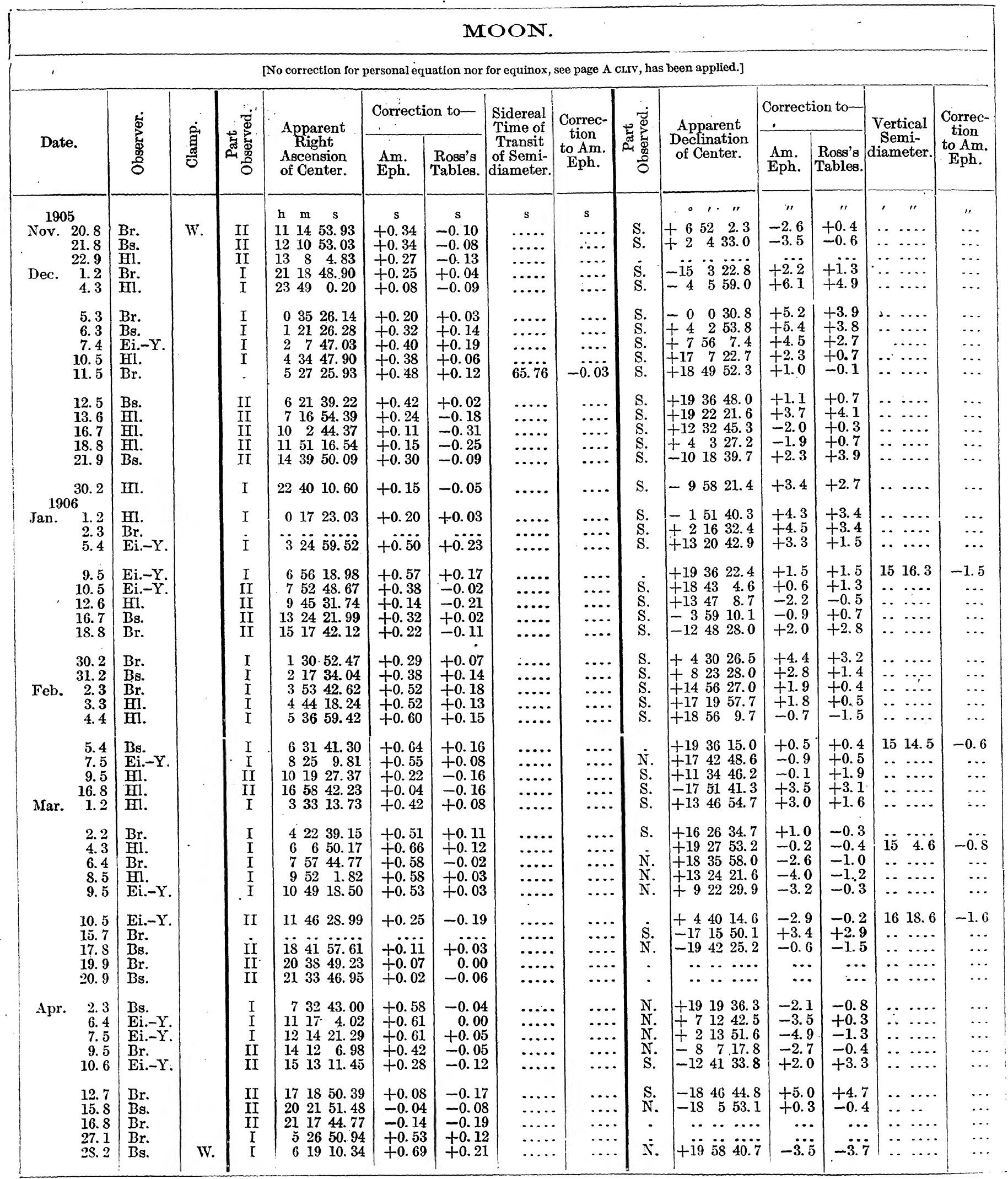


MOON.

[No correction for personal equation nor for equinox, see page A cLrv, has been applied.]

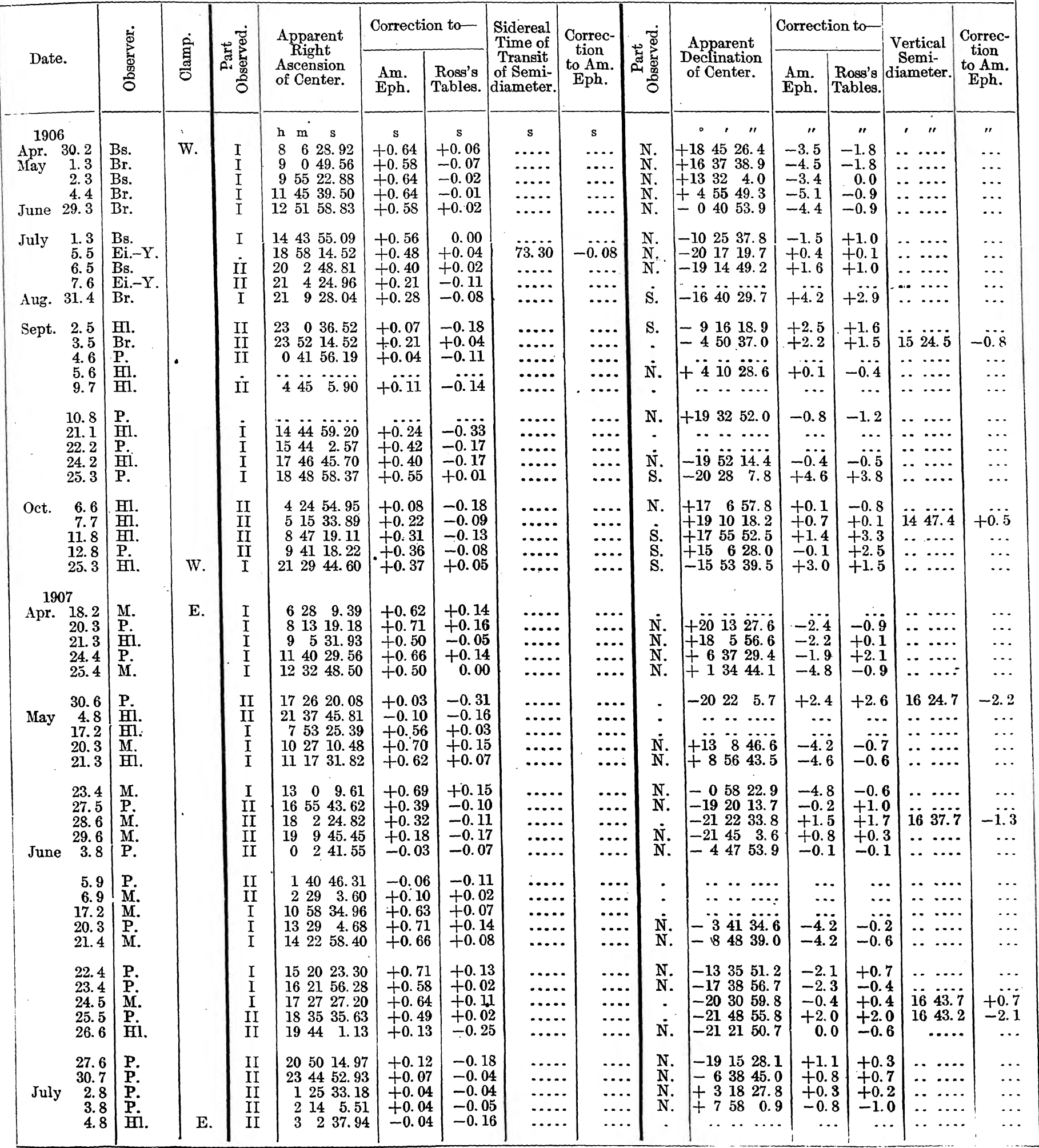

$29297^{\circ}-20-32$ 


\begin{tabular}{|c|c|c|c|c|c|c|c|c|c|c|c|c|c|c|c|}
\hline \multicolumn{16}{|c|}{ MOON. } \\
\hline & & & & & [No correction for $\mathrm{p}$ & personal eq & fuation nor fo & for equinox, s & see page A C & curv, has & s been applied.] & & & & \\
\hline \multirow{2}{*}{\multicolumn{2}{|c|}{ Date. }} & \multirow{2}{*}{ 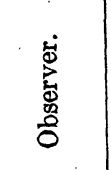 } & \multirow{2}{*}{ 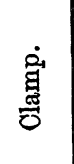 } & \multirow{2}{*}{ 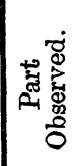 } & \multirow{2}{*}{$\begin{array}{l}\text { Apparent } \\
\text { Right } \\
\text { Ascension } \\
\text { of Center. }\end{array}$} & \multicolumn{2}{|c|}{ Correction to- } & \multirow{2}{*}{$\begin{array}{c}\text { Sidereal } \\
\text { Time of } \\
\text { Transit } \\
\text { of Semi- } \\
\text { diameter. }\end{array}$} & \multirow{2}{*}{$\begin{array}{c}\text { Correc- } \\
\text { tion } \\
\text { to Am. } \\
\text { Eph. }\end{array}$} & \multirow{2}{*}{ 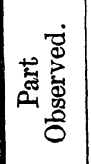 } & \multirow{2}{*}{$\begin{array}{c}\text { Apparent } \\
\text { Declination } \\
\text { of Center. }\end{array}$} & \multicolumn{2}{|c|}{ Correction to- } & \multirow{2}{*}{$\begin{array}{c}\text { Vertical } \\
\text { Semi- } \\
\text { diameter. }\end{array}$} & \multirow{2}{*}{$\begin{array}{l}\text { Correc- } \\
\text { tion } \\
\text { to Am. } \\
\text { Eph. }\end{array}$} \\
\hline & & & & & & $\begin{array}{l}\text { Am. } \\
\text { Eph. }\end{array}$ & $\begin{array}{c}\text { Ross's } \\
\text { Tables. }\end{array}$ & & & & & $\begin{array}{l}\text { Am. } \\
\text { Eph. }\end{array}$ & $\begin{array}{c}\text { Ross's } \\
\text { Tables }\end{array}$ & & \\
\hline \multicolumn{2}{|c|}{\begin{tabular}{cr}
\multicolumn{2}{c|}{1907} \\
July & 5.9 \\
15.2 \\
16.2 \\
19.3 \\
20.3
\end{tabular}} & $\begin{array}{l}\text { M. } \\
\text { Hi. } \\
\text { M. } \\
\text { M. } \\
\text { M. }\end{array}$ & E. & $\begin{array}{l}\text { II } \\
\text { I } \\
\text { I } \\
\text { I } \\
\text { I }\end{array}$ & $\begin{array}{rrc}\mathrm{h} & \mathrm{m} & \mathrm{s} \\
3 & 51 & 50.24 \\
11 & 31 & 32.00 \\
12 & 20 & 15.34 \\
14 & 54 & 53.77 \\
15 & 52 & 34.54\end{array}$ & $\begin{array}{l}\text { 's } \\
+0.02 \\
+0.62 \\
+0.68 \\
+0.61 \\
+0.66\end{array}$ & $\begin{array}{c}\mathrm{s} \\
-0.16 \\
+0.06 \\
+0.11 \\
-0.01 \\
+0.04\end{array}$ & $\begin{array}{c}\text { s } \\
\cdots \cdots \\
\cdots \cdots \cdot \\
\cdots \cdots \\
\cdots \cdots \\
\cdots \cdots\end{array}$ & $\begin{array}{l}\mathrm{s} \\
\ldots \ldots \\
\ldots \ldots \\
\ldots \ldots \\
\ldots \ldots \\
\ldots\end{array}$ & $\begin{array}{l}\dot{-} \\
\dot{\mathrm{N}} . \\
\mathrm{N} .\end{array}$ & 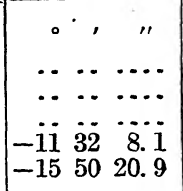 & $\begin{array}{c}\prime \prime \\
\ldots \\
\cdots \\
-3.3 \\
-2.7\end{array}$ & $\begin{array}{c}\prime \prime \\
\ldots . \\
\cdots \\
\ddot{0.1} \\
-0.4\end{array}$ & 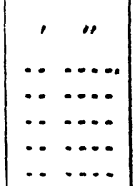 & \begin{tabular}{l|}
$\prime \prime$ \\
$\ldots$ \\
$\cdots$ \\
$\cdots$ \\
$\cdots$ \\
$\cdots$
\end{tabular} \\
\hline & $\begin{array}{l}21.4 \\
23.5 \\
25.5 \\
26.6 \\
27.6\end{array}$ & $\begin{array}{l}\text { Hl. } \\
\text { P. } \\
\text { Hil. } \\
\text { P. } \\
\text { Hil. }\end{array}$ & & $\begin{array}{l}\text { I* } \\
\text { I } \\
\text { II } \\
\text { II } \\
\text { II }\end{array}$ & $\begin{array}{rrr}16 & 54 & 27.24 \\
19 & 8 & 7.95 \\
21 & 21 & 31.44 \\
22 & 23 & 13.05 \\
23 & 20 & 50 . .86\end{array}$ & $\begin{array}{l}+0.87 \\
+0.54 \\
+0.10 \\
+0.05 \\
+0.15\end{array}$ & $\begin{array}{l}+0.27 \\
+0.06 \\
-0.21 \\
-0.18 \\
-0.02\end{array}$ & $\begin{array}{l}\cdots \cdots \cdot \\
\cdots \cdots \cdot \\
\cdots \cdots \cdot \\
\cdots \cdots \\
\cdots \cdots\end{array}$ & $\begin{array}{l}\ldots . . \\
\cdots \cdots \\
\cdots \cdots \\
\cdots \cdots\end{array}$ & $\begin{array}{l}\text { N. } \\
\dot{\text { N. }} \\
\text { N. }\end{array}$ & $\begin{array}{rrr}-19 & 14 & 43.0 \\
-21 & 49 & 4.2 \\
-17 & 42 & 53.4 \\
-13 & 40 & 30.8 \\
-8 & 52 & 53.6\end{array}$ & $\begin{array}{l}-2.0 \\
+1.4 \\
+1.8 \\
+2.1 \\
-1.3\end{array}$ & $\begin{array}{r}-0.7 \\
+0.9 \\
+0.8 \\
+1.4 \\
-1.7\end{array}$ & $\begin{array}{cc}16 & 41.3 \\
16 & 33.1 \\
\ldots & \cdots \\
\ldots & \cdots\end{array}$ & $\begin{array}{r}-\ddot{1} . \overline{1} \\
-1.9 \\
\cdots \\
\cdots\end{array}$ \\
\hline Aug. & $\begin{array}{r}29.7 \\
30.7 \\
1.8 \\
2.8 \\
14.2\end{array}$ & $\begin{array}{l}\mathrm{Hl} . \\
\mathrm{P} . \\
\mathrm{Hi} . \\
\mathrm{P} . \\
\mathrm{Hl} .\end{array}$ & & $\begin{array}{l}\text { II } \\
\text { II } \\
\text { II } \\
\text { II } \\
\text { I }\end{array}$ & $\begin{array}{rrr}1 & 6 & 28.01 \\
1 & 56 & 24.44 \\
3 & -35 & 8.15 \\
4 & 25 & 15.74 \\
13 & 44 & 9.53\end{array}$ & $\begin{array}{l}-0.05 \\
+0.08 \\
-0.13 \\
+0.17 \\
+0.56\end{array}$ & $\begin{array}{l}-0.16 \\
-0.03 \\
-0.29 \\
-0.04 \\
-0.04\end{array}$ & $\begin{array}{l}\cdots \ldots . \\
\cdots \ldots . \\
\cdots \ldots . \\
\cdots \cdots \\
\cdots\end{array}$ & $\begin{array}{l}\ldots . . \\
\ldots . . \\
\ldots . . \\
\ldots . .\end{array}$ & $\begin{array}{l}\text { N. } \\
\text { N. } \\
\text { N. } \\
\text { : }\end{array}$ & 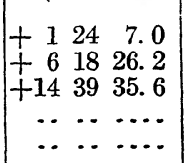 & $\begin{array}{r}-1.3 \\
+0.4 \\
-1.4 \\
\ldots . \\
\ldots\end{array}$ & $\begin{array}{c}-1.3 \\
+0.5 \\
-1.8 \\
\cdots \\
\cdots\end{array}$ & \begin{tabular}{ll|} 
& \\
$\ldots$ & $\ldots$ \\
$\cdots$ & $\ldots$ \\
$\ldots$ & $\ldots$ \\
$\ldots$ & $\ldots$ \\
$\ldots$ & $\ldots$
\end{tabular} & $\begin{array}{l}\cdots \\
\cdots \\
\cdots \\
\cdots\end{array}$ \\
\hline & $\begin{array}{l}15.2 \\
18.3 \\
19.4 \\
20.4 \\
22.5\end{array}$ & $\begin{array}{l}\mathrm{P} . \\
\mathrm{P} . \\
\mathrm{P} . \\
\mathrm{P} . \\
\mathrm{P} .\end{array}$ & & $\begin{array}{l}\text { I } \\
\text { I } \\
\text { I } \\
\text { I } \\
\text { I }\end{array}$ & $\begin{array}{rrr}14 & 36 & 4.16 \\
17 & 31 & 12.50 \\
18 & 36 & 16.36 \\
19 & 42 & 45.13 \\
21 & 51 & 54.86\end{array}$ & $\begin{array}{l}+0.62 \\
+0.88 \\
+0.64 \\
+0.65 \\
+0.35\end{array}$ & $\begin{array}{l}-0.01 \\
+0.23 \\
+0.04 \\
+0.13 \\
+0.01\end{array}$ & $\begin{array}{l}\ldots . . \\
\cdots \cdots \cdot \\
\cdots \cdots \cdot \\
\cdots \cdots\end{array}$ & \begin{tabular}{l|}
$\ldots .$. \\
$\cdots \cdots$ \\
$\cdots \cdots$ \\
$\cdots \cdots$
\end{tabular} & $\begin{array}{l}\text { N. } \\
\text { N. } \\
\text { S. } \\
\text { S. }\end{array}$ & $\begin{array}{rrr}-10 & 0 & 35.9 \\
-20 & 40 & 53.3 \\
-21 & 51 & 42.4 \\
-21 & 25 & 5.6 \\
-15 & 51 & 35.5\end{array}$ & $\begin{array}{l}-3.5 \\
-0.2 \\
+2.2 \\
+2.3 \\
+4.4\end{array}$ & $\begin{array}{l}-0.2 \\
+0.4 \\
+1.8 \\
+1.4 \\
+3.1\end{array}$ & $\begin{array}{lll}\ldots & \ldots \\
16 & 26.7 \\
\ldots & \ldots & \\
\ldots & \ldots & \end{array}$ & $\begin{array}{r}\ldots \\
-1.1 \\
\cdots \\
\cdots\end{array}$ \\
\hline & $\begin{array}{l}24.6 \\
25.6 \\
26.6 \\
28.7 \\
29.7\end{array}$ & $\begin{array}{l}\text { P. } \\
\text { Hi. } \\
\text { P. } \\
\text { M. } \\
\text { Hi. }\end{array}$ & & $\begin{array}{l}\text { II } \\
\text { II } \\
\text { II } \\
\text { II } \\
\text { II }\end{array}$ & $\begin{array}{rrrr}23 & 48 & 32.44 \\
0 & 42 & 21.39 \\
1 & 34 & 12.64 \\
3 & 15 & 21.98 \\
4 & 6 & 2.23\end{array}$ & $\begin{array}{l}+0.22 \\
-0.08 \\
+0.19 \\
+0.08 \\
-0.05\end{array}$ & $\begin{array}{l}+0.02 \\
-0.24 \\
+0.05 \\
-0.07 \\
-0.23\end{array}$ & $\begin{array}{l}\ldots \ldots . \\
\cdots \ldots . . \\
\cdots \cdots \\
\cdots \cdots\end{array}$ & $\begin{array}{l}\cdots . . \\
\cdots \cdots \\
\cdots \cdots \\
\cdots \cdots\end{array}$ & $\begin{array}{l}\text { N. } \\
\text { N. } \\
\text { N. } \\
\text { N: }\end{array}$ & 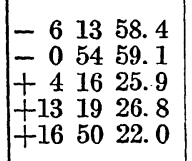 & $\begin{array}{l}+0.5 \\
+1.6 \\
+0.2 \\
-1.1 \\
-0.1\end{array}$ & $\begin{array}{l}-0.1 \\
+1.3 \\
+0.1 \\
-1.3 \\
-0.4\end{array}$ & \begin{tabular}{cc}
$\ldots$ & $\cdots$ \\
$\cdots$ & $\cdots$ \\
$\cdots$ & $\cdots$ \\
\hdashline. & $\cdots$ \\
$\cdots$ & $\cdots$ \\
\end{tabular} & $\begin{array}{l}\cdots \\
\cdots \\
\cdots \\
\cdots\end{array}$ \\
\hline Sept. & \begin{tabular}{l|l|} 
& \\
30.8 & \\
31.8 & \\
12.2 & \\
33.2 & 14.2
\end{tabular} & $\begin{array}{l}\mathrm{M} . \\
\mathrm{M} . \\
\mathrm{M} . \\
\mathrm{Hi} . \\
\mathrm{P} .\end{array}$ & & $\begin{array}{l}\text { II } \\
\text { II } \\
\text { I } \\
\text { I } \\
\text { I }\end{array}$ & $\begin{array}{rrr}4 & 57 & 20.15 \\
5 & 49 & 20.96 \\
15 & 13 & 48.48 \\
16 & 10 & 25.89 \\
17 & 10 & 11.27\end{array}$ & $\begin{array}{l}+0.15 \\
+0.22 \\
+0.59 \\
+0.56 \\
+0.93\end{array}$ & $\begin{array}{r}-0.09 \\
-0.07 \\
0.00 \\
-0.08 \\
+0.28\end{array}$ & $\ldots$. & $\begin{array}{l}\ldots \\
\ldots \ldots \\
\ldots \ldots \\
\ldots \ldots \\
\ldots \ldots\end{array}$ & $\begin{array}{l}\text { N. } \\
\dot{-} \\
\dot{N} . \\
\text { N. }\end{array}$ & $\left|\begin{array}{rrr}+19 & 30 & 31.2 \\
\ldots & \ldots & \ldots . . \\
-17 & 0 & \ldots . .7 \\
-20 & 8 & 53.7\end{array}\right|$ & $\begin{array}{r}-0.8 \\
\ldots . \\
-2.5 \\
-1.3\end{array}$ & $\begin{array}{r}-1.2 \\
\ldots . \\
-0.7 \\
-0.5\end{array}$ & \begin{tabular}{ll|} 
& \\
$\cdots$ & $\cdots$ \\
$\ldots$ & $\cdots$ \\
$\cdots$ & $\cdots$ \\
$\cdots$ & $\cdots$ \\
$\ldots$ & $\cdots$
\end{tabular} & $\begin{array}{l}\cdots \\
\cdots \\
\cdots \\
\cdots\end{array}$ \\
\hline & $\begin{array}{l}15.3 \\
16.3 \\
20.5 \\
21.5 \\
23.6\end{array}$ & $\begin{array}{l}\text { Hl.-P. } \\
\text { M. } \\
\text { P. } \\
\text { P. } \\
\text { Hi. }\end{array}$ & 1. & $\begin{array}{l}\text { I } \\
-I \\
\text { I } \\
\text { II } \\
\text { II }\end{array}$ & $\begin{array}{rrr}18 & 12 & 41.55 \\
19 & 16 & 51.09 \\
23 & 21 & 20.18 \\
0 & 16 & 11.52 \\
2 & 0 & 59.72\end{array}$ & $\begin{array}{l}+0.84 \\
+0.65 \\
+0.34 \\
+0.22 \\
+0.06\end{array}$ & $\begin{array}{l}+0.20 \\
+0.05 \\
+0.03 \\
-0.04 \\
-0.13\end{array}$ & \begin{tabular}{l|}
$\ldots \ldots$. \\
$\cdots \cdots \cdot$ \\
$\cdots \cdots$ \\
$\cdots \cdots$
\end{tabular} & $\begin{array}{l}\ldots . . \\
\cdots . . \\
\cdots . . \\
\cdots\end{array}$ & $\begin{array}{l}\dot{\text { S. }} \\
\text { S. } \\
\dot{.}\end{array}$ & $\begin{array}{rrr}-21 & 47 & 27.5 \\
-21 & 56 & 22.9 \\
-8 & 45 & 2.2 \\
-3 & 27 & 27.2 \\
. & \cdots & \cdots\end{array}$ & $\begin{array}{r}+2.3 \\
+2.3 \\
+3.5 \\
+3.1 \\
\ldots\end{array}$ & $\begin{array}{r}+2.0 \\
+1.2 \\
+1.9 \\
+1.9 \\
\quad \ldots\end{array}$ & $\begin{array}{cc}16 & 9.7 \\
\ldots & \ldots . . \\
10 & 59.4 \\
\ldots & \cdots . .\end{array}$ & $\begin{array}{r}-2.1 \\
\cdots \\
-1.6 \\
\cdots\end{array}$ \\
\hline & \begin{tabular}{l|l}
24.6 & \\
25.6 & \\
26.7 & \\
27.7 & \\
29.8 &
\end{tabular} & $\begin{array}{l}\mathrm{P} . \\
\text { M. } \\
\mathrm{Hi} . \\
\mathrm{P} . \\
\mathrm{M} .\end{array}$ & & $\begin{array}{l}\text { II } \\
\text { II } \\
\text { II } \\
\text { II } \\
\text { II }\end{array}$ & $\begin{array}{lll}2 & 52 & 22.66 \\
3 & 43 & 50.98 \\
4 & 35 & 45.46 \\
5 & 28 & 11.87 \\
7 & 13 & 54.66\end{array}$ & $\begin{array}{l}+0.25 \\
+0.12 \\
+0.10 \\
+0.24 \\
+0.24\end{array}$ & $\begin{array}{l}+0.07 \\
-0.07 \\
-0.12 \\
-0.02 \\
-0.11\end{array}$ & $\begin{array}{l}\ldots \ldots . \\
\cdots \cdots \\
\ldots \ldots . \\
\cdots \cdots\end{array}$ & \begin{tabular}{l|}
$\ldots .$. \\
$\cdots .$. \\
$\cdots .$. \\
$\cdots$
\end{tabular} & $\begin{array}{l}\text { N. } \\
\text { N. } \\
\text { N. } \\
\text { S. }\end{array}$ & $\begin{array}{rrrr}+11 & 40 & 13.1 \\
+15 & 37 & 9.1 \\
+18 & 43 & 42.1 \\
+20 & 53 & 3.1 \\
+22 & 4 & 44.1\end{array}$ & $\begin{array}{r}+1.8 \\
-0.7 \\
-0.1 \\
0.0 \\
+1.5\end{array}$ & $\begin{array}{l}+1.4 \\
-1.1 \\
-0.5 \\
-0.3 \\
+1.8\end{array}$ & $\begin{array}{cc}\ldots & \ldots . \\
\cdots & \cdots \\
\cdots & \cdots \\
\cdots & \cdots \\
\cdots & \cdots \\
\end{array}$ & $\begin{array}{l}\cdots \\
\cdots \\
\cdots \\
\cdots\end{array}$ \\
\hline Oct. & \begin{tabular}{r|}
30.8 \\
1.8 \\
10.1 \\
12.2 \\
13.2
\end{tabular} & $\begin{array}{l}\text { Hi. } \\
\text { P. } \\
\text { P. } \\
\text { Mi. }\end{array}$ & & $\begin{array}{l}\text { II } \\
\text { II } \\
\text { I } \\
\text { I } \\
\text { I }\end{array}$ & $\begin{array}{rrr}8 & 6 & 28.15 \\
8 & 58 & 22.08 \\
15 & 52 & 18.31 \\
17 & 53 & 51.87 \\
18 & 57 & 18.92\end{array}$ & $\begin{array}{l}+0.24 \\
+0.54 \\
+0.54 \\
+0.72 \\
+0.51\end{array}$ & $\begin{array}{l}-0.14 \\
+0.14 \\
+0.01 \\
+0.17 \\
-0.03\end{array}$ & $\begin{array}{l}\ldots \ldots . \\
\cdots \cdots \cdot \\
\cdots \cdots \cdot \\
\cdots \cdots\end{array}$ & $\begin{array}{l}\ldots . . \\
\cdots \cdots \\
\cdots \cdot . \\
\cdots \cdot\end{array}$ & $\dot{:}$. & 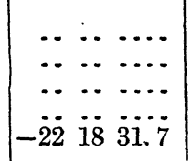 & $\begin{array}{r}\ldots \\
\ldots \\
+\ddot{4} .7\end{array}$ & $\begin{array}{r}\cdots \\
\cdots \\
\cdots \\
+3.8\end{array}$ & $\begin{array}{cc}\ldots & \cdots \\
\because & \cdots \\
\cdots & \cdots \\
\cdots & \cdots \\
\cdots & \cdots \\
.\end{array}$ & $\begin{array}{l}\cdots \\
\cdots \\
\cdots \\
\cdots\end{array}$ \\
\hline & $\begin{array}{l}14.3 \\
15.3 \\
16.4 \\
17.4 \\
18.4\end{array}$ & $\begin{array}{l}\text { M. } \\
\text { Hi. } \\
\text { P. } \\
\text { M. } \\
\text { Hi. }\end{array}$ & & $\begin{array}{l}I \\
I \\
I \\
I \\
I\end{array}$ & \begin{tabular}{rrr|}
20 & 0 & 42.61 \\
21 & 2 & 36.12 \\
22 & 2 & 4.16 \\
22 & 58 & 51.18 \\
23 & 53 & 15.81
\end{tabular} & $\begin{array}{l}+0.54 \\
+0.37 \\
+0.43 \\
+0.28 \\
+0.30\end{array}$ & $\begin{array}{l}+0.05 \\
-0.08 \\
+0.04 \\
-0.07 \\
-0.01\end{array}$ & $\cdots$ & \begin{tabular}{l|}
$\ldots .$. \\
$\cdots \cdots$ \\
$\cdots \cdots$ \\
$\cdots$
\end{tabular} & $\begin{array}{l}\text { S. } \\
\text { S. } \\
\text { S. } \\
\text { S. } \\
\text { S. }\end{array}$ & $\begin{array}{llll}-21 & 22 & 43.3 \\
-18 & 59 & 54.8 \\
-15 & 23 & 48.3 \\
-10 & 52 & 53.2 \\
-5 & 47 & 25.0\end{array}$ & $\begin{array}{l}+2.4 \\
+3.6 \\
+3.8 \\
+4.4 \\
+5.2\end{array}$ & $\begin{array}{l}+0.8 \\
+1.6 \\
+1.7 \\
+2.4 \\
+3.3\end{array}$ & $\begin{array}{cc}\ldots & \cdots \\
\because & \cdots \\
\cdots & \cdots \\
\cdots & \cdots \\
\cdots & \cdots \\
\end{array}$ & $\begin{array}{l}\cdots \\
\cdots \\
\cdots \\
\cdots\end{array}$ \\
\hline & $\begin{array}{l}19.5 \\
20.5 \\
21.5 \\
23.6 \\
24.6\end{array}$ & $\begin{array}{l}\text { P. } \\
\text { Hil. } \\
\text { Hi. } \\
\text { M. }\end{array}$ & E. & $\begin{array}{l}\text { I } \\
\text { İ } \\
\text { II } \\
\text { II }\end{array}$ & \begin{tabular}{rrr|}
0 & 45 & 55.60 \\
1 & 37 & 34.82 \\
2 & 28 & 55.89 \\
4 & 12 & 43.76 \\
5 & 5 & 34.26
\end{tabular} & $\begin{array}{l}+0.31 \\
+0.11 \\
+0.04 \\
+0.19 \\
+0.25\end{array}$ & $\begin{array}{l}+0.03 \\
-0.15 \\
-0.21 \\
-0.08 \\
-0.04\end{array}$ & $\begin{array}{l}\ddot{6} .95 \\
\cdots \cdots \\
\cdots \cdots \\
\cdots \cdots\end{array}$ & $\begin{array}{r}-0.10 \\
\ldots \ldots \\
\cdots \\
\cdots\end{array}$ & $\begin{array}{l}\text { S. } \\
\text { S. } \\
\dot{\text { N. }}\end{array}$ & 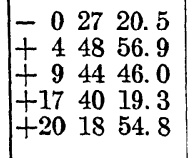 & $\begin{array}{l}+3.4 \\
+5.2 \\
+1.8 \\
-0.3 \\
-0.2\end{array}$ & $\begin{array}{l}+1.8 \\
+3.8 \\
+0.6 \\
-1.2 \\
-0.9\end{array}$ & $\begin{array}{cc}\cdots & \cdots \\
15 & 0.3 \\
15 & \cdots \\
15 & 0.2\end{array}$ & $\begin{array}{r}\cdots \\
-\ddot{1} . \ddot{8} \\
-\ddot{0.0}\end{array}$ \\
\hline
\end{tabular}




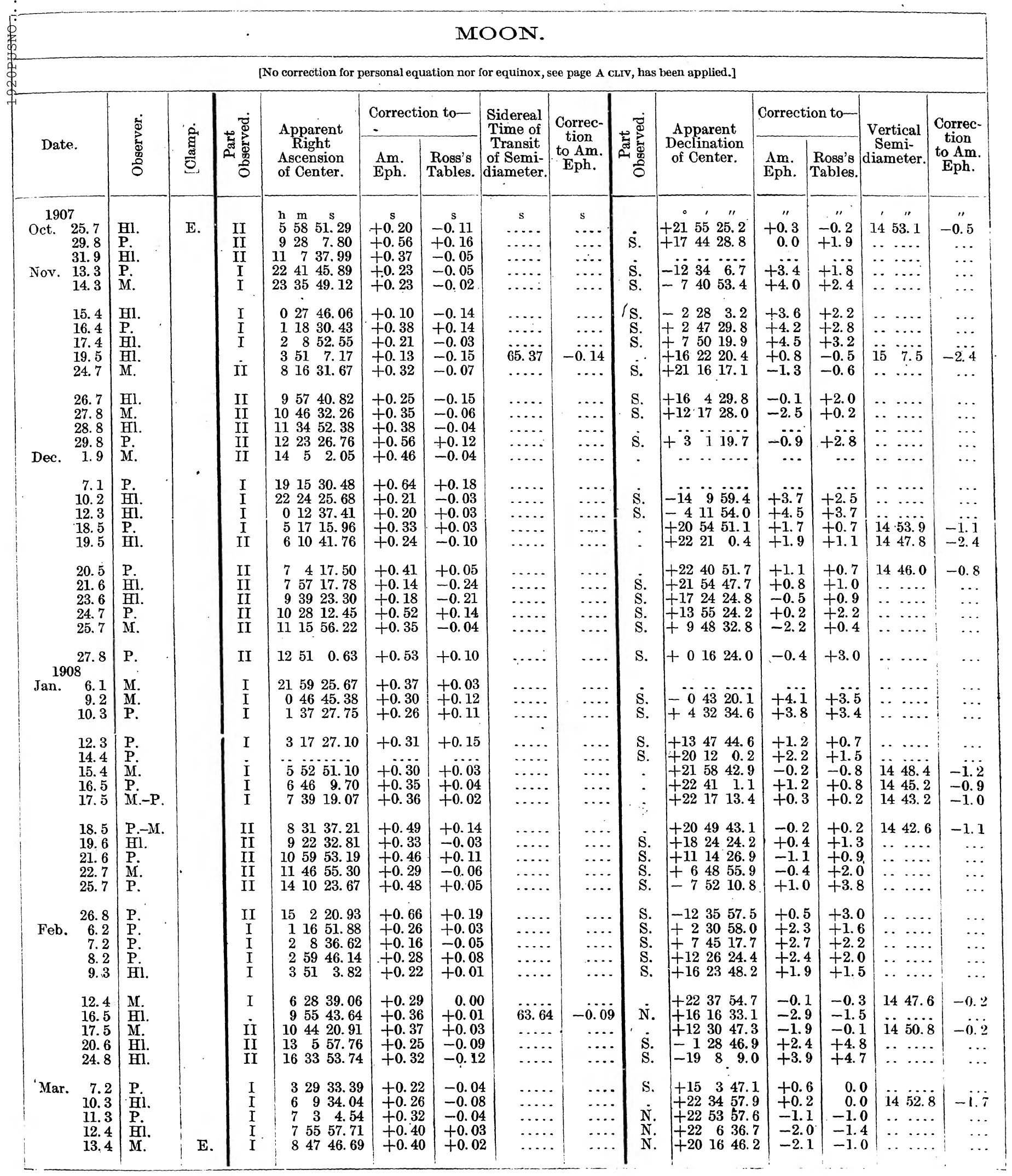




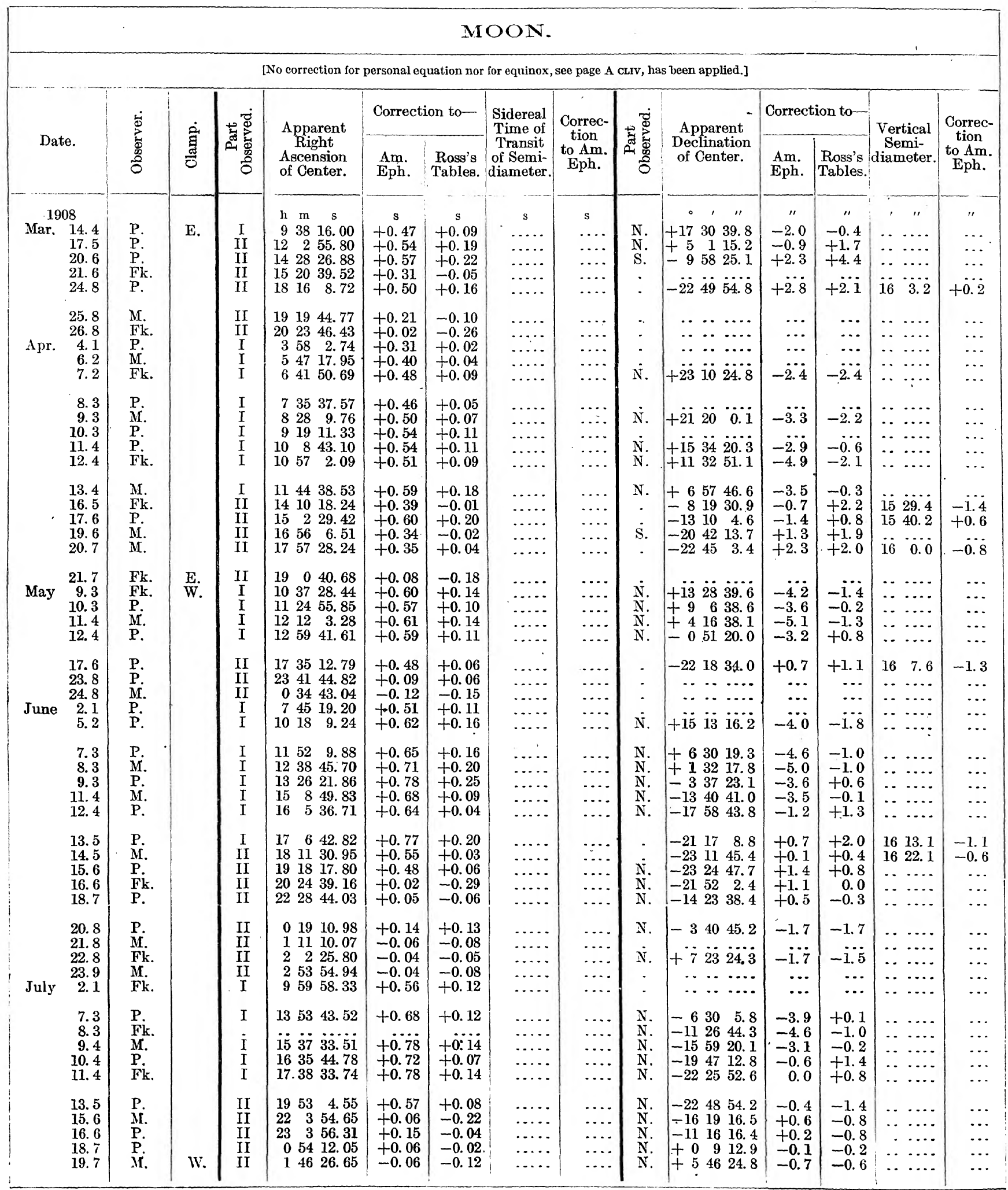




\section{MOON}

[No correction for personal equation nor for equinox, see paige A cLrv, has been applied.]

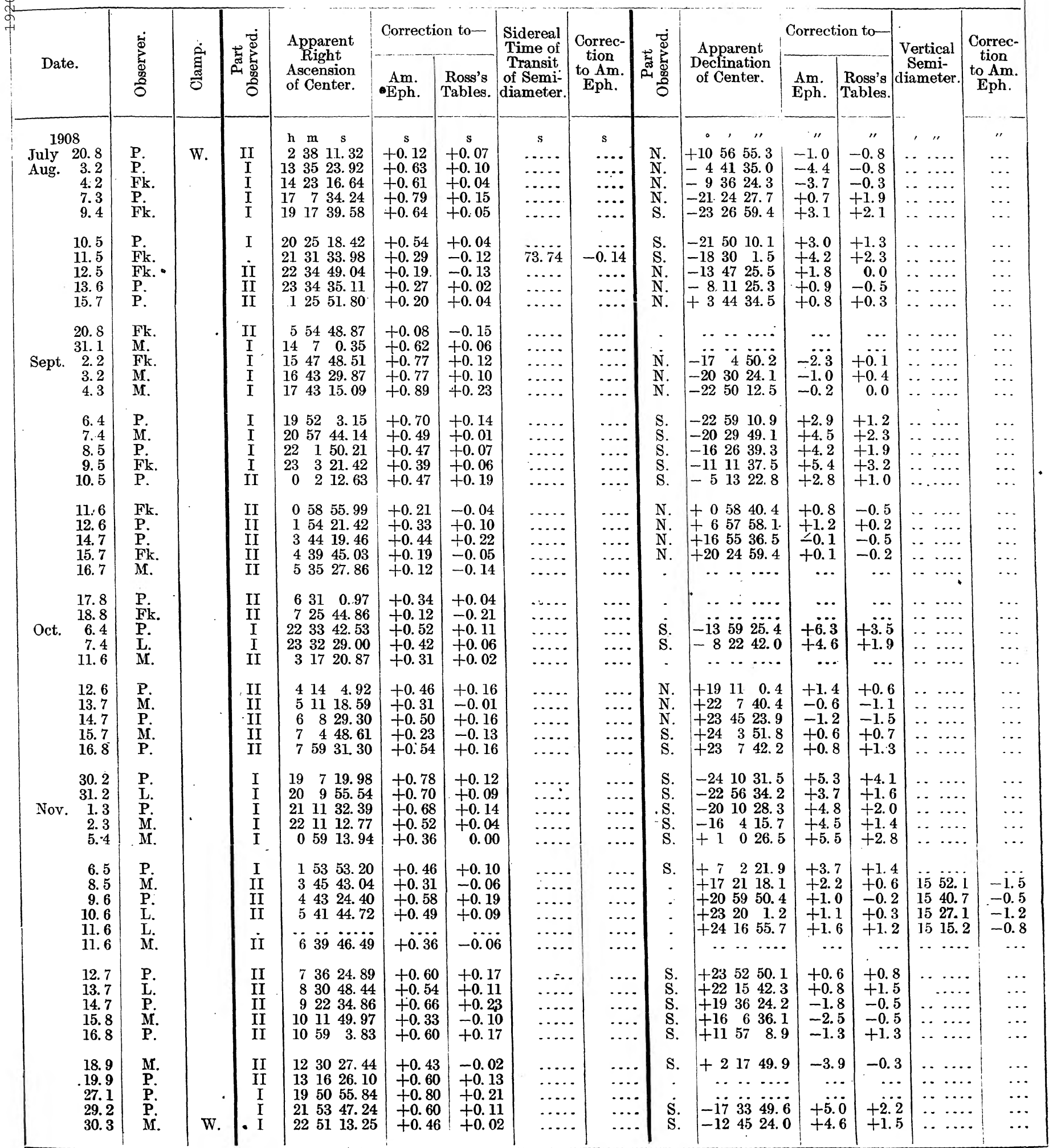




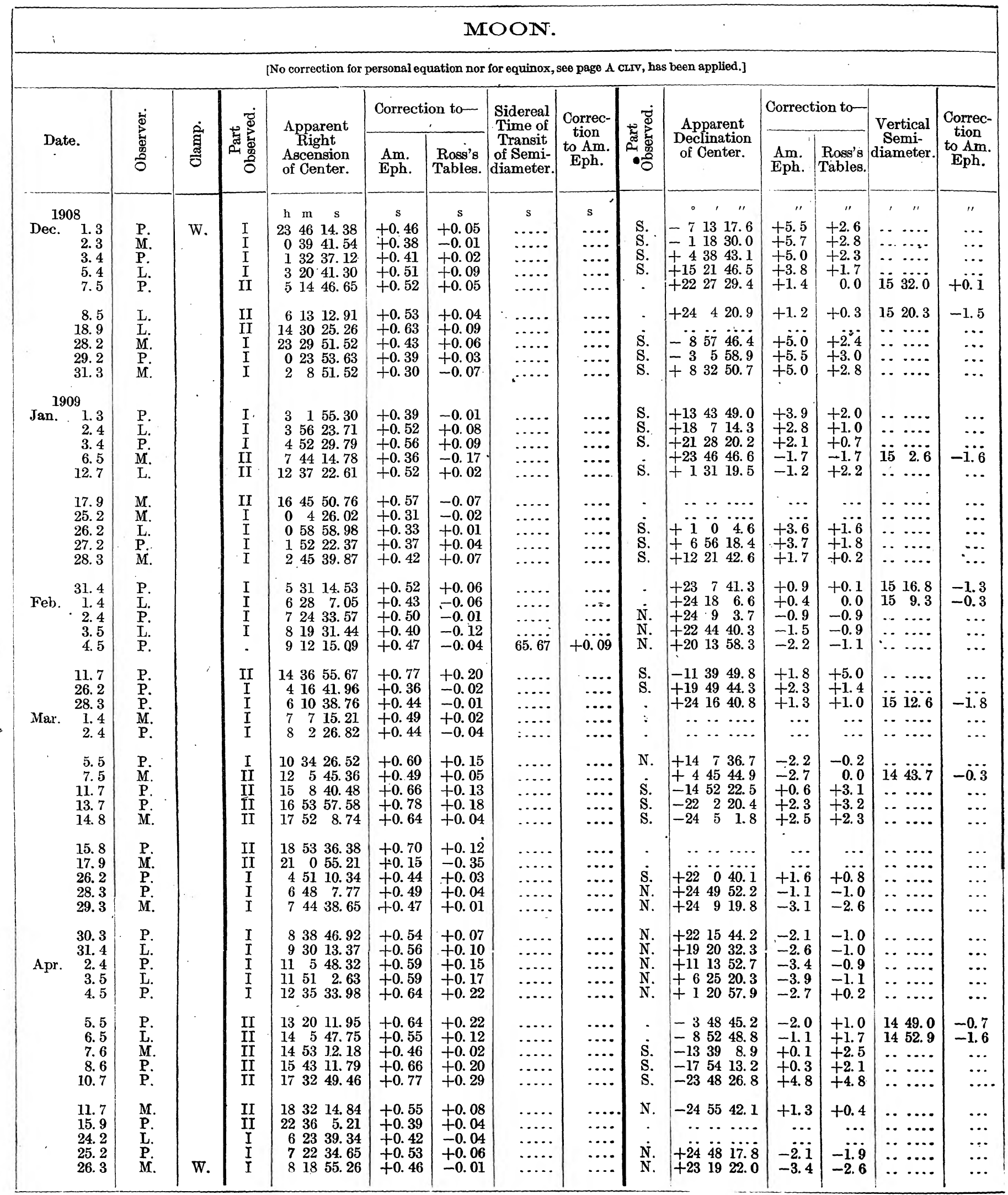




\section{MOON.}

[No correction for personal equation nor for equinox, see page A CLIV, has been applied.]

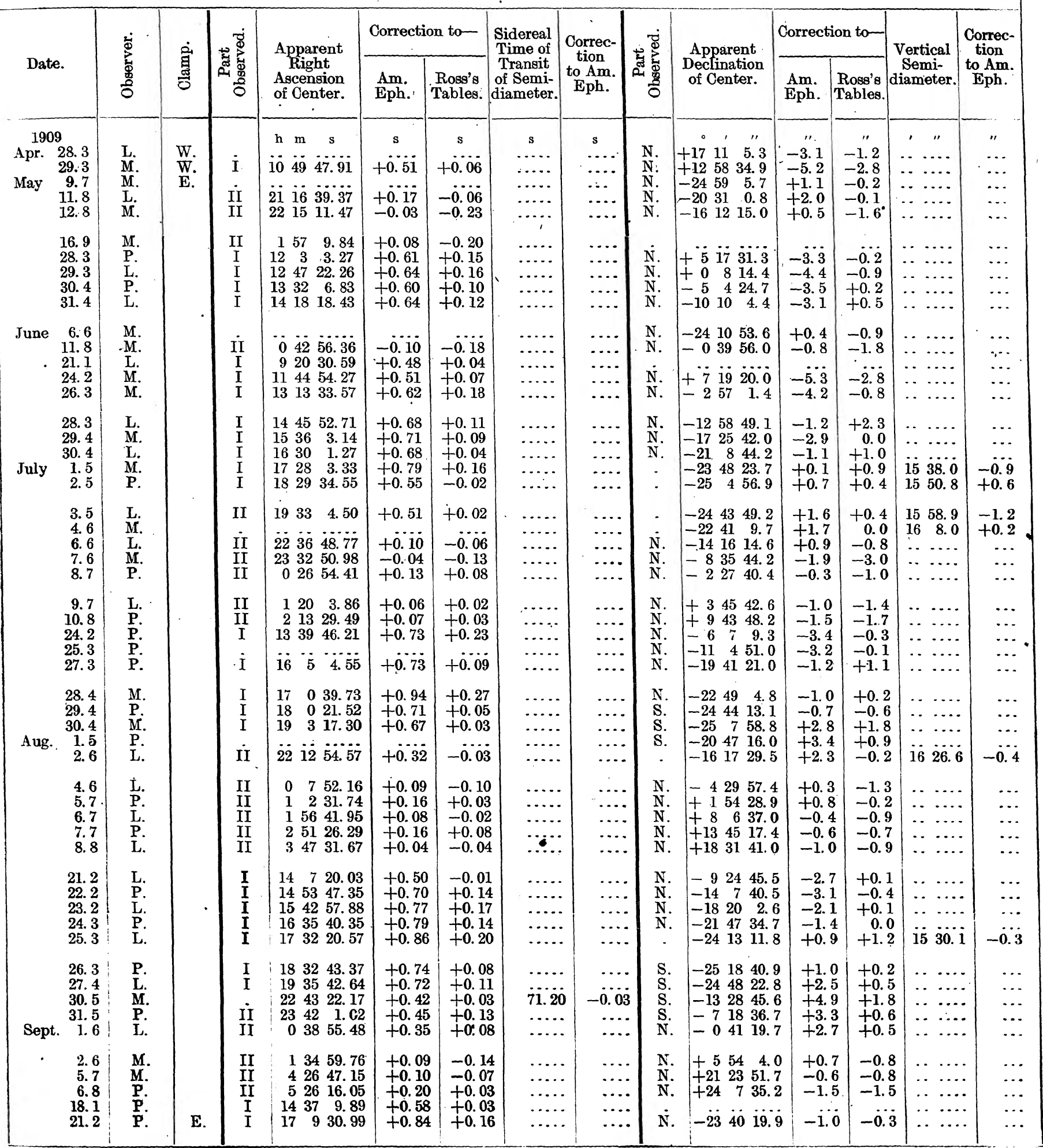




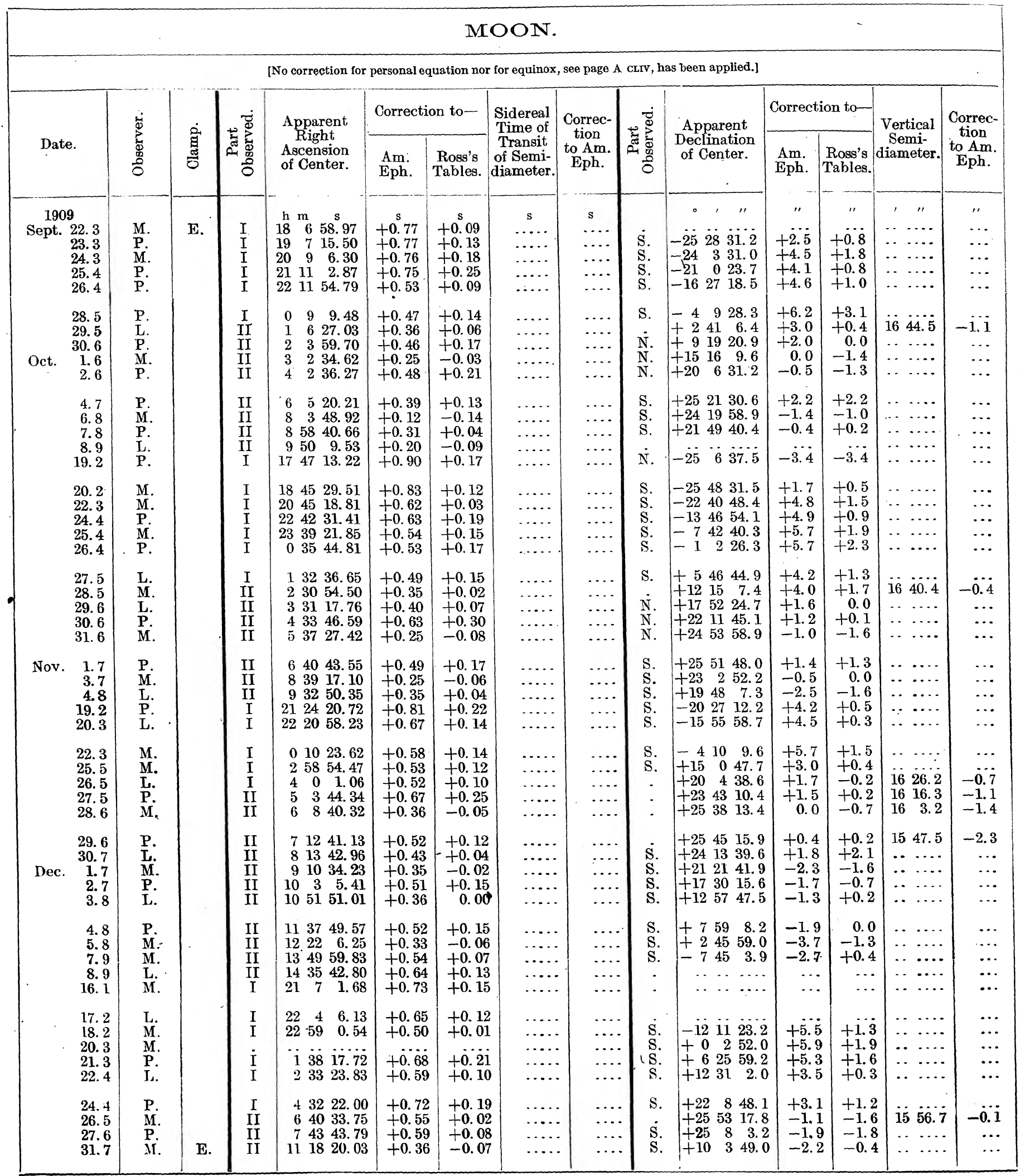




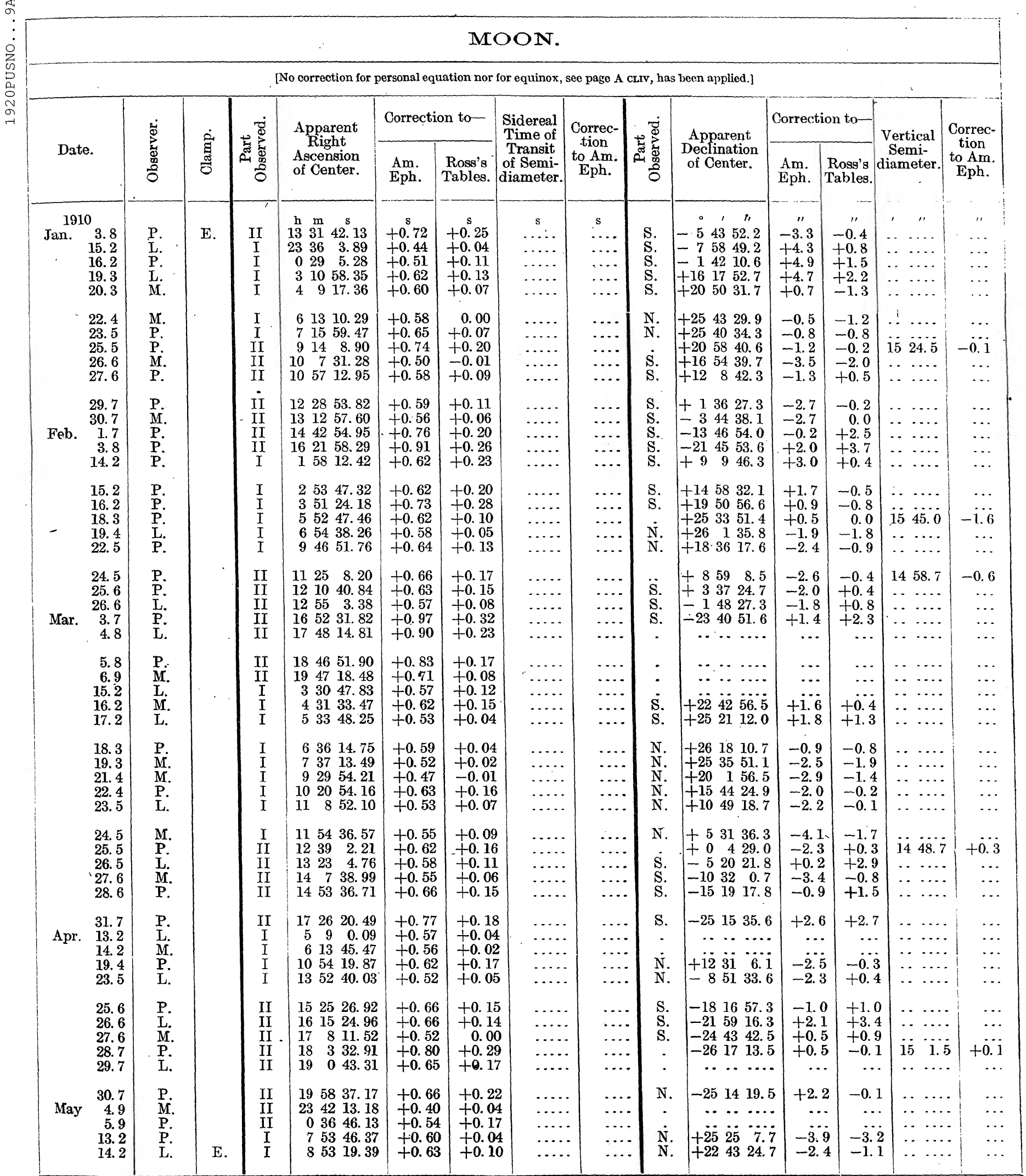




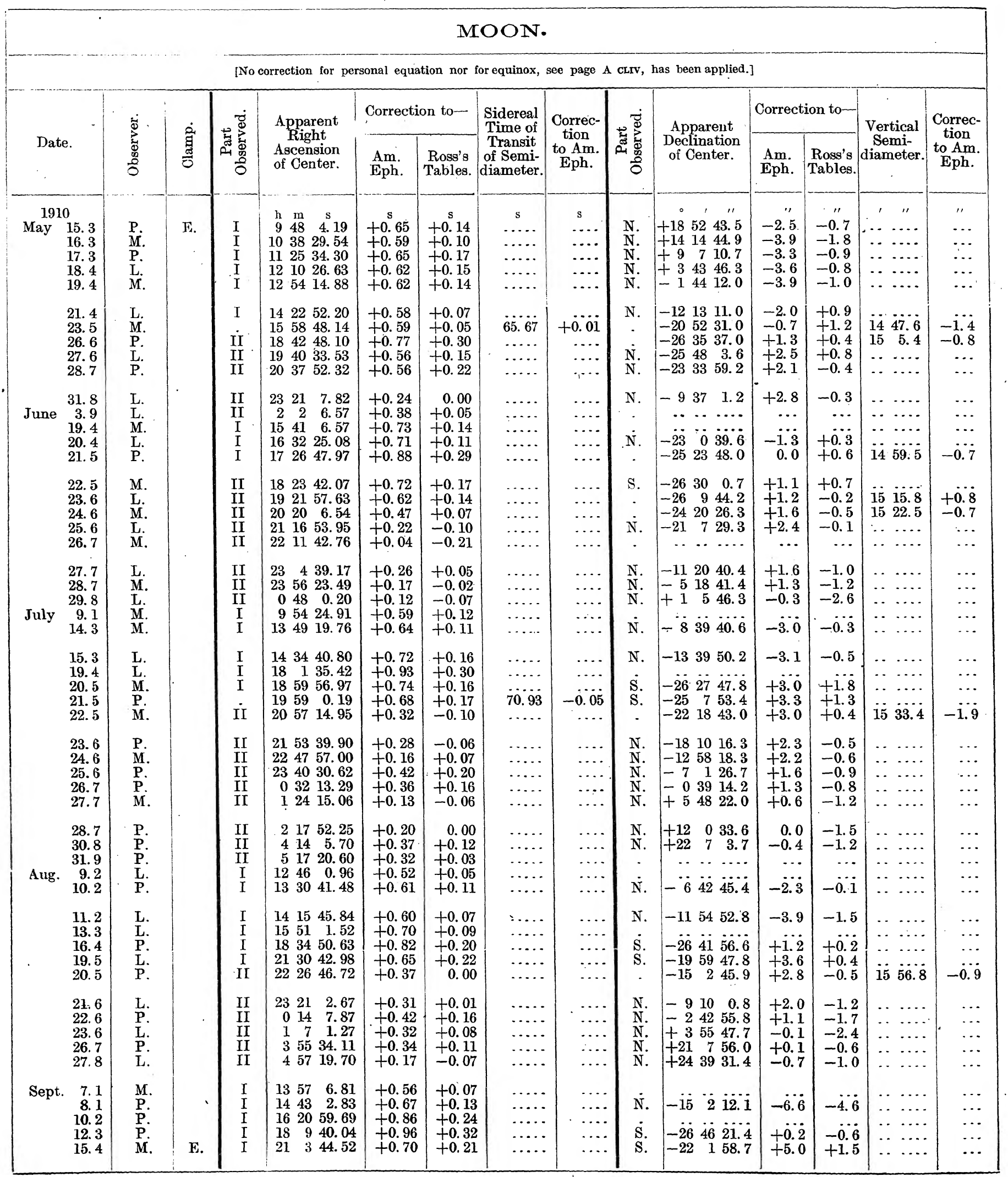


MOON.

[No correction for personal equation nor for equinox, see page A curv, has been applied.]

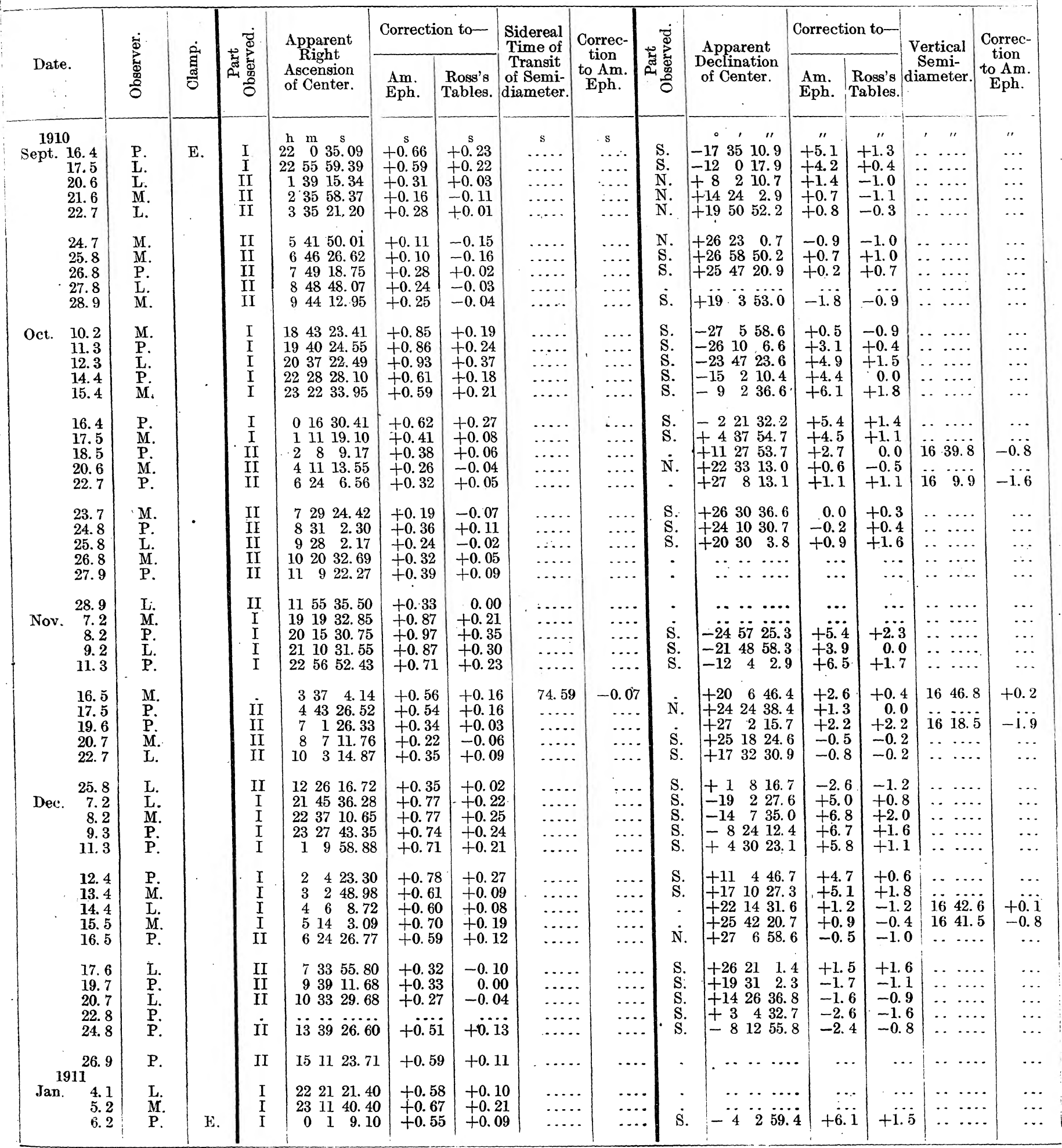




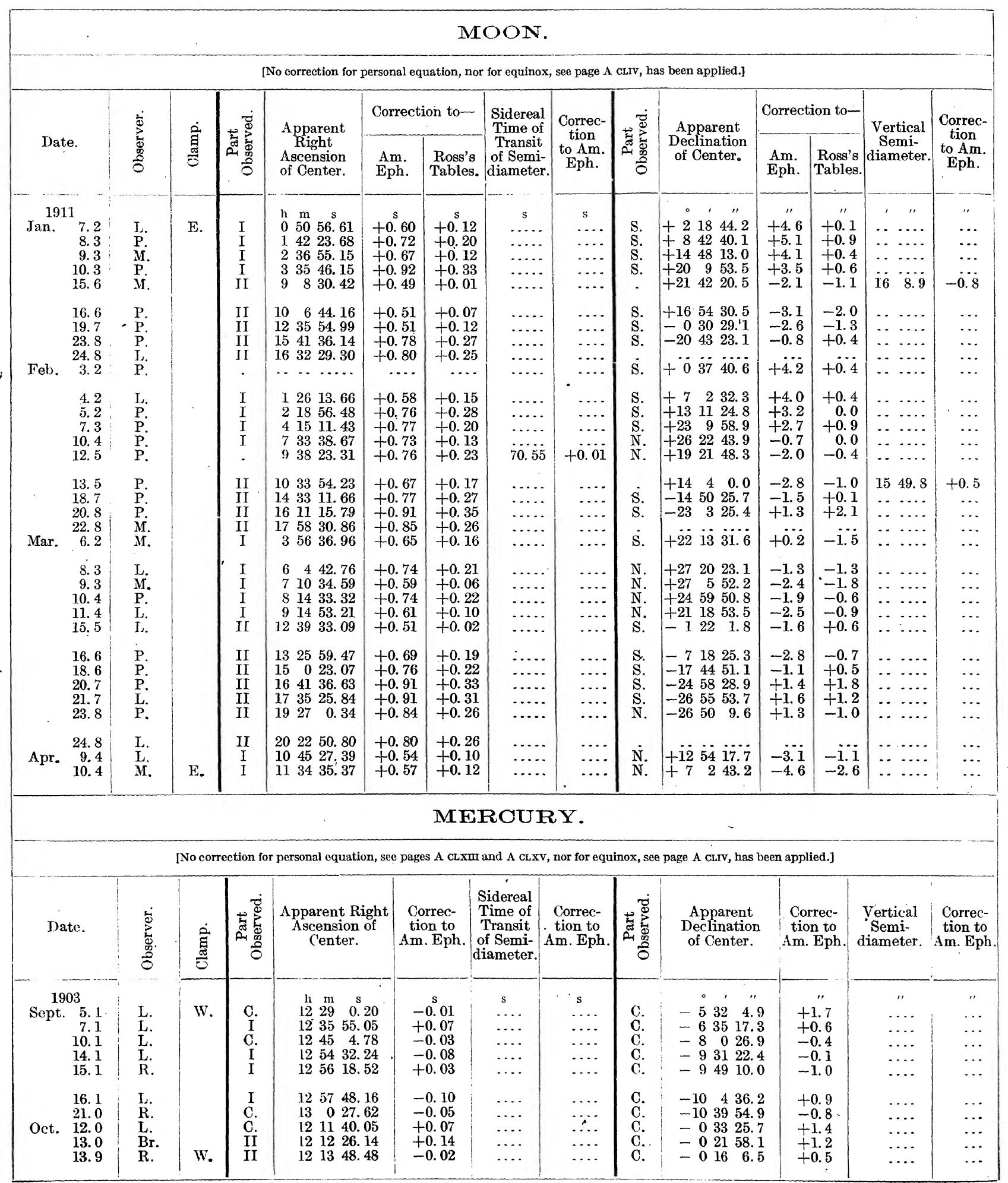




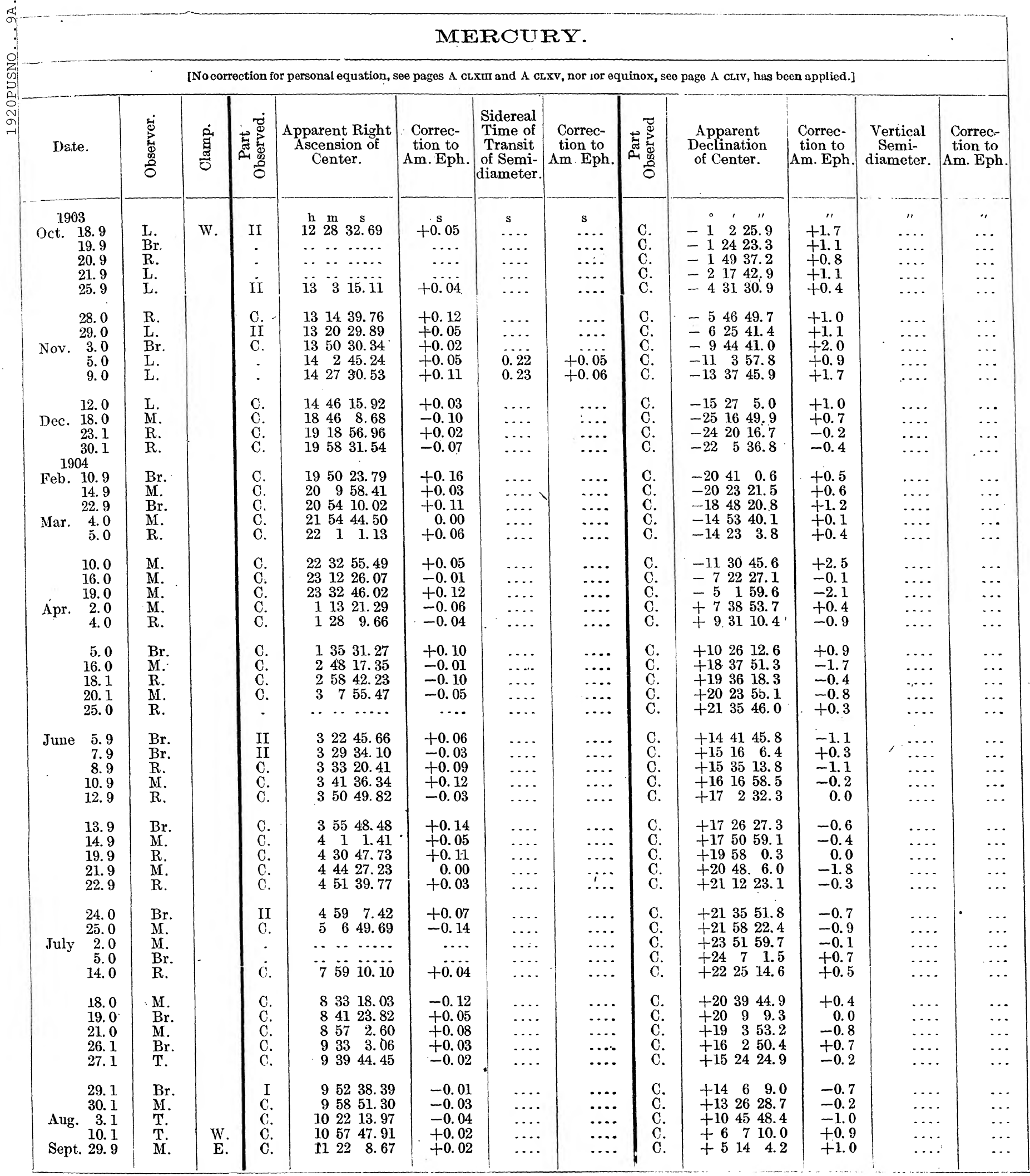




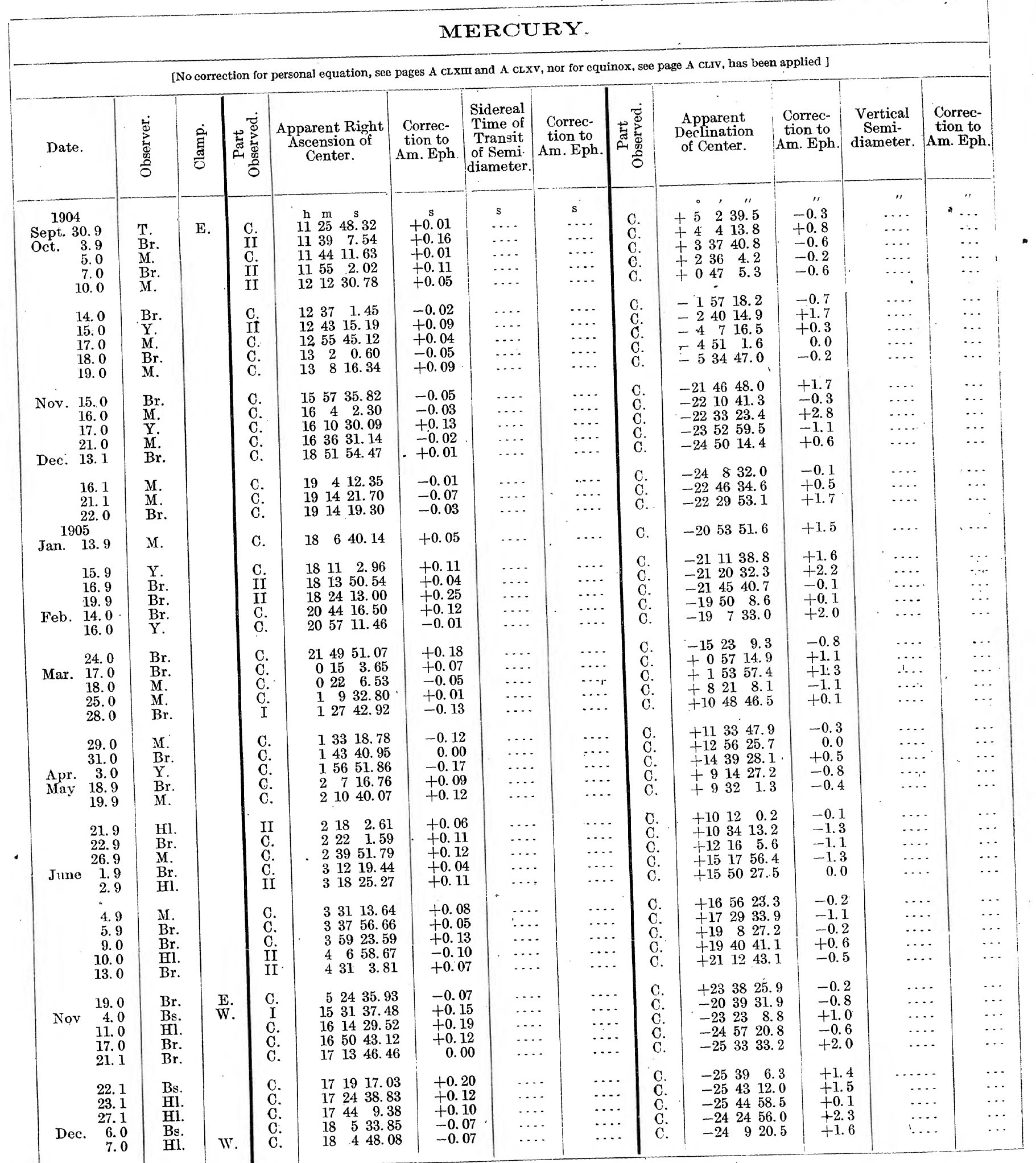




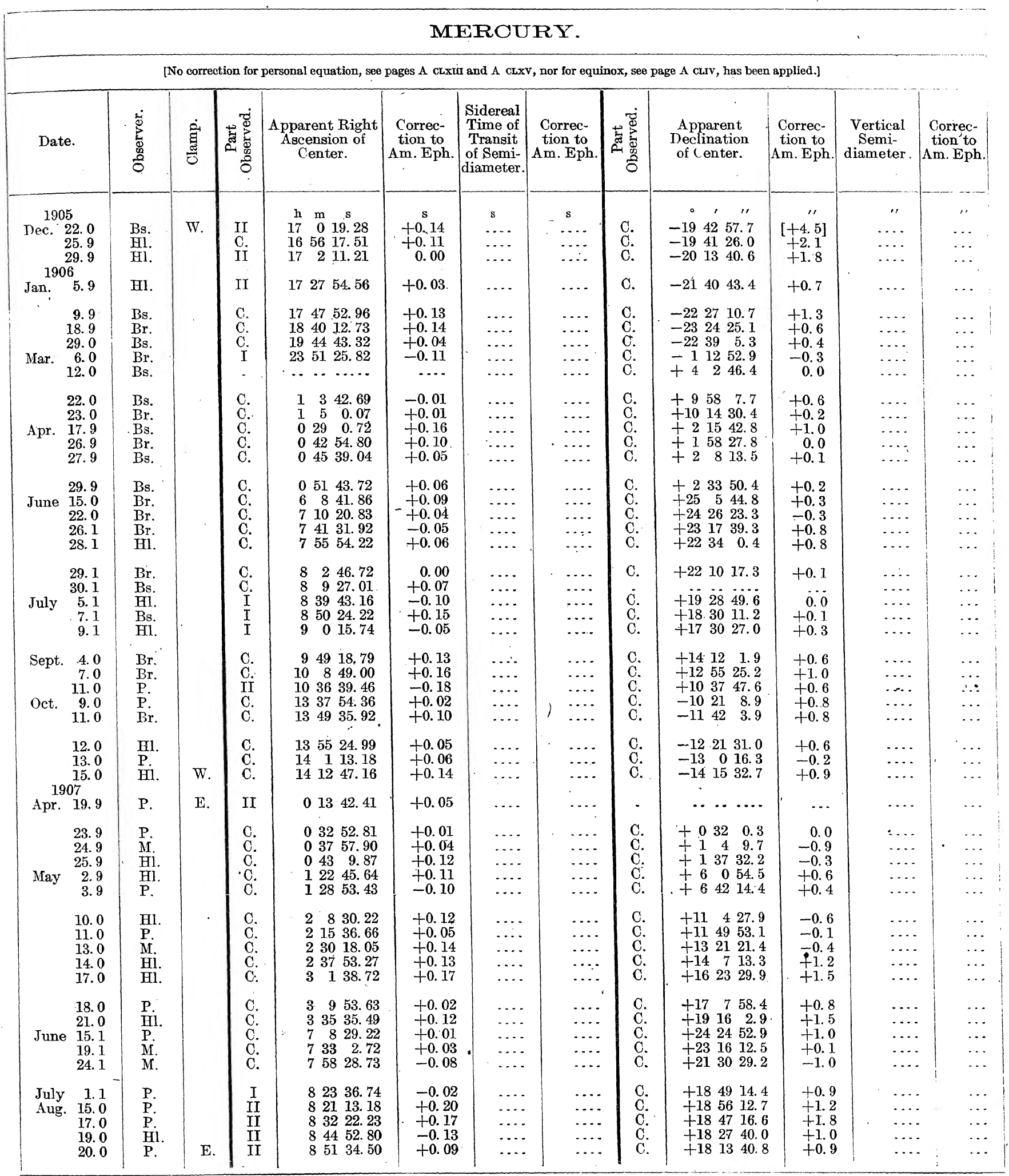




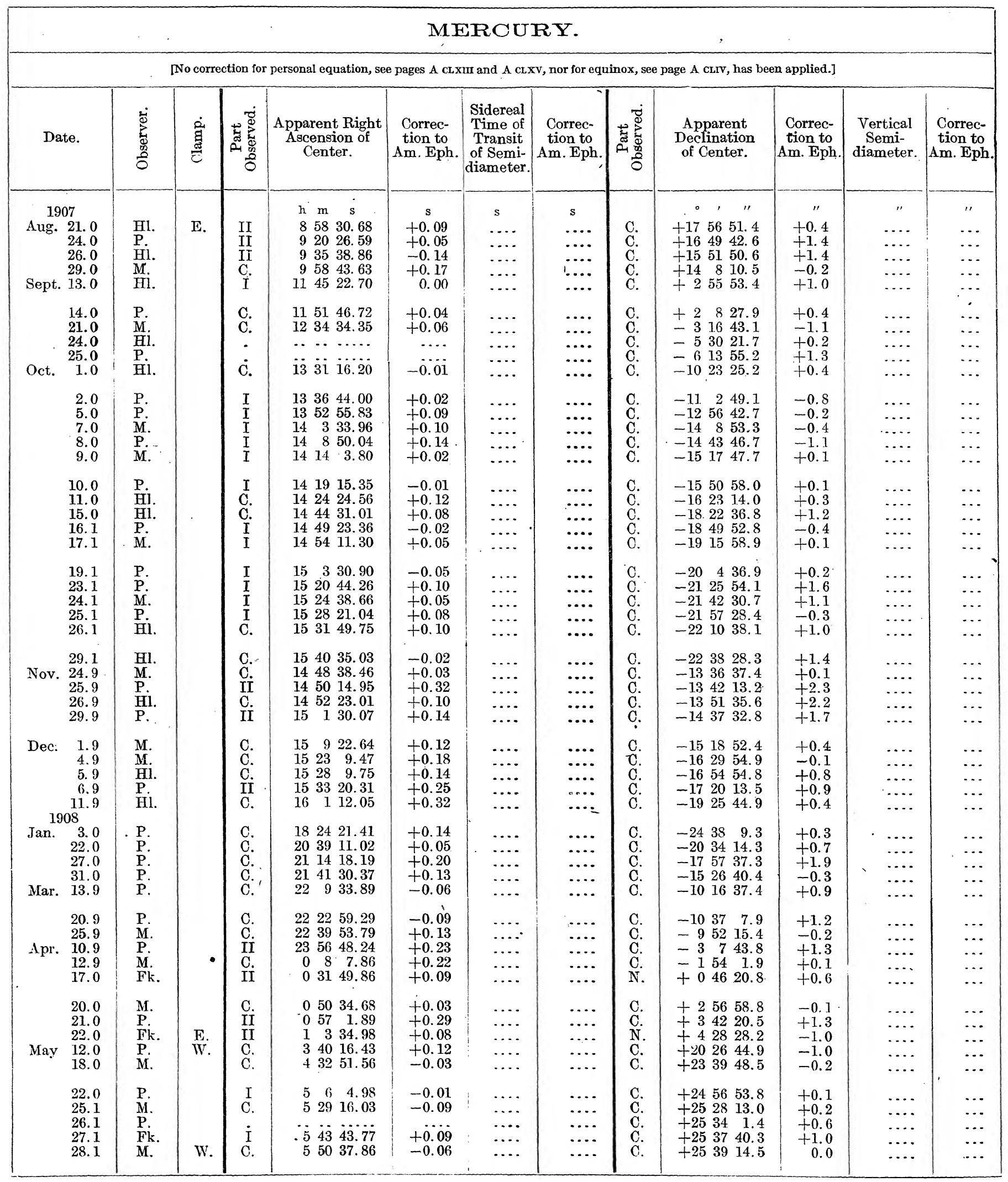




\section{MEROURY.}

\begin{tabular}{|c|c|c|c|c|c|c|c|c|c|c|c|c|}
\hline Date. & 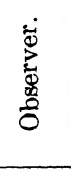 & 宊 & 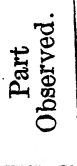 & $\begin{array}{l}\text { Apparent Right } \\
\text { Ascension of } \\
\text { Center. }\end{array}$ & $\begin{array}{c}\text { Correc- } \\
\text { tion to } \\
\text { Am. Eph. }\end{array}$ & $\begin{array}{l}\text { Sidereal } \\
\text { Time of } \\
\text { Transit } \\
\text { of Semi- } \\
\text { diameter. }\end{array}$ & $\begin{array}{c}\text { Correc- } \\
\text { tion to } \\
\text { Am. Eph. }\end{array}$ & 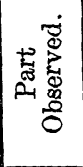 & $\begin{array}{c}\text { Apparent } \\
\text { Declination } \\
\text { of Center. }\end{array}$ & $\begin{array}{c}\text { Correc- } \\
\text { tion to } \\
\text { Am. Eph. }\end{array}$ & $\begin{array}{c}\text { Vertical } \\
\text { Semi- } \\
\text { diameter. }\end{array}$ & $\begin{array}{c}\text { Correc- } \\
\text { tion to } \\
\text { Am. Eph. }\end{array}$ \\
\hline \begin{tabular}{cc}
\multicolumn{2}{c}{1908} \\
June & 1.1 \\
2.1 \\
5.1 \\
8.1 \\
9.1
\end{tabular} & $\begin{array}{l}\text { Fk. } \\
\text { P. } \\
\text { P. } \\
\text { M. } \\
\text { P. }\end{array}$ & W. & $\begin{array}{l}\text { I } \\
\text { I } \\
\text { I } \\
\text { I } \\
\text { I }\end{array}$ & $\begin{array}{lcc}\mathrm{h} & \mathrm{m} & \mathrm{s} \\
6 & 15 & 55.10 \\
6 & 21 & 37.64 \\
6 & 37 & 11.90 \\
6 & 50 & 20.11 \\
6 & 54 & 8.77\end{array}$ & $\begin{array}{c}\mathrm{s} \\
-0.04 \\
-0.07 \\
-0.22 \\
0.00 \\
-0.03\end{array}$ & $\begin{array}{l}\mathrm{s} \\
\cdots \\
\cdots \\
\cdots \\
\cdots \\
\cdots\end{array}$ & $\begin{array}{l}\mathrm{s} \\
\cdots \\
\cdots \\
\cdots \\
\cdots \\
\cdots\end{array}$ & $\begin{array}{l}\text { C. } \\
\text { C. } \\
\text { C. } \\
\text { C. } \\
\text { C. }\end{array}$ & $\begin{array}{rrr}\circ & \prime \prime \\
+25 & 27 & 13.8 \\
+25 & 20 & 10.6 \\
+24 & 51 & 2.6 \\
+24 & 12 & 12.7 \\
+23 & 57 & 35.7\end{array}$ & $\begin{array}{l}11 \\
+0.9 \\
+0.7 \\
-0.8 \\
-0.4 \\
+0.3\end{array}$ & $\begin{array}{l}\text { " } \\
\cdots \\
\cdots \cdots \\
\cdots \\
\cdots \\
\cdots\end{array}$ & $\begin{array}{l}\prime \\
\cdots \\
\cdots \\
\cdots \\
\cdots\end{array}$ \\
\hline $\begin{array}{ll} & 12.1 \\
16.1 \\
\text { July } \\
16.0 \\
17.0 \\
19.9\end{array}$ & $\begin{array}{l}\text { P. } \\
\text { P. } \\
\text { P. } \\
\text { M. }\end{array}$ & ' & $\begin{array}{l}\text { I } \\
\text { I } \\
\text { II } \\
\text { II } \\
\text { C. }\end{array}$ & $\begin{array}{rrr}7 & 3 & 48.25 \\
7 & 12 & 21.22 \\
6 & 34 & 33.59 \\
6 & 35 & 2.96 \\
6 & 38 & 54.79\end{array}$ & $\begin{array}{l}-0.12 \\
-0.03 \\
-0.04 \\
+0.13 \\
-0.03\end{array}$ & $\begin{array}{l}\cdots \\
\cdots \\
\cdots \\
\cdots\end{array}$ & $\begin{array}{l}\cdots \\
\cdots \\
\cdots \\
\cdots \cdots\end{array}$ & $\begin{array}{l}\text { C. } \\
\text { C. } \\
\text { C. } \\
\text { C. } \\
\text { C. }\end{array}$ & $\begin{array}{rrr}+23 & 10 & 4.0 \\
+22 & 1 & 50.1 \\
+19 & 2 & 23.8 \\
+19 & 12 & 34.8 \\
+19 & 45 & 42.5\end{array}$ & $\begin{array}{l}-0.3 \\
-0.7 \\
+0.3 \\
+0.8 \\
-0.5\end{array}$ & $\begin{array}{l}\cdots \\
\cdots \\
\cdots \\
\cdots \\
\cdots\end{array}$ & $\begin{array}{l}\cdots \\
\cdots \\
\cdots \\
\cdots\end{array}$ \\
\hline $\begin{array}{r}28.9 \\
\text { Aug. } 3.0 \\
4.0 \\
5.0 \\
10.0\end{array}$ & $\begin{array}{l}\text { P. } \\
\text { P. } \\
\text { Fk. } \\
\text { P. } \\
\text { P. }\end{array}$ & & $\begin{array}{l}\text { II } \\
\text { II } \\
\text { II } \\
\text { II } \\
\text { II }\end{array}$ & $\begin{array}{rrr}7 & 12 & 32.92 \\
7 & 44 & 21.37 \\
7 & 51 & 37.14 \\
7 & 59 & 7.84 \\
8 & 39 & 14.89\end{array}$ & $\begin{array}{l}+0.17 \\
+0.16 \\
+0.01 \\
+0.17 \\
+0.25\end{array}$ & $\begin{array}{l}\cdots \\
\cdots \\
\cdots \\
\cdots\end{array}$ & $\begin{array}{l}\cdots \\
\cdots \\
\cdots \\
\cdots\end{array}$ & $\begin{array}{l}\text { C. } \\
\text { C. } \\
\text { C. } \\
\text { C. } \\
\text { C. }\end{array}$ & $\begin{array}{rrr}+21 & 11 & 59.2 \\
+21 & 15 & 23.4 \\
+21 & 9 & 30.6 \\
+21 & 1 & 8.0 \\
+19 & 39 & 32.2\end{array}$ & $\begin{array}{l}+0.4 \\
+1.3 \\
+0.4 \\
+0.2 \\
-1.0\end{array}$ & $\begin{array}{l}\cdots \\
\cdots \\
\cdots \\
\cdots \\
\cdots\end{array}$ & $\begin{array}{l}\cdots \\
\cdots \\
\cdots \\
\cdots\end{array}$ \\
\hline $\begin{array}{l}11.0 \\
12.0 \\
13.0 \\
15.0 \\
31.0\end{array}$ & $\begin{array}{l}\text { Fk. } \\
\text { Fk. } \\
\text { Fk. } \\
\text { Fk. } \\
\text { M. }\end{array}$ & & $\begin{array}{l}\text { C. } \\
\text { C. } \\
\text { C. } \\
\text { C. }\end{array}$ & $\begin{array}{rrr}8 & 47 & 33.37 \\
8 & 55 & 53.31 \\
9 & 4 & 12.87 \\
9 & 20 & 45.57 \\
11 & 18 & 2.01\end{array}$ & $\begin{array}{l}+0.13 \\
+0.09 \\
-0.03 \\
+0.07 \\
-0.04\end{array}$ & $\begin{array}{l}\cdots \\
\cdots \\
\cdots \\
\cdots \\
\cdots\end{array}$ & $\begin{array}{l}\cdots \\
\cdots \\
\cdots \\
\cdots \\
\cdots\end{array}$ & $\begin{array}{l}\text { C. } \\
\text { C. } \\
\text { C. } \\
\text { C. } \\
\text { C. }\end{array}$ & $\begin{array}{r}+191520.7 \\
+184839.1 \\
+181935.7 \\
+1715 \quad 1.8 \\
+54633.1\end{array}$ & $\begin{array}{l}+0.7 \\
+0.6 \\
+0.2 \\
+0.7 \\
-0.9\end{array}$ & $\begin{array}{l}\cdots \\
\cdots \\
\cdots \\
\cdots \\
\cdots\end{array}$ & $\begin{array}{l}\cdots \\
\cdots \\
\cdots \\
\cdots\end{array}$ \\
\hline 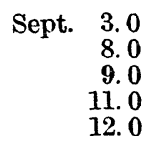 & $\begin{array}{l}\mathrm{M} . \\
\mathbf{P} \\
\mathrm{Fk} . \\
\mathrm{P} . \\
\mathrm{Fk} .\end{array}$ & & $\begin{array}{l}\text { C. } \\
\text { I } \\
\text { I } \\
\text { I } \\
\text { I }\end{array}$ & $\begin{array}{rrr}11 & 36 & 48.73 \\
12 & 6 & 22.09 \\
12 & 12 & 3.06 \\
12 & 23 & 12.30 \\
12 & 28 & 41.23\end{array}$ & $\begin{array}{l}-0.09 \\
-0.02 \\
+0.07 \\
-0.07 \\
+0.03\end{array}$ & $\begin{array}{l}\cdots \\
\cdots \\
\cdots \\
\cdots \\
\cdots\end{array}$ & $\begin{array}{l}\cdots \\
\cdots \\
\cdots \\
\cdots \\
\cdots\end{array}$ & $\begin{array}{l}\text { C. } \\
\text { C. } \\
\text { c. } \\
\text { c. } \\
\text { C. }\end{array}$ & $\begin{array}{rrr}+32652.7 \\
-022 & 6.0 \\
-1 & 657.4 \\
-2 & 3529.5 \\
-319 & 5.8\end{array}$ & $\begin{array}{l}-0.2 \\
-0.2 \\
+0.2 \\
-0.2 \\
-0.5\end{array}$ & $\begin{array}{l}\cdots \\
\cdots \\
\cdots \\
\cdots \\
\cdots\end{array}$ & $\begin{array}{l}\cdots \\
\cdots \\
\cdots \\
\cdots\end{array}$ \\
\hline $\begin{array}{rr} & 15.0 \\
& 18.0 \\
22.1 \\
\text { Oct. } \quad 7.1 \\
& 8.1\end{array}$ & $\begin{array}{l}\mathbf{P}: \\
\mathbf{P} . \\
\mathbf{P} . \\
\mathrm{M}\end{array}$ & & $\begin{array}{l}\text { I } \\
\text { I } \\
\text { I } \\
\text { I } \\
\text { I }\end{array}$ & $\begin{array}{rrr}12 & 44 & 45.50 \\
13 & 0 & 18.34 \\
13 & 20 & 14.09 \\
14 & 23 & 22.69 \\
14 & 26 & 29.09\end{array}$ & $\begin{array}{l}-0.07 \\
-0.11 \\
+0.04 \\
+0.07 \\
+0.08\end{array}$ & \begin{tabular}{l|}
$\cdots$ \\
$\cdots$ \\
$\cdots$ \\
$\cdots$ \\
$\cdots$
\end{tabular} & $\begin{array}{l}\cdots \\
\cdots \\
\cdots \\
\cdots\end{array}$ & $\begin{array}{l}\text { C. } \\
\text { C. } \\
\text { c. } \\
\text { C. }\end{array}$ & $\begin{array}{rrr}-5 & 26 & 55.6 \\
-7 & 29 & 43.2 \\
-10 & 4 & 18.1 \\
-17 & 30 & 50.2 \\
-17.50 \quad 0.5\end{array}$ & $\begin{array}{l}-0.1 \\
-0.4 \\
-0.2 \\
+0.8 \\
-1.2\end{array}$ & $\begin{array}{l}\cdots \\
\cdots \\
\cdots \\
\cdots \\
\cdots\end{array}$ & $\begin{array}{l}\cdots \\
\cdots \\
\cdots \\
\cdots\end{array}$ \\
\hline $\begin{array}{r}13.0 \\
15.0 \\
\text { Nov. } \\
11.9 \\
12.9\end{array}$ & $\begin{array}{l}\mathrm{P} . \\
\mathrm{P} . \\
\mathrm{P} . \\
\mathrm{M} .\end{array}$ & & $\begin{array}{l}\text { I } \\
\text { I } \\
\text { C. } \\
\text { C. } \\
\text { II }\end{array}$ & $\begin{array}{rrr}14 & 38 & 5.05 \\
14 & 40 & 20.74 \\
13 & 46 & 6.25 \\
13 & 57 & 51.49 \\
14 & 1 & 27.58\end{array}$ & $\begin{array}{l}-0.02 \\
-0.06 \\
+0.08 \\
+0.10 \\
+0.26\end{array}$ & $\begin{array}{l}\cdots \\
\cdots \\
\cdots \\
\cdots \\
\cdots\end{array}$ & $\begin{array}{l}\cdots \\
\cdots \\
\cdots \\
\cdots\end{array}$ & $\begin{array}{l}\text { C. } \\
\text { C. } \\
\text { C. } \\
\text { C. } \\
\text { C. }\end{array}$ & $\begin{array}{rrr}-18 & 54 & 44.5 \\
-19 & 249.0 \\
-9 & 0 & 36.3 \\
-9 & 33 & 56.3 \\
-9 & 53 & 19.7\end{array}$ & $\begin{array}{r}-0.7 \\
+0.6 \\
+0.4 \\
+0.2 \\
0.0\end{array}$ & $\begin{array}{l}\cdots \\
\cdots \\
\cdots \\
\cdots \\
\cdots\end{array}$ & $\begin{array}{l}\cdots \\
\cdots \\
\cdots \\
\cdots\end{array}$ \\
\hline $\begin{array}{l}15.9 \\
16.9 \\
17.9 \\
18.9 \\
19.9\end{array}$ & $\begin{array}{l}\mathrm{M} . \\
\mathrm{P} . \\
\mathrm{L} . \\
\mathrm{M} . \\
\mathrm{P} .\end{array}$ & & $\begin{array}{l}\text { C. } \\
\text { II } \\
\text { C. } \\
\text { C. } \\
\text { II }\end{array}$ & $\begin{array}{rrr}14 & 14 & 8.82 \\
14 & 18 & 53.33 \\
14 & 23 & 49.76 \\
14 & 28 & 56.80 \\
14 & 34 & 13.59\end{array}$ & $\begin{array}{l}+0.08 \\
+0.26 \\
+0.18 \\
+0.04 \\
+0.29\end{array}$ & $\begin{array}{l}\cdots \\
\cdots \\
\cdots \\
\cdots \\
\cdots\end{array}$ & $\begin{array}{l}\cdots \\
\cdots \\
\cdots \\
\cdots \\
\cdots\end{array}$ & $\begin{array}{l}\text { C. } \\
\text { C. } \\
\text { C. } \\
\text { C. } \\
\text { C. }\end{array}$ & $\begin{array}{rrr}-11 & 6 & 8.7 \\
-11 & 34 & 0.4 \\
-12 & 3 & 8.6 \\
-12 & 33 & 13.1 \\
-13 & 4 & 1.9\end{array}$ & $\begin{array}{l}+0.4 \\
+1.1 \\
-0.2 \\
+0.1 \\
-0.7\end{array}$ & $\begin{array}{l}\cdots \\
\cdots \\
\cdots \\
\cdots \\
\cdots\end{array}$ & $\begin{array}{l}\cdots \\
\cdots \\
\cdots \\
\cdots \\
\cdots\end{array}$ \\
\hline 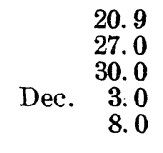 & $\begin{array}{l}\text { L. } \\
\text { P. } \\
\text { P. } \\
\text { P. }\end{array}$ & & $\begin{array}{l}\text { II } \\
\text { II } \\
\text { II } \\
\text { II } \\
\text { II }\end{array}$ & $\begin{array}{rrrr}14 & 39 & 38.27 \\
15 & 14 & 14.69 \\
15 & 38 & 43.89 \\
15 & 51 & 17: 08 \\
16 & 23 & 26.36\end{array}$ & $\begin{array}{l}+0.20 \\
+0.25 \\
+0.28 \\
+0.22 \\
+0.14\end{array}$ & $\begin{array}{l}\cdots \\
\cdots \\
\cdots \\
\cdots \\
\cdots\end{array}$ & $\begin{array}{l}\cdots \\
\cdots \\
\cdots \\
\cdots\end{array}$ & $\begin{array}{l}\text { C. } \\
\text { C. } \\
\text { C. } \\
\text { C. }\end{array}$ & $\begin{array}{rrr}-13 & 35 & 17.9 \\
-16 & 44 & 6.6 \\
-18 & 42 & 26.0 \\
-19 & 37 & 27.1 \\
-21 & 39 & 38.0\end{array}$ & $\begin{array}{r}+1.1 \\
+0.6 \\
0.0 \\
-0.8 \\
+0.3\end{array}$ & $\begin{array}{l}\cdots \\
\cdots \\
\cdots \\
\cdots \\
\cdots\end{array}$ & $\begin{array}{l}\cdots \\
\cdots \\
\cdots \\
\cdots\end{array}$ \\
\hline $\begin{array}{r}9.0 \\
31.0 \\
1909\end{array}$ & $\begin{array}{l}\mathbf{L} . \\
\mathbf{M}\end{array}$ & . & C. & $\begin{array}{rrr}16 & 29 & 59.62 \\
19 & 2 & 13.80\end{array}$ & $\begin{array}{l}+0.19 \\
+0.09\end{array}$ & $\begin{array}{l}\cdots \\
\cdots\end{array}$ & $\begin{array}{l}\cdots \\
\cdots\end{array}$ & C. & $\begin{array}{lrr}-22 & 1 & 6.6 \\
-24 & 42 & 11.5\end{array}$ & $\begin{array}{l}+2.3 \\
-0.5\end{array}$ & $\cdots$ & $\begin{array}{l}\cdots \\
\cdots\end{array}$ \\
\hline 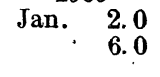 & $\begin{array}{l}\text { L. } \\
\text { L. }\end{array}$ & & $\begin{array}{l}\text { C. } \\
\text { C. }\end{array}$ & $\begin{array}{rrr}19 & 16 & 31.98 \\
19 & 45 & 4.11\end{array}$ & $\begin{array}{l}+0.15 . \\
-0.01\end{array}$ & $\cdots$ & $\cdots$ & C. & $\begin{array}{lll}-24 & 22 & 47.9 \\
-23 & 25 & 13.4\end{array}$ & $\begin{array}{l}+1.2 \\
+1.5\end{array}$ & $\cdots$ & $\cdots$ \\
\hline $\begin{array}{l}19.1 \\
20.1 \\
22.1 \\
26.1 \\
27.1\end{array}$ & $\begin{array}{l}\mathrm{P} . \\
\mathrm{L} . \\
\mathrm{P} . \\
\mathrm{L} . \\
\mathrm{P} .\end{array}$ & W. & $\begin{array}{l}\text { C. } \\
\text { I } \\
\text { C. } \\
\text { C. } \\
\text { I }\end{array}$ & $\begin{array}{lll}21 & 12 & 32.79 \\
21 & 18 & 25.86 \\
21 & 29 & 29.63 \\
21 & 47 & 46.15 \\
21 & 51 & 17.63\end{array}$ & $\begin{array}{l}+0.01 \\
-0.01 \\
+0.07 \\
-0.04 \\
+0.02\end{array}$ & $\begin{array}{l}\cdots \\
\cdots \\
\cdots \\
\cdots \\
\cdots\end{array}$ & $\begin{array}{l}\cdots \\
\cdots \\
\cdots \\
\cdots \\
\cdots\end{array}$ & $\begin{array}{l}\text { C. } \\
\text { C. } \\
\text { C. } \\
\text { C. } \\
\text { C. }\end{array}$ & $\begin{array}{lll}-17 & 31 & 28.1 \\
-16 & 55 & 48.6 \\
-15 & 42 & 58.0 \\
-13 & 18 & 22.0 \\
-12 & 44 & 22.2\end{array}$ & $\begin{array}{l}+1.1 \\
+1.7 \\
+0.8 \\
+0.5 \\
+1.3\end{array}$ & $\begin{array}{l}\cdots \\
\cdots \\
\cdots \\
\cdots \\
\cdots\end{array}$ & $\begin{array}{l}\cdots \\
\cdots \\
\cdots \\
\cdots\end{array}$ \\
\hline
\end{tabular}

$29297^{\circ}-20-33$ 


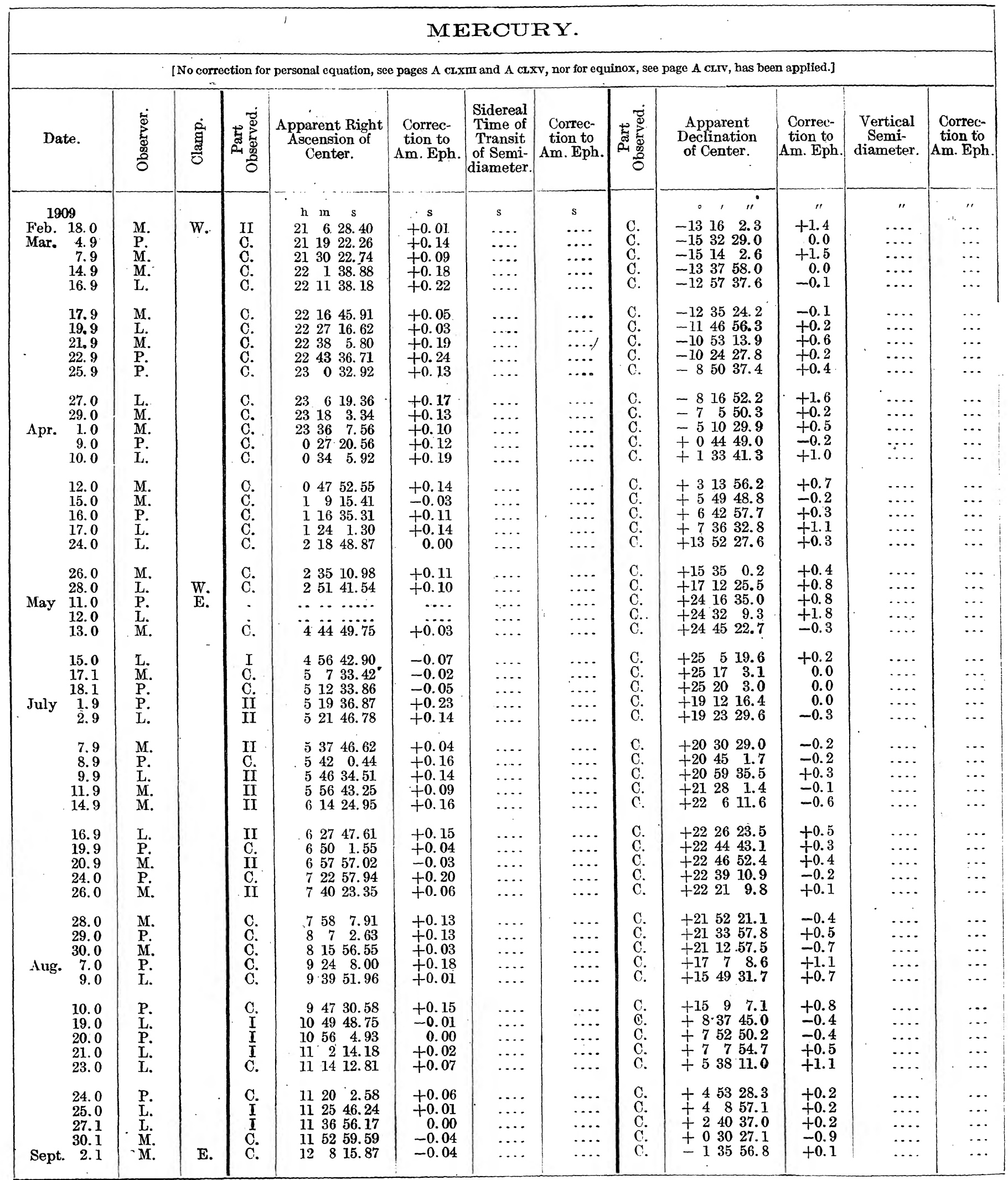




\begin{tabular}{|c|c|c|c|c|c|c|c|c|c|c|c|c|}
\hline \multicolumn{13}{|c|}{ MERCURY. } \\
\hline \multicolumn{13}{|c|}{ [No correction for personal equation, see pages $\Lambda$ CLXm and A CLXV, nor for equinox, see page A cLrv, has been applied.] } \\
\hline Date. & 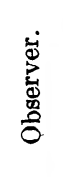 & 密 & م: & $\begin{array}{l}\text { Apparent Right } \\
\text { Ascension of } \\
\text { Center. }\end{array}$ & $\begin{array}{c}\text { Correc, } \\
\text { tion to } \\
\text { Am. Eph. }\end{array}$ & $\begin{array}{c}\text { Sidereal } \\
\text { Time of } \\
\text { Transit- } \\
\text { of Semi- } \\
\text { diameter. }\end{array}$ & $\begin{array}{c}\text { Correc- } \\
\text { tion to } \\
\text { Am. Eph. }\end{array}$ & 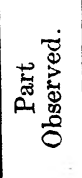 & $\begin{array}{l}\text { Apparent } \\
\text { Declination } \\
\text { of Center. }\end{array}$ & $\begin{array}{c}\text { Correc- } \\
\text { tion to } \\
\text { Am. Eph. }\end{array}$ & $\begin{array}{c}\text { Vertical } \\
\text { Semi- } \\
\text { diameter. }\end{array}$ & $\begin{array}{l}\text { Correc- } \\
\text { tion to } \\
\text { Am. Eph. }\end{array}$ \\
\hline \begin{aligned} & \multicolumn{2}{c}{1909} \\
& Sept. 7.1 \\
& 8.1 \\
& 13.1 \\
& 14.1 \\
& 17.1\end{aligned} & $\begin{array}{l}\text { P. } \\
\text { L. } \\
\text { P. } \\
\text { M. }\end{array}$ & E. & $\begin{array}{l}\text { I } \\
\text { I } \\
\text { C. } \\
\text { C. }\end{array}$ & $\begin{array}{ccc}\text { h. } & \mathrm{m} & \mathrm{s} \\
12 & 32 & 0.73 \\
12 & 36 & 29.96 \\
12 & 57 & 27.80 \\
13 & 1 & 19.76 \\
\ldots & . & \cdots\end{array}$ & $\begin{array}{c}s \\
-0.03 \\
0.00 \\
-0.01 \\
+0.10 \\
\cdots\end{array}$ & $\begin{array}{l}\mathrm{s} \\
\cdots \\
\cdots \\
\cdots \\
\cdots \\
\cdots\end{array}$ & $\begin{array}{l}\mathrm{s} \\
\cdots \\
\cdots \\
\cdots \\
\cdots \\
\cdots\end{array}$ & $\begin{array}{l}\text { C. } \\
\text { C. } \\
\text { C. } \\
\text { C. }\end{array}$ & 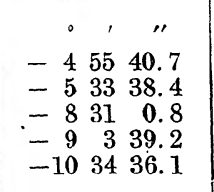 & $\begin{array}{l} \\
+1 \\
+0.3 \\
+0.1 \\
+1.3 \\
+0.1 \\
+0.1\end{array}$ & $\begin{array}{l}\prime \\
\ldots \\
\cdots \\
\cdots \\
\cdots \\
\cdots\end{array}$ & $\begin{array}{l}\prime \\
\cdots \\
\cdots \\
\cdots \\
\cdots\end{array}$ \\
\hline $\begin{array}{l}18.1 \\
23.1 \\
25.1 \\
28.1 \\
29.1\end{array}$ & $\begin{array}{l}\text { P. } \\
\text { P. } \\
\text { P. } \\
\text { L. }\end{array}$ & & $\begin{array}{l}\text { I } \\
\text { I } \\
\text { I } \\
\text { I } \\
\text { I }\end{array}$ & $\begin{array}{rrr}13 & 15 & 26.14 \\
13 & 29 & 8.32 \\
13 & 32 & 58.31 \\
13 & 36 & 25.30 \\
13 & 36 & 51.50\end{array}$ & $\begin{array}{l}-0.04 \\
+0.08 \\
-0.05 \\
-0.05 \\
-0.14\end{array}$ & $\begin{array}{l}\cdots \\
\cdots \\
\cdots \\
\cdots \\
\cdots\end{array}$ & $\begin{array}{l}\cdots \\
\cdots \\
\cdots \\
\cdots \\
\cdots\end{array}$ & $\begin{array}{l}\text { C. } \\
\text { c. } \\
\text { C. } \\
\text { C. } \\
\text { C. }\end{array}$ & $\begin{array}{rrr}-11 & 2 & 21.5 \\
-12 & 57 & 4.7 \\
-13 & 29 & 11.6 \\
-13 & 58 & 12.9 \\
-14 & 1 & 51.5\end{array}$ & $\begin{array}{l}-0.8 \\
-1.5 \\
-0.7 \\
-1.1 \\
+0.7\end{array}$ & $\begin{array}{l}\cdots \\
\cdots \\
\cdots \\
\cdots \\
\cdots\end{array}$ & $\begin{array}{l}\cdots \\
\cdots \\
\cdots \\
\cdots\end{array}$ \\
\hline 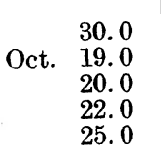 & $\begin{array}{l}\text { P. } \\
\text { P. } \\
\text { M. } \\
\text { M. }\end{array}$ & & $\begin{array}{l}\text { I } \\
\text { C. } \\
\text { II } \\
\text { II }\end{array}$ & $\begin{array}{llll}13 & 36 & 54.04 \\
12 & 46 & 46.76 \\
12 & 46 & 12.49 \\
\dddot{12} & 0 & 32 & 42.90\end{array}$ & $\begin{array}{l}-0.11 \\
+0.34 \\
+0.06 \\
+0.10\end{array}$ & $\begin{array}{l}\cdots \\
\cdots \\
\cdots \\
\cdots \\
\cdots\end{array}$ & $\begin{array}{l}\cdots \\
\cdots \\
\cdots \\
\cdots \\
\cdots\end{array}$ & $\begin{array}{l}\text { C. } \\
\text { C. } \\
\text { C. } \\
\text { c. }\end{array}$ & $\begin{array}{rrr}-14 & 2 & 9.1 \\
-4 & 21 & 25.5 \\
-4 & 0 & 14.0 \\
-3 & 35 & 18.0 \\
-3 & 39 & 38.4\end{array}$ & $\begin{array}{l}-0.8 \\
+0.5 \\
+1.0 \\
-0.2 \\
-0.5\end{array}$ & $\begin{array}{l}\cdots \\
\cdots \\
\cdots \\
\cdots\end{array}$ & $\begin{array}{l}\cdots \\
\cdots \\
\cdots \\
\cdots\end{array}$ \\
\hline $\begin{array}{r}26.0 \\
27.0 \\
29.0 \\
\text { Nov. } 2.0 \\
3.0\end{array}$ & $\begin{array}{l}\text { P. } \\
\text { L. } \\
\text { P. } \\
\text { L. }\end{array}$ & & $\begin{array}{l}\text { II } \\
\text { II } \\
\text { II } \\
\text { II } \\
\text { II }\end{array}$ & $\begin{array}{rrr}12 & 55 & 39.52 \\
12 & 59 & 2.08 \\
13 & 6 & 56.02 \\
13 & 26 & 4.69 \\
13 & 31 & 21.54\end{array}$ & $\begin{array}{l}+0.36 \\
+0.10 \\
+0.35 \\
+0.29 \\
+0.19\end{array}$ & $\begin{array}{l}\cdots \\
\cdots \\
\cdots \\
\cdots \\
\cdots\end{array}$ & $\begin{array}{l}\cdots \\
\cdots \\
\cdots \\
\cdots \\
\cdots\end{array}$ & $\begin{array}{l}\text { C. } \\
\text { C. } \\
\text { c. } \\
\text { c. }\end{array}$ & $\begin{array}{rrr} & 350 & 58.5 \\
- & 6 & 34.4 \\
- & 48 & 46.6 \\
-6 & 45 & 9.4 \\
- & 718 & 43.0\end{array}$ & $\begin{array}{l}+0.3 \\
+0.9 \\
+0.1 \\
-0.2 \\
+0.9\end{array}$ & $\begin{array}{l}\cdots \\
\cdots \\
\cdots \\
\cdots \\
\cdots\end{array}$ & $\begin{array}{l}\cdots \\
\cdots \\
\cdots \\
\cdots \\
\cdots\end{array}$ \\
\hline $\begin{array}{r}4.0 \\
5.0 \\
11.0 \\
12.0 \\
13.0\end{array}$ & $\begin{array}{l}\text { M. } \\
\text { L. } \\
\text { M. } \\
\text { L. } \\
\text { M. }\end{array}$ & & $\begin{array}{l}\mathrm{C} . \\
\text { UI } \\
\mathrm{C} . \\
\text { II } \\
\text { C. }\end{array}$ & $\begin{array}{lll}13 & 36 & 47.13 \\
13 & 42 & 20.19 \\
14 & 17 & 24.20 \\
14 & 23 & 27.07 \\
14 & 29 & 32.28\end{array}$ & $\begin{array}{l}+0.18 \\
+0.21 \\
+0.12 \\
+0.16 \\
+0.05\end{array}$ & $\begin{array}{l}\cdots \\
\cdots \\
\cdots \\
\cdots \\
\cdots\end{array}$ & $\begin{array}{l}\cdots \\
\cdots \\
\cdots \\
\cdots \\
\cdots\end{array}$ & $\begin{array}{l}\text { c. } \\
\text { c. } \\
\text { c. } \\
\text { c. } \\
\text { c. }\end{array}$ & $\begin{array}{rrr}-7 & 53 & 28.8 \\
= & 29 & 6.4 \\
-12 & 11 & 3.8 \\
-12.47 & 59.1 \\
-13 & 24 & 34.6\end{array}$ & $\begin{array}{r}0.0 \\
+1.9 \\
+0.8 \\
+0.2 \\
0.0\end{array}$ & $\begin{array}{l}\cdots \\
\cdots \\
\cdots \\
\cdots \\
\cdots\end{array}$ & $\begin{array}{l}\cdots \\
\cdots \\
\cdots \\
\cdots \\
\cdots\end{array}$ \\
\hline 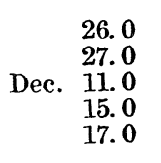 & $\begin{array}{l}\text { P. } \\
\text { L. } \\
\text { L. } \\
\text { L. } \\
\text { L. }\end{array}$ & & $\begin{array}{l}\text { c. } \\
\text { C. } \\
\text { c. } \\
\text { C. }\end{array}$ & $\begin{array}{rrrr}15 & 51 & 33.44 \\
15 & 58 & 4.12 \\
18 & \because & 77.86 \\
18 & 0 & 17.86 \\
18 & 14 & 20.29\end{array}$ & $\begin{array}{l}+0.26 \\
+0.19 \\
+0.15 \\
+0.19\end{array}$ & $\begin{array}{l}\cdots \\
\cdots \\
\cdots \\
\cdots \\
\cdots\end{array}$ & $\begin{array}{l}\cdots \\
\cdots \\
\cdots \\
\cdots \\
\cdots\end{array}$ & $\begin{array}{l}\text { C. } \\
\text { C. } \\
\text { C. } \\
\text { C. }\end{array}$ & $\begin{array}{rrr}-20 & 22 & 8.6 \\
-20 & 48 & 15.8 \\
-24 & 56 & 18.7 \\
-25 & 21 & 17.9 \\
-25 & 25 & 12.5\end{array}$ & $\begin{array}{l}+0.6 \\
+1.6 \\
+2.0 \\
+1.3 \\
+2.4\end{array}$ & $\begin{array}{l}\cdots \\
\cdots \\
\cdots \\
\cdots \\
\cdots\end{array}$ & $\begin{array}{l}\cdots \\
\cdots \\
\cdots \\
\cdots\end{array}$ \\
\hline $\begin{array}{r}21.0 \\
22.0 \\
23.0 \\
24.0 \\
1910\end{array}$ & $\begin{array}{l}\text { P. } \\
\text { L. } \\
\text { M. }\end{array}$ & & $\begin{array}{l}\text { C. } \\
\text { C. } \\
\text { C. } \\
\text { C. }\end{array}$ & $\begin{array}{rrr}18 & 42 & 27.88 \\
18 & 49 & 28.80 \\
18 & 56 & 28.53 \\
19 & 3 & 26.84\end{array}$ & $\begin{array}{l}+0.20 \\
+0.23 \\
+0.13 \\
+0.09\end{array}$ & $\begin{array}{l} \\
\cdots \\
\cdots \\
\cdots \\
\cdots\end{array}$ & $\begin{array}{l}\cdots \\
\cdots \\
\cdots \\
\cdots\end{array}$ & $\begin{array}{l}\text { C. } \\
\text { C. } \\
\text { C. } \\
\text { C. }\end{array}$ & $\begin{array}{rrr}-25 & 15 & 13.5 \\
-25 & 8 & 53.2 \\
-25 & 1 & 4.6 \\
-24 & 51 & 35.9\end{array}$ & $\begin{array}{l}+0.3 \\
+1.6 \\
-1.6 \\
+2.2\end{array}$ & $\begin{array}{l}\cdots \\
\cdots \\
\cdots \\
\cdots\end{array}$ & $\begin{array}{l}\cdots \\
\cdots \\
\cdots \\
\cdots\end{array}$ \\
\hline $\begin{array}{lr}\text { Jan. } & 7.1 \\
8.1 \\
10.1 \\
15.1 \\
\text { Feb. } \quad 2.0\end{array}$ & $\begin{array}{l}\text { P. } \\
\mathrm{L} . \\
\mathrm{M} . \\
\mathrm{L} . \\
\mathrm{P} .\end{array}$ & & $\begin{array}{l}\text { C. } \\
\text { C. } \\
\text { I } \\
\text { I } \\
\text { C. }\end{array}$ & $\begin{array}{lll}20 & 31 & 50.22 \\
20 & 36 & 41.50 \\
20 & 45 & 15.77 \\
20 & 57 & 41.42 \\
19 & 57 & 20.34\end{array}$ & $\begin{array}{l}+0.01 \\
-0.03 \\
+0.01 \\
-0.15 \\
+0.32\end{array}$ & $\begin{array}{l}\cdots \\
\cdots \\
\cdots \\
\cdots \\
\cdots\end{array}$ & $\begin{array}{l}\cdots \\
\cdots \\
\cdots \\
\cdots \\
\cdots\end{array}$ & $\begin{array}{l}\text { C. } \\
\text { C. } \\
\text { C. } \\
\text { C. } \\
\text { C. }\end{array}$ & $\begin{array}{rrr}-20 & 9 & 8.3 \\
-19 & 41 & 3.1 \\
-18 & 44 & 36.2 \\
-16 & 36 & 1.5 \\
-17 & 18 & 58.2\end{array}$ & $\begin{array}{l}+0.1 \\
+2.7 \\
+1.0 \\
+1.3 \\
+2.6\end{array}$ & $\begin{array}{l}\cdots \\
\cdots \\
\cdots \\
\cdots \\
\cdots\end{array}$ & $\begin{array}{l}\cdots \\
\cdots \\
\cdots \\
\cdots\end{array}$ \\
\hline $\begin{array}{r}5.0 \\
18.9 \\
24.9 \\
\text { Mar. } \quad 3.9 \\
6.9\end{array}$ & $\begin{array}{l}\text { L. } \\
\mathrm{L} . \\
\mathrm{P} . \\
\mathrm{P} . \\
\mathrm{M} .\end{array}$ & & $\begin{array}{l}\text { C. } \\
\text { C. } \\
\text { C. } \\
\text { C. } \\
\text { C. }\end{array}$ & $\begin{array}{rrr}19 & 52 & 34.68 \\
20 & 23 & 24.94 \\
20 & 51 & 28.18 \\
21 & 29 & 7.75 \\
21 & 46 & 19.74\end{array}$ & $\begin{array}{l}+0.18 \\
+0.23 \\
+0.22 \\
+0.18 \\
+0.09\end{array}$ & $\begin{array}{l}\cdots \\
\cdots \\
\cdots \\
\cdots \\
\cdots\end{array}$ & $\begin{array}{l}\cdots \\
\cdots \\
\cdots \\
\cdots \\
\cdots\end{array}$ & $\begin{array}{l}\text { C. } \\
\text { C. } \\
\text { C. } \\
\text { C. } \\
\text { C. }\end{array}$ & $\begin{array}{rrr}-18 & 0 & 5.3 \\
-19 & 8 & 32.8 \\
-18 & 22 & 39.6 \\
-16 & 29 & 21.3 \\
-15 & 21 & 13.6\end{array}$ & $\begin{array}{l}+1.2 \\
+1.6 \\
+0.8 \\
+0.3 \\
-0.4\end{array}$ & $\begin{array}{l}\cdots \\
\cdots \\
\cdots \\
\cdots \\
\cdots\end{array}$ & $\begin{array}{l}\cdots \\
\cdots \\
\cdots \\
\cdots \\
\cdots\end{array}$ \\
\hline $\begin{array}{r}7.9 \\
16.0 \\
21.0 \\
23.0 \\
\text { Apr. } \quad 2.0\end{array}$ & $\begin{array}{l}\mathrm{P} . \\
\mathrm{M} . \\
\mathrm{M} . \\
\mathrm{L} .\end{array}$ & & $\begin{array}{l}\text { C. } \\
\text { C. } \\
\text { C. } \\
\text { C. } \\
\text { C. }\end{array}$ & $\begin{array}{rrr}21.52 & 10.57 \\
22 & 40 & 37.04 \\
23 & 12 & 20.34 \\
23 & 25 & 21.82 \\
0 & 34 & 1.10\end{array}$ & $\begin{array}{l}+0.25 \\
+0.13 \\
+0.09 \\
+0.10 \\
+0.03\end{array}$ & $\begin{array}{l} \\
\cdots \\
\cdots \\
\cdots \\
\cdots\end{array}$ & $\begin{array}{l}\cdots \\
\cdots \\
\cdots \\
\cdots \\
\cdots\end{array}$ & $\begin{array}{l}\text { C. } \\
\text { c. } \\
\text { C. } \\
\text { C. }\end{array}$ & $\begin{array}{rrr}-14 & 55 & 54.4 \\
-10 & 47 & 51.7 \\
-7 & 32 & 36.1 \\
-6 & 6 & 9.1 \\
+2 & 10 & 20.4\end{array}$ & $\begin{array}{l}+1.1 \\
+0.3 \\
+1.1 \\
+2.1 \\
+0.8\end{array}$ & $\begin{array}{l}\cdots \\
\cdots \\
\cdots \\
\cdots \\
\cdots\end{array}$ & $\begin{array}{l}\cdots \\
\cdots \\
\cdots \\
\cdots\end{array}$ \\
\hline $\begin{array}{r}8.0 \\
9.0 \\
13.0 \\
14.0 \\
15.0\end{array}$ & $\begin{array}{l}\text { P. } \\
\text { L. } \\
\text { L. } \\
\text { M. } \\
\text { P. }\end{array}$ & E. & $\begin{array}{c}\text { I } \\
\text { C. } \\
\text { C. } \\
\text { I } \\
\text { I }\end{array}$ & $\begin{array}{rrr}1 & 18 & 26.26 \\
1 & 26 & 2.93 \\
1 & 56 & 46.10 \\
2 & 4 & 26.80 \\
2 & 12 & 5.47\end{array}$ & $\begin{array}{r}+0.04 \\
+0.13 \\
+0.12 \\
+0.04 \\
0.00\end{array}$ & $\begin{array}{l}\cdots \\
\cdots \\
\cdots \\
\cdots \\
\cdots\end{array}$ & $\begin{array}{l}\cdots \\
\cdots \\
\cdots \\
\cdots \\
\cdots\end{array}$ & $\begin{array}{l}\text { c. } \\
\text { C. } \\
\text { c. } \\
\text { C. }\end{array}$ & $\begin{array}{rrr}+744 & 38.2 \\
+841 & 0.7 \\
+12 & 2156.7 \\
+13 & 1456.4 \\
+146 & 60.6\end{array}$ & $\begin{array}{l}+0.3 \\
+0.7 \\
+1.6 \\
-1.0 \\
-0.2\end{array}$ & $\begin{array}{l}\ldots \\
\cdots \\
\cdots \\
\cdots \\
\cdots\end{array}$ & $\begin{array}{l}\cdots \\
\cdots \\
\cdots \\
\cdots\end{array}$ \\
\hline
\end{tabular}




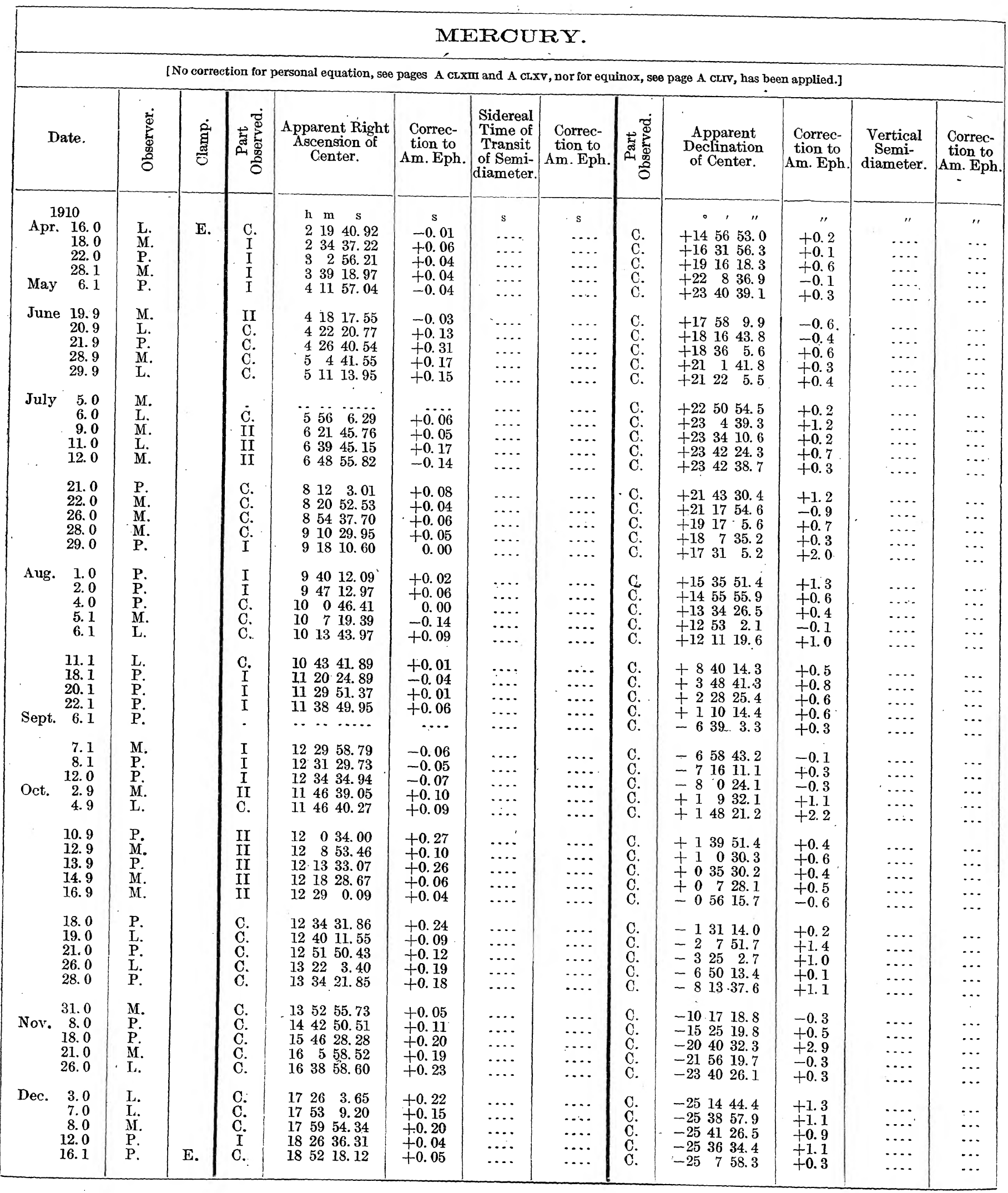




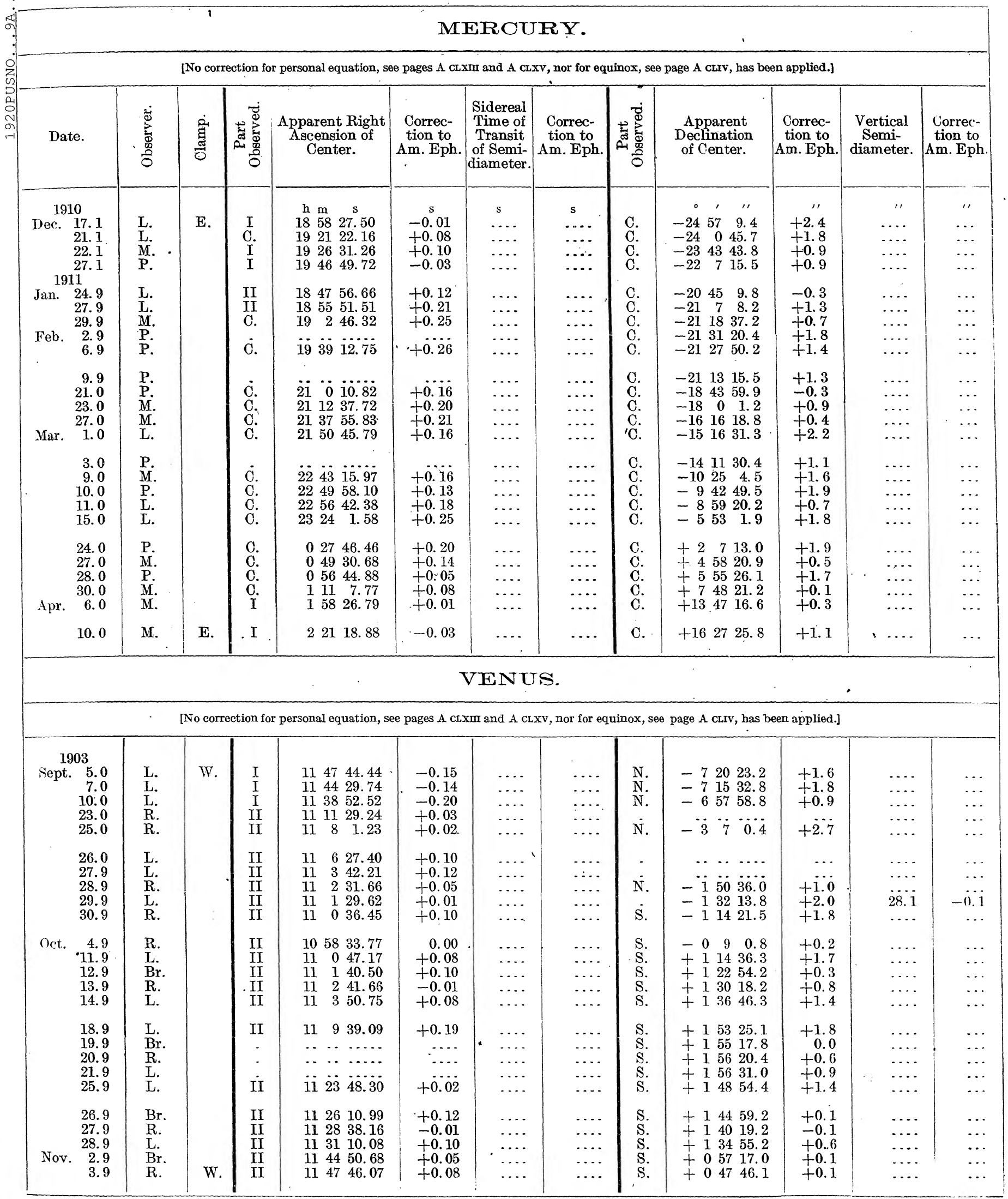




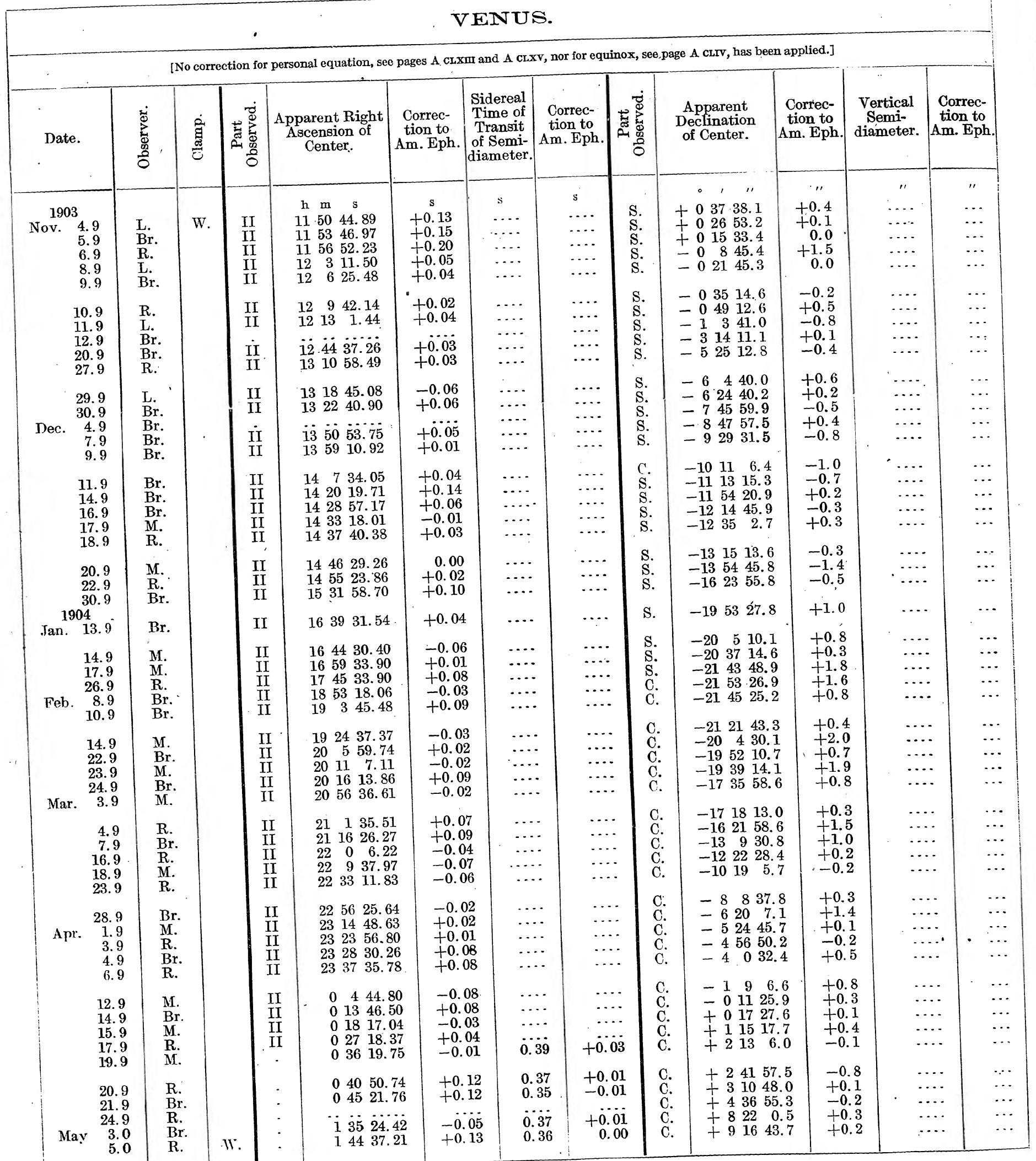




\begin{tabular}{|c|c|c|c|c|c|c|c|c|c|c|c|c|}
\hline \multicolumn{13}{|c|}{ VENUS. } \\
\hline \multicolumn{13}{|c|}{ [No correction for personal équation, see pages A cLxar and A cLxv, nor for equinox, see page A cLIv, has becn applied.] } \\
\hline Date. & 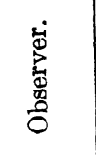 & 灾 & 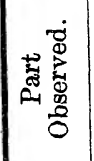 & $\begin{array}{l}\text { Apparent Right } \\
\text { Ascension of } \\
\text { Cénter. }\end{array}$ & $\begin{array}{c}\text { Correc- } \\
\text { tion to } \\
\text { Am. Eph. }\end{array}$ & $\begin{array}{c}\text { Sidereal } \\
\text { Time of } \\
\text { Transit } \\
\text { of Semi- } \\
\text { diameter. }\end{array}$ & $\begin{array}{c}\text { Correc- } \\
\text { tion to } \\
\text { Am. Eph. }\end{array}$ & 蛋 & $\begin{array}{l}\text { Apparent } \\
\text { Declination } \\
\text { of Center. }\end{array}$ & $\begin{array}{c}\text { Correc- } \\
\text { tion to } \\
\text { Am. Eph. }\end{array}$ & $\begin{array}{c}\text { Vertical } \\
\text { Semi- } \\
\text { diameter. }\end{array}$ & $\begin{array}{l}\text { Correc- } \\
\text { tion to } \\
\text { Am. Eph. }\end{array}$ \\
\hline 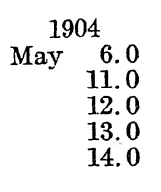 & $\begin{array}{l}\text { Br. } \\
\text { M. } \\
\text { R. } \\
\text { Br. } \\
\text { M. }\end{array}$ & W. & $\because$ & $\begin{array}{lcc}\mathrm{h} & \mathrm{m} & \mathrm{s} \\
1 & 49 & 14.53 \\
2 & 12 & 32.75 \\
2 & 17 & 14.86 \\
2 & 21 & 57.93 \\
2 & 26 & 41.95\end{array}$ & $\begin{array}{c}\mathrm{s} \\
+0.12 \\
+0.07 \\
+0.02 \\
+0.03 \\
+0.06\end{array}$ & $\begin{array}{l}\mathrm{s} \\
0.34 \\
0.37 \\
0.36 \\
0.33 \\
0.32\end{array}$ & $\begin{array}{c}\mathrm{s} \\
-0.01 \\
+0.02 \\
+0.01 \\
-0.02 \\
-0.03\end{array}$ & $\begin{array}{l}\text { C. } \\
\text { C. } \\
\text { C. } \\
\text { C. }\end{array}$ & $\begin{array}{rcc}\circ & , 1 \\
+9 & 43 & 46.9 \\
+11 & 55 & 40.3 \\
+12 & 21 & 17.1 \\
+12 & 46 & 35.5 \\
+13 & 11 & 36.2\end{array}$ & $\begin{array}{r} \\
-0.5 \\
+0.3 \\
+0.9 \\
+0.4 \\
.0 .0\end{array}$ & $\begin{array}{l}. \\
\ldots \\
\cdots \\
\cdots \\
\cdots \\
\cdots\end{array}$ & $\begin{array}{l}\text { " } \\
\cdots \\
\cdots \\
\cdots \\
\cdots \\
\cdots\end{array}$ \\
\hline $\begin{array}{r}16.0 \\
25.0 \\
26.0 \\
28.0 \\
\text { June } 6.0\end{array}$ & $\begin{array}{l}\mathrm{R} . \\
\mathrm{R} . \\
\mathrm{Br} . \\
\mathrm{Br} . \\
\mathrm{Br} .\end{array}$ & & $:$ & $\begin{array}{lll}2 & 36 & 12.84 \\
3 & 19 & 52.34 \\
3 & 24 & 48.83 \\
3 & 34 & 45.24 \\
4 & 20 & 24.03\end{array}$ & $\begin{array}{l}+0.12 \\
+0.02 \\
+0.03 \\
+0.1 .0 \\
+0.03\end{array}$ & $\begin{array}{l}0.33 \\
0.37 \\
0.34 \\
0.35 \\
0.34\end{array}$ & $\begin{array}{r}-0.02 \\
+0.02 \\
-0.01 \\
0.00 \\
-0.02\end{array}$ & $\begin{array}{l}\text { C. } \\
\text { C. } \\
\text { C. } \\
\text { c. }\end{array}$ & $\begin{array}{rrr}+14 & 0 & 41.4 \\
+17 & 23 & 37.6 \\
+17 & 44 & 7.6 \\
+18 & 23 & 43.6 \\
+20 & 57 & 1.6\end{array}$ & $\begin{array}{r}-0.4 \\
0.0 \\
+0.6 \\
+0.2 \\
0.0\end{array}$ & $\begin{array}{l}\cdots \\
\cdots \\
\cdots \\
\cdots\end{array}$ & $\begin{array}{l}\ldots \\
\cdots \\
\cdots \\
\cdots \\
\cdots\end{array}$ \\
\hline $\begin{array}{r}8.0 \\
9.0 \\
11.0 \\
13.0 \\
14.0\end{array}$ & $\begin{array}{l}\text { Br. } \\
\text { R. } \\
\text { M. } \\
\text { R. } \\
\text { Br. }\end{array}$ & & $\dot{-}$ & $\begin{array}{rrr}4 & 30 & 44.56 \\
4 & 35 & 56.25 \\
4 & 46 & 22.44 \\
4 & 56 & 52.26 \\
5 & 2 & 8.50\end{array}$ & $\begin{array}{l}+0.12 \\
+0.10 \\
+0.03 \\
+0.02 \\
+0.12\end{array}$ & $\begin{array}{l}0.36 \\
0.40 \\
0.39 \\
0.35 \\
0.32\end{array}$ & $\begin{array}{r}0.00 \\
+0.04 \\
+0.03 \\
-0.01 \\
-0.04\end{array}$ & $\begin{array}{l}\text { C. } \\
\text { C. } \\
\text { C. } \\
\text { C. } \\
\text { C. }\end{array}$ & 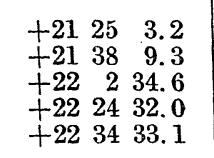 & $\begin{array}{l}+0.7 \\
-0.6 \\
-0.8 \\
+0.2 \\
+0.1\end{array}$ & $\begin{array}{l}\cdots \\
\cdots \\
\cdots \\
\cdots \\
\cdots\end{array}$ & $\begin{array}{l}\cdots \\
\cdots \\
\cdots \\
\cdots\end{array}$ \\
\hline $\begin{array}{ll} & 15.0 \\
\text { July } & 19.0 \\
26.0 \\
27.0 \\
29.0\end{array}$ & $\begin{array}{l}\text { M. } \\
\text { Br. } \\
\text { Br. } \\
\text { T. } \\
\text { Br. }\end{array}$ & & $\begin{array}{l}\dot{C} . \\
\text { C. } \\
\text { C. }\end{array}$ & $\begin{array}{rrr}5 & 7 & 25.40 \\
8 & 8 & 30.02 \\
8 & 44 & 25.69 \\
8 & 49 & 29.21 \\
8 & 59 & 33.00\end{array}$ & $\begin{array}{l}+0.10 \\
+0.07 \\
+0.04 \\
-0.01 \\
+0.04\end{array}$ & $\begin{array}{l}0.33 \\
\cdots \\
\cdots \\
\ldots .30 \\
.0 .30\end{array}$ & \begin{tabular}{r}
-0.03 \\
$\ldots$ \\
$\cdots$ \\
\hdashline 0 \\
-0.05
\end{tabular} & $\begin{array}{l}\text { c. } \\
\text { c. } \\
\text { C. } \\
\text { C. } \\
\text { C. }\end{array}$ & $\begin{array}{rrr}+22 & 43 & 55.3 \\
+21 & 14 & 53.8 \\
+19 & 21 & 53.3 \\
+19 & 3 & 28.3 \\
+18 & 25 & 2.8\end{array}$ & $\begin{array}{l}-0.2 \\
+0.1 \\
-0.2 \\
-0.3 \\
-0.2\end{array}$ & $\begin{array}{l}\cdots \\
\cdots \\
\cdots \\
\cdots \\
\cdots\end{array}$ & $\begin{array}{l}\cdots \\
\cdots \\
\cdots \\
\cdots\end{array}$ \\
\hline 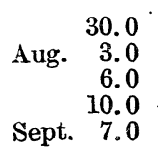 & $\begin{array}{l}\mathrm{M} . \\
\mathrm{T} . \\
\mathrm{T} . \\
\mathrm{T} . \\
\mathrm{M} .\end{array}$ & $\begin{array}{l}\text { W. } \\
\text { E. }\end{array}$ & $\begin{array}{l}\text { C. } \\
\text { C.: } \\
\text { C. } \\
\therefore\end{array}$ & $\begin{array}{rrr}9 & 4 & 32.98 \\
9 & 24 & 22.12 \\
9 & 39 & 2.01 \\
9 & 58 & 19.77 \\
12 & 7 & 12.20\end{array}$ & $\begin{array}{l}-0.12 \\
-0.06 \\
-0.02 \\
+0.03 \\
-0.03\end{array}$ & $\begin{array}{l}\cdots \\
\cdots \\
\cdots \\
0.31\end{array}$ & $\begin{array}{c}\ldots . \\
\cdots \\
-0.0 \\
-0.04\end{array}$ & $\begin{array}{l}\text { C. } \\
\text { C. } \\
\text { C. } \\
\text { C. } \\
\text { C. }\end{array}$ & $\begin{array}{rrr}+185 & 5.8 \\
+16 & 40 & 8.7 \\
+1531 & 36.5 \\
+13 & 54 & 21.0 \\
+0 & 29 & 19.7\end{array}$ & $\begin{array}{r}-0.6 \\
+0.1 \\
+0.2 \\
0.0 \\
+0.8\end{array}$ & $\begin{array}{l}\cdots \\
\cdots \\
\cdots \\
\cdots \\
\cdots\end{array}$ & $\begin{array}{l}\cdots \\
\cdots \\
\cdots \\
\cdots \\
\cdots\end{array}$ \\
\hline $\begin{array}{r}8.0 \\
12.0 \\
15.0 \\
16.0 \\
17.0\end{array}$ & $\begin{array}{l}\mathrm{T}: \\
\mathrm{M} . \\
\mathrm{T} . \\
\mathrm{M} .\end{array}$ & & $\begin{array}{l}\text { c. } \\
\dot{\text { c. }} \\
\dot{-}\end{array}$ & $\begin{array}{lll}12 & 11 & 41.94 \\
12 & 29 & 40.92 \\
12 & 43 & 11.36 \\
12 & 47 & 41.93 \\
12 & 52 & 12.76\end{array}$ & $\begin{array}{r}-0.03 \\
-0.04 \\
0.00 \\
+0.01 \\
0.00\end{array}$ & $\begin{array}{l}0.33 \\
0.34 \\
0.41\end{array}$ & $\begin{array}{l}-0.03 \\
-0.02 \\
+0.05\end{array}$ & $\begin{array}{l}\text { C. } \\
\text { C: } \\
\dot{c} . \\
\text { c. }\end{array}$ & $\begin{array}{rrr}-0 & 1 & 32.4 \\
-2 & 5 & 1.4 \\
-\because & \because & \because \\
-4 & 8 & 6.0 \\
-4 & 38 & 44.8\end{array}$ & $\begin{array}{l}-0.7 \\
-0.5 \\
+0.1 \\
-1.8\end{array}$ & $\begin{array}{l}\cdots \\
\cdots \\
\cdots \\
\cdots \\
\cdots\end{array}$ & $\begin{array}{l}\cdots \\
\cdots \\
\cdots \\
\cdots\end{array}$ \\
\hline $\begin{array}{l}19.0 \\
21.0 \\
22.0 \\
23.0 \\
26.1\end{array}$ & $\begin{array}{l}\text { M. } \\
\text { M. } \\
\text { T. } \\
\text { M. } \\
\text { M. }\end{array}$ & & $\dot{\dot{I}}$ & $\begin{array}{lll}13 & 1 & 15.51 \\
13 & 10 & 19.83 \\
13 & 14 & 52.84 \\
13 & 19 & 26.45 \\
13 & 33 & 10.51\end{array}$ & $\begin{array}{l}+0.01 \\
-0.12 \\
-0.06 \\
+0.06 \\
+0.01\end{array}$ & $\begin{array}{l}0.34 \\
0.35 \\
0.46 \\
\cdots . . \\
\cdots\end{array}$ & $\begin{array}{c}-0.02 \\
-0.02 \\
+0.09 \\
\ldots . \\
\cdots\end{array}$ & $\begin{array}{l}\text { c. } \\
\text { c. } \\
\text { c. } \\
\text { c. }\end{array}$ & 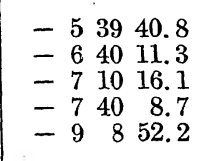 & $\begin{array}{l}-0.4 \\
-0.1 \\
-1.2 \\
+0.9 \\
+0.2\end{array}$ & $\begin{array}{l}\cdots \\
\cdots \\
\cdots \\
\cdots \\
\cdots\end{array}$ & $\begin{array}{l}\cdots \\
\cdots \\
\cdots \\
\cdots\end{array}$ \\
\hline $\begin{array}{cr} & 28.1 \\
& 30.1 \\
\text { Oct. } \quad 1.1 \\
3.1 \\
4.1\end{array}$ & $\begin{array}{l}\mathrm{M} . \\
\mathrm{M} . \\
\mathrm{M} . \\
\mathrm{Br} .\end{array}$ & & $\begin{array}{l}\mathrm{I} \\
\mathrm{I} \\
\mathrm{I} \\
\mathrm{I} \\
.\end{array}$ & $\begin{array}{rrr}13 & 42 & 23.44 \\
13 & 51 & 39.28 \\
13 & 56 & 18.56 \\
14 & 5 & 39.95 \\
14 & 10 & 22.06\end{array}$ & $\begin{array}{l}+0.09 \\
-0.31 \\
-0.11 \\
+0.01 \\
+0.06\end{array}$ & $\begin{array}{l}\ldots . . \\
\cdots \\
\cdots . . \\
0.38\end{array}$ & $\begin{array}{c}\ldots . \\
\cdots \ldots \\
\cdots \\
0.00\end{array}$ & $\begin{array}{l}\text { c. } \\
\text { C. } \\
\text { C. } \\
\text { C. }\end{array}$ & $\begin{array}{rrr}-10 & 7 & 2.2 \\
-11 & 4 & 19.2 \\
-11 & 32 & 36.8 \\
-12 & 28 & 21.4 \\
-12 & 55 & 47.6\end{array}$ & $\begin{array}{r}+0.6 \\
+0.5 \\
-0.6 \\
0.0 \\
+0.8\end{array}$ & $\begin{array}{l}\ldots \\
\cdots \\
\cdots \\
\cdots \\
\cdots \\
\cdots\end{array}$ & $\begin{array}{l}\cdots \\
\cdots \\
\cdots \\
\cdots\end{array}$ \\
\hline $\begin{array}{r}5.1 \\
7.1 \\
8.1 \\
10.1 \\
14.1\end{array}$ & $\begin{array}{l}\text { M. } \\
\text { Br. } \\
\text { M. } \\
\text { M. } \\
\text { Br. }\end{array}$ & & $\begin{array}{l}\text { I } \\
\text { I } \\
\text { I } \\
.\end{array}$ & $\begin{array}{rrr}14 & 15 & 4 . \dot{99} \\
14 & 24 & 34.22 \\
14 & 29 & 20.38 \\
14 & 38 & 56.19 \\
14 & 58 & 21.32\end{array}$ & $\begin{array}{l}-0.08 \\
-0.07 \\
-0.11 \\
+0.01 \\
+0.07\end{array}$ & $\begin{array}{l}0.4 i \\
\ldots . \\
\ddot{0.4 i}\end{array}$ & $\begin{array}{r}+\ddot{0.03} \\
\ldots \ldots . \\
+0.01\end{array}$ & $\begin{array}{l}\text { C. } \\
\text { C. } \\
\text { C. } \\
\text { C. }\end{array}$ & $\begin{array}{lll}-13 & 22 & 56.6 \\
-14 & 16 & 17.4 \\
-14 & 42 & 28.7 \\
-15 & 33 & 46.0 \\
-17 & 11 & 39.6\end{array}$ & $\begin{array}{l}+0.9 \\
+0.8 \\
-0.2 \\
-1.0 \\
-0.9\end{array}$ & $\begin{array}{l}\cdots \\
\cdots \\
\cdots \\
\cdots \\
\cdots\end{array}$ & $\begin{array}{l}\cdots \\
\cdots \\
\cdots \\
\cdots\end{array}$ \\
\hline $\begin{array}{l}15.1 \\
17.1 \\
18.1 \\
19.1 \\
21.1\end{array}$ & $\begin{array}{l}\mathrm{Y} . \\
\mathrm{M} . \\
\mathrm{Br} . \\
\mathrm{Mr} .\end{array}$ & & $\begin{array}{l}\mathrm{I} \\
\mathrm{I} \\
\mathrm{I} \\
-\end{array}$ & $\begin{array}{rrr}15 & 3 & 15.65 \\
15 & 13 & 7.46 \\
15 & 18 & 5.26 \\
15 & 23 & 4.35 \\
15 & 33 & 6.02\end{array}$ & $\begin{array}{r}+0.19 \\
0.00 \\
-0.02 \\
+0.03 \\
-0.05\end{array}$ & $\begin{array}{l}\cdots . . \\
\dddot{0.41} \\
\dddot{4} . \\
0.39\end{array}$ & $\begin{array}{r}\cdots . . \\
0.00 \\
-0.03\end{array}$ & $\begin{array}{l}\dot{c} . \\
\text { c. } \\
\text { c. } \\
\text { c. }\end{array}$ & $\begin{array}{rrr}-18 & 20 & 34.3 \\
-18 & 42 & 36.1 \\
-19 & 4 & 10.1 \\
-19 & 45 & 47.6\end{array}$ & $\begin{array}{l}-0.3 \\
+0.8 \\
+0.7 \\
+1.0\end{array}$ & $\begin{array}{l}\cdots \\
\cdots \\
\cdots \\
\cdots \\
\cdots\end{array}$ & $\begin{array}{l}\cdots \\
\cdots \\
\cdots \\
\cdots\end{array}$ \\
\hline $\begin{array}{l}25.1 \\
27.1 \\
28.1 \\
29.1 \\
31.1\end{array}$ & $\begin{array}{l}\text { Br. } \\
\text { Y. } \\
\text { Br. } \\
\text { Y. } \\
\text { M. }\end{array}$ & E. & $\dot{:}$ & $\begin{array}{rrr}15 & 53 & 24.05 \\
16 & 3 & 40.14 \\
16 & 8 & 49.91 \\
16 & 14 & 0.86 \\
16 & 24 & 25.85\end{array}$ & $\begin{array}{l}+0.01 \\
+0.01 \\
-0.01 \\
+0.02 \\
+0.09\end{array}$ & $\begin{array}{l}0.44 \\
0.39 \\
0.44 \\
0.35 \\
0.33\end{array}$ & $\begin{array}{r}+0.01 \\
-0.04 \\
0.00 \\
-0.09 \\
-0.12\end{array}$ & $\begin{array}{l}\text { c. } \\
\text { c. } \\
\text { c. } \\
\text { c. } \\
\text { C. }\end{array}$ & $\begin{array}{lrr}-21 & 2 & 40.6 \\
-21 & 37 & 48.1 \\
-21 & 54 & 23.3 \\
-22 & 10 & 27.4 \\
-22 & 40 & 41.7\end{array}$ & $\begin{array}{l}+1.4 \\
-2.2 \\
+1.6 \\
+0.2 \\
+0.1\end{array}$ & $\begin{array}{l}\cdots \\
\cdots \\
\cdots \\
\cdots \\
\cdots\end{array}$ & $\begin{array}{l}\cdots \\
\cdots \\
\cdots \\
\cdots\end{array}$ \\
\hline
\end{tabular}




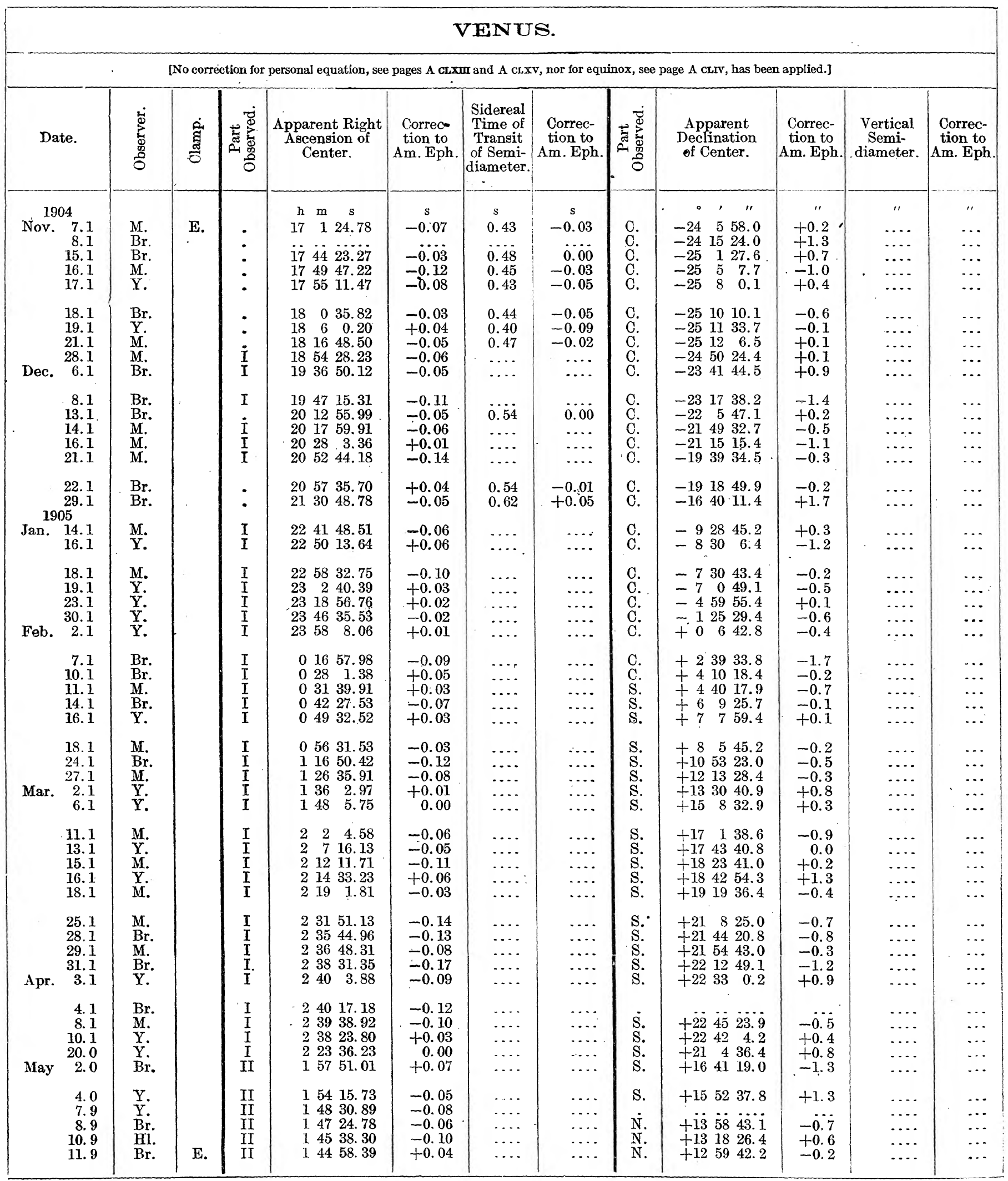




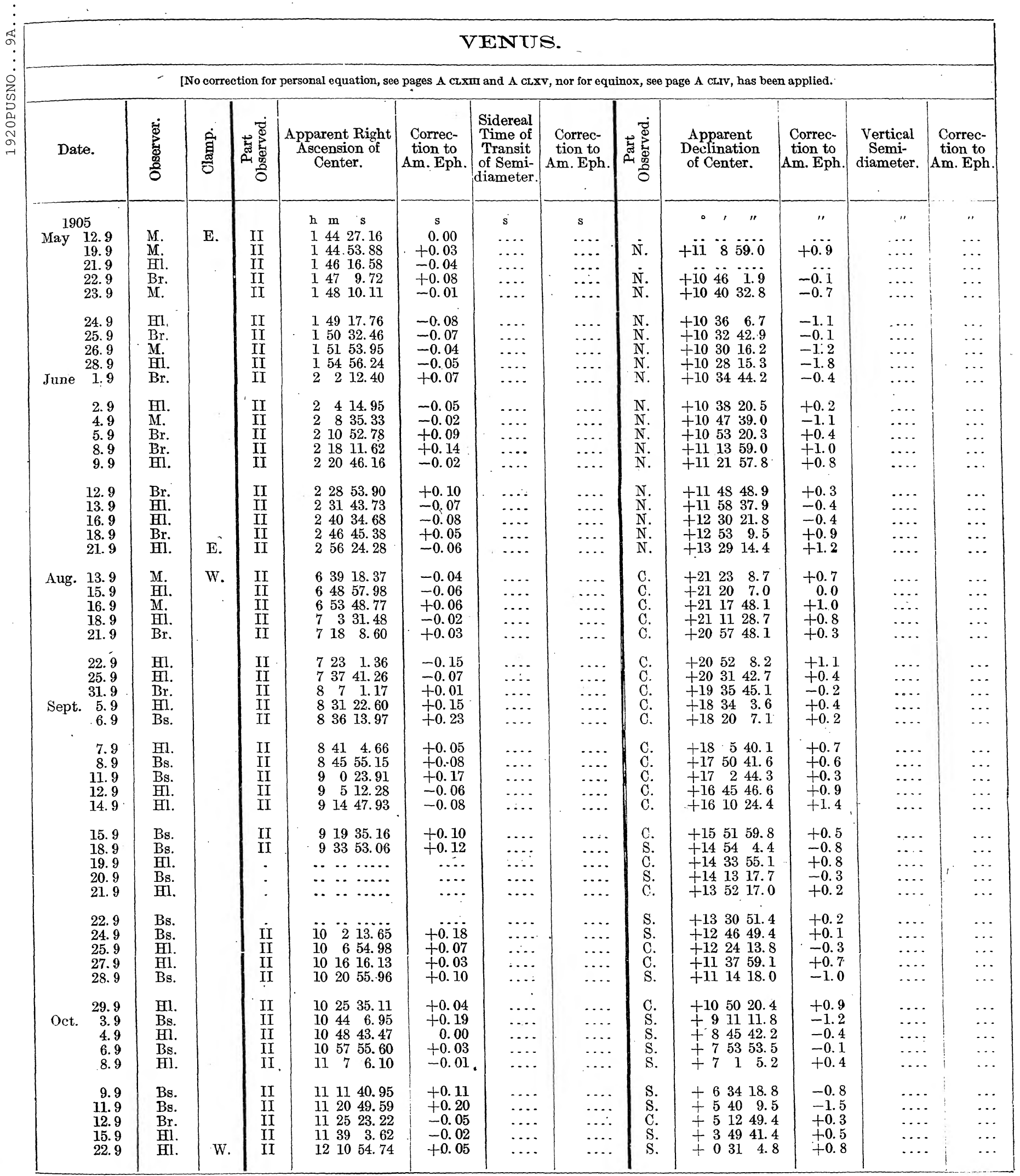




\begin{tabular}{|c|c|c|c|c|c|c|c|c|c|c|c|c|}
\hline \multicolumn{13}{|c|}{ VENUS. } \\
\hline \multicolumn{13}{|c|}{ [No correction for personal equation, see pages A cLxir and A cLxv, nor for equinox, see page A crrv, has been applied.] } \\
\hline Date. & 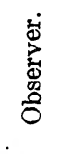 & 空 & 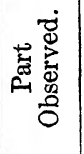 & $\begin{array}{l}\text { Apparent Right } \\
\text { Ascension of } \\
\text { Center. }\end{array}$ & $\begin{array}{c}\text { Correc- } \\
\text { tion to } \\
\text { Am. Eph. }\end{array}$ & $\mid \begin{array}{c}\text { Sidereal } \\
\text { Time of } \\
\text { Transit } \\
\text { of Semi- } \\
\text { diameter. }\end{array}$ & $\begin{array}{c}\text { Correc- } \\
\text { tion to } \\
\text { Am. Eph. }\end{array}$ & 语总 & $\begin{array}{l}\text { Apparent } \\
\text { Declination } \\
\text { of Center. }\end{array}$ & $\begin{array}{c}\text { Correc- } \\
\text { tion to } \\
\text { Am. Eph. }\end{array}$ & \begin{tabular}{|c|} 
Vertieal \\
Semi- \\
diameter.
\end{tabular} & $\begin{array}{l}\text { Correc- } \\
\text { tion to } \\
\text { Am. Eph. }\end{array}$ \\
\hline $\begin{array}{r}1905 \\
\text { Oct:: } 27.9 \\
30.9 \\
31.9 \\
\text { Nov. } \begin{array}{r}1.9 \\
2.9\end{array}\end{array}$ & $\begin{array}{l}\text { Bs. } \\
\text { Br. } \\
\text { Bs. } \\
\text { Hi. } \\
\text { Br. }\end{array}$ & W. & $\begin{array}{l}\text { II } \\
\text { II } \\
\text { II } \\
\text { II } \\
\text { II }\end{array}$ & $\begin{array}{ccr}\mathrm{h} & \mathrm{m} & \mathrm{s} \\
12 & 33 & 42.03 \\
12 & 47 & 25.14 \\
12 & 52 & 0.34 \\
12 & 56 & 35.70 \\
13 & 1 & 11.57\end{array}$ & $\begin{array}{l}\text { s } \\
+0.09 \\
+0.03 \\
+0.17 \\
+0.06 \\
+0.02\end{array}$ & $\begin{array}{l}\mathrm{s} \\
\cdots \\
\cdots \\
\cdots \\
\cdots \\
\cdots\end{array}$ & $\begin{array}{l}\mathrm{s} \\
\cdots \\
\cdots \\
\cdots \\
\cdots \\
\cdots\end{array}$ & $\begin{array}{l}\text { S. } \\
\text { C. } \\
\text { S. } \\
\text { S. } \\
\text { C. }\end{array}$ & 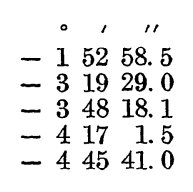 & $\begin{array}{l}-1.3 \\
+0.2 \\
-1.7 \\
-0.8 \\
+0.5\end{array}$ & $\begin{array}{l}\prime \prime \\
\cdots \\
\cdots \\
\cdots \\
\cdots \\
\cdots\end{array}$ & $\begin{array}{l}1 \\
\cdots \\
\cdots \\
\cdots \\
\cdots\end{array}$ \\
\hline $\begin{array}{r}3.9 \\
9.9 \\
10.9 \\
14.9 \\
16.9\end{array}$ & $\begin{array}{l}\text { Bs. } \\
\text { Bs. } \\
\text { Hil. } \\
\text { Bs. } \\
\text { Br. }\end{array}$ & - & $\begin{array}{l}\text { II } \\
\text { II } \\
\text { II } \\
\text { II } \\
\text { II }\end{array}$ & $\begin{array}{rrr}13 & 5 & 47.99 \\
13 & 33 & 38.31 \\
13 & 38 & 18.98 \\
13 & 57 & 9.93 \\
14 & 6 & 40.56\end{array}$ & $\begin{array}{l}+0.06 \\
+0.11 \\
+0.05 \\
+0.12 \\
+0.04\end{array}$ & $\begin{array}{l}\ldots . \\
\cdots \\
\cdots \\
\cdots \\
\cdots\end{array}$ & $\begin{array}{l}\cdots \\
\cdots \\
\cdots \\
\cdots \\
\cdots\end{array}$ & $\begin{array}{l}\text { s. } \\
\text { S. } \\
\text { S. } \\
\text { c. }\end{array}$ & 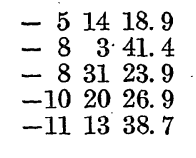 & $\begin{array}{l}-0.9 \\
-1.7 \\
-0.2 \\
-0.5 \\
+0.1\end{array}$ & $\begin{array}{l}\cdots \\
\cdots \\
\cdots \\
\cdots \\
\cdots\end{array}$ & \begin{tabular}{l|}
$\cdots$ \\
$\cdots$ \\
$\cdots$ \\
$\cdots$
\end{tabular} \\
\hline $\begin{array}{r}20.9 \\
21.9 \\
22.9 \\
26.9 \\
\quad 30.9\end{array}$ & $\begin{array}{l}\text { Br. } \\
\text { Bs. } \\
\text { Hl. } \\
\text { Hl. } \\
\text { Br. }\end{array}$ & & $\begin{array}{l}\text { II } \\
\text { II } \\
\text { II } \\
\text { II } \\
\text { II }\end{array}$ & $\begin{array}{lll}14 & 25 & 53.66 \\
14 & 30 & 44.68 \\
14 & 35 & 36.53 \\
14 & 55 & 15.47 \\
15 & 15 & 12.77\end{array}$ & $\begin{array}{l}+0.01 \\
+0.17 \\
+0.08 \\
+0.12 \\
+0.07\end{array}$ & $\begin{array}{l}\cdots \\
\cdots \\
\cdots \\
\cdots \\
\cdots\end{array}$ & $\begin{array}{l}\cdots \\
\cdots \\
\cdots \\
\cdots \\
\cdots\end{array}$ & $\begin{array}{l}\text { c. } \\
\text { c. } \\
\text { c. } \\
\text { c. }\end{array}$ & 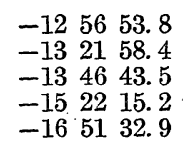 & $\begin{array}{l}+0.2 \\
+0.1 \\
+0.3 \\
+0.3 \\
+0.4\end{array}$ & $\begin{array}{l}\cdots \\
\cdots \\
\cdots \\
\cdots \\
\cdots\end{array}$ & $\begin{array}{l}\cdots \\
\cdots \\
\cdots \\
\cdots\end{array}$ \\
\hline $\begin{array}{r}3.9 \\
4.9 \\
5.9 \\
6.9 \\
7.9\end{array}$ & $\begin{array}{l}\mathrm{Hl} . \\
\text { Br. } \\
\text { Bs. } \\
\text { Hl. } \\
\text { Br. }\end{array}$ & & $\begin{array}{l}\text { II } \\
\text { II } \\
\text { C. } \\
\text { II } \\
\text { II }\end{array}$ & $\begin{array}{llll}15 & 30 & 23.11 \\
15 & 35 & 29.04 \\
15 & 40 & 36.44 \\
15 & 45 & 44.38 \\
15 & 50 & 53.62\end{array}$ & $\begin{array}{l}+0.01 \\
+0.08 \\
+0.43 \\
+0.14 \\
-0.04\end{array}$ & $\begin{array}{l}\cdots \\
\cdots \\
\cdots \\
\cdots \\
\cdots\end{array}$ & $\begin{array}{l}\cdots \\
\cdots \\
\cdots \cdot \\
\cdots \\
\cdots\end{array}$ & $\begin{array}{l}\text { C. } \\
\text { C. } \\
\text { C. } \\
\text { C. } \\
\text { C. }\end{array}$ & $\begin{array}{rrr}-17 & 53 & 54.3 \\
-18 & 13 & 45.2 \\
-18 & 33 & 5.2 \\
-18 & 51 & 55.0 \\
-19 & 10 & 15.6\end{array}$ & $\begin{array}{l}+0.5 \\
+0.1 \\
+1.0 \\
+1: 7 \\
+0.4\end{array}$ & $\begin{array}{l}\ldots \\
\cdots \\
\cdots \\
\cdots \\
\cdots\end{array}$ & $\begin{array}{l}\cdots \\
\cdots \\
\cdots \\
\cdots\end{array}$ \\
\hline $\begin{array}{r}10.9 \\
12.0 \\
13.0 \\
11.0 \\
22.0\end{array}$ & $\begin{array}{l}\text { H1. } \\
\text { Br: } \\
\text { Bs. } \\
\text { Bs. } \\
\text { Bs. }\end{array}$ & & $\begin{array}{l}\text { II } \\
\text { II } \\
\text { II } \\
\text { II } \\
\text { II }\end{array}$ & $\begin{array}{rrr}16 & 6 & 29.00 \\
16 & 11 & 43.00 \\
16 & 16 & 58.31 \\
16 & 43 & 30.02 \\
17 & 5 & 0.44\end{array}$ & $\begin{array}{l}+0.07 \\
+0.03 \\
+0.18 \\
+0.17 \\
+0.02\end{array}$ & $\begin{array}{l}\cdots \\
\cdots \\
\cdots \\
\cdots \\
\cdots\end{array}$ & $\begin{array}{l}\cdots \\
\cdots \\
\cdots \cdot \\
\cdots \\
\cdots\end{array}$ & $\begin{array}{l}\text { C. } \\
\text { C. } \\
\text { c. } \\
\text { C. } \\
\text { c. }\end{array}$ & $\begin{array}{rrr}-20 & 1 & 59.3 \\
-20 & 18 & 5.9 \\
-20 & 33 & 39.2 \\
-21 & 42 & 20.0 \\
-22 & 25 & 51.2\end{array}$ & $\begin{array}{l}+0.3 \\
+1.2 \\
+0.8 \\
+1.7 \\
+1.4\end{array}$ & $\begin{array}{l}\ldots . . \\
\cdots . \\
\cdots \\
\cdots\end{array}$ & $\begin{array}{l}\cdots \\
\cdots \\
\cdots \\
\cdots\end{array}$ \\
\hline $\begin{array}{r}26.0 \\
28.0 \\
30.0 \\
1906\end{array}$ & $\begin{array}{l}\text { Hl. } \\
\text { H1. } \\
\text { Hil. }\end{array}$ & & $\begin{array}{l}\text { II } \\
\text { II } \\
\text {.II }\end{array}$ & $\begin{array}{lll}17 & 26 & 43.37 \\
17 & 37 & 38.35 \\
17 & 48 & 35.14\end{array}$ & $\begin{array}{l}+0.05 \\
+0.01 \\
+0.01\end{array}$ & $\begin{array}{l}\cdots \\
\cdots \\
\cdots\end{array}$ & $\begin{array}{l}\cdots \\
\cdots \\
\cdots\end{array}$ & $\begin{array}{l}\text { c. } \\
\text { c. }\end{array}$ & $\begin{array}{rrr}-22 & 58 & 37.4 \\
-23 & 10 & 49.3 \\
-23 & 20 & 9.8\end{array}$ & $\begin{array}{l}+0.4 \\
+0.4 \\
+1.3\end{array}$ & $\begin{array}{l}\cdots \\
\cdots \\
\cdots\end{array}$ & $\begin{array}{l}\cdots \\
\cdots \\
\cdots\end{array}$ \\
\hline Jan. $\quad 5.0$ & $\mathrm{Br}$ & & II & 182131.25 & -0.01 & $\cdots$ & … & c. & -233053.3 & +1.5 & $\because \cdot$ & ... \\
\hline $\begin{array}{r}\text { Mar. } 22.0 \\
23.0 \\
\text { Apr. } 2.0 \\
12.0 \\
13.0\end{array}$ & $\begin{array}{l}\text { Bs. } \\
\text { Br. } \\
\text { Bs. } \\
\text { Bs. } \\
\text { Br. }\end{array}$ & & I & $\begin{array}{llll}0 & 39 & 11.87 \\
0 & 43 & 44.39 \\
1 & 29 & 27.02 \\
2 & 16 & 6.24 \\
2 & 20 & 50.52\end{array}$ & $\begin{array}{l}+0.06 \\
-0.02 \\
+0.06 \\
+0.07 \\
-0.05\end{array}$ & $\begin{array}{l}0.33 \\
0.41 \\
0.42 \\
0.36\end{array}$ & $\begin{array}{l}-0.01 \\
+0.07 \\
+0.07 \\
+0.01\end{array}$ & $\begin{array}{l}\text { C. } \\
\text { C. } \\
\text { C. } \\
\text { C. }\end{array}$ & $\begin{array}{r}+25720.6 \\
+32749.7 \\
+8271.7 \\
+136652.4 \\
+133317.4\end{array}$ & $\begin{array}{l}+0.7 \\
+0.4 \\
+0.8 \\
+1.0 \\
+0.7\end{array}$ & $\begin{array}{l}\cdots . \\
\cdots \\
\cdots \\
\cdots \\
\cdots\end{array}$ & $\begin{array}{l}\cdots \\
\cdots \\
\cdots \\
\cdots\end{array}$ \\
\hline $\begin{array}{r}17.0 \\
18.0 \\
28.0 \\
30.1 \\
\text { May } \quad 4.1\end{array}$ & $\begin{array}{l}\text { Br. } \\
\text { Bs. } \\
\text { Bs. } \\
\text { Bs. } \\
\text { Br. }\end{array}$ & & $\therefore$ & $\begin{array}{rrr}2 & 39 & 57.81 \\
2 & 44 & 47.27 \\
3 & 34 & 0.01 \\
3 & 44 & 3.73 \\
4 & 4 & 23.99\end{array}$ & $\begin{array}{l}-0.04 \\
+0.07 \\
+0.04 \\
+0.03 \\
+0.03\end{array}$ & $\begin{array}{l}0.38 \\
0.34 \\
0.35 \\
0.31 \\
0.33\end{array}$ & $\begin{array}{l}+0.03 \\
-0.01 \\
-0.02 \\
-0.07 \\
-0.05\end{array}$ & $\begin{array}{l}\text { C. } \\
\text { c. } \\
\text { c. } \\
\text { c. } \\
\text { C. }\end{array}$ & 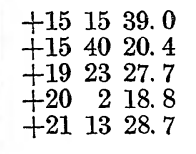 & $\begin{array}{l}-0.7 \\
+0.3 \\
-0.4 \\
-0.1 \\
+0.6\end{array}$ & $\begin{array}{l}\cdots . \\
\cdots \\
\cdots \\
\cdots \\
\cdots\end{array}$ & $\begin{array}{l}\cdots \\
\cdots \\
\cdots \\
\cdots\end{array}$ \\
\hline $\begin{array}{r}\text { June } 15.1 \\
21.1 \\
25.1 \\
27.1 \\
29.1\end{array}$ & $\begin{array}{l}\text { Br. } \\
\text { Hi. } \\
\text { Hil. } \\
\text { Bs. } \\
\text { Br. }\end{array}$ & & $\begin{array}{l}\dot{I} \\
\text { I }\end{array}$ & $\begin{array}{lrr}7 & 45 & 32.57 \\
8 & 16 & 0.53 \\
8 & 35 & 53.20 \\
8 & 45 & 41.44 \\
8 & 55 & 23.37\end{array}$ & $\begin{array}{l}-0.08 \\
-0.12 \\
-0.13 \\
+0.17 \\
-0.08\end{array}$ & $\begin{array}{l}0.45 \\
0.41 \\
\ldots \ldots \\
0.0 \\
0.49\end{array}$ & $\begin{array}{r}+0.01 \\
-0.04 \\
\ldots \ldots \\
+0.02\end{array}$ & $\begin{array}{l}\text { c. } \\
\text { c. } \\
\text { c.. } \\
\text { c. } \\
\text { c. }\end{array}$ & $\begin{array}{rrr}+23 & 1 & 128.3 \\
+21 & 38 & 26.3 \\
+20 & 31 & 14.4 \\
+19 & 54 & 21.8 \\
+19 & 15 & 23.2\end{array}$ & $\begin{array}{r}0.0 \\
-0.1 \\
-0.6 \\
+0.4 \\
+0.2\end{array}$ & $\begin{array}{l}\cdots \\
\cdots \\
\cdots \\
\cdots \\
\cdots\end{array}$ & $\begin{array}{l}\cdots \\
\cdots \\
\cdots \\
\cdots\end{array}$ \\
\hline $\begin{array}{rr} & 30.1 \\
\text { July } & 2.1 \\
3.1 \\
5.1 \\
6.1 \\
6\end{array}$ & $\begin{array}{l}\text { Bs. } \\
\text { Hi. } \\
\text { Br. } \\
\text { Hil. } \\
\text { Br. }\end{array}$ & & $\begin{array}{l}\text { I } \\
\text { I } \\
\dot{I}\end{array}$ & $\begin{array}{rrr}9 & 0 & 12.38 \\
9 & 9 & 45.71 \\
9 & 14 & 30.16 \\
9 & 23 & 54.55 \\
9 & 28 & 34.64\end{array}$ & $\begin{array}{l}+0.03 \\
-0.01 \\
-0.03 \\
-0.15 \\
-0.13\end{array}$ & $\begin{array}{l}\cdots . . \\
\dddot{0} . \ddot{5} \dot{8} \\
\dddot{0} . \ddot{4} \dot{9}\end{array}$ & $\begin{array}{r}\ldots . \\
+0.01 \\
+0.01\end{array}$ & $\begin{array}{l}\text { c. } \\
\text { c. } \\
\text { c. }\end{array}$ & $\begin{array}{l}+183313.7 \\
+175134.4 \\
+17654.6 \\
+164356.2\end{array}$ & $\begin{array}{r}0.00 \\
+0.2 \\
-0.2 \\
-0.2\end{array}$ & $\begin{array}{l}\cdots \\
\cdots \\
\cdots \\
\cdots \\
\cdots\end{array}$ & $\begin{array}{l}\cdots \\
\cdots \\
\cdots \\
\cdots\end{array}$ \\
\hline $\begin{array}{r}7.1 \\
9.1 \\
\text { Aug. } 21.1 \\
27.1 \\
31.1\end{array}$ & $\begin{array}{l}\text { Bs. } \\
\text { Hl. } \\
\text { Br. } \\
\text { Hli. } \\
\text { Br. }\end{array}$ & W. & $\begin{array}{l}\text { I } \\
\text { I } \\
\text { I } \\
\text { I }\end{array}$ & $\begin{array}{rrrr}9 & 33 & 13.30 \\
9 & 42 & 26.21 \\
12 & 42 & 58.61 \\
13 & 6 & 35.42 \\
13 & 22 & 14.72\end{array}$ & $\begin{array}{l}-0.08 \\
-0.10 \\
-0.03 \\
-0.12 \\
-0.04\end{array}$ & $\begin{array}{l}0.45 \\
\ldots . \\
\cdots \\
\cdots\end{array}$ & $\begin{array}{c}-0.03 \\
\cdots \\
\cdots \\
\cdots \\
\cdots\end{array}$ & $\begin{array}{l}\text { C. } \\
\text { C. } \\
\text { C. } \\
\text { N. } \\
\text { C. }\end{array}$ & $\begin{array}{r}+16 \quad 2033.2 \\
+15 \quad 3234.7 \\
-513 \quad 5.3 \\
-8128.9 \\
-10 \quad 829.2\end{array}$ & $\begin{array}{l}+0.1 \\
-0.1 \\
+1.5 \\
-1.3 \\
-0.2\end{array}$ & $\begin{array}{l}\ldots . \\
\cdots \\
\cdots \\
\cdots \\
\cdots \\
\cdots\end{array}$ & $\begin{array}{l}\cdots \\
\cdots \\
\cdots \\
\cdots \\
\cdots\end{array}$ \\
\hline
\end{tabular}




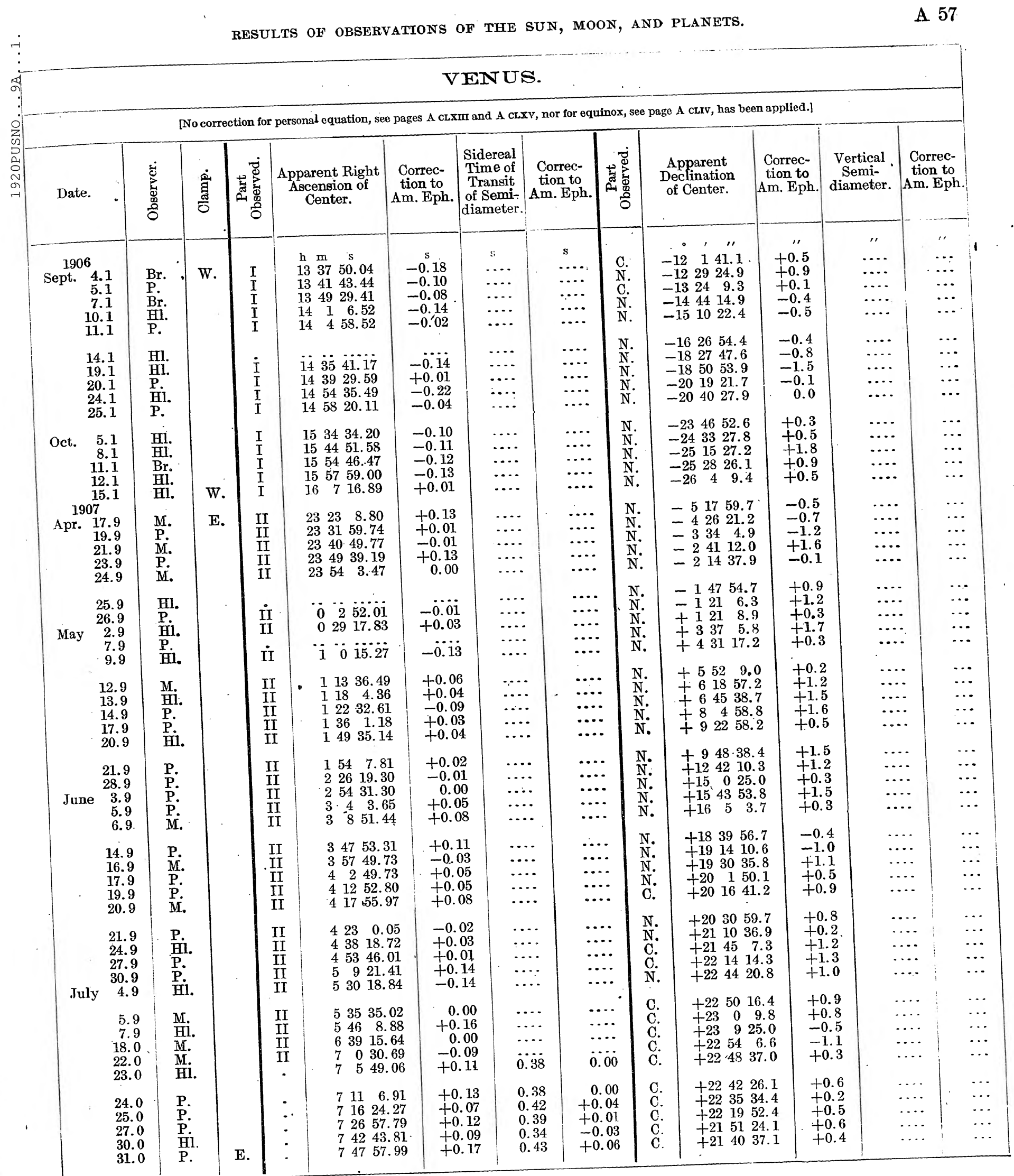




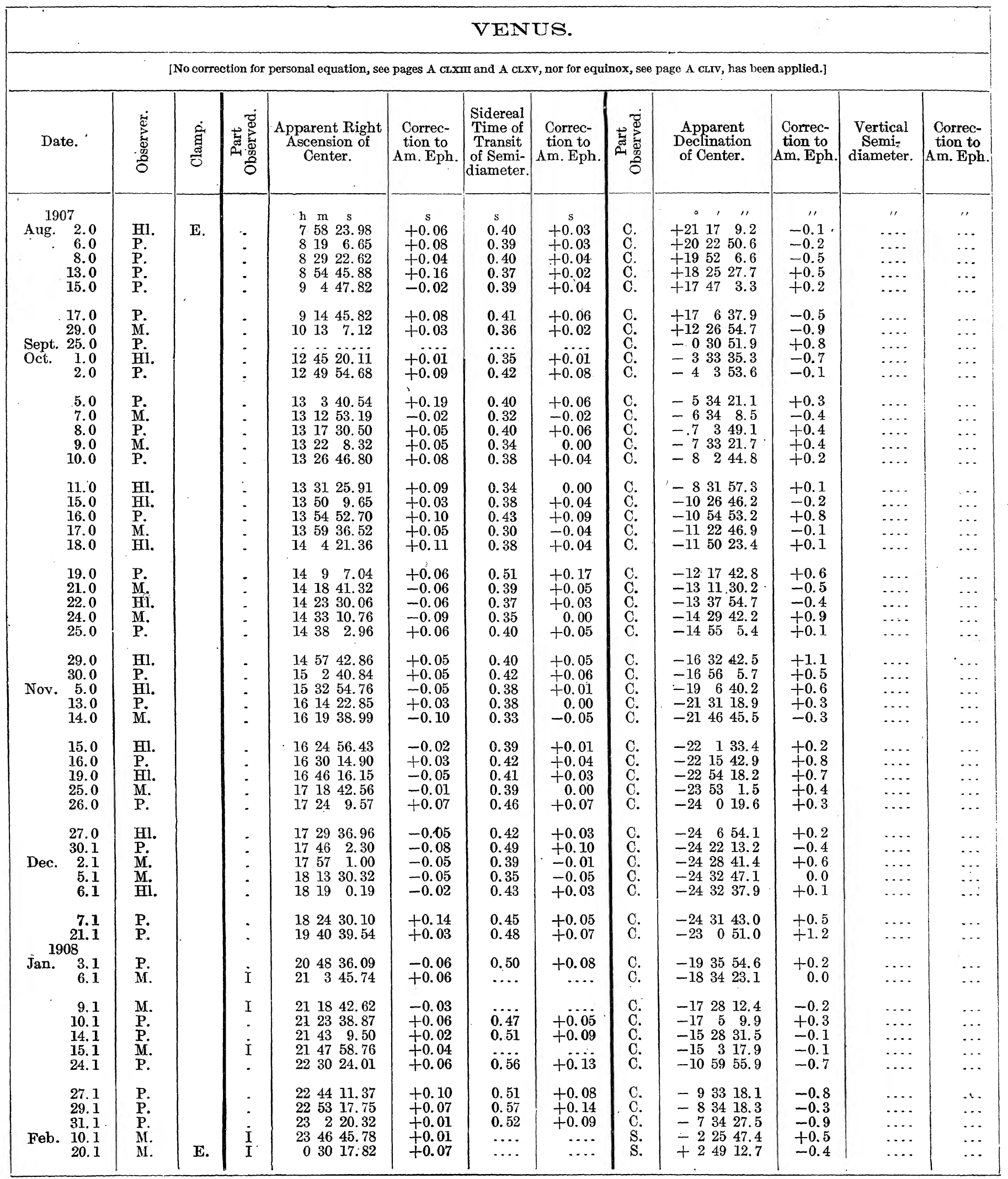


Results of observations of THE SUN, MOON, AND PLANets.

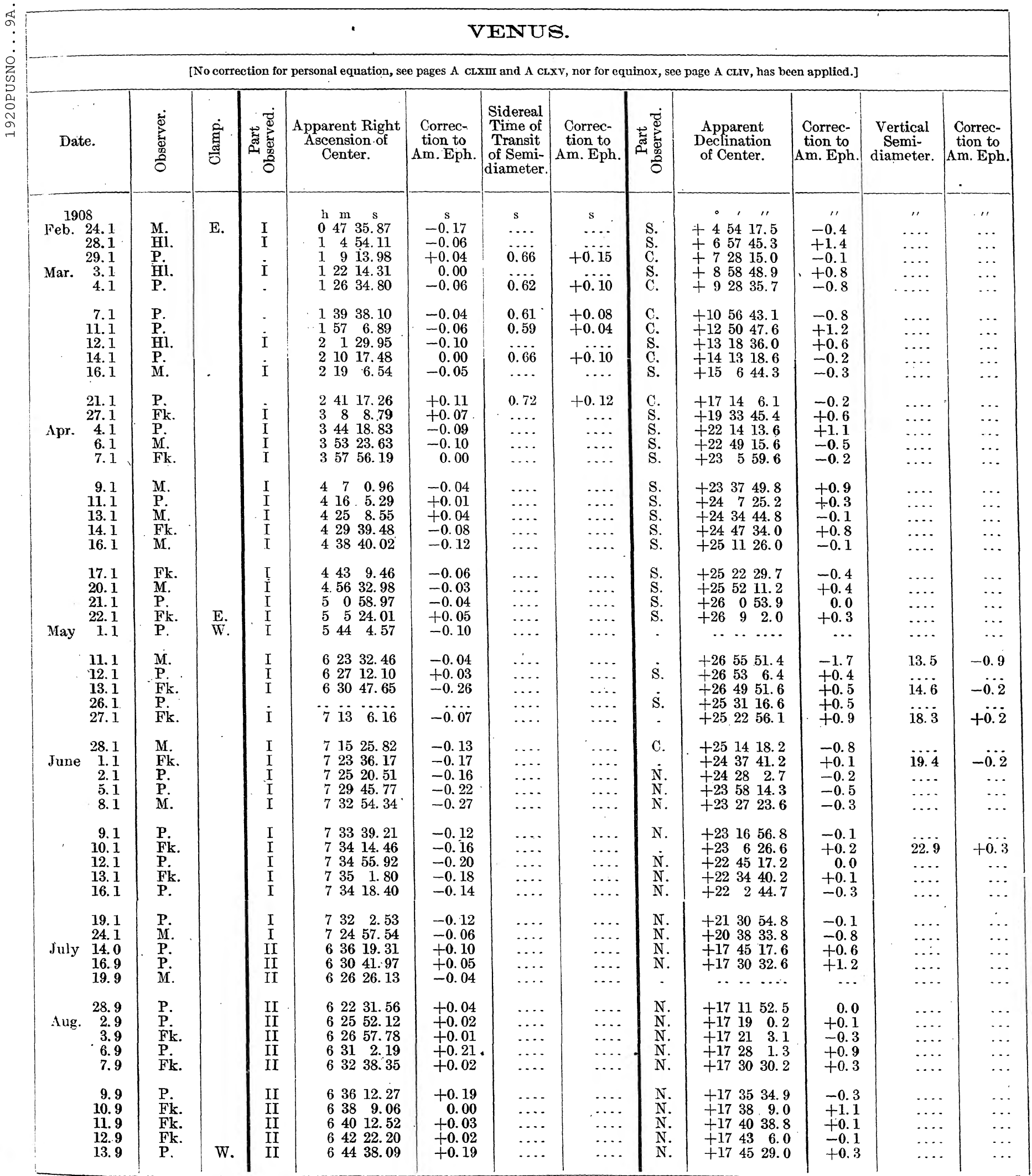




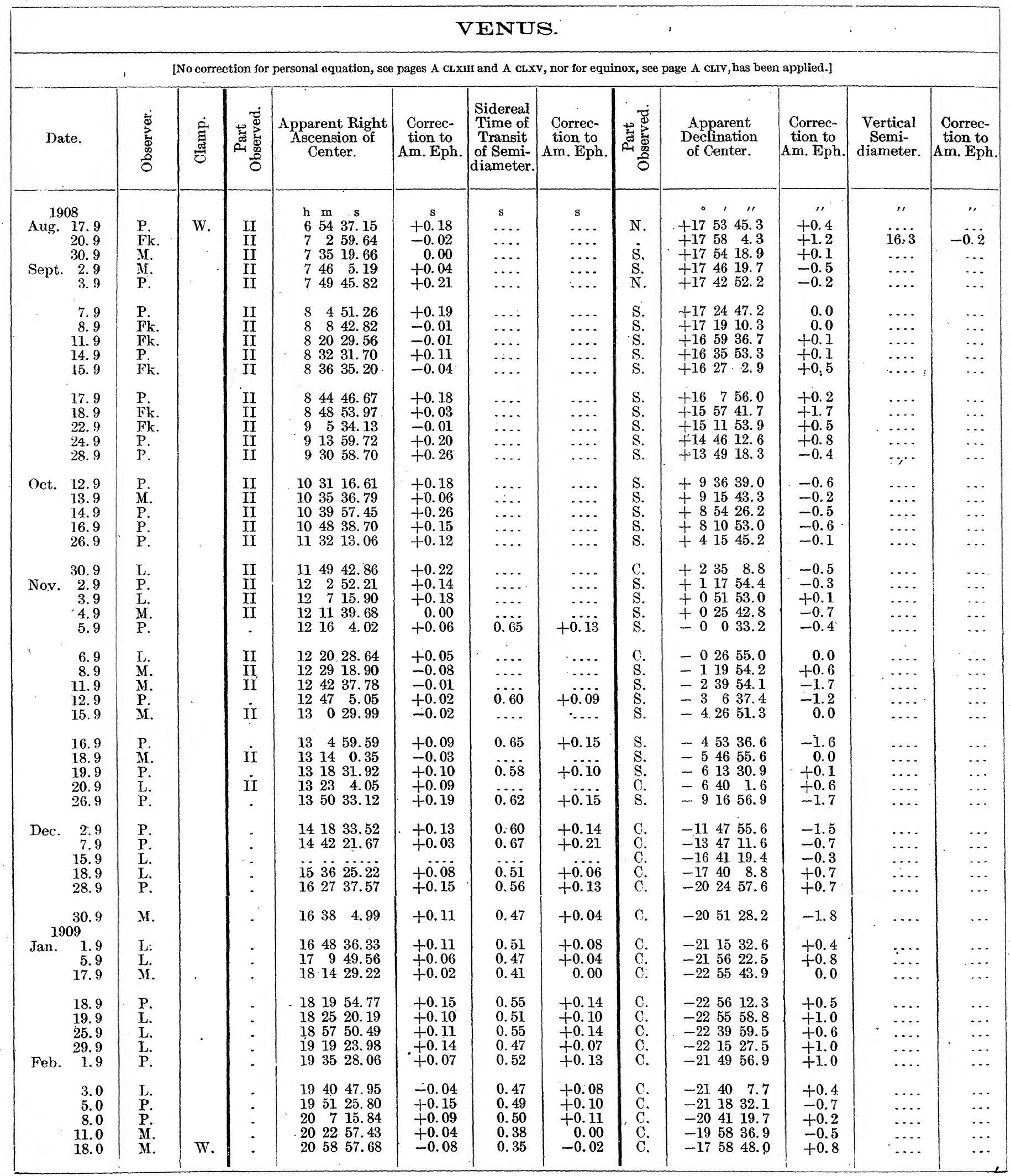


RESUlts OF OBSERVATIONS OF THE SUN, MOON, ANU PLANETS.

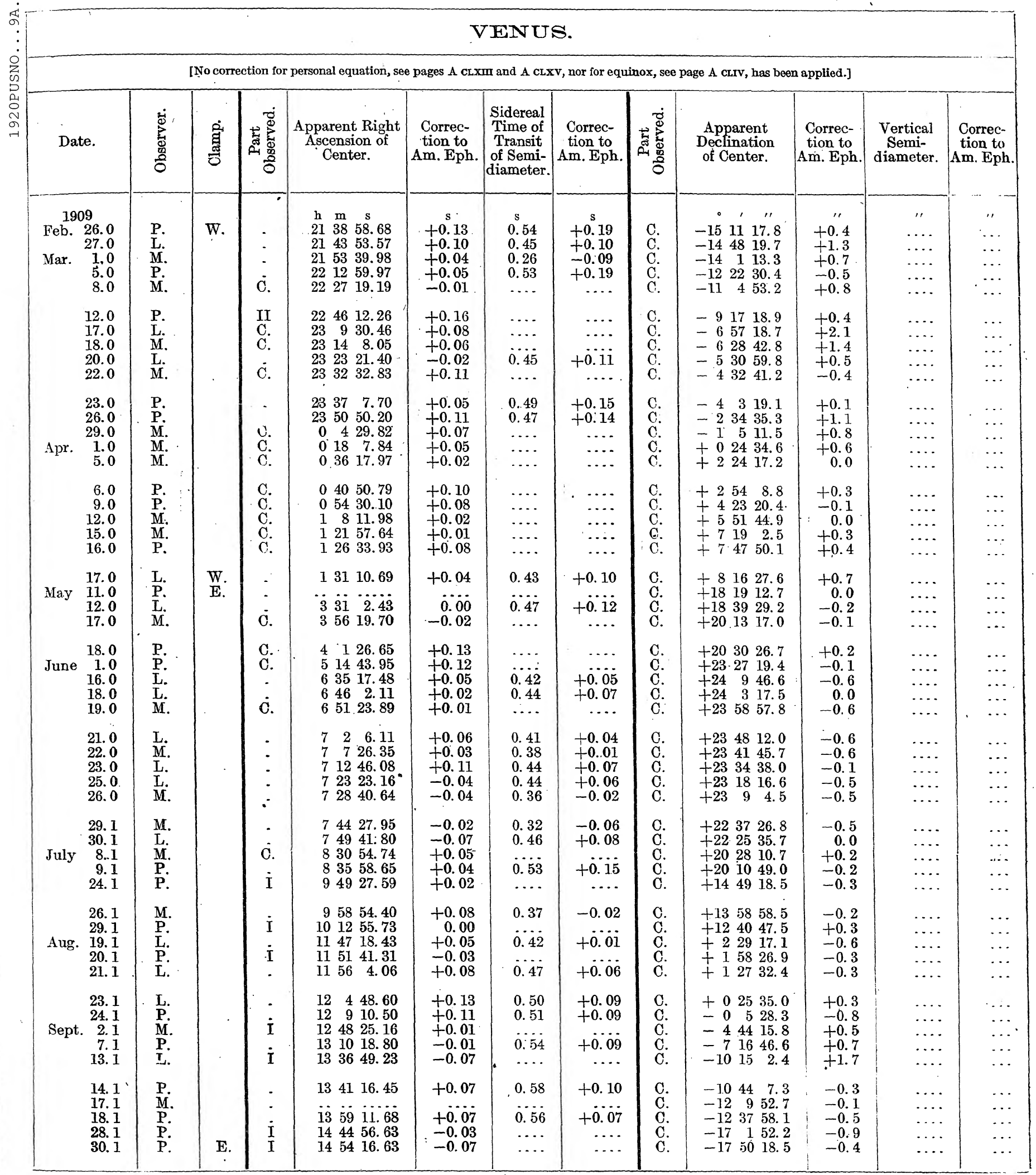




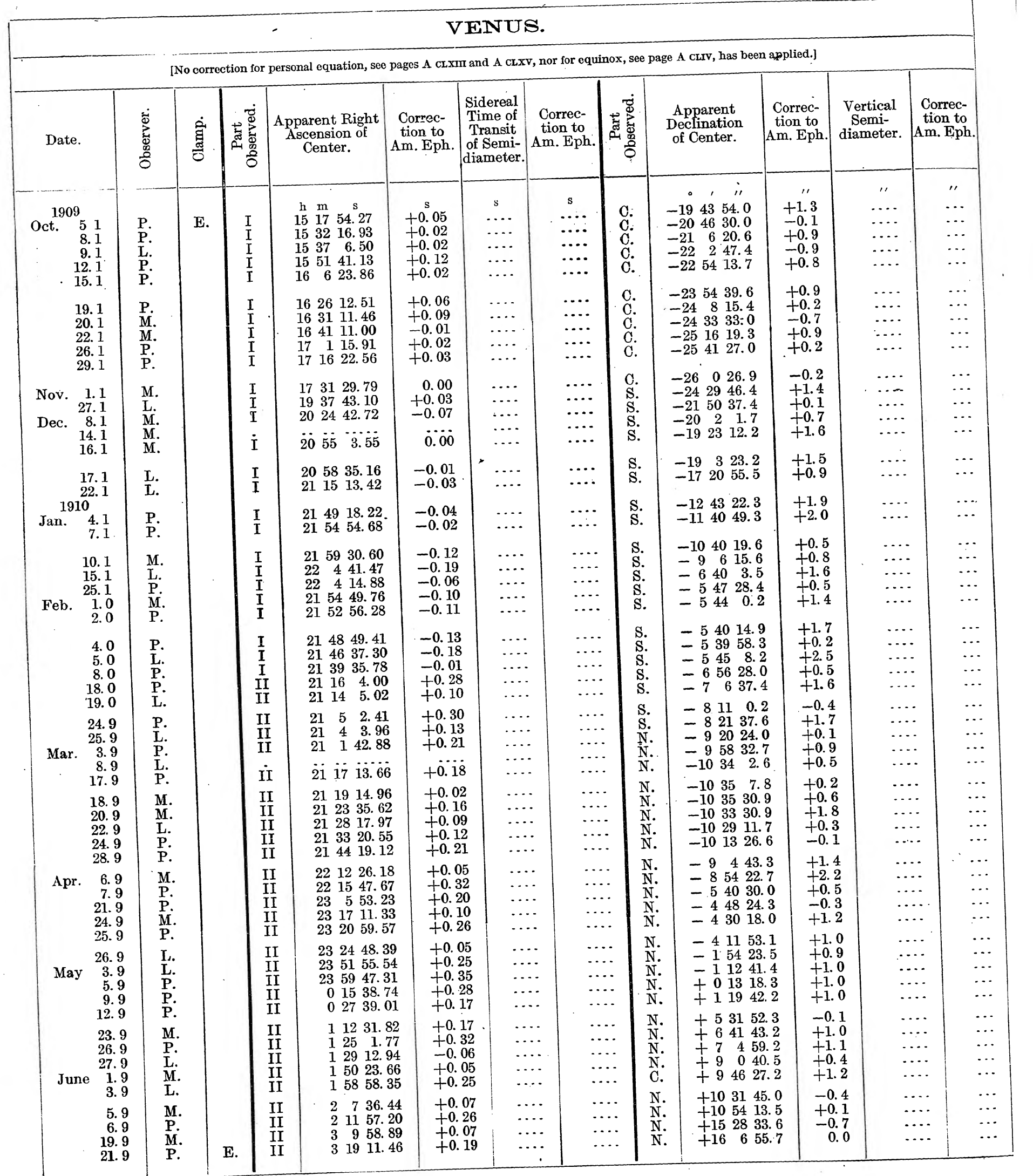




\begin{tabular}{|c|c|c|c|c|c|c|c|c|c|c|c|c|c|c|c|}
\hline \multicolumn{16}{|c|}{ MARS. } \\
\hline \multicolumn{16}{|c|}{ [No correction for personal equation, see pages A cLxur and A CLxv, nor for equinox, see page A CLrv, has been applied.] } \\
\hline \multirow{2}{*}{\multicolumn{2}{|c|}{ Date. }} & \multirow{2}{*}{ 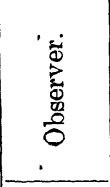 } & \multirow{2}{*}{ 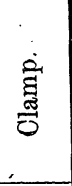 } & \multirow{2}{*}{ 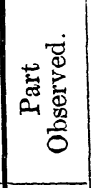 } & \multirow{2}{*}{$\begin{array}{c}\text { Apparent } \\
\text { Right } \\
\text { Ascension of } \\
\text { Center. }\end{array}$} & \multicolumn{2}{|c|}{ Correction to- } & \multirow{2}{*}{$\begin{array}{l}\text { Sidereal } \\
\text { Time of } \\
\text { Transit } \\
\text { of Semi- } \\
\text { diameter. }\end{array}$} & \multirow{2}{*}{$\begin{array}{c}\text { Correc- } \\
\text { tion } \\
\text { to Am. } \\
\text { Eph. }\end{array}$} & \multirow{2}{*}{ 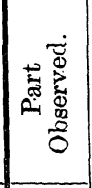 } & \multirow{2}{*}{$\begin{array}{c}\text { Apparent } \\
\text { Declination } \\
\text { of Center. }\end{array}$} & \multicolumn{2}{|c|}{ Correction to- } & \multirow{2}{*}{$\begin{array}{c}\text { Vertical } \\
\text { Semi- } \\
\text { diameter. }\end{array}$} & \multirow{2}{*}{$\begin{array}{l}\text { Correc- } \\
\text { tion } \\
\text { to Am. } \\
\text { Eph. }\end{array}$} \\
\hline & & & & & & $\begin{array}{l}\text { Am. } \\
\text { Eph. }\end{array}$ & \begin{tabular}{|c|} 
Ross's \\
Tables.
\end{tabular} & & & & & $\begin{array}{l}\text { Am. } \\
\text { Eph. }\end{array}$ & $\begin{array}{c}\text { Ross's } \\
\text { Tables. }\end{array}$ & & \\
\hline $\begin{array}{r}1905 \\
\text { Feb. } \\
1 \\
2 \\
2\end{array}$ & \begin{tabular}{r|}
5 \\
6.7 \\
9.7 \\
17.7 \\
20.7 \\
23.7
\end{tabular} & $\begin{array}{l}\text { Br. } \\
\text { Br. } \\
\text { M. } \\
\text { Br. } \\
\text { Br. }\end{array}$ & E. & : & $\begin{array}{|rrr|}\mathrm{h} & \mathrm{m} & \mathrm{s} \\
14 & 38 & 24.47 \\
14 & 43 & 18.38 \\
14 & 55 & 38.85 \\
14 & 59 & 58.52 \\
15 & 4 & 6.94\end{array}$ & $\begin{array}{c}\mathrm{s} \\
-0.09 \\
+0.03 \\
-0.05 \\
-0.03 \\
-0.11\end{array}$ & $\begin{array}{c}\mathrm{s} \\
+0.01 \\
+0.13 \\
+0.06 \\
+0.09 \\
+0.02\end{array}$ & $\begin{array}{l}\mathrm{s} \\
0.43 \\
0.40 \\
0.38 \\
0.42 \\
0.38\end{array}$ & $\begin{array}{c}s \\
+0.15 \\
+0.11 \\
+0.07 \\
+0.11 \\
+0.06\end{array}$ & $\begin{array}{l}\text { C. } \\
\text { C. } \\
\text { C. } \\
\text { C. } \\
\text { C. }\end{array}$ & $\begin{array}{rcc}\circ & . & \prime \prime \\
-13 & 36 & 57.6 \\
-13 & 59 & 47.9 \\
-14 & 55 & 32.8 \\
-15 & 14 & 33.1 \\
-15 & 32 & 28.5\end{array}$ & $\begin{array}{r} \\
+1.0 \\
+1.0 \\
+0.2 \\
+1.3 \\
+0.3 \\
+0.7\end{array}$ & $\begin{array}{c}\prime \prime \\
+0.6 \\
-0.3 \\
+0.9 \\
-0.2 \\
+0.2\end{array}$ & $\begin{array}{l}\prime \prime \\
\cdots \\
\cdots \\
\cdots \\
\cdots \\
\cdots\end{array}$ & $\begin{array}{l}\prime \prime \\
\ldots \\
\cdots \\
\cdots \\
\cdots \\
\cdots\end{array}$ \\
\hline Mar. $\begin{array}{r}2 \\
1 \\
1 \\
1\end{array}$ & $\begin{array}{r}24.7 \\
1.7 \\
10.7 \\
12.7 \\
13.7\end{array}$ & $\begin{array}{l}\text { M. } \\
\text { Y. } \\
\text { M. } \\
\text { Y. } \\
\text { Br. }\end{array}$ & ' & $\begin{array}{l}. \\
. \\
.\end{array}$ & $\begin{array}{rrr}15 & 5 & 27.12 \\
15 & 11 & 46.15 \\
15 & 21 & 23.10 \\
15 & 23 & 10.53 \\
15 & 24 & 0.99\end{array}$ & $\begin{array}{l}-0.10 \\
-0.03 \\
-0.09 \\
-0.04 \\
-0.16\end{array}$ & $\begin{array}{r}+0.03 \\
+0.10 \\
+0.07 \\
+0.12 \\
0.00\end{array}$ & $\begin{array}{l}0.30 \\
0.39 \\
0.39 \\
0.38 \\
0.44\end{array}$ & $\begin{array}{r}-0.03 \\
+0.04 \\
0.00 \\
-0.02 \\
+0.04\end{array}$ & $\begin{array}{l}\text { C. } \\
\text { C. } \\
\text { C. } \\
\text { C. } \\
\text { C. }\end{array}$ & $\begin{array}{|rrr|}-15 & 38 & 12.9 \\
-16 & 5 & 6.4 \\
-16 & 45 & 53.8 \\
-16 & 53 & 36.9 \\
-16 & 57 & 17.3\end{array}$ & $\begin{array}{l}+0.7 \\
+1.4 \\
+1.0 \\
+1.4 \\
+1.8\end{array}$ & $\begin{array}{l}+0.2 \\
+0.8 \\
+0.3 \\
+0.7 \\
+1.1\end{array}$ & \begin{tabular}{l|}
$\cdots$ \\
$\cdots$ \\
$\cdots$ \\
$\cdots$ \\
$\cdots$
\end{tabular} & $\begin{array}{l}\cdots \\
\cdots \\
\cdots \\
\cdots \\
\cdots\end{array}$ \\
\hline & $\begin{array}{l}15.7 \\
25.6 \\
26.6 \\
27.6 \\
28.6\end{array}$ & $\begin{array}{l}\text { Y. } \\
\text { Br. } \\
\text { Y. } \\
\text { Br. } \\
\text { M. }\end{array}$ & & $\therefore$ & $\begin{array}{rrr}15 & 25 & 35.87 \\
15 & 31 & 9.28 \\
15 & 31 & 28.66 \\
15 & 31 & 44.99 \\
15 & 31 & 58.69\end{array}$ & $\begin{array}{l}-0.04 \\
-0.19 \\
-0.08 \\
-0.24 \\
-0.20\end{array}$ & $\begin{array}{r}+0.12 \\
0.00 \\
+0.11 \\
-0.05 \\
0.00\end{array}$ & $\begin{array}{l}0.40 \\
0.45 \\
0.48 \\
0.54 \\
0.41\end{array}$ & $\begin{array}{l}-0.01 \\
-0.01 \\
+0.01 \\
+0.07 \\
-0.07\end{array}$ & $\begin{array}{l}\text { C. } \\
\text { C.: } \\
\text { C. } \\
\text { C. } \\
\text { c. }\end{array}$ & $\mid \begin{array}{rrr}-17 & 4 & 17.3 \\
-17 & 31 & 55.5 \\
-17 & 34 & 1.0 \\
-17 & 35 & 58.8 \\
-17 & 37 & 48.1\end{array}$ & $\begin{array}{l}+1.3 \\
+2.1 \\
+1.6 \\
+1.1 \\
+1.5\end{array}$ & $\begin{array}{l}+0.6 \\
+1.3 \\
+0.8 \\
+0.3 \\
+0.7\end{array}$ & \begin{tabular}{l|}
$\cdots$ \\
$\cdots$ \\
$\cdots$ \\
$\cdots$ \\
$\cdots$ \\
\end{tabular} & $\begin{array}{l}\cdots \\
\cdots \\
\cdots \\
\cdots \\
\cdots\end{array}$ \\
\hline Apr. & $\begin{array}{r}30.6 \\
31.6 \\
7.6 \\
9.6 \\
13.6\end{array}$ & $\begin{array}{l}\mathrm{Br} . \\
\mathrm{M} . \\
\mathrm{M} . \\
\mathrm{Y} . \\
\mathrm{Br} .\end{array}$ & & : & $\begin{array}{lll}15 & 32 & 17.34 \\
15 & 32 & 22.34 \\
15 & 31 & 31.33 \\
1.5 & 30 & 48.67 \\
15 & 28 & 45.54\end{array}$ & $\begin{array}{l}-0.23 \\
-0.17 \\
-0.20 \\
-0.19 \\
-0.25\end{array}$ & $\begin{array}{r}-0.03 \\
+0.04 \\
+0.03 \\
+0.04 \\
0.00\end{array}$ & $\begin{array}{l}0.53 \\
0.44 \\
0.56 \\
0.51 \\
0.56\end{array}$ & $\begin{array}{r}+0.04 \\
-0.05 \\
+0.03 \\
-0.03 \\
0.00\end{array}$ & $\begin{array}{l}\text { c. } \\
\text { c. } \\
\text { c. } \\
\text { c. } \\
\text { c. }\end{array}$ & $\begin{array}{|rrr|}-17 & 41 & 2.8 \\
-17 & 42 & 29.8 \\
-17 & 48 & 44.9 \\
-17 & 49 & 10.7 \\
-17 & 48 & 43.8\end{array}$ & $\begin{array}{r}+2.5 \\
+1.5 \\
+2.2 \\
{[+9.7]} \\
+1.9\end{array}$ & $\mid \begin{array}{c}+1.6 \\
+0.6 \\
+1.2 \\
{[+8.75} \\
+0.8\end{array}$ & $\begin{array}{l}\cdots \\
\cdots \\
\cdots \\
\cdots \\
\cdots\end{array}$ & $\begin{array}{l}\cdots \\
\cdots \\
\cdots \\
\cdots \\
\cdots\end{array}$ \\
\hline May $\frac{1}{1}$ & $\begin{array}{l}18.6 \\
12.5 \\
16.5 \\
20.5 \\
21.4\end{array}$ & $\begin{array}{l}\text { M. } \\
\text { Y. } \\
\text { Br. } \\
\text { M. } \\
\text { Br. }\end{array}$ & & : & $\begin{array}{rrr}15 & 25 & 2.38 \\
14 & 53 & 50.00 \\
14 & 47 & 55.22 \\
14 & 42 & 18.09 \\
14 & 40 & 57.59\end{array}$ & $\begin{array}{l}-0.32 \\
-0.35 \\
-0.35 \\
-0.23 \\
-0.34\end{array}$ & $\begin{array}{l}-0.06 \\
-0.06 \\
-0.06 \\
+0.06 \\
-0.06\end{array}$ & $\begin{array}{l}0.56 \\
0.62 \\
0.73 \\
0.64 \\
0.72\end{array}$ & $\begin{array}{l}-0.02 \\
-0.03 \\
+0.08 \\
-0.01 \\
+0.07\end{array}$ & $\begin{array}{l}\text { c. } \\
\text { C. } \\
\text { C. } \\
\text { C. }\end{array}$ & $\begin{array}{rrr}-17 & 44 & 50.3 \\
-16 & 42 & 1.5 \\
-16 & 27 & 47.8 \\
-16 & 14 & 6.8 \\
-16 & 10 & 50.4\end{array}$ & $\begin{array}{l}+1.2 \\
+2.3 \\
+2.4 \\
+2.4 \\
+3.5\end{array}$ & $\begin{array}{r}0.0 \\
+0.7 \\
+0.8 \\
+0.7 \\
+1.8\end{array}$ & $\begin{array}{l}\cdots \\
\cdots \\
\cdots \\
\cdots \\
\cdots\end{array}$ & $\begin{array}{l}\cdots \\
\cdots \\
\cdots \\
\cdots \\
\cdots\end{array}$ \\
\hline June & $\begin{array}{r}23.4 \\
27.4 \\
1.4 \\
2.4 \\
9.4 \\
7\end{array}$ & $\begin{array}{l}\mathrm{Br} . \\
\mathrm{M} . \\
\mathrm{Ei} .-\mathrm{Y} . \\
\mathrm{Br} . \\
\mathrm{Br} .\end{array}$ & & 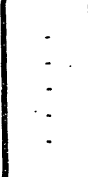 & $\begin{array}{lll}14 & 38 & 22.49 \\
14 & 33 & 38.73 \\
14 & 28 & 43.05 \\
14 & 27 & 52.72 \\
14 & 23 & 29.96\end{array}$ & $\begin{array}{l}-0.32 \\
-0.32 \\
-0.26 \\
-0.25 \\
-0.25\end{array}$ & $\begin{array}{r}-0.04 \\
-0.05 \\
0.00 \\
+0.01 \\
-0.01\end{array}$ & $\begin{array}{l}0.70 \\
0.61 \\
0.59 \\
0.62 \\
0.62\end{array}$ & $\begin{array}{l}+0.05 \\
-0.03 \\
-0.04 \\
-0.01 \\
+0.02\end{array}$ & $\begin{array}{l}\text { C. } \\
\text { C. } \\
\text { C. } \\
\text { C. } \\
\text { C. }\end{array}$ & $\left|\begin{array}{rrr}-16 & 4 & 36.4 \\
-15 & 53 & 30.7 \\
-15 & 42 & 58.7 \\
-15 & 41 & 24.5 \\
-15 & 36 & 8.0\end{array}\right|$ & $\begin{array}{l}+3.3 \\
+2.7 \\
+3.4 \\
+3.2 \\
+2.9\end{array}$ & $\begin{array}{r}+1.6 \\
+1.0 \\
+1.7 \\
+1.5 \\
+1.3\end{array}$ & $\begin{array}{l} \\
\cdots \\
\cdots \\
\cdots \\
\cdots \\
\cdots\end{array}$ & $\begin{array}{l} \\
\cdots \\
\cdots \\
\cdots \\
\cdots\end{array}$ \\
\hline $\begin{array}{l}\text { May } \\
\text { June }\end{array}$ & $\begin{array}{r}20.6 \\
29.6 \\
3.6 \\
16.6 \\
17.6\end{array}$ & $\begin{array}{l}\mathrm{H} . \\
\mathrm{M} . \\
\mathrm{P} . \\
\mathrm{M} . \\
\mathrm{P} .\end{array}$ & & $\begin{array}{l}: \\
:\end{array}$ & $\begin{array}{lll}19 & 17 & 24.65 \\
19 & 22 & 34.04 \\
19 & 23 & 42.83 \\
19 & 20 & 20.53 \\
19 & 19 & 42.26\end{array}$ & $\begin{array}{l}-0.18 \\
-0.22 \\
-0.21 \\
-0.22 \\
-0.29\end{array}$ & $\begin{array}{l}+0.03 \\
+0.01 \\
+0.03 \\
+0.05 \\
-0.02\end{array}$ & $\begin{array}{l}0.62 \\
0.69 \\
0.71 \\
0.81 \\
0.78\end{array}$ & $\begin{array}{r}+0.01 \\
+0.02 \\
0.00 \\
-0.01 \\
-0.05\end{array}$ & $\begin{array}{l}\text { C. } \\
\text { C. } \\
\text { C. } \\
\text { C. } \\
\text { C. }\end{array}$ & $\begin{array}{rrr}-24 & 24 & 54.6 \\
-24 & 50 & 13.6 \\
-25 & 9 & 2.3 \\
-26 & 12 & 47.5 \\
-26 & 18 & 21.9\end{array}$ & $\begin{array}{r}+1.4 \\
+0.7 \\
-0.2 \\
-0.2 \\
0.0\end{array}$ & $\begin{array}{l}+1.5 \\
+0.8 \\
-0.1 \\
-0.1 \\
+0.1\end{array}$ & $\begin{array}{l}\cdots \\
\cdots \\
\cdots \\
\cdots \\
\cdots\end{array}$ & $\begin{array}{l}\cdots \\
\cdots \\
\cdots \\
\cdots\end{array}$ \\
\hline July & $\begin{array}{r}26.5 \\
30.5 \\
3.5 \\
7.5 \\
21.4\end{array}$ & $\begin{array}{l}\text { Hूl. } \\
\text { P. } \\
\text { P. } \\
\text { Hil. } \\
\text { Hl: }\end{array}$ & & : & $\begin{array}{rrr}19 & 11 & 51.30 \\
19 & 7 & 20.41 \\
19 & 3 & 39.43 \\
18 & 58 & 30.53 \\
18 & 41 & 30.66\end{array}$ & $\begin{array}{l}-0.31 \\
-0.19 \\
-0.23 \\
-0.37 \\
-0.23\end{array}$ & $\begin{array}{l}-0.02 \\
+0.10 \\
+0.06 \\
-0.08 \\
+0.04\end{array}$ & $\begin{array}{l}0.88 \\
0.88 \\
0.96 \\
0.89 \\
0.86\end{array}$ & $\begin{array}{l}-0.01 \\
-0.02 \\
+0.04 \\
-0.04 \\
-0.07\end{array}$ & $\begin{array}{l}\text { c. } \\
\text { C. } \\
\text { c. } \\
\text { c. } \\
\text { c. }\end{array}$ & $\mid \begin{array}{rrr}-27 & 9 & 47.5 \\
-27 & 31 & 58.5 \\
-27 & 47 & 36.4 \\
-28 & 6 & 31.3 \\
-28 & 48 & 17.7\end{array}$ & $\begin{array}{r}+1.2 \\
0.0 \\
+0.5 \\
0.0 \\
+1.3\end{array}$ & $\begin{array}{r}+1.3 \\
+0.1 \\
+0.5 \\
0.0 \\
+1.1\end{array}$ & $\begin{array}{l}\cdots \\
\cdots \\
\cdots \\
\cdots \\
\cdots\end{array}$ & $\begin{array}{l}\cdots \\
\cdots \\
\cdots \\
\cdots \\
\cdots\end{array}$ \\
\hline Aug. & $\begin{array}{l}23.4 \\
27.4 \\
29.4 \\
30.4 \\
29.3\end{array}$ & $\begin{array}{l}\mathrm{Hl} . \\
\mathrm{P} . \\
\mathrm{M} . \\
\mathrm{Hi} . \\
\mathrm{M} .\end{array}$ & & i & $\begin{array}{llll}18 & 39 & 34.22 \\
18 & 36 & .15 .58 \\
18 & 34 & 54.89 \\
18 & 34 & 19.57 \\
18 & 44 & 45.41\end{array}$ & $\begin{array}{r}-0.22 \\
-0.16 \\
-0.24 \\
-0.25 \\
0.00\end{array}$ & $\begin{array}{r}+0.05 \\
+0.10 \\
+0.01 \\
0.00 \\
+0.14\end{array}$ & $\begin{array}{l}0.89 \\
0.87 \\
0.83 \\
0.90 \\
\cdots\end{array}$ & $\begin{array}{r}-0.04 \\
-0.04 \\
-0.07 \\
0.00 \\
\cdots\end{array}$ & $\begin{array}{l}\text { c. } \\
\text { c. } \\
\text { C. } \\
\text { c. } \\
\text { c. }\end{array}$ & $\begin{array}{llll}-28 & 50 & 52.6 \\
-28 & 53 & 38.7 \\
-28 & 53 & 53.5 \\
-28 & 53 & 44.8 \\
-27 & 49 & 31.1\end{array}$ & $\begin{array}{l}+1.3 \\
+0.9 \\
+1.1 \\
+1.4 \\
+1.0\end{array}$ & $\begin{array}{l}+1.1 \\
+0.7 \\
+0.9 \\
+1.2 \\
+0.9\end{array}$ & $\begin{array}{l}\cdots \\
\cdots \\
\cdots \\
\cdots \\
\cdots\end{array}$ & $\begin{array}{l}\cdots \\
\cdots \\
\cdots \\
\cdots \\
\cdots\end{array}$ \\
\hline Sept. & $\begin{array}{r}7.3 \\
10.3 \\
12.3 \\
13.3 \\
14.3\end{array}$ & $\begin{array}{l}\text { M. } \\
\text { Ei.-M. } \\
\text { M. } \\
\text { Hi.-M. } \\
\text { P.-M. }\end{array}$ & & $\therefore$ & $\begin{array}{rrr}18 & 57 & 15.61 \\
19 & 2 & 10.08 \\
19 & 5 & 37.41 \\
19 & 7 & 24.25 \\
19 & 9 & 13.26\end{array}$ & $\begin{array}{l}+0.02 \\
-0.04 \\
-0.12 \\
-0.13 \\
+0.03\end{array}$ & $\begin{array}{l}+0.13 \\
+0.06 \\
-0.02 \\
-0.04 \\
+0.12\end{array}$ & $\begin{array}{l}0.69 \\
0.54 \\
0.65 \\
0.53 \\
0.69\end{array}$ & $\begin{array}{l}+0.04 \\
-0.09 \\
+0.03 \\
-0.08 \\
+0.08\end{array}$ & $\begin{array}{l}\text { C. } \\
\text { C. } \\
\text { C. } \\
\text { C. } \\
\text { C. }\end{array}$ & $\mid \begin{array}{rrr}-27 & 12 & 3.7 \\
-26 & 57 & 34.1 \\
-26 & 47 & 18.3 \\
-26 & 42 & 0.5 \\
-26 & 36 & 32.8\end{array}$ & $\begin{array}{l}+1.2 \\
+1.9 \\
+2.2 \\
+1.1 \\
+2.5\end{array}$ & $\begin{array}{l}+1.1 \\
+1.8 \\
+2.1 \\
+1.0 \\
+2.4\end{array}$ & $\begin{array}{l}\cdots \\
\cdots \\
\cdots \\
\cdots \\
\cdots\end{array}$ & $\begin{array}{l}\cdots \\
\cdots \\
\cdots \\
\cdots \\
\cdots\end{array}$ \\
\hline 190 & 16.3 & M. & & $\cdot$ & $\begin{array}{lll}19 & 12 & 56.57\end{array}$ & -0.11 & -0.02 & 0.57 & -0.03 & C. & $-26 \quad 25 \quad 18.3$ & +1.4 & +1.3 & $\because \cdots$ & $\cdots$ \\
\hline Aug. & $\begin{array}{l}19.6 \\
21.6 \\
22.6\end{array}$ & $\begin{array}{l}\mathrm{L} . \\
\mathrm{L} . \\
\mathrm{P} .\end{array}$ & E. & . & \begin{tabular}{lll|}
0 & 33 & 18.38 \\
0 & 33 & 33.02 \\
0 & 33 & 35.63
\end{tabular} & $\begin{array}{l}+0.12 \\
+0.10 \\
+0.15\end{array}$ & $\begin{array}{l}-0.03 \\
-0.06 \\
-0.01\end{array}$ & $\begin{array}{l}0.83 \\
0.83 \\
0.83\end{array}$ & $\begin{array}{l}+0.08 \\
+0.07 \\
+0.07\end{array} \mid$ & $\begin{array}{l}\text { C. } \\
\text { C. }\end{array}$ & $\left|\begin{array}{rrr}-2 & 17 & 13.9 \\
-2 & 18 & 14.6 \\
-2 & 19 & 7.6\end{array}\right|$ & $\begin{array}{l}+1.2 \\
+2.0 \\
+2.5\end{array}$ & $\begin{array}{l}-0.1 \\
+0.7 \\
+1.1\end{array}$ & . & $\begin{array}{l}\cdots \\
\cdots \\
\cdots\end{array}$ \\
\hline
\end{tabular}

$29297^{\circ}-20-34$ 


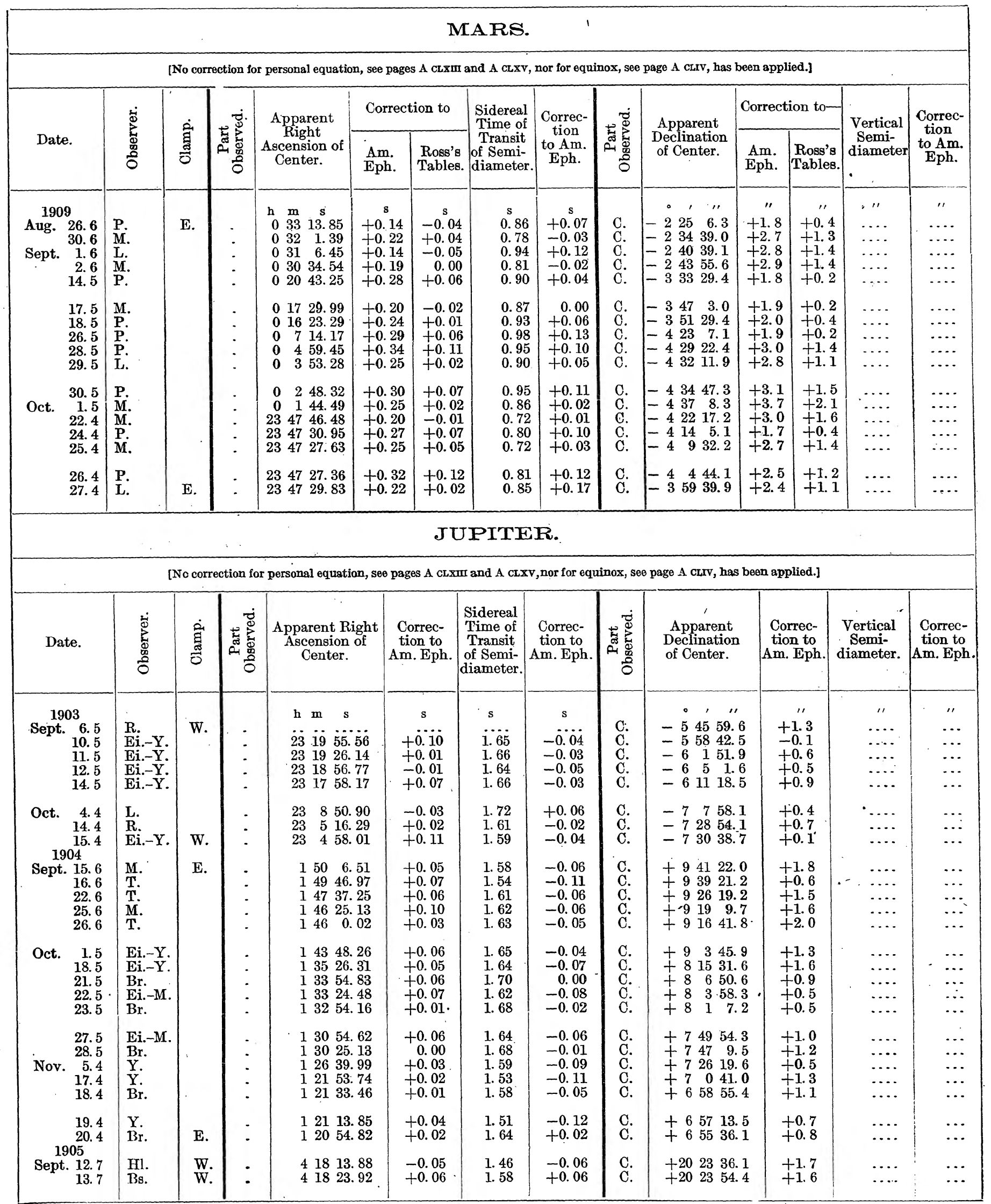


RESUlts of OBSERVATIONS OF THE SUN, MOON, AND PLANETS.

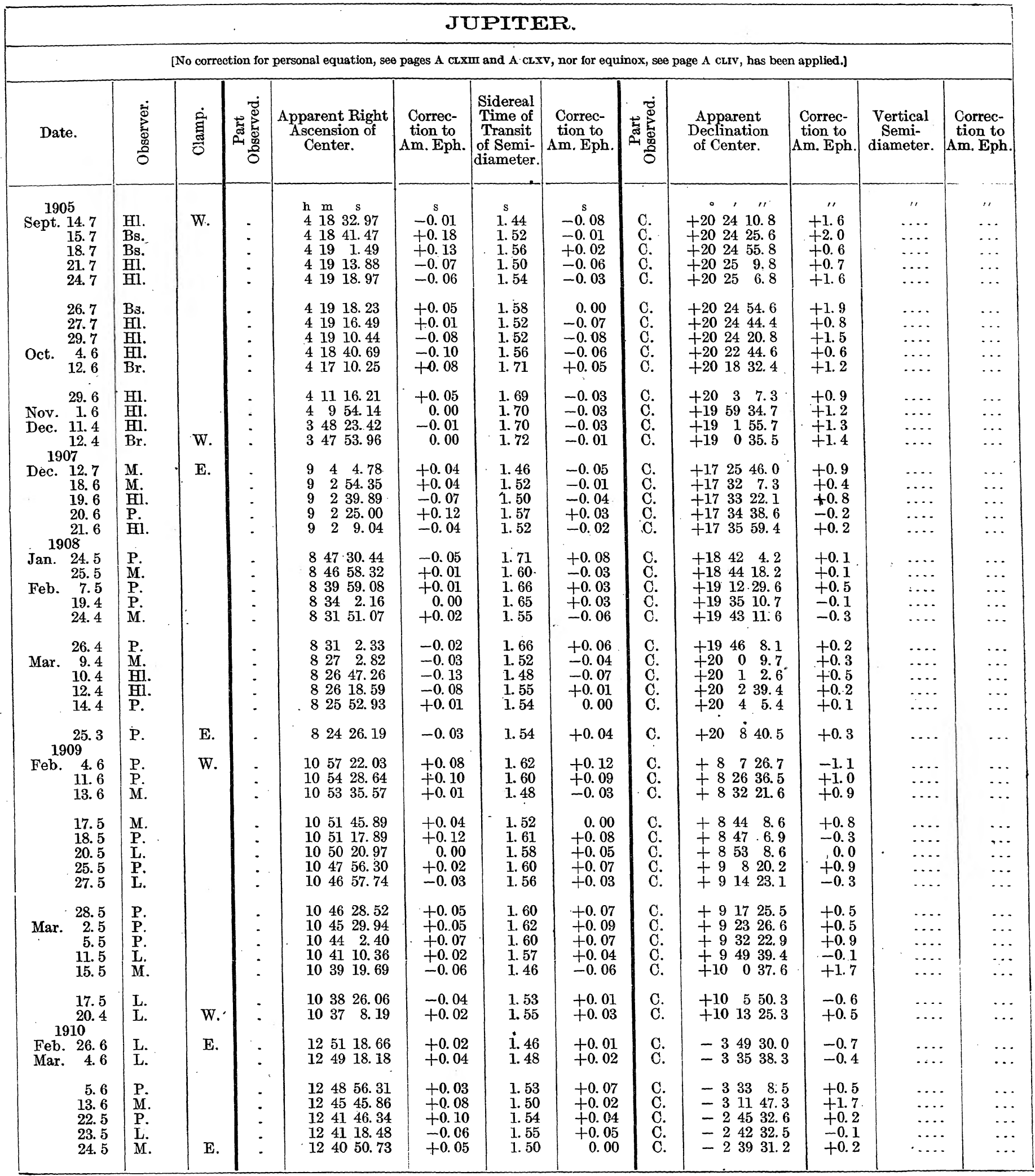




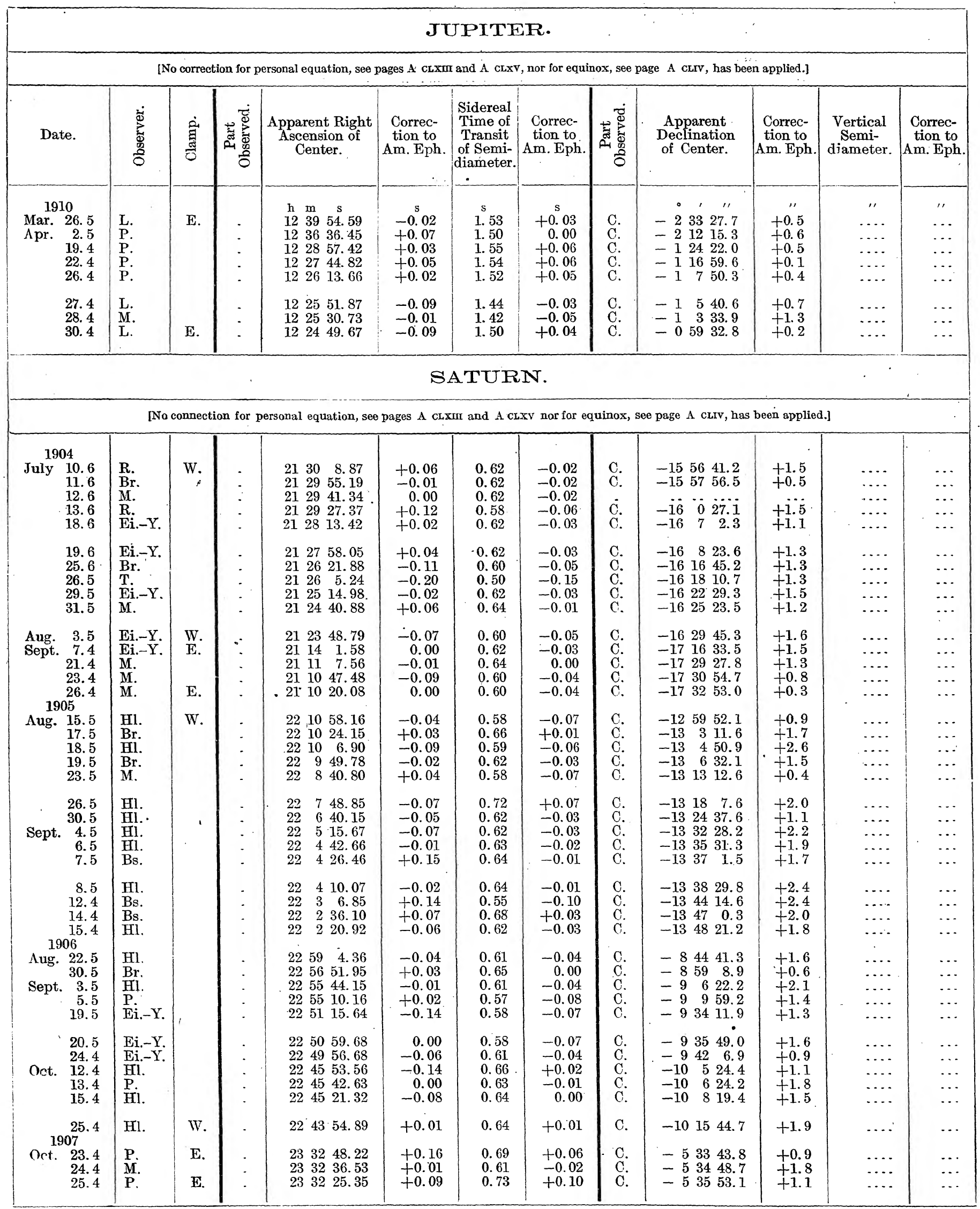


RESUlts of observations of THE SUN, MOON, AND PLANETS.

\begin{tabular}{|c|c|c|c|c|c|c|c|c|c|c|c|c|}
\hline \multicolumn{13}{|c|}{ SATURN. } \\
\hline \multicolumn{13}{|c|}{ [No correction for personal equation, see pages A cLXIr and A cLXv, nor for equinox, see page A cLIV, has been applied.] } \\
\hline Date. & 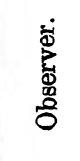 & 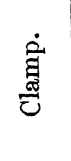 & 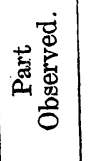 & $\begin{array}{l}\text { Apparent Right } \\
\text { Ascension of } \\
\text { Center. }\end{array}$ & $\begin{array}{c}\text { Correc- } \\
\text { tion to } \\
\text { Am. Eph. }\end{array}$ & $\begin{array}{l}\text { Sidereal } \\
\text { Time of } \\
\text { Transit } \\
\text { of Semi- } \\
\text { diameter. }\end{array}$ & $\begin{array}{c}\text { Correc- } \\
\text { tion to } \\
\text { Am. Eph. }\end{array}$ & 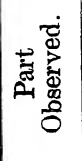 & $\begin{array}{c}\text { Apparent } \\
\text { Declination } \\
\text { of Center. }\end{array}$ & $\begin{array}{c}\text { Correc- } \\
\text { tion to } \\
\text { Am. Eph. }\end{array}$ & \begin{tabular}{c|c} 
Vertical \\
Semi- \\
diameter.
\end{tabular} \mid A & $\begin{array}{c}\text { Correc- } \\
\text { tion to } \\
\text { Am. Eph. }\end{array}$ \\
\hline \begin{tabular}{cc}
\multicolumn{2}{c}{1907} \\
Oct. & 29.4 \\
& 30.4 \\
Nov. & 1.4 \\
& 4.4 \\
& 5.4
\end{tabular} & $\begin{array}{l}\mathrm{Hl} . \\
\text { P. } \\
\text { Hil. } \\
\text { M. } \\
\text { Hi. }\end{array}$ & E. & $\begin{array}{l}1: \\
: \\
:\end{array}$ & $\begin{array}{ccc}\mathrm{h} & \mathrm{m} & \mathrm{s} \\
23 & 31 & 43.28 \\
23 & 31 & 33.62 \\
23 & 31 & 15.10 \\
23 & 30 & 49.90 \\
23 & 30 & 42.11\end{array}$ & $\begin{array}{c}\text { s } \\
+0.03 \\
+0.10 \\
+0.06 \\
+0.07 \\
-0.01\end{array}$ & $\begin{array}{l}\mathrm{s} \\
0.59 \\
0.66 \\
0.58 \\
0.62 \\
0.59\end{array}$ & $\begin{array}{c}\mathrm{s} \\
-0.04 \\
+0.03 \\
-0.05 \\
-0.01 \\
-0.04\end{array}$ & $\begin{array}{l}\text { c. } \\
\text { C. } \\
\text { c. } \\
\text { C. } \\
\text { C. }\end{array}$ & 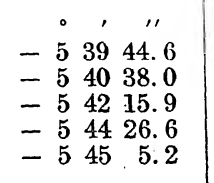 & 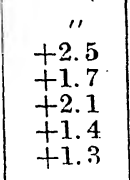 & $\begin{array}{l}\prime \prime \\
\cdots \\
\cdots \\
\cdots \\
\cdots \\
\cdots\end{array}$ & $\begin{array}{l}\prime \prime \\
\cdots \\
\cdots \\
\cdots \\
\cdots\end{array}$ \\
\hline $\begin{array}{r}7.4 \\
14.3 \\
\text { Dec. } 6.3 \\
7.3\end{array}$ & $\begin{array}{l}\text { M. } \\
\text { M. } \\
\text { Hi. } \\
\text { P. }\end{array}$ & E. & $\dot{.}$ & $\begin{array}{lll}23 & 30 & 27.82 \\
23 & 29 & 49.06 \\
23 & 29 & 49.54 \\
23 & 29 & 54.21\end{array}$ & $\begin{array}{l}+0.07 \\
+0.10 \\
-0.04 \\
+0.09\end{array}$ & $\begin{array}{l}0.59 \\
0.60 \\
0.64 \\
0.64\end{array}$ & $\begin{array}{l}-0.03 \\
-0.02 \\
+0.04 \\
+0.04\end{array}$ & $\begin{array}{l}\text { C. } \\
\text { C. } \\
\text { C. } \\
\text { C. }\end{array}$ & $\begin{array}{rrr}- & 546 & 15.1 \\
-5 & 49 & 1.0 \\
-5 & 44 & 10.4 \\
-5 & 4329.6\end{array}$ & $\begin{array}{l}+1.3 \\
+1.4 \\
+1.3 \\
-0.3\end{array}$ & $\begin{array}{l}\cdots \\
\cdots \\
\cdots \\
\cdots\end{array}$ & $\begin{array}{l}\cdots \\
\cdots \\
\cdots\end{array}$ \\
\hline $\begin{array}{r}1908 \\
\text { Aug. } 30.6 \\
31.6 \\
\text { Sept. } 6.6 \\
8.6 \\
11.5\end{array}$ & $\begin{array}{l}\text { M. } \\
\text { P. } \\
\text { P. } \\
\text { F. }\end{array}$ & W. & $\dot{.}$ & $\begin{array}{lll}0 & 36 & 51.49 \\
0 & 36 & 38.45 \\
0 & 35 & 14.85 \\
0 & 34 & 45.28 \\
0 & 33 & 59.28\end{array}$ & $\begin{array}{l}+0.02 \\
+0.10 \\
+0.16 \\
+0.19 \\
0.00\end{array}$ & $\begin{array}{l}0.62 \\
0.70 \\
0.68 \\
0.76 \\
0.66\end{array}$ & $\begin{array}{l}-0.03 \\
+0.05 \\
+0.03 \\
+0.11 \\
+0.01\end{array}$ & $\begin{array}{l}\text { C.. } \\
\text { C. } \\
\text { C. } \\
\text { C. } \\
\text { C. }\end{array}$ & 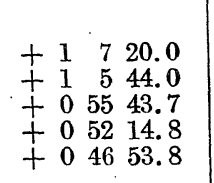 & $\begin{array}{l}+0.7 \\
+0.6 \\
+1.9 \\
+2.1 \\
+1.5\end{array}$ & $\begin{array}{l}\cdots \\
\cdots \\
\cdots \\
\cdots\end{array}$ & $\begin{array}{l}\cdots \\
\cdots \\
\cdots \\
\cdots\end{array}$ \\
\hline $\begin{array}{r}14.5 \\
15.5 \\
16.5 \\
\text { Oct. } 6.5 \\
7.5\end{array}$ & $\begin{array}{l}\text { M. } \\
\text { P. } \\
\text { Fk. } \\
\text { P. } \\
\text { L. }\end{array}$ & & . & $\begin{array}{rrr}0 & 33 & 11.98 \\
0 & 32 & 55.96 \\
0 & 32 & 39.65 \\
0 & 27 & 0.39 \\
0 & 26 & 43.30\end{array}$ & $\begin{array}{r}+0.03 \\
+0.09 \\
0.00 \\
+0.11 \\
+0.02\end{array}$ & $\begin{array}{l}0.62 \\
0.72 \\
0.66 \\
0.70 \\
0.64\end{array}$ & $\begin{array}{r}-0.04 \\
+0.06 \\
0.00 \\
+0.04 \\
-0.02\end{array}$ & $\begin{array}{l}\text { C. } \\
\text { c. } \\
\text { c. } \\
\text { C. } \\
\text { c. }\end{array}$ & $\begin{array}{rrrr}+ & 0 & 41 & 25.5 \\
+ & 0 & 39 & 36.3 \\
+ & 0 & 37 & 45.4 \\
+0 & 0 & 22.9 \\
+ & 0 & 1 & 25.6\end{array}$ & $\begin{array}{l}+0.4 \\
+1.5 \\
+1.6 \\
+1.5 \\
+1.5\end{array}$ & $\begin{array}{l}\cdots \\
\cdots \\
\cdots \\
\cdots\end{array}$ & $\begin{array}{l}\cdots \\
\cdots \\
\cdots \\
\cdots \\
\cdots\end{array}$ \\
\hline $\begin{array}{r}12.5 \\
20.4 \\
26.4 \\
31.4 \\
\text { Nov. } 2.4 \\
1909\end{array}$ & $\begin{array}{l}\text { M. } \\
\text { P. } \\
\text { M. } \\
\text { L. } \\
\text { M. }\end{array}$ & W. & : & $\begin{array}{llr}0 & 25 & 19.54 \\
0 & 23 & 11.54 \\
0 & 21 & 42.81 \\
0 & 20 & 34.94 \\
0 & 20 & 9.70\end{array}$ & $\begin{array}{l}+0.07 \\
+0.11 \\
+0.14 \\
+0.01 \\
+0.07\end{array}$ & $\begin{array}{l}0.66 \\
0.70 \\
0.64 \\
0.68 \\
0.61\end{array}$ & $\begin{array}{r}0.00 \\
+0.05 \\
-0.01 \\
+0.03 \\
-0.04\end{array}$ & $\begin{array}{l}\text { C. } \\
\text { C. } \\
\text { C. } \\
\text { C. } \\
\text { C. }\end{array}$ & 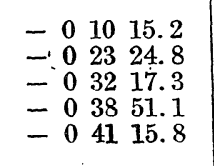 & $\begin{array}{l}+1.6 \\
+1.9 \\
+0.9 \\
+1.4 \\
+0.6\end{array}$ & $\begin{array}{l}\cdots \\
\cdots \\
\cdots \\
\cdots \\
\cdots\end{array}$ & $\begin{array}{l}\cdots \\
\cdots \\
\cdots \\
\cdots\end{array}$ \\
\hline $\begin{array}{r}1909 \\
\text { Sept. } 14.6 \\
17.6 \\
18.6 \\
26.6 \\
28.5\end{array}$ & $\begin{array}{l}\mathrm{P} . \\
\mathrm{M} . \\
\mathrm{P} . \\
\mathrm{P} .\end{array}$ & E. & $\begin{array}{l}\cdot \\
- \\
-\end{array}$ & $\begin{array}{lll}1 & 24 & 56.87 \\
1 & 24 & 14.47 \\
1 & 23 & 59.99 \\
1 & 21 & 55.87 \\
1 & 21 & 23.05\end{array}$ & $\begin{array}{l}+0.08 \\
+0.04 \\
+0.16 \\
+0.21 \\
+0.18\end{array}$ & $\begin{array}{l}0.73 \\
0.64 \\
0.72 \\
0.68 \\
0.72\end{array}$ & $\begin{array}{l}+0.07 \\
+0.03 \\
+0.05 \\
+0.01 \\
+0.05\end{array}$ & $\begin{array}{l}\text { C. } \\
\text { C. } \\
\text { C.: } \\
\text { C. } \\
\text { C. }\end{array}$ & $\begin{array}{r}+55833.2 \\
+55349.9 \\
+55213.1 \\
+53845.9 \\
+53516.6\end{array}$ & $\begin{array}{l}+1.3 \\
+1.3 \\
+1.2 \\
+1.4 \\
+1.5\end{array}$ & $\begin{array}{l}\cdots \\
\cdots \\
\cdots \\
\cdots \\
\cdots\end{array}$ & $\begin{array}{l}\cdots \\
\cdots \\
\cdots \\
\cdots\end{array}$ \\
\hline 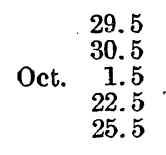 & $\begin{array}{l}\mathrm{L} . \\
\mathrm{P} . \\
\mathrm{M} . \\
\mathrm{M} .\end{array}$ & &. & $\begin{array}{rrr}1 & 21 & 6.28 \\
1 & 20 & 49.64 \\
1 & 20 & 32.74 \\
1 & 14 & 25.49 \\
1 & 13 & 33.97\end{array}$ & $\begin{array}{l}+0.02 \\
+0.13 \\
+0.11 \\
+0.08 \\
+0.04\end{array}$ & $\begin{array}{l}0.70 \\
0.72 \\
0.64 \\
0.65 \\
0.66\end{array}$ & $\begin{array}{l}+0.03 \\
+0.05 \\
-0.03 \\
-0.02 \\
-0.01\end{array}$ & $\begin{array}{l}\text { C. } \\
\text { C. } \\
\text { C. } \\
\text { C. } \\
\text { C. }\end{array}$ & $\begin{array}{r}+53330.6 \\
+53145.3 \\
+52958.7 \\
+45243.3 \\
+44742.2\end{array}$ & $\begin{array}{l}+0.9 \\
+1.6 \\
+1.5 \\
+1.2 \\
+1.2\end{array}$ & $\begin{array}{l}\cdots \\
\cdots \\
\cdots \\
\cdots\end{array}$ & $\begin{array}{l}\cdots \\
\cdots \\
\cdots \\
\cdots \\
\cdots\end{array}$ \\
\hline $\begin{array}{r}26.5 \\
27.5 \\
28.5 \\
29.5 \\
30.5 \\
1910\end{array}$ & $\begin{array}{l}\text { P. } \\
\text { L. } \\
\text { M. } \\
\text { P. }\end{array}$ & & $:$ & $\begin{array}{lll}1 & 13 & 17.10 \\
1 & 13 & 0.23 \\
1 & 12 & 43.54 \\
1 & 12 & 26.92 \\
1 & 12 & 10.63 \\
0 & & \end{array}$ & $\begin{array}{l}+0.11 \\
+0.07 \\
+0.08 \\
+0.02 \\
+0.14\end{array}$ & $\begin{array}{l}0.71 \\
0.72 \\
0.64 \\
0.70 \\
0.75\end{array}$ & $\begin{array}{l}+0.04 \\
+0.05 \\
-0.03 \\
+0.03 \\
+0.08\end{array}$ & $\begin{array}{l}\text { c. } \\
\text { c. } \\
\text { c. } \\
\text { c. }\end{array}$ & $\begin{array}{r}+4463.6 \\
+44426.1 \\
+44250.5 \\
+44114.9 \\
+43941.3\end{array}$ & $\begin{array}{l}+0.9 \\
+0.6 \\
+1.1 \\
+0.5 \\
+0.8\end{array}$ & $\begin{array}{l}\cdots \\
\cdots \\
\cdots \\
\cdots \\
\cdots\end{array}$ & $\begin{array}{l}\cdots \\
\cdots \\
\cdots \\
\cdots\end{array}$ \\
\hline $\begin{aligned} 1910 \\
\text { Sept. } 21.6 \\
22.6 \\
26.6 \\
\text { Oct. } \begin{array}{l}16.5 \\
17.5\end{array}\end{aligned}$ & $\begin{array}{l}\text { M. } \\
\text { L. } \\
\text { P. } \\
\text { P. } \\
\text { M. }\end{array}$ & & : & $\begin{array}{llr}2 & 16 & 54.35 \\
2 & 16 & 41.76 \\
2 & 15 & 48.76 \\
2 & 10 & 26.07 \\
2 & 10 & 8.10\end{array}$ & $\begin{array}{l}+0.08 \\
+0.02 \\
+0.18 \\
+0.09 \\
-0.01\end{array}$ & $\begin{array}{l}0.67 \\
0.68 \\
0.74 \\
0.75 \\
0.69\end{array}$ & $\begin{array}{r}0.00 \\
+0.01 \\
+0.07 \\
+0.06 \\
0.00\end{array}$ & $\begin{array}{l}\text { C. } \\
\text { C. } \\
\text { C. } \\
\text { C. } \\
\text { C. }\end{array}$ & $\begin{array}{rrr}+10 & 51 & 12.8 \\
+10 & 49 & 56.9 \\
+10 & 44 & 43.6 \\
+10 & 14 & 38.9 \\
+10 & 13 & 1.8\end{array}$ & $\begin{array}{l}+0.9 \\
+0.2 \\
+1.0 \\
+1.2 \\
+0.5\end{array}$ & $\begin{array}{l}\cdots \\
\cdots \\
\cdots \\
\cdots \\
\cdots\end{array}$ & $\begin{array}{l}\cdots \\
\cdots \\
\cdots \\
\cdots\end{array}$ \\
\hline $\begin{array}{l}20.5 \\
22.5 \\
23.5 \\
24.5 \\
25.5\end{array}$ & $\begin{array}{l}\text { M. } \\
\text { L. } \\
\text { M. } \\
\text { P. } \\
\text { L. }\end{array}$ & & $:$ & $\begin{array}{rrr}2 & 9 & 14.03 \\
2 & 8 & 37.55 \\
2 & 8 & 19.23 \\
2 & 8 & 1.00 \\
2 & 7 & 42.53\end{array}$ & $\begin{array}{l}+0.06 \\
+0.04 \\
+0.03 \\
+0.16 \\
+0.08\end{array}$ & $\begin{array}{r}0.70 \\
.0 .74 \\
0.71 \\
0.74 \\
0.75\end{array}$ & $\begin{array}{l}+0.01 \\
+0.05 \\
+0.02 \\
+0.05 \\
+0.06\end{array}$ & $\begin{array}{l}\text { c. } \\
\text { c. } \\
\text { c. } \\
\text { c. } \\
\text { C. }\end{array}$ & $\begin{array}{rrr}+10 & 8 & 11.6 \\
+10 & 4 & 57.7 \\
+10 & 3 & 21.3 \\
+10 & 1 & 43.6 \\
+10 & 0 & 6.9\end{array}$ & $\begin{array}{l}+0.6 \\
+0.7 \\
+1.3 \\
+0.5 \\
+0.6\end{array}$ & $\begin{array}{l}\cdots \\
\cdots \\
\cdots \\
\cdots\end{array}$ & $\begin{array}{l}\cdots \\
\cdots \\
\cdots \\
\cdots \\
\cdots\end{array}$ \\
\hline $\begin{array}{r}28.5 \\
\text { Nov. } 11.4 \\
17.4 \\
19.4 \\
20.4\end{array}$ & $\begin{array}{l}\text { P. } \\
\text { P. } \\
\text { M. } \\
\text { P. } \\
\text { L. }\end{array}$ & E. & $\dot{.}$ & $\begin{array}{lll}2 & 6 & 47.38 \\
2 & 2 & 35.63 \\
2 & 0 & 55.54 \\
2 & 0 & 23.63 \\
2 & 0 & 8.04\end{array}$ & $\begin{array}{l}+0.15 \\
+0.09 \\
+0.18 \\
+0.10 \\
+0.09\end{array}$ & $\begin{array}{l}0.75 \\
0.73 \\
0.68 \\
0.73 \\
0.70\end{array}$ & $\begin{array}{l}+0.06 \\
+0.05 \\
0.00 \\
+0.05 \\
+0.02\end{array}$ & $\begin{array}{l}\text { C. } \\
\text { C. } \\
\text { C. } \\
\text { c. }\end{array}$ & $\begin{array}{r}+95519.1 \\
+93358.3 \\
+92548.2 \\
+9 \ddot{9} 221.3\end{array}$ & $\begin{array}{l}+1.7 \\
+1.4 \\
+1.1 \\
+1.0\end{array}$ & $\begin{array}{l}\cdots \\
\cdots \\
\cdots \\
\cdots \\
\cdots\end{array}$ & $\begin{array}{l}\cdots \\
\cdots \\
\cdots \\
\cdots\end{array}$ \\
\hline
\end{tabular}




\begin{tabular}{|c|c|c|c|c|c|c|c|c|c|c|c|c|}
\hline \multicolumn{13}{|c|}{ URANUS. } \\
\hline \multicolumn{13}{|c|}{ [No correction for personal equation, see pages A cLxm and A cLXv, nor for equinox, see page A curv, has been applied.] } \\
\hline Date. & $\begin{array}{l}\dot{0} \\
\stackrel{0}{0} \\
\text { 足 } \\
00\end{array}$ & 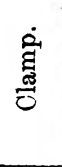 & 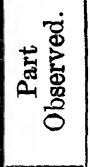 & $\begin{array}{c}\text { Apparent Right } \\
\text { Ascension of } \\
\text { Center. } \\
\end{array}$ & $\begin{array}{c}\text { Correc- } \\
\text { tion to } \\
\text { Am. Eph. }\end{array}$ & $\begin{array}{l}\text { Sidereal } \\
\text { Time of } \\
\text { Transit } \\
\text { of Semi- } \\
\text { diameter. }\end{array}$ & $\begin{array}{c}\text { Correc- } \\
\text { tion to } \\
\text { Am. Eph. }\end{array}$ & 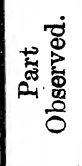 & $\begin{array}{c}\text { Apparent } \\
\text { Declination } \\
\text { of Center. }\end{array}$ & $\begin{array}{c}\text { Correc- } \\
\text { tion to } \\
\text { Am. Eph. }\end{array}$ & $\begin{array}{c}\text { Vertical } \\
\text { Semi- } \\
\text { diameter. }\end{array}$ & $\begin{array}{l}\text { Correc- } \\
\text { tion to } \\
\text { Am. Eph. }\end{array}$ \\
\hline \begin{tabular}{r}
\multicolumn{2}{c}{1904} \\
June 3.5 \\
8.5 \\
12.5 \\
17.5 \\
22.5
\end{tabular} & $\begin{array}{l}\text { Br. } \\
\text { Ei.-Y. } \\
\text { R. } \\
\text { Ei.-Y. } \\
\text { Ei.-Y. }\end{array}$ & W. & $\begin{array}{l}\text { C. } \\
\text { C. } \\
\text { C. } \\
\text { C. } \\
\text { C. }\end{array}$ & $\begin{array}{rcc}\text { h } & \text { m } & \text { s } \\
17 & 53 & 49.36 \\
17 & 52 & 57.99 \\
17 & 52 & 15.96 \\
17 & 51 & 22.92 \\
17 & 50 & 29.67\end{array}$ & $\begin{array}{c}\mathrm{s} \\
+0.13 \\
+0.16 \\
+0.09 \\
+0.14 \\
+0.12\end{array}$ & $\begin{array}{l}\text { s } \\
\cdots \\
\cdots \\
\cdots \\
\cdots \\
\cdots\end{array}$ & $\begin{array}{l}\text { s } \\
\cdots \\
\cdots \\
\cdots \\
\cdots \\
\cdots\end{array}$ & $\begin{array}{l}\text { C. } \\
\text { C. } \\
\text { C. } \\
\text { C. } \\
\text { C. }\end{array}$ & $\begin{array}{rrr}\circ & \prime & \prime \prime \\
-23 & 38 & 28.3 \\
-23 & 38 & 23.5 \\
-23 & 38 & 18.5 \\
-23 & 38 & 11.8 \\
-23 & 38 & 2.8\end{array}$ & $\begin{array}{l}11 \\
+0.3 \\
+0.6 \\
+0.8 \\
+0.3 \\
+0.7\end{array}$ & $\begin{array}{l}\prime \prime \\
\cdots \\
\cdots \\
\cdots \\
\cdots \\
\cdots\end{array}$ & $\begin{array}{l}\prime \\
\cdots \\
\cdots \\
\cdots \\
\cdots\end{array}$ \\
\hline $\begin{array}{ll} & 24.5 \\
\text { July } & 26.4 \\
& 27.4 \\
& 29.4 \\
& 30.4\end{array}$ & $\begin{array}{l}\text { M. } \\
\text { Br. } \\
\text { Ei.-Y. } \\
\text { Ei.-Y. } \\
\text { M. }\end{array}$ & & $\begin{array}{l}\text { C. } \\
\text { C. } \\
\text { C. } \\
\text { C. } \\
\text { C. }\end{array}$ & $\begin{array}{rrr}17 & 50 & 8.36 \\
17 & 45 & 4.59 \\
17 & 44 & 56.89 \\
17 & 44 & 42.10 \\
17 & 44 & 34.80\end{array}$ & $\begin{array}{l}+0.03 \\
+0.11 \\
+0.07 \\
+0.14 \\
+0.04\end{array}$ & $\begin{array}{l}\cdots \\
\cdots \\
\cdots \\
\cdots \\
\cdots\end{array}$ & $\begin{array}{l}\cdots \\
\cdots \\
\cdots \\
\cdots \\
\cdots\end{array}$ & $\begin{array}{l}\text { C. } \\
\text { C. } \\
\text { C. } \\
\text { C. } \\
\text { C. }\end{array}$ & $\begin{array}{lll}-23 & 37 & 59.6 \\
-23 & 36 & 40.3 \\
-23 & 36 & 37.5 \\
-23 & 36 & 32.8 \\
-23 & 36 & 30.5\end{array}$ & $\begin{array}{r}0.0 \\
+0.5 \\
+0.8 \\
+0.5 \\
+0.3\end{array}$ & $\begin{array}{l}\cdots \\
\cdots \\
\cdots \\
\cdots \\
\cdots \\
\cdots\end{array}$ & $\begin{array}{l}\cdots \\
\cdots \\
\cdots \\
\cdots\end{array}$ \\
\hline 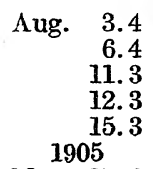 & $\begin{array}{l}\mathrm{Ei}-\mathrm{Y} . \\
\mathrm{Ei}-\mathrm{Y} . \\
\mathrm{M} . \\
\mathrm{Br} . \\
\mathrm{Br} .\end{array}$ & W. & $\begin{array}{l}\text { C. } \\
\text { C. } \\
\text { C. } \\
\text { C. } \\
\text { C. }\end{array}$ & $\begin{array}{llr}17 & 44 & 7.70 \\
17 & 43 & 49.05 \\
17 & 43 & 21.66 \\
17 & 43 & 16.65 \\
17 & 43 & 3.14\end{array}$ & $\begin{array}{l}+0.11 \\
+0.08 \\
+0.11 \\
+0.02 \\
+0.08\end{array}$ & $\begin{array}{l}\cdots \\
\cdots \\
\cdots \\
\cdots \\
\cdots\end{array}$ & $\begin{array}{l}\cdots \\
\cdots \\
\cdots \\
\cdots \\
\cdots\end{array}$ & $\begin{array}{l}\text { C. } \\
\text { C. } \\
\text { C. } \\
\text { C. } \\
\text { C. }\end{array}$ & $\begin{array}{rrr}-23 & 36 & 21.1 \\
-23 & 36 & 13.5 \\
-23 & 36 & 2.6 \\
-23 & 36 & 1.5 \\
-23 & 35 & 56.2\end{array}$ & $\begin{array}{r}0.0 \\
+0.8 \\
+1.1 \\
+0.3 \\
+0.2\end{array}$ & $\begin{array}{l}\cdots \\
\cdots \\
\cdots \\
\cdots \\
\cdots\end{array}$ & $\begin{array}{l}\cdots \\
\cdots \\
\cdots \\
\cdots\end{array}$ \\
\hline $\begin{array}{rr}\text { May } & 27.6 \\
\text { June } & 2.6 \\
3.6 \\
9.5 \\
\text { Aug. } 17.3\end{array}$ & $\begin{array}{l}\text { M. } \\
\text { M. } \\
\text { Ei.-Y. } \\
\text { Hi. } \\
\text { M. }\end{array}$ & $\begin{array}{l}\text { E. } \\
\text { E. } \\
\text { W. }\end{array}$ & $\begin{array}{l}\text { C. } \\
\text { C. } \\
\text { C. } \\
\text { C. } \\
\text { C. }\end{array}$ & $\begin{array}{rrr}18 & 14 & 24.60 \\
18 & 13 & 28.52 \\
18 & 13 & 18.78 \\
18 & 12 & 19.01 \\
18 & 1 & 59.19\end{array}$ & $\begin{array}{l}+0.11 \\
+0.15 \\
+0.11 \\
+0.18 \\
+0.15\end{array}$ & $\begin{array}{l}\cdots \\
\cdots \\
\cdots \\
\cdots \\
\cdots\end{array}$ & $\begin{array}{l}\cdots \\
\cdots \\
\cdots \\
\cdots \\
\cdots\end{array}$ & $\begin{array}{l}\text { C. } \\
\text { C. } \\
\text { C. } \\
\text { C. } \\
\text { C. }\end{array}$ & $\begin{array}{lll}-23 & 39 & 47.6 \\
-23 & 40 & 11.4 \\
-23 & 40 & 15.8 \\
-23 & 40 & 39.1 \\
-23 & 42 & 44.7\end{array}$ & $\begin{array}{r}+0.4 \\
+0.6 \\
+0.2 \\
0.0 \\
+0.1\end{array}$ & $\begin{array}{l}\cdots \\
\cdots \\
\cdots \\
\cdots \\
\cdots\end{array}$ & $\begin{array}{l}\cdots \\
\cdots \\
\cdots \\
\cdots\end{array}$ \\
\hline $\begin{array}{r}18.3 \\
19.3 \\
1906\end{array}$ & $\begin{array}{l}\text { Br. } \\
\text { Hl. }\end{array}$ & & $\begin{array}{l}\text { C. } \\
\text { C. }\end{array}$ & $\begin{array}{lll}18 & 1 & 54.50 \\
18 & 1 & 50.02\end{array}$ & $\begin{array}{l}+0.12 \\
+0.12\end{array}$ & $\cdots$ & $\cdots$ & $\begin{array}{l}\text { C. } \\
\text { C. }\end{array}$ & $\begin{array}{l}-234243.9 \\
-234243.1\end{array}$ & $\begin{array}{l}+1.0 \\
+1.9\end{array}$ & $\begin{array}{l}\cdots \\
\cdots\end{array}$ & $\cdots$ \\
\hline $\begin{array}{r}\text { June } 11.6 \\
14.5\end{array}$ & $\begin{array}{l}\text { Ei.-Y. } \\
\text { Br. }\end{array}$ & & $\begin{array}{l}\text { C. } \\
\text { C. }\end{array}$ & $\begin{array}{lll}18 & 31 & 19.67 \\
18 & 30 & 49.50\end{array}$ & $\begin{array}{l}+0.11 \\
+0.12\end{array}$ & $\begin{array}{l}\cdots \\
\cdots\end{array}$ & 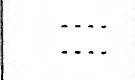 & $\begin{array}{l}\text { C. } \\
\text { C. }\end{array}$ & $\begin{array}{rrr}-23 & 34 & 7.8 \\
-23 & 34 & 32.8\end{array}$ & $\begin{array}{l}+0.4 \\
+0.3\end{array}$ & $\cdots$ & $\cdots$ \\
\hline $\begin{array}{lr} & 22.5 \\
& 25.5 \\
& 29.5 \\
\text { July } & 7.5 \\
\text { Aug. } & 15.4\end{array}$ & $\begin{array}{l}\text { Ei.-Y. } \\
\text { Ei.-Y. } \\
\text { Ei.-Y. } \\
\text { Ei.-Y. } \\
\text { Ei.-Y. }\end{array}$ & & $\begin{array}{l}\text { C. } \\
\text { C. } \\
\text { C. } \\
\text { C. }\end{array}$ & $\begin{array}{lll}18 & 29 & 26.66 \\
18 & 28 & 55.06 \\
18 & 28 & 12.71 \\
18 & 26 & 48.55 \\
18 & 21 & 11.14\end{array}$ & $\begin{array}{l}+0.09 \\
+0.11 \\
+0.06 \\
+0.01 \\
+0.09\end{array}$ & $\begin{array}{l}\cdots \\
\cdots \\
\cdots \\
\cdots \\
\cdots\end{array}$ & $\begin{array}{l}\cdots \\
\cdots \\
\cdots \\
\cdots \\
\cdots\end{array}$ & $\begin{array}{l}\text { C. } \\
\text { C. } \\
\text { C. } \\
\text { C. } \\
\text { C. }\end{array}$ & $\begin{array}{rrr}-23 & 35 & 38.2 \\
-23 & 36 & 2.2 \\
-23 & 36 & 33.5 \\
-23 & 37 & 31.8 \\
-23 & 40 & 47.0\end{array}$ & $\begin{array}{l}+0.1 \\
+0.1 \\
-0.3 \\
+0.1 \\
+0.7\end{array}$ & $\begin{array}{l}\cdots \\
\cdots \\
\cdots \\
\cdots \\
\cdots\end{array}$ & $\begin{array}{l}\cdots \\
\cdots \\
\cdots \\
\cdots\end{array}$ \\
\hline 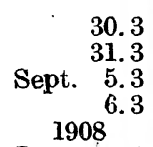 & $\begin{array}{l}\text { Ei.-Y. } \\
\text { Ei.-Y. } \\
\text { P. } \\
\text { Ei.-Y. }\end{array}$ & & $\begin{array}{l}\text { C. } \\
\text { C. } \\
\text { C. }\end{array}$ & $\begin{array}{rrr}18 & 20 & 2.56 \\
18 & 19 & 59.62 \\
18 & 19 & 47.87 \\
18 & 19 & 46.12\end{array}$ & $\begin{array}{l}+0.07 \\
+0.11 \\
+0.14 \\
+0.11\end{array}$ & $\begin{array}{l}\cdots \\
\cdots \\
\cdots \\
\cdots\end{array}$ & $\begin{array}{l}\cdots \\
\cdots \\
\cdots \\
\cdots\end{array}$ & $\begin{array}{l}\text { C. } \\
\text { C. } \\
\text { C. } \\
\text { C. }\end{array}$ & $\begin{array}{lll}-23 & 41 & 16.7 \\
-23 & 41 & 17.2 \\
-23 & 41 & 20.9 \\
-23 & 41 & 21.8\end{array}$ & $\begin{array}{l}+0.2 \\
+0.8 \\
+0.9 \\
+0.4\end{array}$ & $\begin{array}{l}\cdots \\
\cdots \\
\cdots \\
\cdots\end{array}$ & $\begin{array}{l}\cdots \\
\cdots \\
\cdots\end{array}$ \\
\hline $\begin{array}{r}\text { June } 13.6 \\
15.6 \\
16.6 \\
18.6 \\
22.5\end{array}$ & $\begin{array}{l}\text { P. } \\
\text { P. } \\
\text { Fk. } \\
\text { Fk. }\end{array}$ & & $\begin{array}{l}\text { C. } \\
\text { C. } \\
\text { C. } \\
\text { C. }\end{array}$ & $\begin{array}{rrr}19 & 8 & 53.57 \\
19 & 8 & 34.90 \\
19 & 8 & 25.28 \\
19 & 8 & 6.12 \\
19 & 7 & 26.72\end{array}$ & $\begin{array}{l}+0.24 \\
+0.29 \\
+0.16 \\
+0.23 \\
+0.19\end{array}$ & $\begin{array}{l}\cdots \\
\cdots \\
\cdots \\
\cdots \\
\cdots\end{array}$ & $\begin{array}{l}\cdots \\
\cdots \\
\cdots \\
\cdots \\
\cdots\end{array}$ & $\begin{array}{l}\text { C. } \\
\text { C. } \\
\text { C. } \\
\text { c. } \\
\text { c. }\end{array}$ & $\begin{array}{lll}-22 & 55 & 40.4 \\
-22 & 56 & 12.7 \\
-22 & 56 & 29.0 \\
-22 & 57 & 2.7 \\
-22 & 58 & 9.6\end{array}$ & $\begin{array}{l}+0.5 \\
+0.8 \\
+1.1 \\
+0.7 \\
+0.8\end{array}$ & $\begin{array}{l}\cdots \\
\cdots \\
\cdots \\
\cdots \\
\cdots\end{array}$ & $\begin{array}{l}\cdots \\
\cdots \\
\cdots \\
\cdots\end{array}$ \\
\hline 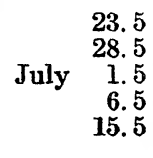 & $\begin{array}{l}\text { M. } \\
\text { M. } \\
\text { Fk. } \\
\text { P. } \\
\text { Fk. }\end{array}$ & & $\begin{array}{l}\text { c. } \\
\text { c. } \\
\text { c. } \\
\text { c. }\end{array}$ & $\begin{array}{rrr}19 & 7 & 16.72 \\
19 & 6 & 25.90 \\
19 & 5 & 54.83 \\
19 & 5 & 2.82 \\
19 & 3 & 29.50\end{array}$ & $\begin{array}{l}+0.20 \\
+0.22 \\
+0.13 \\
+0.15 \\
+0.22\end{array}$ & $\begin{array}{l}\cdots \\
\cdots \cdots \\
\cdots \\
\cdots \\
\cdots\end{array}$ & $\begin{array}{l}\cdots \\
\cdots \\
\cdots \\
\cdots \\
\cdots\end{array}$ & $\begin{array}{l}\text { c. } \\
\text { c. } \\
\text { c. } \\
\text { c. }\end{array}$ & $\begin{array}{rrr}-22 & 58 & 26.6 \\
-22 & 59 & 51.8 \\
-23 & 0 & 42.0 \\
-23 & 2 & 5.6 \\
-23 & 4 & 32.3\end{array}$ & $\begin{array}{l}+0.6 \\
+0.1 \\
+0.6 \\
+1.0 \\
+0.9\end{array}$ & $\begin{array}{l}\cdots \\
\cdots \\
\cdots \\
\cdots \\
\cdots\end{array}$ & $\begin{array}{l}\cdots \\
\cdots \\
\cdots \\
\cdots\end{array}$ \\
\hline $\begin{array}{r}16.5 \\
20.5 \\
28.4 \\
29.4 \\
\text { Aug. } \quad 1.4\end{array}$ & $\begin{array}{l}\text { M. } \\
\text { M. } \\
\text { Fk. } \\
\text { P. } \\
\text { Fk. }\end{array}$ & & $\begin{array}{l}\text { C. } \\
\text { C. } \\
\text { C. } \\
\text { c. }\end{array}$ & $\begin{array}{lll}19 & 3 & 19.19 \\
19 & 2 & 38.59 \\
19 & 1 & 20.33 \\
19 & 1 & 10.97 \\
19 & 0 & 43.25\end{array}$ & $\begin{array}{l}+0.17 \\
+0.15 \\
+0.12 \\
+0.17 \\
+0.12\end{array}$ & $\begin{array}{l}\cdots \\
\cdots \\
\cdots \\
\cdots\end{array}$ & $\begin{array}{l}\ldots, \\
\cdots \\
\cdots \\
\cdots \\
\cdots\end{array}$ & $\begin{array}{l}\text { C. } \\
\text { C. } \\
\text { C. } \\
\text { C. }\end{array}$ & $\begin{array}{lll}-23 & 4 & 48.3 \\
-23 & 5 & 50.6 \\
-23 & 7 & 45.7 \\
-23 & 7 & 59.5 \\
-23 & 8 & 40.0\end{array}$ & $\begin{array}{l}+0.6 \\
+0.1 \\
+1.0 \\
+1.0 \\
+0.6\end{array}$ & $\begin{array}{l}\cdots \\
\cdots \\
\cdots \\
\cdots \\
\cdots\end{array}$ & $\begin{array}{l}\cdots \\
\cdots \\
\cdots \\
\cdots\end{array}$ \\
\hline $\begin{array}{r}3.4 \\
1909\end{array}$ & P. & W. & C. & $\begin{array}{rrr}19 & 0 & 25.36 \\
18 & 59 & 34.38\end{array}$ & $\begin{array}{l}+0.17 \\
+0.15\end{array}$ & $\cdots$ & $\ldots$ & C. & $\begin{array}{rrr}-23 & 9 & 5.6 \\
-23 & 10 & 17.3\end{array}$ & $\begin{array}{l}+0.6 \\
+0.6\end{array}$ & $\begin{array}{l}\cdots \\
\cdots\end{array}$ & $\cdots$ \\
\hline $\begin{array}{r}\text { June } 15.6 \\
16.6\end{array}$ & $\begin{array}{l}\text { M. } \\
\text { L. }\end{array}$ & $\begin{array}{l}\mathbf{E} . \\
\mathbf{E} .\end{array}$ & C. & $\begin{array}{rrr}19 & 27 & 14.00 \\
19 & 27 & 5.19\end{array}$ & $\begin{array}{l}+0.07 \\
+0.23\end{array}$ & $\cdots$ & $\ldots$ & c. & $\begin{array}{l}-222437.4 \\
-222455.8\end{array}$ & $\begin{array}{l}-0.7 \\
+0.6\end{array}$ & $\cdots$ & $\cdots$ \\
\hline
\end{tabular}


RESUltS OF OBSERVATIONS OF THE SUN, MOON, AND PLANETS.

\begin{tabular}{|c|c|c|c|c|c|c|c|c|c|c|c|c|}
\hline \multicolumn{13}{|c|}{ URANUS. } \\
\hline Date. & 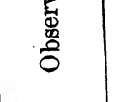 & $\underset{\Xi}{\tilde{\sigma}}$ & 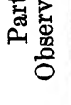 & $\begin{array}{l}\text { Apparent Right } \\
\text { Ascension of } \\
\text { Center. }\end{array}$ & $\begin{array}{c}\text { Correc- } \\
\text { tion to } \\
\text { Am. Eph. }\end{array}$ & $\begin{array}{l}\text { Time of } \\
\text { Transit } \\
\text { of Semi- } \\
\text { diameter. }\end{array}$ & $\begin{array}{c}\text { Correc- } \\
\text { tion to } \\
\text { Am. Eph. }\end{array}$ & 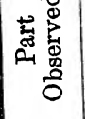 & $\begin{array}{l}\text { Apparent } \\
\text { Declination } \\
\text { of Center. }\end{array}$ & $\begin{array}{c}\text { Correc- } \\
\text { tion to } \\
\text { Am. Eph. }\end{array}$ & $\begin{array}{c}\text { Vertical } \\
\text { Semi- } \\
\text { diameter. }\end{array}$ & $\begin{array}{l}\text { Correc- } \\
\text { tion to } \\
\text { Am. Eph }\end{array}$ \\
\hline $\begin{array}{c}1909 \\
\text { June } 23.6\end{array}$ & $\mathrm{~L}$. & E. & C. & & $\begin{array}{l}\mathrm{s} \\
+0.32\end{array}$ & $\begin{array}{l}\mathrm{s} \\
\ldots\end{array}$ & s. & C. & $\begin{array}{ccc}\circ & \prime \prime & \prime \prime \\
-22 & 27 & 16.5\end{array}$ & $\begin{array}{r}\prime \prime \\
+1.7\end{array}$ & $\begin{array}{l}\prime \prime \\
\ldots .\end{array}$ & $\ldots$ \\
\hline 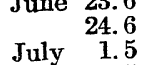 & M. & . & C. & $\begin{array}{l}192059.86 \\
192550.09 \\
1924.40 .59\end{array}$ & & $\cdots$ & $\cdots$ & C. & $\begin{array}{rrr}-22 & 27 & 38.8 \\
-22 & 30 & 5.3\end{array}$ & +0.1 & $\cdots$ & $\cdots$ \\
\hline $\begin{array}{l}\text { July } \begin{array}{l}1.5 \\
2.5\end{array} \\
\quad 3.5\end{array}$ & P. & & c. & $\begin{array}{l}1924.40 .59 \\
192430.48\end{array}$ & $\begin{array}{l}+0.17 \\
+0.19\end{array}$ & $\cdots$ & $\cdots$ & C.: & $\begin{array}{rrr}-22 & 30 & 5.3 \\
-22 & 30 & 26.1 \\
\end{array}$ & $\begin{array}{l}+0.2 \\
+0.5\end{array}$ & $\begin{array}{l}\cdots \\
\cdots\end{array}$ & $\begin{array}{l}\cdots \\
\cdots\end{array}$ \\
\hline 3.5 & $\mathrm{~L}$. & & C. & 192420.34 & +0.21 & $\cdots$ & $\cdots \cdot$ & C. & $\begin{array}{llll}-22 & 30 & 47.0\end{array}$ & +0.7 & $\cdots$ & $\cdots$ \\
\hline $\begin{array}{r}4.5 \\
27.5\end{array}$ & M. & & $\dot{\mathrm{c}}$. & 192015.61 & +0.19 & $\cdots$ & $\cdots$ & C. & $\begin{array}{l}-2231 \\
-2238 \\
-24.6\end{array}$ & $\begin{array}{l}+0.2 \\
+1.0\end{array}$ & $\cdots$ & $\cdots$ \\
\hline $\begin{array}{r}28.5 \\
30.4\end{array}$ & M. & & $\begin{array}{l}\text { C. } \\
\text { C. }\end{array}$ & $\begin{array}{rrr}19 & 20 & 5.77 \\
19 & 19 & 46.48\end{array}$ & $\begin{array}{l}+0.11 \\
+0.15\end{array}$ & $\cdots$ & $\cdots$ & C. & $\begin{array}{l}-223914.3 \\
-223950.5\end{array}$ & $\begin{array}{l}-0.1 \\
+0.3\end{array}$ & $\ldots$ & $\cdots$ \\
\hline Aug. $\quad 1.4$ & $\mathbf{P}$ & & $\cdot$ & $\cdots: \cdots \cdots$ & +0.15 & $\cdots$ & $\cdots$ & C. & -224026.4 & $\begin{array}{l}+0.0 \\
+0.3\end{array}$ & $\cdots$ & $\cdots$ \\
\hline $\begin{array}{l}2.4 \\
4.4\end{array}$ & L. & & C. & $\begin{array}{lll}19 & 19 & 18.14 \\
19 & 18 & 59.66\end{array}$ & +0.19 & $\cdots$ & $\cdots$ & C. & -224042.5 & +1.9 & $\cdots$ & $\ldots$ \\
\hline $\begin{array}{r}4.4 \\
6.4\end{array}$ & L. & & C. & $\begin{array}{l}1918 \quad 59.66 \\
19 \quad 18 \quad 41.53\end{array}$ & $\begin{array}{l}+0.21 \\
+0.16\end{array}$ & $\cdots$ & $\begin{array}{l}\cdots \\
\cdots\end{array}$ & C. & $\begin{array}{l}-224117.7 \\
-2241 \quad 51.7\end{array}$ & $\begin{array}{l}+1.1 \\
+0.8\end{array}$ & $\cdots$ & $\begin{array}{l}\cdots \\
\cdots\end{array}$ \\
\hline $\begin{array}{l}1910 \\
\text { June } 21.6\end{array}$ & P. & & C. & $1944 \quad 43.75$ & +0.33 & $\cdots$ & $\cdots$ & C. & -214737.8 & +1.3 & $\cdots$ & \\
\hline 22.6 & M. & & c. & 194434.62 & +0.19 & $\cdots$ & $\cdots$ & C. & $\begin{array}{lll}-21 & 48 & 1.1\end{array}$ & +1.5 & $\cdots$ & $\cdots$ \\
\hline $\begin{array}{l}23.6 \\
24.6\end{array}$ & L. & & C. & 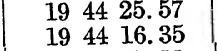 & $\begin{array}{l}+0.23 \\
+0.19\end{array}$ & $\cdots$ & $\cdots \cdot$ & C. & $\begin{array}{l}-214824.3 \\
-214848.9\end{array}$ & $\begin{array}{l}+2.0 \\
+1.3\end{array}$ & $\cdots$ & $\cdots$ \\
\hline 25.6 & $\mathrm{~L}$ & & C. & 1944.7 .11 & +0.23 & $\cdots$ & $\cdots$ & C. & -214911.8 & +2.4 & $\cdots$ & $\cdots$ \\
\hline 26.5 & M. & & C. & 194357.83 & +0.31 & $\cdots$ & .... & C. & -214936.9 & +1.4 & $\cdots$ & $\cdots$ \\
\hline July $\begin{array}{l}19.5 \\
20.5\end{array}$ & L. & & C. & 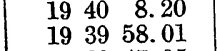 & $\begin{array}{l}+0.16 \\
+0.15\end{array}$ & $\cdots$ & $\cdots$ & C. & $\begin{array}{lll}-21 & 59 & 7.7 \\
-21 & 59 & 32.8\end{array}$ & $\begin{array}{l}+1.8 \\
+1.2\end{array}$ & $\cdots$ & $\cdots$ \\
\hline $\begin{array}{l}21.5 \\
22.5\end{array}$ & P. & & C. & $\begin{array}{lll}19 & 39 & 47.95 \\
19 & 39 & 37.75\end{array}$ & $\begin{array}{l}+0.26 \\
+0.20\end{array}$ & $\begin{array}{l}\cdots \\
\cdots\end{array}$ & $\ldots$ & C. & $\begin{array}{l}-215956.3 \\
-22.022 .4\end{array}$ & $\begin{array}{l}+2.2 \\
+0.4\end{array}$ & $\cdots$ & $\cdots$ \\
\hline 23.5 & $\mathbf{P}$ & & c. & 193927.67 & +0.24 & $\cdots$ & $\cdots$ & $\mathrm{C}$. & $-22 \quad 0 \quad 46.7$ & +0.2 & $\cdots$ & $\cdots$ \\
\hline $\begin{array}{r}24.5 \\
44\end{array}$ & M. & & C. & $\begin{array}{lll}19 & 39 & 17.56\end{array}$ & +0.22 & $\cdots$ & $\cdots$ & C. & $\begin{array}{lll}-22 & 1 & 10.8 \\
-22 & 5 & 21.9\end{array}$ & +0.1 & $\cdots$ & $\cdots$ \\
\hline $\begin{array}{ll}\text { Aug. } & 4.4 \\
5.4\end{array}$ & $\begin{array}{l}\mathrm{P} . \\
\mathrm{M} .\end{array}$ & & C. & $\begin{array}{lll}19 & 37 & 29.80 \\
19 & 37 & 20.33\end{array}$ & $\begin{array}{l}+0.35 \\
+0.30\end{array}$ & $\cdots$ & $\ldots$. & $\begin{array}{l}\text { C. } \\
\text { C. }\end{array}$ & 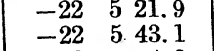 & $\begin{array}{l}+1.1 \\
+1.5\end{array}$ & $\cdots$ & $\cdots$ \\
\hline 6.4 & $\mathrm{~L}$. & E. & C. & $1937 \quad 10.97^{\prime}$ & +0.27 & $\cdots$ & $\cdots$ & C. & $\begin{array}{lll}-22 & 6 & 4.8\end{array}$ & +1.2 & $\cdots$ & $\cdots$ \\
\hline \multicolumn{13}{|c|}{ NEPTUNE. } \\
\hline \multicolumn{13}{|c|}{ [No correction for personal equation, see pages A cLxir and A cLxv nor for equinox, see page A cLrv, has been applied.] } \\
\hline 1903 & & & & & & & & & & & & \\
\hline Oct. $\begin{array}{l}12.7 \\
13.7\end{array}$ & $\begin{array}{l}\text { Br. } \\
\text { R. }\end{array}$ & W. & C. & 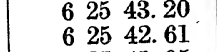 & $\begin{array}{l}+0.04 \\
-0.01\end{array}$ & $\cdots \cdot$ & $\cdots$ & G. & $\begin{array}{r}+221440.0 \\
+221444.8\end{array}$ & -0.6 & $\cdots$ & $\cdots$ \\
\hline 14.7 & & & C. & 62541.95 & +0.02 & $\cdots$ & $\cdots$ & C. & $\begin{array}{r}+221444.4 \\
\end{array}$ & +0.1 & $\cdots$ & $\cdots$ \\
\hline $\begin{array}{ll}\text { Nov. } & 3.6 \\
& 6.6\end{array}$ & $\left|\begin{array}{|l}\text { Ei. }-\mathrm{Y} . \\
\text { Ei.-Y. }\end{array}\right|$ & & C. & $\begin{array}{lll}6 & 24 & 58.69 \\
6 & 24 & 47.52\end{array}$ & $\begin{array}{r}0.00 \\
-0.09\end{array}$ & $\cdots$ & $\cdots \cdot$ & c. & $\begin{array}{l}+221440.2 \\
+221443.6\end{array}$ & $\begin{array}{l}-0.6 \\
+0.1\end{array}$ & $\cdots$ & $\cdots$ \\
\hline 9.6 & Ei.-Y. & & & $\begin{array}{lll}6 & 24 & 35.32\end{array}$ & -0.11 & $\ldots$ & $\cdots$ & C. & +221446.8 & -0.3 & $\cdots$ & $\cdots$ \\
\hline Dec. 11.5 & $\mathrm{Ei}-\mathrm{Y}$ & & c. & $\begin{array}{lll}6 & 21 & 29.52\end{array}$ & -0.03 & $\cdots \cdots$ & $\cdots$ & C. & $\begin{array}{r}+22168.9 \\
+221650.2\end{array}$ & +0.1 & $\cdots$ & $\cdots$ \\
\hline 1904 & Ei.-Y. & & C. & 6. 2010.64 & -0.03 & $\cdots$ & & & & +0. & $\cdots$ & $\cdots$ \\
\hline Jan. 25.4 & Ei.-Y. & & C. & $\begin{array}{lll}6 & 16 & 14.19\end{array}$ & -0.06 & $\cdots \cdot$ & $\cdots$ & C. & +221910.7 & 0.0 & $\cdots$ & $\cdots$ \\
\hline $\begin{array}{l}27.4 \\
30.4\end{array}$ & $\mid \begin{array}{l}\text { Ei.-Y. } \\
\text { Ei. }-Y .\end{array}$ & & C. & $\begin{array}{rrr}6 & 16 & 2.27 \\
6 & 15 & 45.05\end{array}$ & $\begin{array}{r}-0.04 \\
0.00\end{array}$ & - $\ldots$. & $\cdots \cdot$ & c. & $\begin{array}{r}+221919.7 \\
+221931.2\end{array}$ & $\begin{array}{r}+0.8 \\
0.0\end{array}$ & $\cdots$ & $\cdots$ \\
\hline Feb. 23.3 & Ei.-R. & & u. & $\begin{array}{lll}6 & 14 & 2.13\end{array}$ & -0.04 & $\cdots$ & $\cdots$ & c. & $\begin{array}{r}+2221 \quad 1.9 \\
+22\end{array}$ & $\begin{array}{r}0.0 \\
+1.1\end{array}$ & $\cdots \cdot$ & $\cdots$ \\
\hline $\begin{array}{l}24.3 \\
25.3\end{array}$ & $\mid \begin{array}{l}\mathrm{Ei} .-\mathrm{M} . \\
\mathrm{Ei} .-\mathrm{R} .\end{array}$ & & $\begin{array}{l}\text { C. } \\
\text { C. }\end{array}$ & $\begin{array}{llll}6 & 13 & 59.39 \\
6 & 13 & 56.76\end{array}$ & $\begin{array}{l}-0.06 \\
-0.10\end{array}$ & $\cdots$ & $\cdots$ & C. & 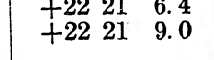 & $\begin{array}{l}+1.1 \\
+0.3\end{array}$ & $\cdots$ & $\cdots$ \\
\hline & Ei.-M. & W. & C. & 61352.01 & -0.07 & $\cdots$ & $\cdots$ & C. & +222115.9 & +0.4 & $\cdots \cdot$ & ... \\
\hline Nov. 24.6 & Br. & $\mathbf{E}$ & C. & $\begin{array}{rrr}6 & 33 & 6.41 \\
6 & 30 & 14.80\end{array}$ & -0.04 & $\cdots$ & $\cdots$ & $\begin{array}{l}\text { C. } \\
\text { C. }\end{array}$ & $\begin{array}{r}+22127.9 \\
+221412.7\end{array}$ & 0.0 & $\cdots$ & $\cdots$ \\
\hline $\begin{aligned} \text { Dec. } 20.5 \\
21.5\end{aligned}$ & $\begin{array}{l}\text { Br. } \\
\text { Ei.-M. }\end{array}$ & E. & C. & $\begin{array}{rrr}6 & 30 & 14.80 \\
6 & 30 & 7.56\end{array}$ & $\begin{array}{l}0.00 \\
0.00\end{array}$ & $\cdots$ & $\cdots$ & c. & $\begin{array}{r}+221412.7 \\
+221419.5\end{array}$ & $\begin{array}{l}-0.3 \\
+1.0\end{array}$ & $\cdots \cdot$ & $\cdots$ \\
\hline & & & & & & & & & & & & \\
\hline
\end{tabular}




\begin{tabular}{|c|c|c|c|c|c|c|c|c|c|c|c|c|}
\hline \multicolumn{13}{|c|}{ NEPTUNE. } \\
\hline \multicolumn{13}{|c|}{ [No correction for personal equation, see pages A crxum and A cuxv, nor for equinox, see page A culv, has been applied.] } \\
\hline Date. & $\begin{array}{l}\text { 离 } \\
\text { 总 } \\
0 \\
0\end{array}$ & 窎 & 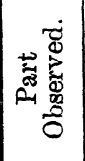 & $\begin{array}{l}\text { Apparent Right } \\
\text { Ascension of } \\
\text { Center. }\end{array}$ & $\begin{array}{c}\text { Correc- } \\
\text { tion to } \\
\text { Am. Eph. }\end{array}$ & $\mid \begin{array}{c}\text { Sidereal } \\
\text { Time of } \\
\text { Transit } \\
\text { of Semi- } \\
\text { diameter. }\end{array}$ & $\begin{array}{c}\text { Correc- } \\
\text { tion to } \\
\text { Am. Eph. }\end{array}$ & 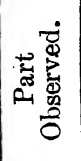 & $\begin{array}{l}\text { Apparent } \\
\text { Declination } \\
\text { of Center. }\end{array}$ & $\mid \begin{array}{c}\text { Correc- } \\
\text { tion to } \\
\text { Am. Eph. }\end{array}$ & $\begin{array}{c}\text { Vertical } \\
\text { Semi- } \\
\text { diameter. }\end{array}$ & $\begin{array}{c}\text { Correc- } \\
\text { tion to } \\
\text { Am. Eph. }\end{array}$ \\
\hline \begin{tabular}{rr}
\multicolumn{1}{c}{1905} \\
Jan. 16.4 \\
18.4 \\
19.4 \\
20.4 \\
Feb. 13.4
\end{tabular} & $\begin{array}{l}\text { Ei.-Y. } \\
\text { Ei.-M. } \\
\text { Y. } \\
\text { Br. } \\
\text { Y. }\end{array}$ & E. & $\begin{array}{l}\text { c. } \\
\text { c. } \\
\text { c. } \\
\text { c. } \\
\text { c. }\end{array}$ & $\begin{array}{lcc}\mathrm{h} & \mathrm{m} & \dot{\mathrm{s}} \\
6 & 27 & 0.89 \\
6 & 26 & 47.45 \\
6 & 26 & 47.45 \\
6 & 26 & 40.83 \\
6 & 26 & 34.25 \\
6 & 24 & 22.34\end{array}$ & $\begin{array}{c}s \\
-0.04 \\
-0.06 \\
-0.05 \\
-0.06 \\
-0.01\end{array}$ & $\begin{array}{l}\mathrm{s} \\
\cdots \\
\cdots \\
\cdots \\
\cdots \\
\cdots\end{array}$ & $\begin{array}{l}\mathrm{s} \\
\cdots \\
\cdots \\
\cdots \\
\cdots \\
\cdots\end{array}$ & $\begin{array}{l}\text { c. } \\
\text { c. } \\
\text { c. } \\
\text { c. }\end{array}$ & 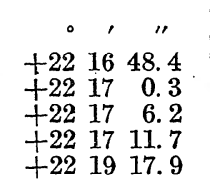 & $\begin{array}{r}1 " \\
-0.1 \\
+0.4 \\
+0.6 \\
+0.4 \\
+0.8\end{array}$ & $\begin{array}{l}\prime \prime \\
\cdots . \\
\cdots \\
\cdots \\
\cdots \\
\cdots\end{array}$ & $\begin{array}{l}\prime \\
\cdots \\
\cdots \\
\cdots \\
\cdots\end{array}$ \\
\hline $\begin{array}{l}14.4 \\
15.4 \\
16.4 \\
17.4 \\
18.4\end{array}$ & $\begin{array}{l}\text { Br. } \\
\text { M. } \\
\text { Y. } \\
\text { Br. } \\
\text { Ei.-M. }\end{array}$ & & $\begin{array}{l}\text { C. } \\
\text { C. } \\
\text { C. } \\
\text { C. } \\
\text { C. }\end{array}$ & $\begin{array}{lrr}6 & 24 & 18.09 \\
6 & 24 & 14.02 \\
6 & 24 & 10.11 \\
6 & 24 & 6.24 \\
6 & 24 & 2.55\end{array}$ & $\begin{array}{l}-0.07 \\
-0.07 \\
-0.03 \\
-0.08 \\
-0.08\end{array}$ & $\begin{array}{l}\cdots \\
\cdots \\
\cdots \\
\cdots \\
\cdots\end{array}$ & $\begin{array}{l}\cdots \\
\cdots \\
\cdots \\
\cdots \\
\cdots\end{array}$ & $\begin{array}{l}\text { c. } \\
\text { c. } \\
\text { c. } \\
\text { c. }\end{array}$ & $\begin{array}{r}+221921.8 \\
+221925.8 \\
+221930.7 \\
+221935.6 \\
+221940.5\end{array}$ & $\begin{array}{r}0.0 \\
-0.6 \\
-0.3 \\
+0.1 \\
+0.6\end{array}$ & $\begin{array}{l}\cdots \\
\cdots \\
\cdots \\
\cdots \\
\cdots\end{array}$ & $\begin{array}{l}\cdots \\
\cdots \\
\cdots \\
\cdots\end{array}$ \\
\hline $\begin{array}{cc} & 24.3 \\
\text { Mar. } & 10.3 \\
\text { Nov. } 14.6 \\
\text { Dec. } 11.6\end{array}$ & $\begin{array}{l}\text { Ei.-Y. } \\
\text { Ei.-Y. } \\
\text { Bs. } \\
\text { Hi. } \\
\text { Br. }\end{array}$ & $\begin{array}{l}\text { E. } \\
\text { W. }\end{array}$ & $\begin{array}{l}\text { c. } \\
\text { c. } \\
\text { c. } \\
\text { c. } \\
\text { c. }\end{array}$ & $\begin{array}{rrr}6 & 23 & 43.08 \\
6 & 23 & 16.64 \\
6 & 43 & 47.63 \\
6 & 43 & 9.20 \\
6 & 41 & 13.97\end{array}$ & $\begin{array}{l}-0.04 \\
-0.09 \\
\sim 0.08 \\
-0.08 \\
-0.02\end{array}$ & $\begin{array}{l}\cdots . . \\
\cdots \cdots \\
\cdots \cdots \\
\cdots \\
\cdots\end{array}$ & $\begin{array}{l}\cdots \\
\cdots \\
\cdots \\
\cdots \\
\cdots\end{array}$ & $\begin{array}{l}\text { C. } \\
\text { C. } \\
\text { C. } \\
\text { C. } \\
\text { C. }\end{array}$ & 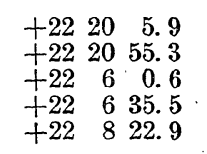 & $\begin{array}{l}+0.6 \\
+0.1 \\
+0.2 \\
+1.7 \\
+0.1\end{array}$ & $\begin{array}{l}\cdots \\
\cdots \\
\cdots \\
\cdots \\
\cdots\end{array}$ & $\begin{array}{l}\cdots \\
\cdots \\
\cdots \\
\cdots\end{array}$ \\
\hline 1906 & Hi. & & $c$. & $641 \quad 0.33$ & -0.05 & $\cdots$ & $\cdots$ & C. & $+22 \quad 837.1$ & +1.0 & $\cdots$ & $\ldots$ \\
\hline $\begin{array}{l}\text { Jan. } \\
\text { Feb. } \\
9.4 \\
16.4\end{array}$ & $\begin{array}{l}\text { Ei.-Y. } \\
\text { Br. } \\
\text { Br. }\end{array}$ & & $\begin{array}{l}\text { C. } \\
\text { C; }\end{array}$ & $\begin{array}{lll}6 & 37 & 46.18 \\
6.34 & 31.76 \\
6 & 33 & 59.67\end{array}$ & $\begin{array}{l}-0.07 \\
-0.06 \\
-0.03\end{array}$ & $\begin{array}{l}\cdots \\
\cdots \\
\cdots\end{array}$ & $\begin{array}{l}\cdots \\
\cdots \\
\cdots\end{array}$ & $\begin{array}{l}\text { c. } \\
\text { c. } \\
\text { c. }\end{array}$ & $\begin{array}{rrr}+22 & 11 & 53.3 \\
+22 & 15 & 28.4 \\
+22 & 16 & 8.7\end{array}$ & $\begin{array}{l}+0.7 \\
+0.5 \\
+0.2\end{array}$ & $\begin{array}{l}\cdots \\
\cdots \\
\cdots\end{array}$ & $\begin{array}{l}\cdots \\
\cdots \\
\cdots\end{array}$ \\
\hline $\begin{array}{r}19.4 \\
20.4 \\
24.3 \\
28.3 \\
\text { Mar. } \quad 2.3\end{array}$ & $\begin{array}{l}\text { Bs. } \\
\text { Br. } \\
\text { Hl. } \\
\text { Bs. } \\
\text { Br. }\end{array}$ & & $\begin{array}{l}\text { c. } \\
\text { c. } \\
\text { c. } \\
\text { c. }\end{array}$ & $\begin{array}{lll}6 & 33 & 47.67 \\
6 & 33 & 43.94 \\
6 & 33 & 30.14 \\
6 & 33 & 18.52 \\
6 & 33 & 13.57\end{array}$ & $\begin{array}{l}-0.03 \\
-0.01 \\
-0.08 \\
-0.07 \\
-0.01\end{array}$ & $\begin{array}{l}\cdots \\
\cdots \\
\cdots \\
\cdots \\
\cdots\end{array}$ & $\begin{array}{l}\cdots \\
\cdots \\
\cdots \\
\cdots \\
\cdots\end{array}$ & $\begin{array}{l}\text { c. } \\
\text { C. } \\
\text { C. } \\
\text { C. }\end{array}$ & $\begin{array}{r}+221635.8 \\
+221630.4 \\
+221651.6 \\
+22179.9 \\
+2217 \quad 18.3\end{array}$ & $\begin{array}{l}+0.6 \\
-0.1 \\
+1.0 \\
+0.8 \\
+0.5\end{array}$ & $\begin{array}{l}\cdots \\
\cdots \\
\cdots \\
\cdots \\
\cdots\end{array}$ & $\begin{array}{l}\cdots \\
\cdots \\
\cdots \\
\cdots\end{array}$ \\
\hline $\begin{array}{r}6.3 \\
10.3 \\
1907\end{array}$ & $\begin{array}{l}\mathrm{Br} . \\
\mathrm{HI}\end{array}$ & W. & c. & $\begin{array}{lll}\begin{array}{rlr}6 & 33 & 5.23 \\
6 & 32 & 59.11\end{array}\end{array}$ & $\begin{array}{l}+0.01 \\
-0.02\end{array}$ & $\cdots$ & $\cdots$ & c. & $\begin{array}{r}+221734.3 \\
+221749.8\end{array}$ & $\begin{array}{l}+0.3 \\
+1.2\end{array}$ & $\begin{array}{ll}\cdots \\
\cdots\end{array}$ & $\cdots$ \\
\hline $\begin{array}{r}\text { Nov. } 13.6 \\
14.6\end{array}$ & Mi. & E. & $\begin{array}{l}\text { c. } \\
\text { c. }\end{array}$ & $\begin{array}{lll}7 & 3 & 25.29 \\
7 & 3 & 21.69\end{array}$ & $\begin{array}{l}-0.13 \\
-0.04\end{array}$ & $\cdots$ & .... & c. & $\begin{array}{r}+214815.3 \\
+2148 \quad 20.7\end{array}$ & $\begin{array}{l}+0.3 \\
+0.9\end{array}$ & $\begin{array}{l}\cdots \\
\cdots\end{array}$ & $\begin{array}{l}\therefore \\
\cdots\end{array}$ \\
\hline $\begin{array}{r}24.6 \\
25.6 \\
\text { Dec. } 19.5\end{array}$ & $\begin{array}{l}\text { M. } \\
\text { P. }\end{array}$ & & $\begin{array}{l}\text { c. } \\
\text { c. }\end{array}$ & $\begin{array}{lrr}7 & 2 & 38.30 \\
7 & 2 & 33.42 \\
7 & 0 & 7.97\end{array}$ & $\begin{array}{l}-0.10 \\
-0.03 \\
-0.08\end{array}$ & & $\begin{array}{l}\cdots \\
\cdots \\
\cdots\end{array}$ & $\begin{array}{l}\text { c. } \\
\text { c. } \\
\text { c. }\end{array}$ & $\begin{array}{r}+214918.2 \\
+214925.7 \\
+215253.7\end{array}$ & $\begin{array}{l}-0.1 \\
+0.5 \\
+0.5\end{array}$ & $\begin{array}{l}\cdots \\
\cdots \\
\cdots\end{array}$ & $\begin{array}{l}\cdots \\
\cdots \\
\cdots\end{array}$ \\
\hline $\begin{array}{r}21.5 \\
1908.5\end{array}$ & $\mathrm{HI}$. & & $\mathrm{c}$ & $\begin{array}{lll}6 & 59 & 54.16 \\
& & \end{array}$ & -0.05 & … & $\cdots$ & c. & $\begin{array}{r}5313.8 \\
+215740.5\end{array}$ & +0.4 & $\cdots$ & $\cdots$ \\
\hline Jan. 15.5 & $\mathrm{Hl}$. & & c. & 65654.62 & -0.03 & $\cdots$ & $\cdots$ & C. & +215740.5 & +0.3 & $\cdots$ & $\cdots$ \\
\hline 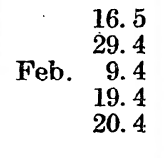 & $\begin{array}{l}\mathbf{P} . \\
\text { P. } \\
\text { Hil. } \\
\mathbf{P} . \\
\text { M. }\end{array}$ & & $\begin{array}{l}\text { C. } \\
\text { C. } \\
\text { C. } \\
\text { C. } \\
\text { C. }\end{array}$ & $\begin{array}{lll}6 & 56 & 47.54 \\
6 & 55 & 19.89 \\
6 & 54 & 14.52 \\
6 & 53 & 25.24 \\
6 & 53 & 20.90\end{array}$ & $\begin{array}{l}-0.04 \\
+0.06 \\
-0.04 \\
-0.04 \\
-0.05\end{array}$ & $\begin{array}{l}\cdots \\
\cdots \\
\cdots \\
\cdots\end{array}$ & $\begin{array}{l}\cdots \\
\cdots \\
\cdots \\
\cdots \\
\cdots\end{array}$ & $\begin{array}{l}\text { c. } \\
\text { c. } \\
\text { c. } \\
\text { c. } \\
\text { c. }\end{array}$ & $\begin{array}{rrr}+21 & 57 & 51.8 \\
+22 & 0 & 5.8 \\
+22 & 1 & 48.6 \\
+22 & 310.5 \\
+22 & 3 & 17.5\end{array}$ & $\begin{array}{l}+1.0 \\
+0.7 \\
+0.6 \\
+0.6 \\
+0.1\end{array}$ & $\begin{array}{l}\cdots \\
\cdots \\
\cdots \\
\cdots \\
\cdots\end{array}$ & $\begin{array}{l}\cdots \\
\cdots \\
\cdots \\
\cdots\end{array}$ \\
\hline $\begin{array}{r}21.4 \\
26.4 \\
28.3 \\
\text { Dec. } 7.6 \\
8.6\end{array}$ & $\begin{array}{l}\text { HI. } \\
\text { P. } \\
\text { Hi. } \\
\text { P. } \\
\text { L. }\end{array}$ & $\stackrel{\mathrm{E} .}{\mathrm{W}}$ & $\begin{array}{l}\text { C. } \\
\text { C. } \\
\text { C. } \\
\text { C. } \\
\text { C. }\end{array}$ & $\begin{array}{rrr}6 & 53 & 16.64 \\
6 & 52 & 57.42 \\
6 & 52 & 50.61 \\
7 & 11 & 10.07 \\
7 & 11 & 4.08\end{array}$ & $\begin{array}{l}-0.09 \\
-0.10 \\
-0.09 \\
-0.06 \\
-0.04\end{array}$ & $\begin{array}{l}\cdots \cdots \\
\cdots \\
\cdots \\
\cdots \\
\cdots\end{array}$ & $\begin{array}{l}\cdots \\
\cdots \\
\cdots \\
\cdots \\
\cdots \\
\cdots\end{array}$ & $\begin{array}{l}\text { c. } \\
\text { c. } \\
\text { c. } \\
\text { c. }\end{array}$ & 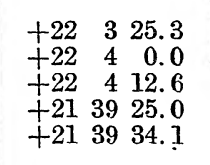 & $\begin{array}{l}+0.5 \\
+0.6 \\
+0.4 \\
+1.1 \\
+0.1\end{array}$ & $\begin{array}{l}\cdots \\
\cdots \\
\cdots \\
\cdots \\
\cdots\end{array}$ & $\begin{array}{l}\cdots \\
\cdots \\
\cdots \\
\cdots\end{array}$ \\
\hline $\begin{array}{r}20.6 \\
27.5 \\
28.5 \\
29.5 \\
1909\end{array}$ & $\begin{array}{l}\text { M. } \\
\text { M. } \\
\text { P. }\end{array}$ & & $\begin{array}{l}\text { c. } \\
\text { c. } \\
\text { c. }\end{array}$ & $\begin{array}{lll}7 & 9 & 46.31 \\
7 & 8 & 57.43 \\
7 & 8 & 50.44 \\
7 & 8 & 43.25\end{array}$ & $\begin{array}{l}-0.10 \\
-0.11 \\
+0.01 \\
-0.04\end{array}$ & $\begin{array}{l}\cdots \\
\cdots \\
\cdots\end{array}$ & $\begin{array}{l}\cdots \\
\cdots \\
\cdots\end{array}$ & $\begin{array}{l}\text { c. } \\
\text { c. } \\
\text { c. }\end{array}$ & $\begin{array}{r}+214145.2 \\
+2143 \quad 7.7 \\
+21 \\
+21 \\
+2133.8\end{array}$ & $\begin{array}{l}+0.2 \\
-0.2 \\
+0.8 \\
+0.9\end{array}$ & $\begin{array}{l}\cdots \\
\cdots \\
\cdots \\
\cdots\end{array}$ & $\begin{array}{l}\cdots \\
\cdots \\
\cdots\end{array}$ \\
\hline $\begin{array}{r}\text { Jan. } \\
1.5 \\
3.5 \\
6.5 \\
18.5 \\
20.5\end{array}$ & $\begin{array}{l}\text { P. } \\
\text { M. } \\
\text { M. } \\
\text { L. }\end{array}$ & W. & $\begin{array}{l}\text { c. } \\
\text { c. } \\
\text { c. } \\
\text { c. } \\
\text { c. }\end{array}$ & $\begin{array}{rrr}7 & 8 & 21.55 \\
7 & 8 & 7.28 \\
7 & 7 & 45.40 \\
7 & 6 & 19.54 \\
7 & 6 & 5.64\end{array}$ & $\begin{array}{l}-0.19 \\
-0.01 \\
-0.19 \\
-0.12 \\
-0.06\end{array}$ & $\begin{array}{l}\cdots \\
\cdots \\
\cdots \\
\cdots \\
\cdots\end{array}$ & $\begin{array}{l}\cdots \\
\cdots \\
\cdots \\
\cdots \\
\cdots\end{array}$ & $\begin{array}{l}\text { c. } \\
\text { c. } \\
\text { c. } \\
\text { c. }\end{array}$ & 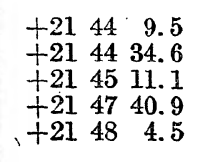 & $\begin{array}{l}+0.3 \\
+0.5 \\
+0.2 \\
+1.2 \\
+0.5\end{array}$ & $\begin{array}{l}\cdots \\
\cdots \\
\cdots \\
\cdots \\
\cdots\end{array}$ & $\begin{array}{l}\cdots \\
\cdots \\
\cdots \\
\cdots\end{array}$ \\
\hline
\end{tabular}




\begin{tabular}{|c|c|c|c|c|c|c|c|c|c|c|c|c|}
\hline \multicolumn{13}{|c|}{ NEPTUNE. } \\
\hline \multicolumn{13}{|c|}{ [No correction for personal equation, see pages'A cLxir and A cLxv, nor for equinox, see page A cLuv, has been applied.] } \\
\hline Date. & 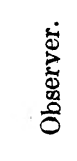 & 宊 & 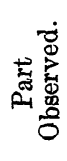 & $\begin{array}{l}\text { Apparent Right } \\
\text { Ascension of } \\
\text { Center. }\end{array}$ & $\begin{array}{c}\text { Correc- } \\
\text { tion to } \\
\text { Am. Eph. }\end{array}$ & $\begin{array}{c}\text { Sidereal } \\
\text { Time of } \\
\text { Transit } \\
\text { of Semi- } \\
\text { diameter. }\end{array}$ & $\begin{array}{c}\text { Correc- } \\
\text { tion to } \\
\text { Am. Eph. }\end{array}$ & 尊 & $\begin{array}{c}\text { Apparent } \\
\text { Declination } \\
\text { of Center. }\end{array}$ & $\begin{array}{c}\text { Correc- } \\
\text { tion to } \\
\text { Am. Eph. }\end{array}$ & $\begin{array}{c}\text { Vertical } \\
\text { Semi- } \\
\text { diameter. }\end{array}$ & $\begin{array}{c} \\
\text { Correc- } \\
\text { tion to } \\
\text { Am. Eph. }\end{array}$ \\
\hline \begin{tabular}{cr}
\multicolumn{2}{c}{1909} \\
Jan. 21.5 \\
22.5 \\
26.4 \\
31.4 \\
Feb. 1.4
\end{tabular} & $\begin{array}{l}\text { M. } \\
\text { P. } \\
\text { L. } \\
\text { P. }\end{array}$ & $\mathrm{W}$. & $\begin{array}{l}\text { C. } \\
\text { C. } \\
\text { C. } \\
\text { C. } \\
\text { C. }\end{array}$ & $\begin{array}{ccc}\mathrm{h} & -\mathrm{m} & \mathrm{s} \\
7 & 5 & 58.71 \\
7 & 5 & 51.84 \\
7 & 5 & 24.82 \\
7 & 4 & 52.50 \\
7 & 4 & 46.15\end{array}$ & $\begin{array}{l}-0.07 \\
-0.06 \\
-0.11 \\
-0.05 \\
-0.13\end{array}$ & $\begin{array}{l}\mathrm{s} \\
\cdots \\
\cdots \\
\cdots \\
\cdots \\
\cdots\end{array}$ & $\begin{array}{l}\mathrm{s} \\
\ldots . \\
\ldots . \\
\ldots . \\
\ldots .\end{array}$ & $\begin{array}{l}\text { c. } \\
\text { c. } \\
\text { c. } \\
\text { c. }\end{array}$ & 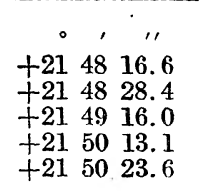 & $\begin{array}{r}11 \\
+0.5 \\
+0.3 \\
+0.7 \\
+0.5 \\
-0.2\end{array}$ & $\begin{array}{l}\text { " } \\
\cdots \\
\cdots \\
\cdots \\
\cdots \\
\cdots\end{array}$ & $\begin{array}{l}\prime \prime \\
\ldots \\
\cdots \\
\cdots \\
\cdots\end{array}$ \\
\hline 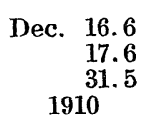 & $\begin{array}{l}\text { M. } \\
\text { L. } \\
\text { P. }\end{array}$ & E. & $\begin{array}{l}\text { c. } \\
\text { C. }\end{array}$ & $\begin{array}{rrr}7 & 20 & 2.24 \\
7 & 19 & 55.70 \\
7 & 18 & 19.76\end{array}$ & $\begin{array}{l}-0.02 \\
-0.08 \\
-0.09\end{array}$ & $\begin{array}{l}\ldots \\
\cdots \\
\cdots\end{array}$ & $\begin{array}{l}\ldots . \\
\cdots \\
\cdots\end{array}$ & $\begin{array}{l}\text { c. } \\
\text { c. } \\
\text { c. }\end{array}$ & $\begin{array}{l}+212715.6 \\
+212729.7 \\
+213034.0\end{array}$ & $\begin{array}{r}0.0 \\
+1.7 \\
+0.3\end{array}$ & $\begin{array}{l}\cdots \\
\cdots \\
\cdots\end{array}$ & $\begin{array}{l}\cdots \\
\cdots \\
\cdots\end{array}$ \\
\hline $\begin{array}{r}\text { Jan. } \\
3.5 \\
7.5 \\
8.5 \\
10.5 \\
15.5\end{array}$ & $\begin{array}{l}\text { P. } \\
\text { P. } \\
\text { L. } \\
\text { M. } \\
\text { L. }\end{array}$ & & $\begin{array}{l}\text { C. } \\
\text { C. } \\
\text { C. } \\
\text { C. } \\
\text { C. }\end{array}$ & $\begin{array}{lrr}7 & 17 & 58.29 \\
7 & 17 & 29.56 \\
7 & 17 & 22.31 \\
7 & 17 & 7.84 \\
7 & 16 & 31.94\end{array}$ & $\begin{array}{l}-0.12 \\
-0.05 \\
-0.09 \\
-0.13 \\
-0.13\end{array}$ & $\begin{array}{l}\cdots \\
\cdots \\
\cdots\end{array}$ & $\begin{array}{l}\ldots . . \\
\ldots . \\
\cdots \\
\cdots\end{array}$ & $\begin{array}{l}\text { c. } \\
\text { c. } \\
\text { c. } \\
\text { c. } \\
\text {. }\end{array}$ & 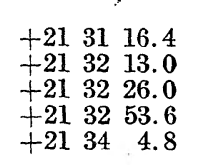 & $\begin{array}{l}+1.1 \\
+1.9 \\
+0.9 \\
+0.3 \\
+1.4\end{array}$ & $\begin{array}{l}\ldots . \\
\cdots \\
\cdots \\
\cdots \\
\cdots\end{array}$ & $\begin{array}{l}\cdots \\
\cdots \\
\cdots \\
\cdots\end{array}$ \\
\hline $\begin{array}{l}16.5 \\
19.5 \\
25.5 \\
26.4 \\
29.4\end{array}$ & $\begin{array}{l}\text { P. } \\
\text { L. } \\
\text { P. } \\
\text { L. }\end{array}$ & & $\begin{array}{l}\text { C. } \\
\text { C. } \\
\text { C. } \\
\text { C. } \\
\text { C. }\end{array}$ & $\begin{array}{rrr}7 & 16 & 24.87 \\
7 & 16 & 3.59 \\
7 & 15 & 22.13 \\
7 & 15 & 15.24 \\
7 & 1.4 & 55.40\end{array}$ & $\begin{array}{l}-0.07 \\
-0.11 \\
-0.01 \\
-0.13 \\
+0.01\end{array}$ & $\begin{array}{l}\cdots \\
\cdots \\
\cdots \\
\cdots \\
\cdots\end{array}$ & $\begin{array}{l}\ldots . . \\
\ldots . \\
\cdots \\
\ldots\end{array}$ & $\begin{array}{l}\text { c. } \\
\text { c. } \\
\text { c. } \\
\text { c. }\end{array}$ & $\begin{array}{r}+213418.4 \\
+213459.6 \\
+213621.5 \\
+213634.6 \\
+213714.4\end{array}$ & $\begin{array}{l}+1.0 \\
+0.6 \\
+0.7 \\
+0.4 \\
+0.7\end{array}$ & $\begin{array}{l}\cdots \\
\cdots \\
\cdots \\
\cdots \\
\cdots\end{array}$ & $\begin{array}{l}\cdots \\
\cdots \\
\cdots \\
\cdots\end{array}$ \\
\hline 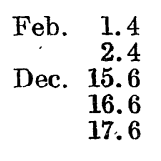 & $\begin{array}{l}\mathrm{M} . \\
\mathrm{P} . \\
\mathrm{M} . \\
\mathrm{L} .\end{array}$ & & $\begin{array}{l}\text { C. } \\
\text { C. } \\
\text { C. } \\
\text { C. }\end{array}$ & $\begin{array}{lll}7 & 14 & 35.86 \\
7 & 14 & 29.53 \\
7 & 29 & 54.23 \\
7 & 29 & 48.15 \\
7 & 29 & 41.83\end{array}$ & $\begin{array}{l}-0.08 \\
-0.06 \\
-0.15 \\
-0.02 \\
-0.05\end{array}$ & $\begin{array}{l}\cdots \\
\cdots \\
\cdots \\
\cdots\end{array}$ & $\begin{array}{l}\ldots . . \\
\cdots . \\
\cdots . \\
\cdots\end{array}$ & $\begin{array}{l}\text { c. } \\
\text { c. } \\
\text { c. } \\
\text { c. } \\
\text { c. }\end{array}$ & $\begin{array}{rrr}+21 & 37 & 52.8 \\
+21 & 38 & 5.7 \\
+21 & 11 & 11.1 \\
+21.11 & 25.6 \\
+21 & 11 & 38.2\end{array}$ & $\begin{array}{r}+0.5 \\
+0.7 \\
+0.3 \\
+0.9 \\
0.0\end{array}$ & $\begin{array}{l}\cdots \\
\cdots \\
\cdots \\
\cdots \\
\cdots\end{array}$ & $\begin{array}{l}\cdots \\
\cdots \\
\cdots \\
\cdots\end{array}$ \\
\hline $\begin{array}{r}20.6 \\
26.6 \\
1911\end{array}$ & P. & & C. & $\begin{array}{l}72922.56 \\
7 \quad 28 \quad 42.54\end{array}$ & $\begin{array}{l}-0.08 \\
-0.01\end{array}$ & .. & $\ldots$ & C. & $\begin{array}{l}+211220.8 \\
+211347.3\end{array}$ & $\begin{array}{l}+0.9 \\
+1.0\end{array}$ & $\cdots$ & $\cdots$ \\
\hline $\begin{array}{ll}\text { Jan. } \quad 4.5 \\
\quad 9.5\end{array}$ & L. & & C. & $\begin{array}{l}72739.50 \\
7273.71\end{array}$ & $\begin{array}{l}-0.04 \\
-0.08\end{array}$ & $\ldots$ & $\ldots$. & C. & $\begin{array}{rrr}+21 & 16 & 3.8 \\
+21 & 17 & 20.8\end{array}$ & $\begin{array}{l}+0.8 \\
+0.3\end{array}$ & $\cdots$ & $\cdots$ \\
\hline $\begin{array}{l}10.5 \\
15.5 \\
16.5 \\
23.5 \\
24.5\end{array}$ & $\begin{array}{l}\mathrm{P} . \\
\mathrm{M} . \\
\mathrm{P} . \\
\mathrm{M} . \\
\mathrm{P} .\end{array}$ & & $\begin{array}{l}\text { C. } \\
\text { C. } \\
\text { C. } \\
\text { C. }\end{array}$ & $\begin{array}{lll}7 & 26 & 56.56 \\
7 & 26 & 20.73 \\
7 & 26 & 13.57 \\
7 & 25 & 24.15 \\
7 & 25 & 17.22\end{array}$ & $\begin{array}{l}-0.06 \\
-0.04 \\
-0.05 \\
-0.06 \\
-0.05\end{array}$ & $\begin{array}{l}\cdots \\
\cdots . \\
\cdots \\
\cdots \\
\cdots\end{array}$ & $\begin{array}{l}\ldots . . \\
\ldots . \\
\ldots . \\
\ldots\end{array}$ & $\begin{array}{l}\text { c. } \\
\text { c. } \\
\text { c. } \\
\text { c. }\end{array}$ & 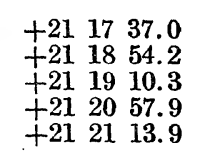 & $\begin{array}{l}+0.9 \\
+0.1 \\
+0.6 \\
+0.2 \\
+1.0\end{array}$ & $\begin{array}{l}\cdots \\
\cdots \\
\cdots \\
\cdots \\
\cdots\end{array}$ & $\begin{array}{l}\ldots \\
\ldots \\
\cdots \\
\cdots\end{array}$ \\
\hline 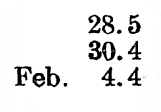 & $\begin{array}{l}\mathrm{L} . \\
\mathrm{M} .\end{array}$ & $\mathrm{E}_{\mathbf{1}}$ & $\begin{array}{l}\text { c. } \\
\text { c. }\end{array}$ & $\begin{array}{llr}7 & 24 & 49.85 \\
7 & 24 & 36.49 \\
7 & 24 & 4.02\end{array}$ & $\begin{array}{l}-0.08 \\
-0.05 \\
-0.11\end{array}$ & $\begin{array}{l}\cdots \\
\cdots \\
\cdots\end{array}$ & $\ldots$ & $\begin{array}{l}\text { c. } \\
\text { c. }\end{array}$ & 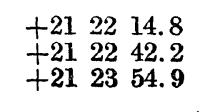 & $\begin{array}{r}+2.1 \\
0.0 \\
+1.1\end{array}$ & $\begin{array}{l}\cdots \\
\cdots \\
\cdots\end{array}$ & $\begin{array}{l}\cdots \\
\cdots\end{array}$ \\
\hline
\end{tabular}

